PNNL-24382, Rev 1.0 WTP-RPT-239, Rev. 1.0

Pacific Northwest

\title{
Implications of Upwells as Hydrodynamic Jets in a Pulse Jet Mixed System
}

\section{February 2017}

LF Pease

JA Bamberger
MJ Minette 


\title{
DISCLAIMER
}

This report was prepared as an account of work sponsored by an agency of the United States Government. Neither the United States Government nor any agency thereof, nor Battelle Memorial Institute, nor any of their employees, makes any warranty, express or implied, or assumes any legal liability or responsibility for the accuracy, completeness, or usefulness of any information, apparatus, product, or process disclosed for any uses other than those related to WTP for DOE, or represents that its use would not infringe privately owned rights. Reference herein to any specific commercial product, process, or service by trade name, trademark, manufacturer, or otherwise does not necessarily constitute or imply its endorsement, recommendation, or favoring by the United States Government or any agency thereof, or Battelle Memorial Institute. The views and opinions of authors expressed herein do not necessarily state or reflect those of the United States Government or any agency thereof.

\author{
PACIFIC NORTHWEST NATIONAL LABORATORY \\ operated by \\ BATTELLE for the \\ UNITED STATES DEPARTMENT OF ENERGY \\ under Contract DE-AC05-76RL01830
}

Printed in the United States of America
Available to DOE and DOE contractors from the Office of Scientific and Technical Information,
P.O. Box 62, Oak Ridge, TN 37831-0062;
ph: (865) 576-8401
fax: (865) 576-5728
email: reports@adonis.osti.gov

Available to the public from the National Technical Information Service

5301 Shawnee Rd., Alexandria, VA 22312 ph: (800) 553-NTIS (6847)

email: orders@ntis.gov <http://www.ntis.gov/about/form.aspx> 
PNNL-24382, Rev 1.0

WTP-RPT-239, Rev. 1.0

\title{
Implications of Upwells as Hydrodynamic Jets in a Pulse Jet Mixed System
}

\author{
LF Pease \\ MJ Minette \\ JA Bamberger
}

February 2017

Test Specification: N/A

Work Authorization: WA\# 048

Test Plan:

TP-WTPSP-132, Rev 0.0

Test Exceptions:

N/A

Focus Area:

Pretreatment

Test Scoping Statement(s):

QA Technology Level:

Project Number:

NA

Basic Research

66560

Prepared for

the U.S. Department of Energy

under Contract DE-AC05-76RL01830

Pacific Northwest National Laboratory

Richland, Washington 99352 



\section{Revision History}

\begin{tabular}{|c|c|c|c|}
\hline $\begin{array}{c}\text { Revision } \\
\text { Number }\end{array}$ & $\begin{array}{c}\text { Interim } \\
\text { Change No. } \\
\end{array}$ & Effective Date & Description of Change \\
\hline 0 & 0 & $8 / 27 / 2015$ & Initial issue. \\
\hline 1 & 0 & $2 / 3 / 2017$ & $\begin{array}{l}\text { Resolution of NCR OTS-03616 Rev. } 2 \text { produced small } \\
\text { changes for the four-part simulant, which affects Tables } \\
2.4,3.3 \text {, and } 7.2 \text {, Figures } 2.13 \mathrm{e}, 2.14 \mathrm{ab}, 3.1,7.3 \mathrm{e} \text {, and } 7.4, \\
\text { and one text entry (p2.27). } \\
\text { W replaces } \mathrm{Al}_{2} \mathrm{O}_{3} \text { in multipart simulant composition in Tables } \\
2.2,2.4,3.2,3.3 \text {, and } 7.2 \text {. } \\
\text { Report conclusions were not affected. }\end{array}$ \\
\hline
\end{tabular}




\section{Executive Summary}

This report evaluates the physics of the upwell flow in pulse jet mixed systems in the Hanford Tank Waste Treatment and Immobilization Plant (WTP). Although the initial downward flow and radial flow from jets characteristic of pulse jet mixers (PJMs) has been analyzed, the upwells have received considerably less attention despite having significant implications for vessel mixing. Do the upwells behave like jets? How do the upwells scale? When will the central upwell break through? What proportion of the vessel is blended by the upwells themselves? Indeed, how the physics of the central upwell is affected by multiple PJMs (e.g., six in the proposed mixing vessels), non-Newtonian rheology, and significant multicomponent solids loadings remain unexplored.

The central upwell must satisfy several criteria to be considered a free jet. First, it must travel for several diameters in a nearly constant direction. Second, its velocity must decay with the inverse of elevation. Third, it should have an approximately Gaussian profile. Fourth, the influence of surface and body forces must be negligible. A combination of historical data in a $12.75 \mathrm{ft}$ test vessel (Bontha et al. 2003a, b; Bamberger et al. 2005), newly analyzed data from the $8 \mathrm{ft}$ test vessel, and conservation of momentum arguments derived specifically for PJM operating conditions demonstrate that the central upwell satisfies these criteria where vigorous surface flow is achieved.

An essential feature of scaling from one vessel to the next is the requirement that the underlying physics does not change adversely. One may have confidence in scaling if 1) correlations and formulas capture the relevant physics; 2) the underlying physics does not change from the conditions under which it was developed to the conditions of interest; 3) all factors relevant to scaling have been incorporated, including flow, material, and geometric considerations; and 4) the uncertainty in the relationships is sufficiently narrow to meet required specifications. Although the central upwell satisfies these criteria when vigorous surface flow is achieved, not all available data follow the free jet profile for the central upwell, particularly at lower nozzle velocities. Alternative flow regimes are considered, and new models for cloud height, "cavern" height, and the rate of jet penetration (jet celerity) are benchmarked against data to anchor scaling analyses.

This analytical modeling effort has significant implications for vessel mixing, because jet physics underlies "cavern" height, cloud height, and the volume of mixing considerations. A new four-parameter cloud height model compares favorably to experimental results where input parameters are available. This model is predictive of surface flow in $8 \mathrm{ft}$ vessel tests with the two-part simulant. Analysis of the upwell in the presence of yield stresses finds evidence of expanding turbulent jets, confined turbulent jets, and confined laminar flows. For each, the critical elevation at which jet momentum depletes is predicted, which compares favorably to experimental "cavern" height data. Partially coupled momentum and energy balances suggest that these are limiting cases of a gradual transition from a turbulent expanding flow to a confined laminar flow. This analysis of the central upwell alone lays essential groundwork for complete analysis of mode three mixing (i.e., breakthrough ${ }^{1}$ with slow peripheral mixing). Consideration

\footnotetext{
${ }^{1}$ Breakthrough is colloquially used to describe jet flow driving surface flow for both Newtonian and non-Newtonian simulants. However, Bontha et al. (2015) reserves the term breakthrough for yield stress materials. Here the term surface flow refers to both breakthrough in yield stress materials and jet induced surface flow in Newtonian simulants. The terms breakthrough region to denote the region of the fluid surface disturbed by the central upwell and breakthrough velocity to denote the velocity that the central upwell would have as projected to the fluid surface have both been retained.
} 
of jet celerity shows that the rate of jet penetration is a governing consideration in surface flow.

Estimates of the volume of mixing are presented.

The evaluation of the physics related to the central upwell in a pulse jet mixed vessel has identified the following:

- The central upwell behaves as a free jet in the absence of body and surface forces as supported by consideration of suitable test data.

- When the axial velocity of this free jet is scaled on the jet nozzle velocity and the elevation on the nozzle diameter, the data collapse onto curves that depend only on the jet coefficient and the location of a virtual origin with respect to the vessel bottom. These parameters have been extracted from historical and $8 \mathrm{ft}$ testing with multiple simulants at multiple scales.

- The radial distribution of velocities is essential to quantify flow within mixing vessels. The fractional width of the jet was approximately $10 \%$ to $20 \%$ of the vessel width in the $12.75 \mathrm{ft}$ vessel. Available data leave uncertainty in the radial spread of the jet of approximately a factor of three.

- The Poirier-Etchells criterion ${ }^{1}$ presents a cutoff velocity for jet turbulence that is approximately predictive of surface flow when coupled with the free jet equation.

- Conservation of momentum may be used to predict jet coefficients for free jets. A geometric approximation may be used to estimate how the central upwell depends on the number of PJMs within a mixing vessel. Shear forces along the vessel bottom only weakly influence the upwell flows. Predicted jet coefficients for a free jet typically remain within a factor of three of measured jet coefficients for Newtonian simulants.

- A general framework that combines momentum and species conservation establishes relationships among jet momentum, buoyancy, and yield stresses. The framework is sufficiently general to permit simultaneous evaluation of buoyancy and yield stresses (e.g., for simulants with low yield stresses and density stratification).

- Jet flow governs the central upwell with and without solids until the negative buoyancy force becomes influential. The Froude number based on nozzle diameter without density contributions collapses the data from $8 \mathrm{ft}$ vessel tests regardless of pulse volume fraction, PJM firing sequence, or Newtonian simulant with separate critical Froude numbers for each nozzle diameter. A Froude number based on tank diameter would not lead to a single critical Froude number. The Froude number alone in its various forms, therefore, cannot reliably predict scale-up of flows below the upper surface because the critical Froude number varies with at least nozzle diameter.

- A new cloud height model was developed from first principles and validated against the $8 \mathrm{ft}$ vessel tests with the two-part simulant. This model requires four parameters: the Froude number, the jet coefficient, the ratio of the density at the base of the upwell to the density far from the jet centerline, and the ratio of the depth of the virtual origin below the vessel bottom to the nozzle diameter. The first is clearly scale dependent, and the second is scale independent. How the latter two scale between

\footnotetext{
${ }^{1}$ This criterion asserts that at any location the local velocity must be in excess of a cutoff velocity to remain turbulent. When coupled with the free jet velocity equation that relates the local velocity to elevation (Eq. (2.1)), the elevation at which turbulence will fail, and consequently if the turbulent jet will fail, may be estimated. This elevation may be compared to the elevation of the fluid surface to predict whether the upwell will reach the surface as a metric of surface flow.
} 
vessel sizes remains unclear. When these are known, this cloud height model predicted if surface flow occurred.

- Conservation of energy alone suggests that in a yield stress material, the jet initially expands and then contracts. Without solving the coupled momentum and energy conservation equations together, three limiting cases are considered. First, an expanding turbulent jet predicts the critical elevation at which the velocity ceases due to insufficient jet momentum. This model, validated against "cavern" height data in laponite, collapses the data from multiple scales onto a single curve. Second, the turbulent confined jet of constant radius shows that the jet radius increases as the yield Reynolds number (defined on the Bingham yield stress, not the shear strength) increases until it is large enough that viscous forces become influential. Analysis shows that the upwell flow loses turbulence and becomes laminar. Third, when upwell flow is laminar (supported by measured radial velocity profiles), the square of the ratio of the centerline velocity to the nozzle velocity decays linearly with the elevation scaled on nozzle diameter. The third case represents velocity data in the $8 \mathrm{ft}$ vessel well at $8 \mathrm{~m} / \mathrm{s}$. Further work is needed to consider fully coupled momentum and energy to add fidelity to these relationships.

- Flows within mixing vessels holding yield stress materials may be independent of geometric scaling at constant nozzle velocity when the yield Reynolds number is sufficiently small so long as the virtual origin does not shift between scales. When the ratio of the yield Reynolds number to jet Reynolds number, which represents the ratio of viscous stresses to yield stresses, is order one, the jet Reynolds number becomes influential. For the $16 \mathrm{ft}$ vessel, this ratio is order one. The jet Reynolds number depends on geometric scale, so that scaling between vessels may depend on geometric scale. See Wells et al. (2015) for further analysis.

- Velocities and diameters of the upwell flows in the presence of yield stress materials are presented, laying important groundwork for evaluating the surface and downwell flows that govern mixing/blending outside the upwell flow (i.e., the remainder of mode three mixing). The radial velocity distribution determines the local effective viscosity, which in turn governs hydrogen release.

- The rate at which the upwelling jet propagates or penetrates toward the fluid surface (termed jet celerity) determines whether the jet achieves surface flow or not.

- Simply integrating the centerline jet velocity underestimates the time a free jet takes to rise to the fluid surface. The time-dependent conservation of momentum equation was solved to show that the radial distribution of velocities serves as a key correction factor. Including the radial distributions improves prediction of surface flow. The time for the jet to transit from the vessel seam, tangent, or knuckle to the fluid surface comprises a strong majority of jet transit time.

- Conservation of momentum shows jet flow along the walls to be independent of geometric scaling (the vessel diameter is divided by the nozzle diameter), but the velocity decays with the inverse of the square root of the elevation instead of with the inverse of the elevation directly. Even though more of the PJM momentum flows toward the wall, the momentum spreads out over the perimeter and is countered by a down flow, so that the jet flow along the wall is typically both weaker and slower than the central upwell. Therefore, this report focuses on the central upwell.

- Jet physics permits estimation of the volume mixed (i.e., sheared) by the central upwell during a single pulse at the moment surface flow is achieved (lateral flow not included). Mixing caused by only the central upwell as it achieves surface flow is estimated to vary from $<0.5 \%$ to $\sim 75 \%$ depending on the radial distribution of velocities (Table 9.1). Additional flow may proceed after 
surface flow has been achieved until the end of the PJM pulse, in other parts of the vessel (below seam, $18 \%$; wall upwell, typically $<15 \%$ ), and due to the leading vortex (a measurement opportunity in the $16 \mathrm{ft}$ vessel with radially distributed velocity probes).

\section{Quality Requirements}

Pacific Northwest National Laboratory (PNNL) complies with the requirements found in the following standards and implements them in their Waste Treatment Plant Support Program (WTPSP) Quality Assurance Program:

- ASME NQA-1-2000, Quality Assurance Requirements for Nuclear Facility Applications, Part I, Requirements for Quality Assurance Programs for Nuclear Facilities

- ASME NQA-1-2000, Part II, Subpart 2.7, Quality Assurance Requirements for Computer Software for Nuclear Facility Applications

- ASME NQA-1-2000, Part IV, Subpart 4.2, Guidance on Graded Application of Quality Assurance (QA) Requirements for Nuclear-Related Research and Development.

Records will be stored as hardcopy records in a 2-hour fire-rated container.

This project recognizes that quality assurance applies in varying degrees to a broad spectrum of research and development (R\&D) in the technology life cycle. The R\&D elements in the technology life cycle are listed below. The WTPSP uses a graded approach for the application of the quality assurance controls such that the level of analysis, extent of documentation, and degree of rigor of process control are applied commensurate with their significance, importance to safety, life cycle state of work, or programmatic mission. The technology life cycle is characterized by flexible and informal quality assurance activities in basic research, which becomes more structured and formalized through the applied R\&D stages.

- Basic Research: Basic Research consists of research tasks that are conducted to acquire and disseminate new scientific knowledge. During Basic Research, maximum flexibility is desired in order to allow the researcher the necessary latitude to conduct the research.

- Applied Research: Applied Research consists of research tasks that acquire data and documentation necessary to ensure satisfactory reproducibility of results. The emphasis during this stage of a research task is on achieving adequate documentation and controls necessary to be able to reproduce results.

- Development Work: Development Work consists of research tasks moving toward technology commercialization. These tasks still require a degree of flexibility and there is still a degree of uncertainty in many cases. The role of quality in Development Work is to make sure that adequate controls to support movement into commercialization exist.

- Research and Development Support Activities: Support activities are those that are conventional and secondary in nature to the advancement of knowledge or development of technology, but allow the primary purpose of the work to be accomplished credibly. An example of a support activity is controlling and maintaining documents and records. The level of quality for these activities is the same as for Development Work. 
This report was conducted at the Basic Research technology level. 


\section{Acknowledgements}

The authors would like to express appreciation to the professionals who contributed meaningfully to the preparation and quality of this document.

Guidance, support, and mentorship: Loni Peurrung and Michael Summers.

Expert review: Lenna Mahoney, Thomas S. Yokuda, Judith Bamberger, Philip Schonewill, Kurt Recknagle, Elizabeth Golovich, Kenneth Johnson, Loni Peurrung, Michael Summers, Joel Peltier, and Behnoush Haghighian.

Providing source information: Loni Peurrung, Korey Peterson, Carolyne Burns, Christopher Chapman, Phillip Keuhlen, and the entire Test Completion Team for providing the data sets analyzed de novo herein.

Editorial and document control support: Matthew Wilburn, Chrissy Charron, and Mona Champion.

Quality reviews and oversight: Kirsten Meier.

Thoughtful and thought provoking conversations: Loni Peurrung, Beric Wells, Lenna Mahoney, William Kuhn, David Rector, Phillip Gauglitz, Carl Enderlin, Thomas S. Yokuda, Judith Bamberger, John Geeting, Jagan Bontha, James Fort, Richard Daniel, Philip Schonewill, Kurt Recknagle, Elizabeth Golovich, Ellen Baer, Sarah Suffield, Scot Rassat, Diana Tran, David Pfund, Gary Josephson, Jaehun Chun, Thomas Michener, Mark Stewart, Joel Peltier, Michael Poirier, and Leolein Moualeu. 



\section{Acronyms and Abbreviations}

$\begin{array}{ll}\text { APEL } & \text { Applied Process Engineering Laboratory } \\ \text { ASME } & \text { American Society of Mechanical Engineers } \\ \text { BNI } & \text { Bechtel National, Inc. } \\ \text { CFD } & \text { computational fluid dynamics } \\ \text { DAS } & \text { data acquisition system } \\ \text { DVF } & \text { drive volume fraction } \\ \text { FIO } & \text { for information only } \\ \text { KB } & \text { kaolin-bentonite } \\ \text { LTTS } & \text { large tank test stand } \\ \text { PJM } & \text { pulse jet mixer } \\ \text { PNNL } & \text { Pacific Northwest National Laboratory } \\ \text { PVF } & \text { pulse volume fraction } \\ \text { R\&D } & \text { research and development } \\ \text { SRNL } & \text { Savannah River National Laboratory } \\ \text { WTP } & \text { Hanford Tank Waste Treatment and Immobilization Plant } \\ \text { WTPSP } & \text { Waste Treatment Plant Support Program }\end{array}$





\section{Symbols}

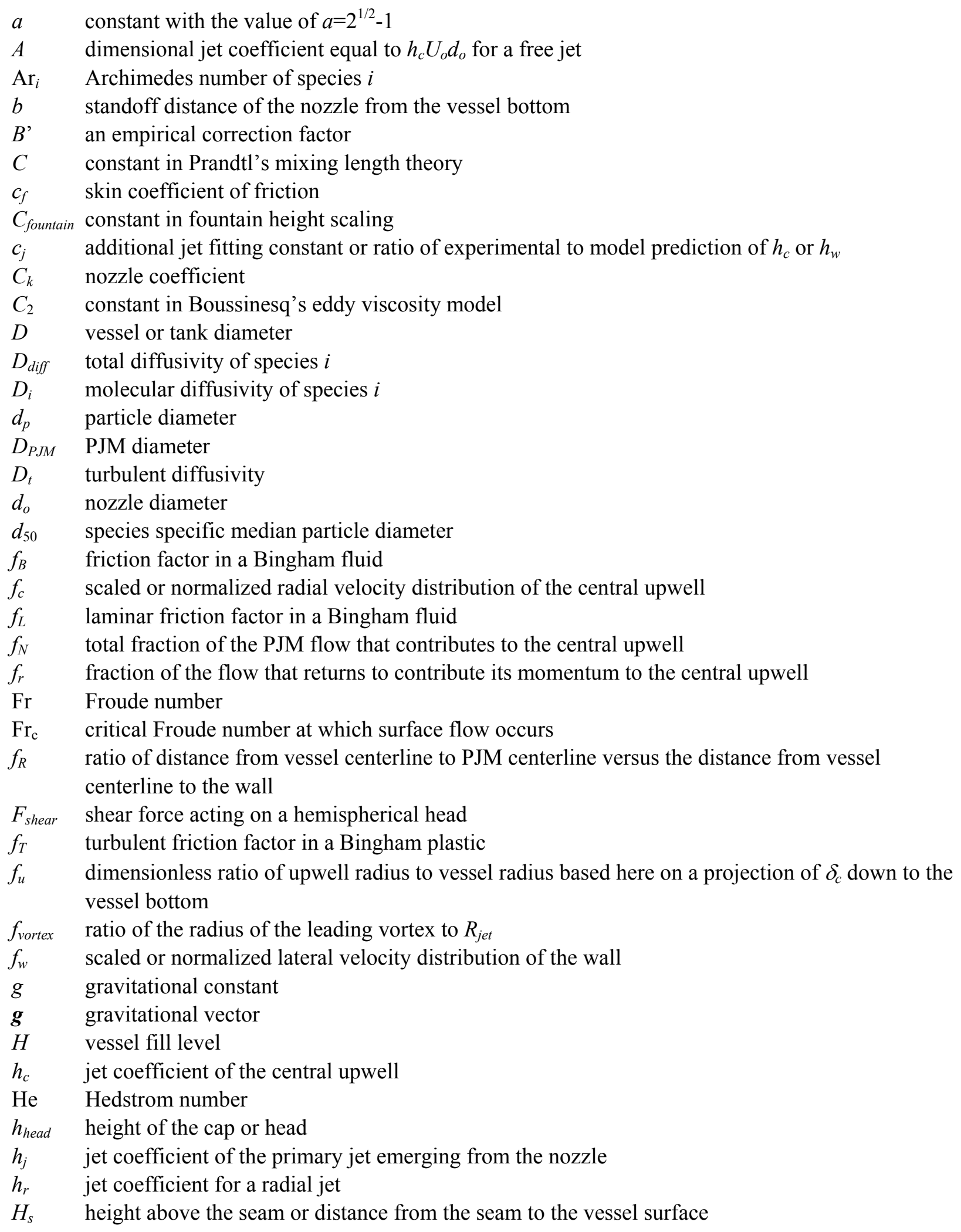




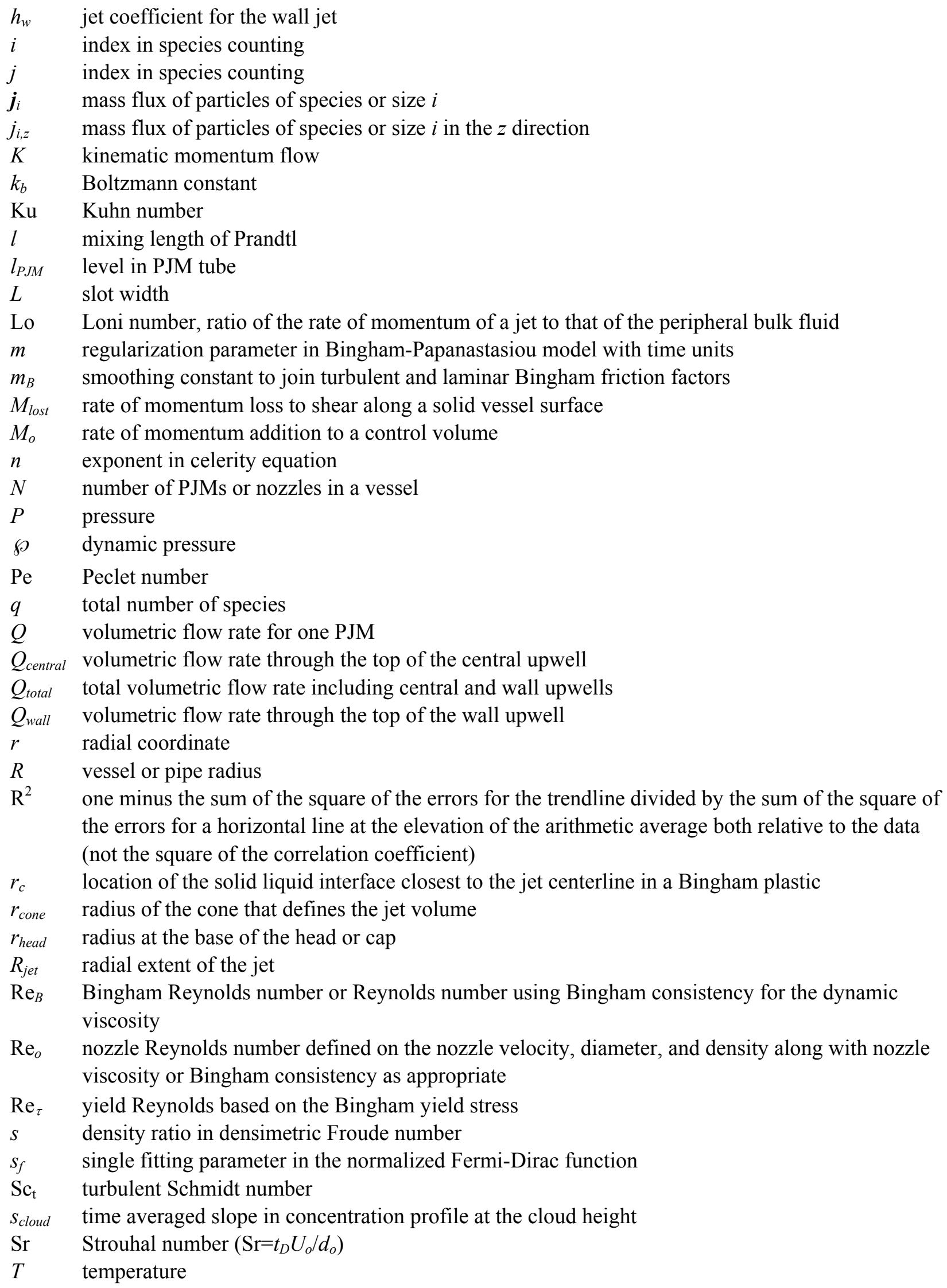




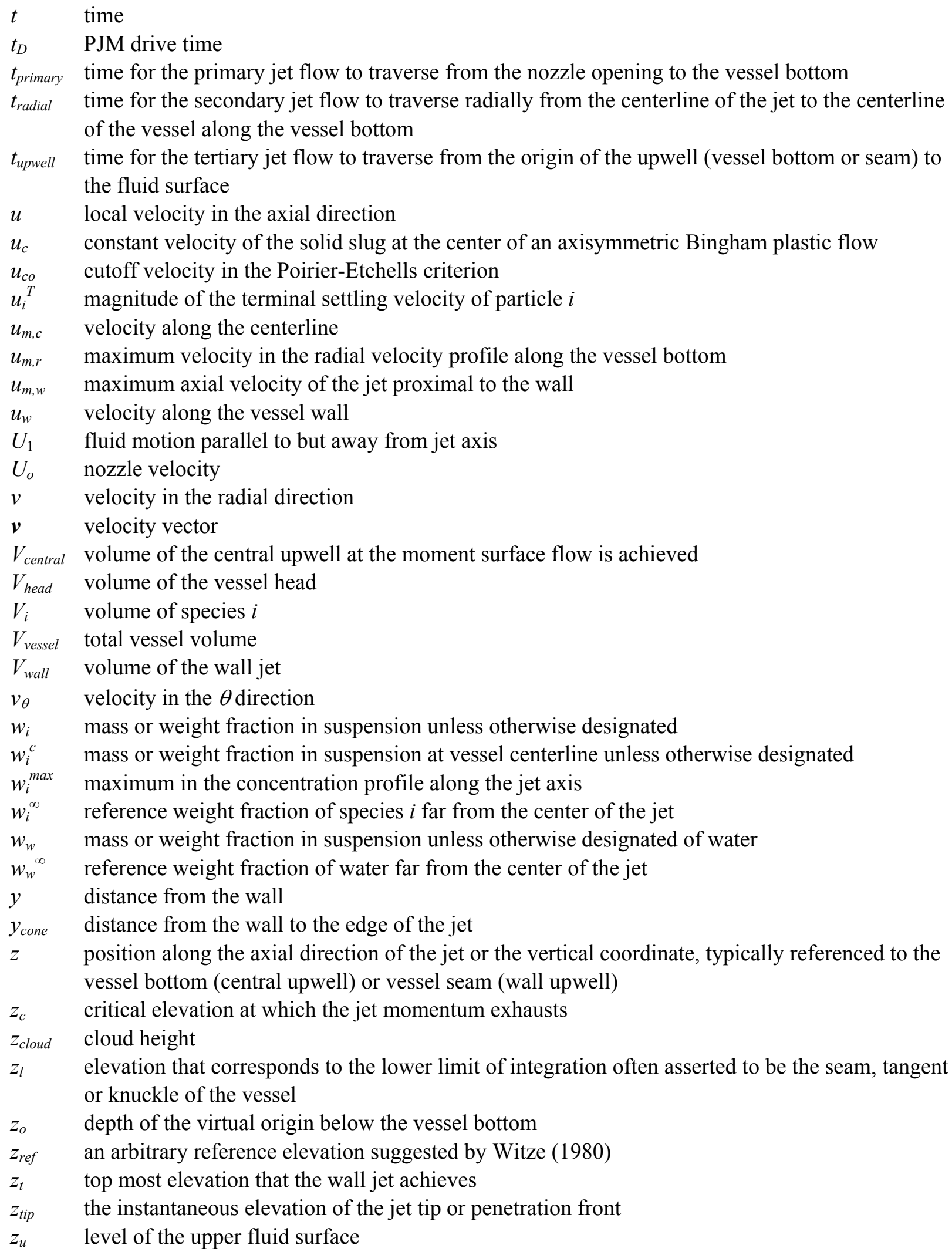





\title{
Greek Symbols
}

\author{
$\alpha \quad$ celerity coefficient \\ $\alpha_{M} \quad$ a Reynolds number based on the Bingham consistency \\ $\alpha_{c}{ }^{\prime} \quad$ a constant \\ $\beta \quad$ constant in determining the Bingham friction factor \\ $\beta_{c} \quad$ jet spread as the ratio of the radius at half the jet velocity to the total axial distance from the \\ virtual origin of a central jet \\ $\beta_{i} \quad$ solutal coefficient of species $i$ \\ $\beta_{w} \quad$ jet spread as the ratio of the radius at half the jet velocity to the total axial distance from the \\ virtual origin of a wall jet \\ $\dot{\gamma} \quad$ shear rate \\ $\delta_{c} \quad$ central jet width defined where the local jet velocity falls to half the jet centerline velocity \\ $\delta_{w} \quad$ wall jet width defined where the local rising jet velocity falls to half the maximum jet velocity \\ (the one farthest from the wall) \\ $\eta_{c} \quad$ dimensionless similarity variable relative to central upwell jet centerline \\ $\eta_{c}{ }^{99}$ dimensionless location where $99 \%$ of the centerline velocity has decayed \\ $\eta_{w} \quad$ dimensionless similarity variable for the rising wall jet \\ $\theta \quad$ angular or azimuthal position \\ $\Theta \quad$ weight fraction ratio \\ $\phi \quad$ angle between the centerline of the primary jet and the vessel bottom \\ $\phi_{i} \quad$ volume fraction of species $i$ \\ $\mu_{0} \quad$ dynamic viscosity or Bingham consistency \\ $\mu_{e f f} \quad$ local effective dynamic viscosity in a Bingham plastic \\ $\mu_{f} \quad$ dynamic viscosity of the fluid surrounding the particle of interest \\ $v \quad$ kinematic viscosity \\ $v_{t} \quad$ turbulent kinematic viscosity \\ $\rho \quad$ local density of the fluid or slurry \\ $\rho_{b} \quad$ mobile slurry density along bottom of vessel (not the density of a particle bed along the vessel \\ bottom) \\ $\rho_{c} \quad$ slurry density along the vessel centerline \\ $\rho_{f} \quad$ density of the fluid (not slurry) surrounding the particle of interest \\ $\rho_{i} \quad$ density of a species $i$, often of the solids \\ $\rho_{\max } \quad$ slurry density at the base of the jet, which is the maximum slurry density associated with the jet \\ $\rho_{o} \quad$ slurry density emerging from the nozzle \\ $\rho_{s, i} \quad$ density of solids $i$ \\ $\rho_{w} \quad$ density of water \\ $\rho_{\infty} \quad$ reference slurry density far from the jet centerline \\ $\tau \quad$ shear stress \\ $\tau \quad$ deviatoric stress tensor \\ $\tau_{\text {lam }} \quad$ shear stress due to non-turbulent viscous stresses \\ $\tau_{\text {lam }}$ deviatoric stress tensor for usual deviatoric stresses \\ $\tau_{\text {turb }} \quad$ shear stress due to turbulent viscous stresses
}


$\tau_{\text {turb }} \quad$ deviatoric stress tensor for the turbulent Reynolds stresses

$\tau_{0} \quad$ Bingham yield stress; shear strength is not used in this report

$\tau_{w} \quad$ shear stress along a wall or solid vessel surface; mathematical expressions for this variable differ by which wall or surface is implied from section to section

$\psi_{\mathrm{B}} \quad$ numerical constant that the represents the integral over the radial distribution of axial velocities in a laminar Bingham plastic flow

$\psi_{\mathrm{i}, \mathrm{j}, \mathrm{k}} \quad$ numerical constant that represent integrals over the radial distributions where $i, j$, and $k$ represent the number of $f_{c}, \eta_{c}$, and $\partial f_{c} / \partial \eta_{c}$ factors, respectively; final factor $k$ optional 


\section{Contents}

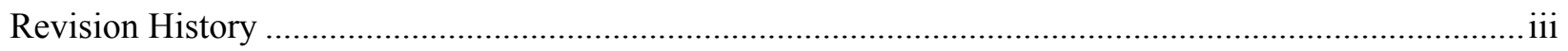

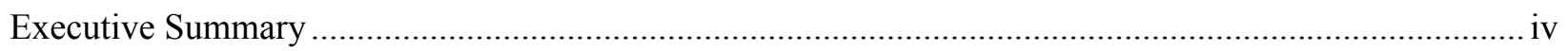

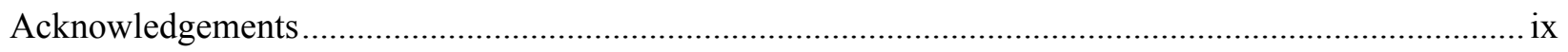

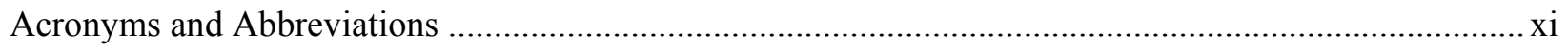

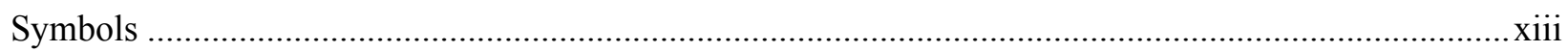

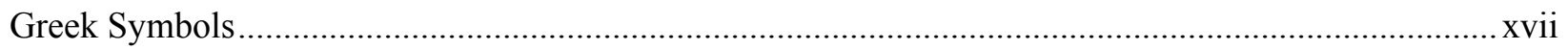

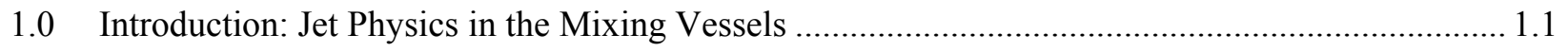

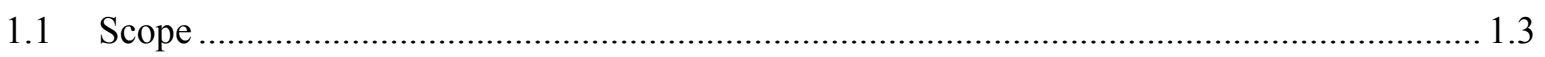

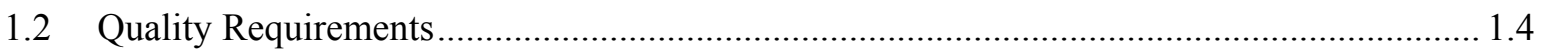

2.0 The Central Upwell is a Free Jet—At Least Sometimes............................................................ 2.1

2.1 Analysis of Historical Centerline Velocity Data ........................................................... 2.1

2.2 Analysis of Historical Radial Velocity Distribution Data ................................................... 2.4

2.3 Attributes of 8-Foot Vessel Testing …........................................................................ 2.11

2.4 Analysis of Centerline Data from the 8 Foot Vessel ........................................................ 2.22

2.5 Minimum Turbulent Velocities and the Poirier-Etchells Criterion ................................... 2.28

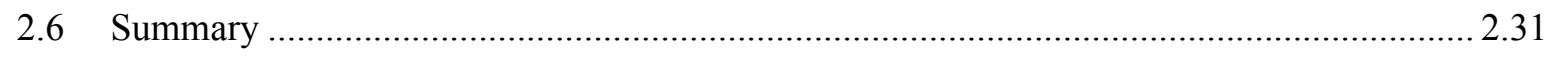

3.0 Upwell Velocity Profile from Momentum Conservation ............................................................ 3.1

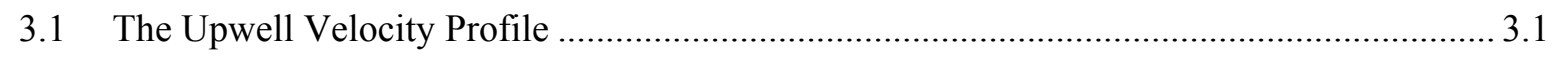

3.2 Upwell Velocity Profile Including Shear Along the Vessel Bottom .................................... 3.4

3.3 Upwell Velocity Profile in the Presence of Bulk Flow ........................................................ 3.7

3.4 Jet Coefficients Compared to Data ................................................................................. 3.11

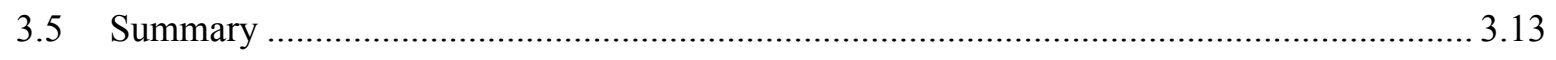

4.0 Framework for Evaluating Alternative Flow Regimes ........................................................... 4.1

4.1 Conservation of Momentum Including Yield and Buoyancy ............................................. 4.1

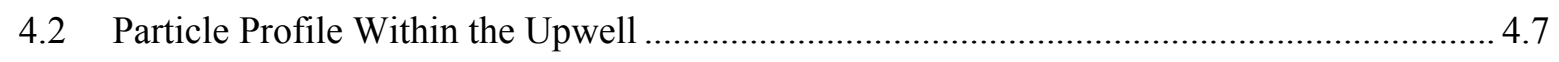

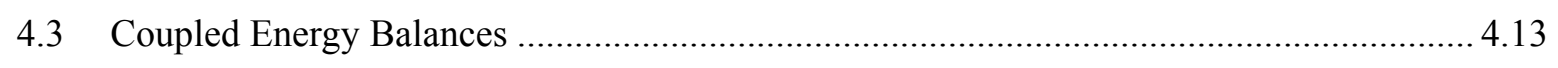

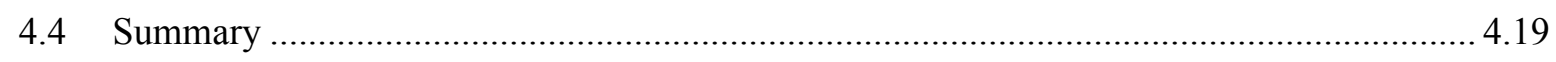

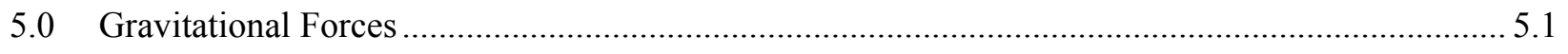

5.1 Negative Buoyancy, the Froude Number, and Fountains ............................................... 5.1

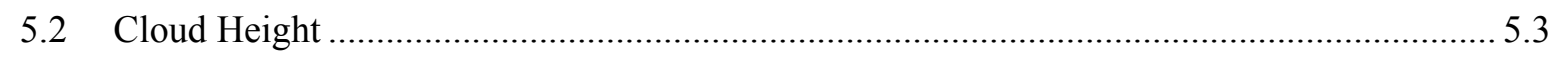

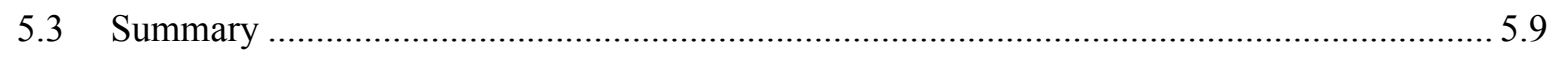

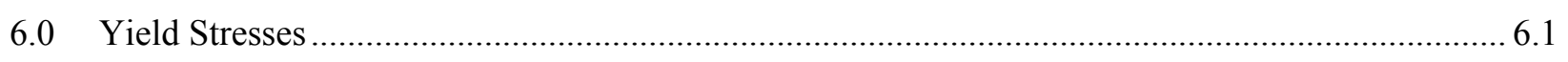

6.1 Turbulent Expanding Jets in Yield Stress Materials ......................................................... 6.1

6.2 Confined Jets of Constant Radius.................................................................................. 6.5

6.3 Bingham Flow in Constant Radius Laminar Tubes ..................................................... 6.11

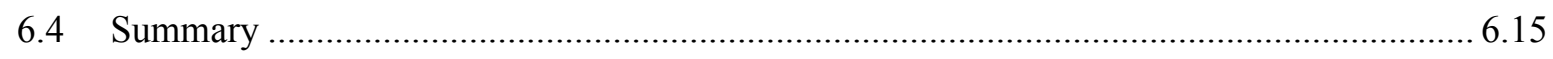




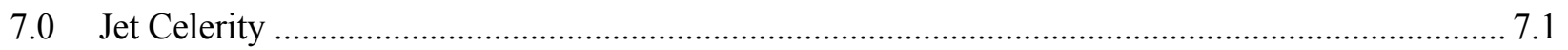

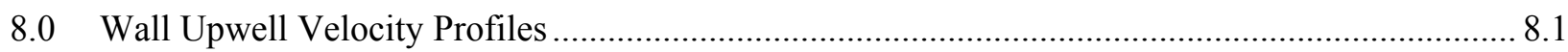

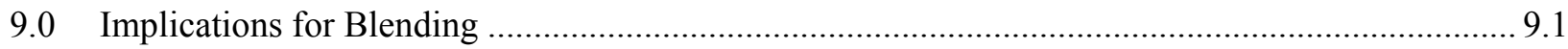

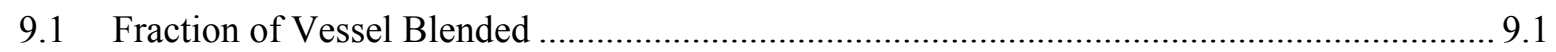

9.2 Why the Jet Cone Alone May Underestimate the Mixing Volume...................................... 9.6

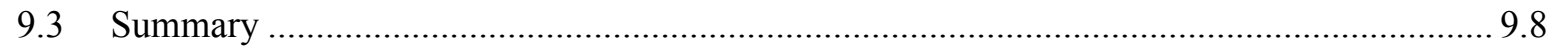

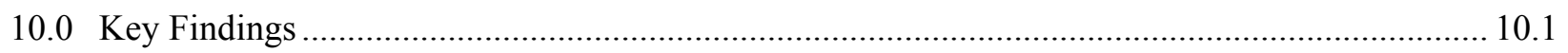

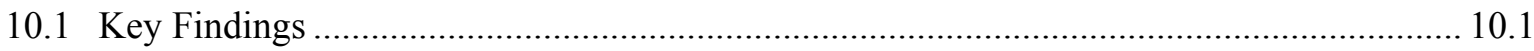

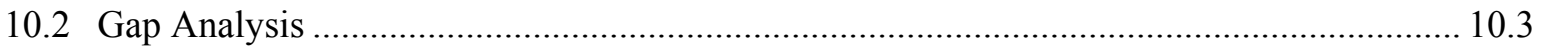

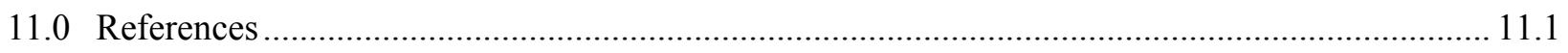

Appendix A Historical Breakthrough Definition and Observations .................................................... A.1 


\section{Figures}

Figure 1.1. Visualization of Flow Within a Pulse Jet Mixed Vessel Between PJMs, Highlighting the Sheared Regions Including the Flow Along the Vessel Bottom and the Central Upwell. This report evaluates the central and wall upwells. Simulation for $16 \mathrm{ft}$ vessel with $U_{o}=12$ $\mathrm{m} / \mathrm{s}, d_{o}=4$ inches, $\mu_{o}=30 \mathrm{mPa} \cdot \mathrm{s}$, and $\rho=1200 \mathrm{~kg} / \mathrm{m}^{3}$ without solids. Asymmetry in central upwell due to suction pipe (not shown) blocking upward momentum. Courtesy of Kurt Recknagle.

Figure 2.1. Compilation of the Historical Central Upwell Velocity Data with Upwell Velocity and Elevation Scaled on a Nozzle Velocity and the Nozzle Diameter, respectively. Curve fits from Eq. (2.1). Vertical line at $z / d_{o}=48$ represents the dimensionless fill height of the proposed standard high solids vessel and the geometrically scaled $8 \mathrm{ft}$ vessel for the 1.94 inch nozzle. The nominal fill height of the 2.82 and $12.75 \mathrm{ft}$ vessels was at $z / d_{o}=29-36$.

Figure 2.2. The Upwell Velocity as a Function of Radial Position in Water in the $12.75 \mathrm{ft}$ Diameter Test Vessel in the LTTS in PNNL 336 Building (Bontha et al. 2003a). PJMs located in the corners outside the field of view. Original caption: "Figure 5.13. Contour Maps of the Maximum Velocities Measured in During the Large Tank Hydrodynamic Tests:

(a) 1.308 m (51.5 in) Horizontal Plane and (b) 1.842 m (72.5 in) Horizontal Plane."

Figure 2.3. The Upwell Velocity as a Function of Radial Position in Water in the $12.75 \mathrm{ft}$

Diameter Test Vessel in the LTTS in PNNL 336 Building (Bontha et al. 2003b) Measured at the Centerline and Vertices of the Bounding Polygon. The pulse tubes are located in the corners outside the field of view. Original captions: (left) "Figure 4.2. Contour Map Showing the Fluid Velocities in a $2 \mathrm{ft}$ Diameter Core Around the Center of the Tank and an Elevation of 30 inches $(0.762 \mathrm{~m})$ " and (right) "Figure 4.3. Contour Map Showing the Fluid Velocities in a $2 \mathrm{ft}$ Diameter Core Around the Center of the Tank and an Elevation of 54 inches (1.3716 m)."

Figure 2.4. Self-similar Velocity Profiles in the Central Upwell with Velocity Scaled on the Centerline Velocity and Radius Scaled on the Radius Corresponding to Half the Velocity for (a) Water, (b) KB Slurry, (c) $10 \mu \mathrm{m}$ Glass Beads at $5 \mathrm{wt} \%$, (d) $10 \mu \mathrm{m}$ Glass Beads at 20 $\mathrm{wt} \%$, (e) $35 \mu \mathrm{m}$ Glass Beads at $5 \mathrm{wt} \%$, and (f) $35 \mu \mathrm{m}$ Glass Beads at $20 \mathrm{wt} \%$. Numbers in legends denote elevation scaled on nozzle diameter. Curve fits include the predicted profiles of a Gaussian (Meacham et al. 2012), Squire and Trouncer (Rajaratnam 1976), Goertler (Abramovich 1963; Rajaratnam 1976), and Tollmien (Rajaratnam 1976) as described in the text. Fits with the single parameter normalized Fermi-Dirac function, Eq. (2.9), take $s_{f}$ $=0.368$ (not shown), $0.130,0.351,0.259,0.206$, and 0.385 in panels a through $\mathrm{f}$, respectively.

Figure 2.5. Jet Width $\delta_{\mathrm{c}}$ Scaled on Nozzle Diameter as a Function of Scaled Elevation for Water (Bontha et al. 2003a, b), KB Slurry (Bamberger et al. 2005), and Glass Beads of Size and Concentration Indicated (Bontha et al. 2003b). Error bars shown for KB slurry (inside red circles).

Figure 2.6. Shear Stress versus Shear Rate for Up Ramp (blue +) and Down Ramp (red circle) for KB Slurry Used in Run Sheets 3 and 4. This shows that creep flow may set in at shear rates below $\sim 0.3 \mathrm{~s}^{-1}$. The characteristic curves are (solid) for the Bingham-Papanastasiou model $\left(\tau=\tau_{o}[1-\exp (-m \dot{\gamma})]+\mu_{o} \dot{\gamma}\right.$ with a consistency of $\mu_{o}=25.75 \mathrm{mPa}$ s and the yield stress is $\tau_{o}=27.58 \mathrm{~Pa}$, and $m(\sim 1 \mathrm{~s})$ is a regularization parameter with dimensions of time [Mitsoulis 2007]) and (dashed) the ideal Bingham plastic $(m \rightarrow \infty)$. The former allows for determination 
of yielded/unyielded regions a posteriori and facilitates theoretical analysis in Section 4.

For information only (FIO). Data courtesy of Carolyne Burns of PNNL

Figure 2.7. Positions of Valeport Velocity Probes for Run Sheets (a) 1; (b) 2, 6, and 7; (c) 3; and

(d) 4 and 5. Courtesy of Korey Peterson and Christopher Chapman. Lateral/radial probe positions not verified. FIO.

Figure 2.8. Exemplary Characteristics of Test 1-8.4.3 (time stamp 075218): (a) PJM Level, PJM

Pressure, and Radar Level Average; (b) Vertical Velocities 0, 34, and 68 inches Above the

Seam; and (c) Vertical and Horizontal Velocities at 68 inches Above Seam Versus Time

from the Start of the Pulse. This test was conducted in the $8 \mathrm{ft}$ vessel with $H / D=1.0$,

$d_{o}=1.94$ in, $U_{o}=12 \mathrm{~m} / \mathrm{s}$, pulse volume fraction $(\mathrm{PVF})=0.1$ using the $\mathrm{S}$ firing sequence with

the two-part Newtonian simulant. Surface flow reported as "sustained roll-over of entire

field of view" (Bontha et al. 2015, Appendix G). FIO.

Figure 2.9. The Leading Penetration Front of a Jet Showing the Jet Core and Leading Vortex

Ring (Shekarriz et al. 1995b). The leading vortex may be stationary. Original caption: "Fig.

4 Schematic diagram showing the various flow regions within the pseudoplastic jet." Used with permission from ASME.

Figure 2.10. Exemplary Characteristics of Test 1-8.4.2 (time stamp 052539): (a) PJM Level, PJM Pressure, and Radar Level Average; (b) Vertical Velocities 0, 34, and 68 inches Above the Seam; and (c) Vertical and Horizontal Velocities at 34 inches Above Seam Versus Time from the Start of the Pulse. This test was conducted in the $8 \mathrm{ft}$ vessel with $H / D=1.0, d_{o}=1.94$ inches, $U_{o}=9.4 \mathrm{~m} / \mathrm{s}, \mathrm{PVF}=0.1$ using the $\mathrm{S}$ firing sequence with the two-part Newtonian simulant. Surface flow reported as "not near surface" (Bontha et al. 2015, Appendix G). FIO.

Figure 2.11. Exemplary Characteristics of Test 3-8.5.1 (time stamp 140349): (a) PJM Level, PJM Pressure, and Radar Level Average; (b) Vertical Velocities 0, 34, and 60 inches Above the Seam; and (c) Vertical and Horizontal Velocities at 60 inches Above Seam Versus Time from the Start of the Pulse. This test was conducted in the $8 \mathrm{ft}$ vessel with $H / D=1.0, d_{o}=1.94$ inches, $U_{o}=12 \mathrm{~m} / \mathrm{s}, \mathrm{PVF}=0.2$ using the $\mathrm{S}$ firing sequence with the KB slurry. Surface flow reported as "1-Strong" (Bontha et al. 2015, Appendix G). FIO.

Figure 2.12. Exemplary Characteristics of Test 3-8.1.44 (time stamp 032930): (a) PJM Level, PJM Pressure, and Radar Level Average; (b) Vertical Velocities 0, 34, and 60 inches Above the Seam; and (c) Vertical and Horizontal Velocities at 60 inches Above Seam Versus Time from the Start of the Pulse. This test was conducted in the $8 \mathrm{ft}$ vessel with $H / D=1.0, d_{o}=1.94$ inches, $U_{o}=8 \mathrm{~m} / \mathrm{s}, \mathrm{PVF}=0.15$ using the $\mathrm{S}$ firing sequence with the KB slurry. Surface flow reported as "5-NA" (Bontha et al. 2015, Appendix G). FIO.

Figure 2.13. Experimental Data from the $8 \mathrm{ft}$ Test Vessel with Central Upwell Velocity and Elevation Scaled on a Nozzle Velocity (see Table 2.3) and the Nozzle Diameter (1.94 inches except where listed), Respectively, for ( $a$ and $b$ ) the Two Part Simulant of Run Sheets 1 and 2 at Two Diameters, (c and d) the KB Slurry of Run Sheet 3 and Water, and (e and f) the Multipart Simulants of Run Sheet 7. Panels a, c, and e include data for which surface flow was observed (fits do not include velocities below $u_{m, c} / U_{o}<0.05$; surface flow not reported for water) and panels $b, d$, and $f$ represent data for which surface flow was not observed. Vertical line at $z / d_{o}=48$ represents the dimensionless fill height of the proposed standard high solids vessel and the geometrically scaled $8 \mathrm{ft}$ test vessel.

Figure 2.14. (a) The Local Velocity versus Turbulence Metric Described in the Text for Various Simulants at Two Probe Elevations (top 60-68 inches above seam and middle 34 inches above seam). The original Poirier-Etchells cutoff velocity of $0.50 \mathrm{ft} / \mathrm{s}(0.15 \mathrm{~m} / \mathrm{s})$ (dots) and the revised velocity cutoffs of $0.33 \mathrm{~m} / \mathrm{s}$ (dashed) and $0.42 \mathrm{~m} / \mathrm{s}$ (dash dot) for Newtonian and 
KB slurries, respectively, are shown as horizontal lines. (b) The Dimensionless

Poirier-Etchells Group Differentiated on the Surface Flow Metric Described in the Text

Figure 2.15. (a) Predicted versus Experimental Cloud Height Scaled on Nozzle Diameter with $u_{c o}=0.60$ and $0.48 \mathrm{~m} / \mathrm{s}$ for the 1.94 and 2.50 inch Nozzles, Respectively, for Tests from Run Sheets 1 and 2 Listed in Table 2.3 Compared to Fill Height/Nozzle Diameter for $d_{o}=1.94$ inches (dash, blue) and 2.50 inches (dash dot, red). (b) Predicted Cloud Height Differentiated on the Surface Flow Metric of Table 2.3 with $u_{c o}$ as in Panel a.

Figure 3.1. Depth of Virtual Origin Scaled on Nozzle Diameter Versus Jet Coefficient for a Variety of Vessels, Nozzle Diameters, and Simulants. 3.13

Figure 5.1. The Froude Number Differentiated on the Surface Flow Metric of Table 2.3 5.2

Figure 5.2. (a) Model Prediction versus Experimentally Obtained Values of the Cloud Height Scaled on Nozzle Diameter for the Two-Part Simulant of Run Sheets 1 and 2 (Table 2.3) Based on Densities from Bontha et al. (2015, Appendix G) and $h_{c}$ and $z_{o} / d_{o}$ at Surface Flow Conditions per Table 2.4 Compared to Fill Height/Nozzle Diameter for $d_{o}=1.94$ inches (dash, blue) and 2.50 inches (dash dot, red). (b) Model Cloud Height Scaled on Nozzle Diameter Differentiated on Surface Flow Metric of Table 2.3. (c) Model Cloud Height Scaled on Nozzle Diameter versus $\rho_{\max } / \rho_{\infty}$ for the Two-Part Simulant with a 1.94 inch Nozzle Diameter (blue solid: $\mathrm{Fr}=298, h_{c}=4.26, z_{o} / d_{o}=32.8$ ) and Extrapolated for the TwoPart Simulant with a 4 inch Nozzle Diameter (red dash: $\mathrm{Fr}=144, h_{c}=4.26, z_{o} / d_{o}=32.8$ ), the Six-Part Simulant with a 1.94 inch Nozzle Velocity (green dot dash: $\mathrm{Fr}=298, h_{c}=4.35$, $\left.z_{o} / d_{o}=28.7\right)$, and the Extrapolated for the Six-Part Simulant with a 4 inch Nozzle Velocity (purple double dot dash: $\mathrm{Fr}=144, h_{c}=4.35, z_{o} / d_{o}=28.7$ ). (d) Measured Minimum and Maximum Slurry Densities from Appendix G of Bontha et al. (2015) versus Nozzle Velocity with the Density Ratio Thereof.....

Figure 5.3. Centerline Velocity Scaled on Nozzle Velocity Versus Elevation Scaled on a Nozzle Diameter Comparing (a) Eq. (5.10) and (b) Eq. (2.1). See Table 5.1 for parameters used to fit panel a. Vertical lines at $z / d_{o}=36$ and 48 represent the dimensionless fill height of the $12.75 \mathrm{ft}$ vessel $\left(z / d_{o}=29-36\right.$ in Bontha et al. 2003b) and 8 or $16 \mathrm{ft}$ standard high solids vessel designs, respectively.

Figure 6.1. "Cavern" Height Data Scaled on Nozzle Diameter Versus Yield Reynolds Number with Best Fits to (a) Eq. (6.7) and (b) Eq. (6.15) with Fit Parameters in Table 6.1

Figure 6.2. Sand Jet in Laponite at Decreasing Entrance Velocities (left to right). Original caption: "Fig. 2. Example images of deformation types I to III of sand jets in Laponite gel." Reprinted with permission from Cai et al., Copyright 2012, Chinese Society of Theoretical and Applied Mechanics and American Institute of Physics.

Figure 6.3. (a) "Cavern" Height Versus Yield Reynolds Number, (b) $R_{j e t}$ Nondimensionalized on the Nozzle Diameter versus Yield Reynolds Number, and (c) $R_{j e t}$ Nondimensionalized on the Nozzle Diameter versus the Ratio of the Yield Reynolds Number to the Jet Reynolds Number for Laponite Gels at Three Vessel Sizes....

Figure 6.4. Fanning Friction Factor for a Bingham Plastic Versus the Bingham Reynolds Number (a) for $\mathrm{He}=1.610^{6}$ and $1.610^{8}$ and (b) for Experimental Data Using Kaolin Slurries from Masuyama et al. (1980). Original Caption: "Fig. 2 Relationship between $f$ and $N_{R e B}$ for kaolin slurries." Note the offset in axes between panels. Reprinted with permission.

Figure 6.5. Axial Velocity Profiles Normalized on the Centerline Velocity Versus Radial Position Normalized on the Jet Radius Determined by Linear Fitting. Experimental data from Figure $2.4 \mathrm{~b}$ compared to the $1 / 7^{\text {th }}$ law, laminar profile, and Gaussian profiles. Best fit laminar velocity profile constants are $r_{c} / R_{j e t}=0.245$ and $\tau_{o} R_{j e t} / \mu_{0} u_{c}=0.857$ 
Figure 6.6. The Square of the Ratio of the Centerline Velocity to the Nozzle Velocity Versus the Elevation Scaled on Nozzle Diameter. Nozzle velocity for $2.82 \mathrm{ft}$ and $12.75 \mathrm{ft}$ vessels was the peak average velocity (larger than average defined in Sections 2.1 and 2.4). Data from the $2.82 \mathrm{ft}$ vessel taken off center, which reduces values on the $\mathrm{y}$-axis by a factor of $f_{c}^{2}$, and are included only to inform consideration of predicted linearity. The vertical line represents the nominal fill height of the 8 or $16 \mathrm{ft}$ vessels; the other vessels had a dimensionless fill height of 29 to 36 .

Figure 7.1. Central Upwell Velocity 0, 34, and 60 inches Above the Seam for a Six-Part Simulant at Velocities of (a) $9.5 \mathrm{~m} / \mathrm{s}$ (time stamp 152733), (b) $10.5 \mathrm{~m} / \mathrm{s}$ (154932), (c) 11.5 $\mathrm{m} / \mathrm{s}(085206)$, (d) $12 \mathrm{~m} / \mathrm{s}(091401)$, (e) $13 \mathrm{~m} / \mathrm{s}$ (143502), and (f) $15 \mathrm{~m} / \mathrm{s} \mathrm{(102228)} \mathrm{with}$ $\mathrm{PVF}=0.2, d_{o}=1.94$ inches, $f_{R}=0.6$. Surface flow observed for $U_{o} \geq 12 \mathrm{~m} / \mathrm{s}$

Figure 7.2. Nozzle Velocity Divided by Centerline Velocity Versus Distance from Nozzle Scaled on Nozzle Diameter from Shekarriz et al. (1995b). Original caption: "Fig. 9 Instantaneous center-line velocity within the terminating regions shown in Figs. 7 and 8." Reprinted with permission from ASME.

Figure 7.3. Elevation Scaled on Nozzle Diameter Versus Time Scaled on the Jet Time (ratio of the nozzle diameter to the nozzle velocity) for (a) Water in the $12.75 \mathrm{ft}$ Vessel and (b) Water in the $8 \mathrm{ft}$ Vessel at $12 \mathrm{~m} / \mathrm{s}$, (c) for Glass Particles (10 and $35 \mu \mathrm{m}$ Glass Beads at 5 and $20 \mathrm{wt} \%$ ) in the $12.75 \mathrm{ft}$ Vessel, (d) for the Six-Part Simulant in the $8 \mathrm{ft}$ Vessel at Various Velocities, and (e) the Multipart Simulants in the $8 \mathrm{ft}$ Vessel at $15 \mathrm{~m} / \mathrm{s}$, (f) the $\psi_{2,1} / \psi_{1,1}$ Ratio versus Nozzle Velocity for the Multipart Simulants in the $8 \mathrm{ft}$ Vessel

Figure 7.4. The Ratio of the Upwell Time to the Drive Time Differentiated on the Surface Flow Metric of Table 2.3. When this time ratio is small, surface flow should be achieved, and when it is large, the upwell does not reach the surface before the jet turns off.

Figure 8.1. Velocity Profiles Along the Wall 0 inches (red, Valeport 0578), 34 inches (blue, Valeport 0577), and 60 inches (green, Valeport 0576) Above the Seam for Test 7-8.6.2 (time stamp 103104) for the Three-Part Simulant with $U_{o}=15 \mathrm{~m} / \mathrm{s}, d_{o}=1.94$ inches, $\mathrm{PVF}=0.2$, and $\mathrm{S}$ Firing. Negative probe velocities represent vertically upward flow.

Figure 8.2. Depth of the Virtual Origin below the Seam/Tangent Scaled on Nozzle Diameter, $z_{o} / d_{o}$, Versus the Wall Jet Coefficient, $h_{w}$, for the Three-Part and Six-Part Simulants

Figure 9.1. Fluid Velocities in the $8 \mathrm{ft}$ Vessel in Tests (a) 3-8.5.1 (time stamp 140349) with $U_{o}=12 \mathrm{~m} / \mathrm{s}$ and (b) 3-8.1.44 (time stamp 032930) with $U_{o}=7.9 \mathrm{~m} / \mathrm{s}$ with Nozzle Velocities Both Measured 34 inches Above the Seam and 18.9 inches and 1.1 inches From the Vessel Center with Valeport Probes 0576 and 0580, respectively. A 2.25 s delay in processing the Valeport probe information may affect the timing of these curves relative to the pulse start (Bontha et al. 2015). 


\section{Tables}

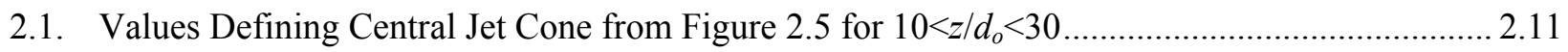

2.2. Ambient Physical Properties of Simulants Used in the $8 \mathrm{ft}$ Test Vessel..................................... 2.12

2.3. Summary of Test Operating Conditions and Reported Observations for Tests Evaluated ............ 2.24

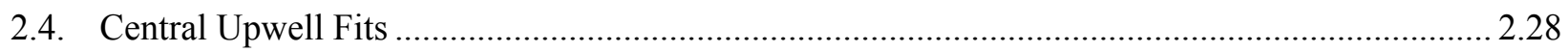

3.1. Constants Derived from Radial Distributions ............................................................................ 3.3

3.2. Bulk Mass Average Velocity Due to Particle Settling for Run Sheet 7 ....................................... 3.9

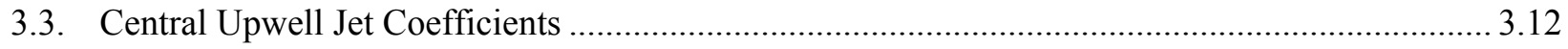

4.1. Constants Derived from Radial Distributions ............................................................................ 4.15

5.1. The Values Used to Fit Eq. (5.10) in Figure 5.3a .................................................................... 5.9

6.1. Best Fit Parameters for "Caverns" Measured in Laponite ............................................................. 6.5

6.2. Best Fit Parameters for "Caverns" Measured in KB Slurry .......................................................... 6.9

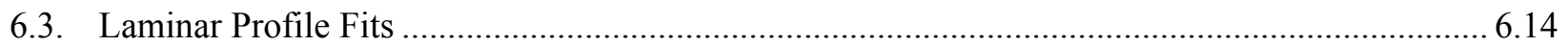

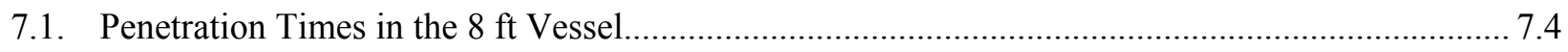

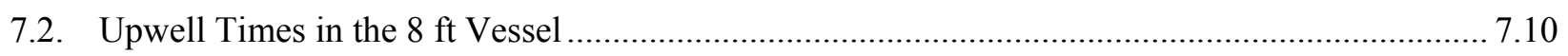

9.1. Projected Volumes of Mixing at Surface Flow Achieved by the Central Upwell Alone per Pulse in a $16 \mathrm{ft}$ Vessel 



\subsection{Introduction: Jet Physics in the Mixing Vessels}

Interest in the performance of pulse jet mixers (PJMs) in the high solids vessels of the Hanford Tank Waste Treatment and Immobilization Plant (WTP) leads to consideration of the physics of flow within these vessels. Slurry suctioned into a PJM at the end of the prior pulse is pneumatically driven from the PJM toward the bottom of the vessel, forming a jet at the beginning of a new pulse. A jet is defined as "a stream of material that travels for many diameters in a nearly constant direction" (Birkhoff and Zarantonello 1957). The jet front quickly traverses the distance from nozzle outlet to the bottom of the vessel. The vessel bottom is either spherical for the $8 \mathrm{ft}$ test vessel or elliptical for the planned $16 \mathrm{ft}$ standard high solids vessel. The flow spreads out radially along the bottom of these vessels with some portion of the flow headed toward the vessel wall and the remainder flowing toward the center. As the centrally directed flow proceeds along the vessel bottom, it approaches the center and collides with neighboring jets to create a central upwell. This upwell flow both in the center and along the vessel wall is the primary consideration herein (see Figure 1.1).

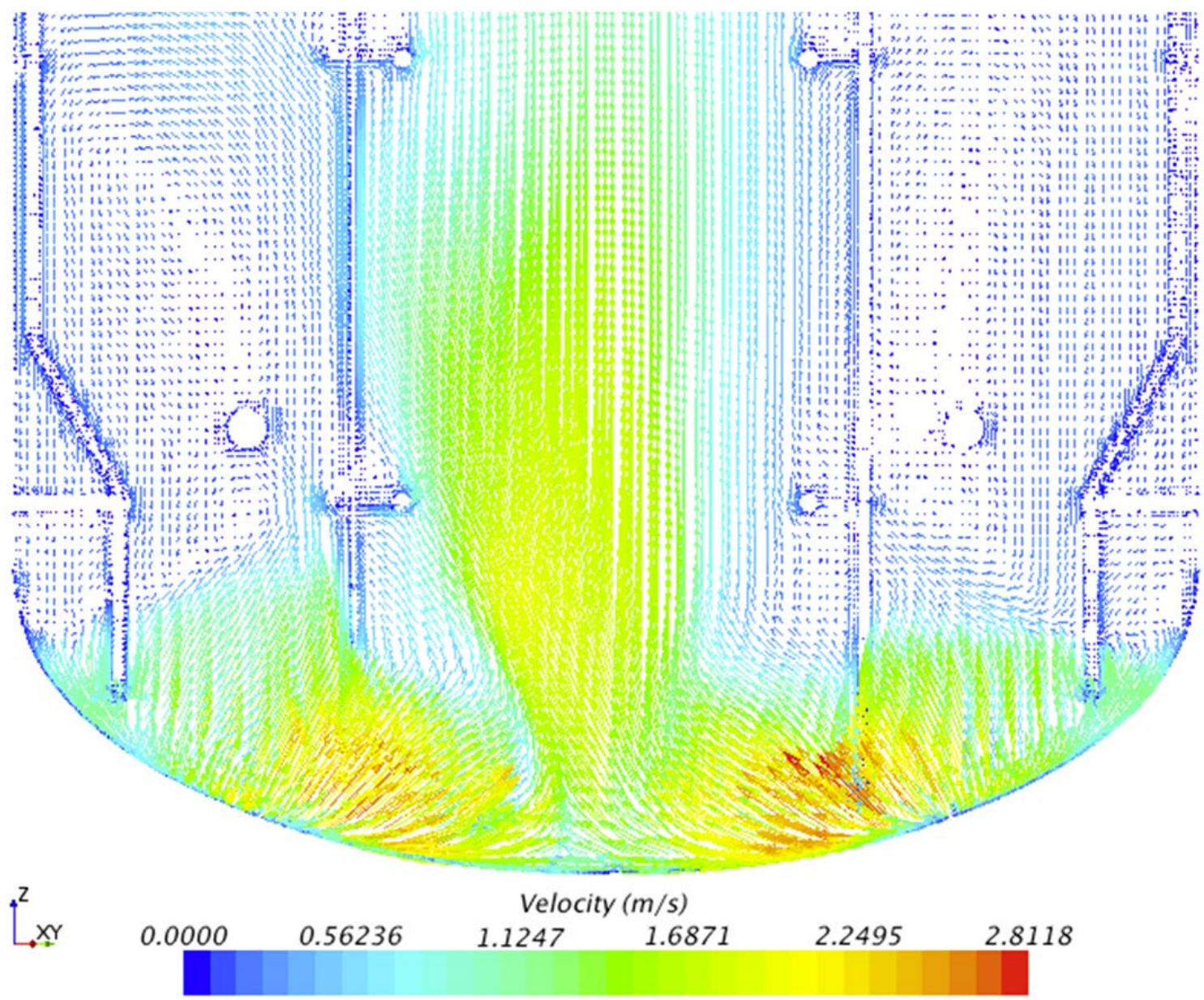

Figure 1.1. Visualization of Flow Within a Pulse Jet Mixed Vessel Between PJMs, Highlighting the Sheared Regions Including the Flow Along the Vessel Bottom and the Central Upwell. This report evaluates the central and wall upwells. Simulation for $16 \mathrm{ft}$ vessel with $U_{o}=12 \mathrm{~m} / \mathrm{s}$, $d_{o}=4$ inches, $\mu_{o}=30 \mathrm{mPa}$ s, and $\rho=1200 \mathrm{~kg} / \mathrm{m}^{3}$ without solids. Asymmetry in central upwell due to suction pipe (not shown) blocking upward momentum. Courtesy of Kurt Recknagle. 
The physics of jet flow pertaining to the primary downward flow and second radial flow along flat vessel bottoms has been considered in some detail. The initial downward flow is governed by a developing circular free jet where the plug flow of the jet core gives way to the shear forces that induce vortices and an approximately Gaussian velocity distribution. This process is complete within 4 to 8 nozzle diameters (the nozzle diameter remains the essential characteristic length of a jet throughout its traverse). This jet is termed a "free jet" because surface and body forces remain negligible relative to jet momentum and has been described by numerous authors (see Bird et al. 2002; Rajaratnam 1976). As the jet impinges on the vessel bottom, it develops a pressure that redirects the flow radially (Beltaos 1975; Beltaos and Rajaratnam 1977; Phares et al. 2000). Radial jets, so named because the flow spreads out radially with respect to the impinging jet centerline, experience shear forces along the vessel bottom and lose their dependence on nozzle conditions (but retain the nozzle velocity and diameter as characteristic scales). Yet, when the flows of impinging nozzles collide, the physics that govern the central upwell has received considerably less attention. Kuhn et al. (2013) considered how a pair of impinging jets might interact to form a planar upwell and also suggested that the central upwell may be described as a free jet with a velocity that decays with elevation. How the central upwell is affected by multiple PJMs, significant multicomponent solids loadings, and generalized Newtonian or non-Newtonian rheologies remains unexplored from a jet physics perspective.

Therefore, this report considers mixing phenomena ${ }^{1}$ above the vessel bottom, drawing heavily on both jet physics and data at the largest scales available to obtain the necessary insight and address the following three questions.

1. Is the central upwell a hydrodynamic jet? Jet physics models impose specific and unescapable consequences on the upwell, and free jets that reach and break through to the upper fluid surface represent preferred mixing scenarios using PJMs. Section 2 argues that the central upwell may be described as an approximately circular free jet with velocity that decays as the inverse of the elevation, for nozzle velocities that are sufficiently large. Large-scale data sets including both historical data from a $12.75 \mathrm{ft}$ vessel in Sections 2.1 and 2.2 (Bontha et al. 2003a, b; Bamberger et al. 2005) and newly analyzed data from the $8 \mathrm{ft}$ vessel in Sections 2.3 and 2.4 concur with this perspective. Section 2.5 considers the minimum jet velocity necessary for the jet to be a turbulent free jet as the Poirier-Etchells cutoff velocity. Section 3 derives conservation of momentum arguments that relate PJM operating conditions to the functional form of the velocity decay for a free jet.

2. Under what conditions does the central upwell change flow regimes from a central free jet to something else, and what are the alternatives from a jet physics perspective? Section 4 presents the governing differential equations for both momentum and species conservation in which both

\footnotetext{
${ }^{1}$ The terms mixing, blending, and shearing have an abundance of definitions. Blending may refer to homogenization of miscible liquids and mixing to homogenization of solids. Alternatively, mixing may refer to thermodynamic processes in which one body of molecules adjacent to another body of another type of molecules form a single body by exchange of energies, which is essential for chemical reactions (e.g., neutralization, leaching, etc.) to occur. This typically happens at length scales smaller than the Kolmogorov length scale within its own time scale that may be longer or shorter than time scales for jet convection and chemical reactions. Here the terms mixing, blending, and shearing are used interchangeably herein and refer specifically to regions of the vessel in which substantial jet flows occur. These flows include both the convective flow and turbulent vortices at length scales larger than the Kolmogorov length scale that are essential to any significant mixing, blending, or chemical reactions by any definition.
} 
negative buoyancy and shear forces participate. Sections 5 through 7 then use these equations to predict how negative buoyancy, yield stresses, and jet transience affect the flow. Section 5 evaluates the Froude number as a predictor of surface flow and derives a new four-parameter cloud height model that compares favorably to experimental data. Section 6 considers the influence of yield stresses on expanding turbulent jets, confined turbulent jets, and confined laminar flows. For each, a critical elevation is predicted at which jet momentum depletes that compares favorably to experimental "cavern" height data. Section 4.3 shows that these are each limiting cases of a gradual transition from a turbulent expanding flow to a confined laminar flow. When the jet expands the critical elevation depends on the yield Reynolds number (a ratio of jet momentum to fluid yield stresses) to the $1 / 2$ power, and when the jet is confined the critical elevation depends linearly on the yield Reynolds number. This analysis of the central upwell alone lays essential groundwork for complete analysis of mode three mixing (Bamberger et al. 2005). Section 7 considers jet celerity (i.e., the rapidity or swiftness of jet front penetration) and shows that this may be a governing consideration in jet flow reaching the surface.

3. What are the implications of jet physics for vessel mixing? The vessel is mixed by both the annular wall jet and the central upwell. Section 8 shows that flow along the vessel wall is weaker and sluggish. Section 9 then evaluates the implications of upwell flows for vessel mixing on a per pulse basis. Vessel mixing may also be assisted by the leading vortex that defines the leading edge of jet penetration that extends beyond the boundaries of the jet and by down flow along the vessel wall.

In summary, this analysis shows that achieving surface flow for a Newtonian simulant may depend on the ratio of the virtual origin to the nozzle diameter of the jet, the Froude number, and ratio of the density at the base of the upwell to the density far from the jet centerline, in addition to factors that do not depend on geometric scaling. For a non-Newtonian simulant, surface flow is primarily governed by factors that do not depend on geometric scale but may be modestly influenced by the jet Reynolds number through the effective wall stresses and the ratio of the virtual origin to the nozzle diameter. This report concludes with a gap analysis.

This analysis is limited to the upwells in the center of the vessel and along the vessel wall. Analysis of bottom motion, downward flow peripheral to the central jet, or downward flow that derives from central upwell flows that cross the upper surface and then move down the vessel walls remains outside the scope of this analysis. Particles that are mobilized by bottom motion but are not elevated into the central upwell are not incorporated in the analysis to follow.

\subsection{Scope}

Pacific Northwest National Laboratory (PNNL) was asked to provide a Basic Research technology level report that evaluated the physics of the upwell flow in pulse jet mixed systems. This understanding of the central upwell would be used to evaluate PJM performance in small-scale testing and full-scale PJM vessel performance with Newtonian and non-Newtonian simulants. This report developed models that can be used in conjunction with full-scale testing to evaluate the central upwell flow within the vessel. The review of prior testing and literature did not provide a simple method for direct scaling of central upwell performance from small-scale testing to full-scale vessels. 
This application is for PJMs arranged in circular/distributed arrays in vessels with dished bottoms. The circular arrays contain no central PJM and the bulk flow patterns are dominated by a central upwell within the PJM array and downward flow in the annular region surrounding the circular array.

The scope for this report is provided in the TP-WTPSP-132 Test Plan, Section 5.1, tasks i and $\mathrm{j}$, with the contractual direction authority for this report provided in Section 3.3.1 of Hazen (2014).

\subsection{Quality Requirements}

PNNL complies with the requirements found in the following standards and implements them in their Waste Treatment Plant Support Program (WTPSP) Quality Assurance Program:

- ASME NQA-1-2000, Quality Assurance Requirements for Nuclear Facility Applications, Part I, Requirements for Quality Assurance Programs for Nuclear Facilities

- ASME NQA-1-2000, Part II, Subpart 2.7, Quality Assurance Requirements for Computer Software for Nuclear Facility Applications

- ASME NQA-1-2000, Part IV, Subpart 4.2, Guidance on Graded Application of Quality Assurance (QA) Requirements for Nuclear-Related Research and Development.

Records will be stored as hardcopy records in a 2-hour fire-rated container.

This project recognizes that quality assurance applies in varying degrees to a broad spectrum of research and development $(\mathrm{R} \& \mathrm{D})$ in the technology life cycle. The R\&D elements in the technology life cycle are listed below. The WTPSP uses a graded approach for the application of the quality assurance controls such that the level of analysis, extent of documentation, and degree of rigor of process control are applied commensurate with their significance, importance to safety, life cycle state of work, or programmatic mission. The technology life cycle is characterized by flexible and informal quality assurance activities in basic research, which becomes more structured and formalized through the applied R\&D stages.

- Basic Research: Basic Research consists of research tasks that are conducted to acquire and disseminate new scientific knowledge. During Basic Research, maximum flexibility is desired in order to allow the researcher the necessary latitude to conduct the research.

- Applied Research: Applied Research consists of research tasks that acquire data and documentation necessary to ensure satisfactory reproducibility of results. The emphasis during this stage of a research task is on achieving adequate documentation and controls necessary to be able to reproduce results.

- Development Work: Development Work consists of research tasks moving toward technology commercialization. These tasks still require a degree of flexibility and a degree of uncertainty exists in many cases. The role of quality on Development Work is to make sure that adequate controls to support movement into commercialization exist.

- Research and Development Support Activities: Support activities are those that are conventional and secondary in nature to the advancement of knowledge or development of technology, but allow the primary purpose of the work to be accomplished credibly. An example of a support activity is 
controlling and maintaining documents and records. The level of quality for these activities is the same as for Development Work.

This report was conducted at the Basic Research technology level. 



\subsection{The Central Upwell is a Free Jet-At Least Sometimes}

This analysis begins with the premise that the PJM mixed vessels above the vessel bottom achieve optimal performance when the upwells flow like circular free jets. This report then evaluates whether the upwells follow free jet physics and determines the conditions under which the upwells do not flow like free jets. The central upwell must satisfy several requirements to be considered a free jet. First, it must travel for several diameters in a nearly constant direction. Second, the influence of surface and body forces must be negligible, hence the term "free." Third, its velocity must decay with the inverse of elevation. Fourth, the radial velocity profile should be self-similar if not approximately Gaussian. This section demonstrates that the central upwell satisfies these requirements (at least sometimes) as discussed below.

The historical central upwell velocity data in both water and kaolin-bentonite (KB) clay are shown in Figure 2.1, with the local upwell velocity along the centerline of the jet, $u_{m, c}$, scaled on the nozzle velocity, $U_{o}$, and the elevation, $z$, scaled on the nozzle diameter, $d_{o}$. This scaling was selected because free jet data collapse onto a single curve with this scaling (Rajaratnam 1976). This universal scaling is consistent regardless of rheology (Newtonian or generalized Newtonian) so long as the jet remains free (i.e., negligible surface and bulk forces), and Bird et al. (2002) in their Table 5.1-1 show that this scaling is true for both turbulent and laminar circular jets. The curve is given as

$$
\frac{u_{m, c}}{U_{o}}=h_{c}\left(\frac{z+z_{o}}{d_{o}}\right)^{-1}
$$

where $z_{o}$ is distance from the vessel bottom to the virtual origin, with the virtual origin located at the vertex of the cone defined by the expanding jet (Kuhn et al. 2013; Rajaratnam 1976), and $h_{c}$ is a jet coefficient that depends strongly on geometry (e.g., numbers of PJMs) and more modestly on density gradients and so forth as derived below. ${ }^{1}$ Therefore, fitting velocity data with a power law will yield an exponent of -1 regardless of nozzle Reynolds number. On a log-log plot such a power law fit would be a straight line with a slope of -1 , though the virtual origin may induce curvature.

\subsection{Analysis of Historical Centerline Velocity Data}

Equation (2.1) was evaluated using historical data with three or more PJMs. Testing with four PJMs was performed by Bontha et al. (2003a, b) and Bamberger et al. (2005) in the large tank test stand (LTTS) with a tank diameter of $12.75 \mathrm{ft}$ and in a geometrically downscaled equivalent (4.53x) with a diameter of $2.82 \mathrm{ft}$. Bontha et al. (2003a, Appendix D) evaluated the vertical upwell velocity in water along the vessel centerline in the LTTS using Valeport velocity probes. Figure 2.1a shows this data with the centerline velocity divided by the nozzle velocity versus elevation divided by the nozzle diameter or with scaled velocity versus scaled elevation. The centerline velocity was averaged from the approximate leading inflection point to the approximate end of pulse with a nozzle velocity of $8.86 \mathrm{~m} / \mathrm{s}$. Subsequently, Bontha et al. (2003b) further evaluated the upwell velocity in water in the LTTS. Data were tabulated electronically (see file $021108 \mathrm{~T}$ for centerline velocity measurements per Table 4.1 of Bontha et al.

\footnotetext{
${ }^{1}$ Although prior work has called $h_{c}$ a constant or a jet constant, it is termed a coefficient herein because of its dependence on vessel and flow properties.
} 
2003b), and Figure 2.1a presents the average centerline velocity data using Valeport probes in the vertical direction across many pulses with a nozzle velocity of $8.76 \mathrm{~m} / \mathrm{s}$. Bontha et al. (2003b) also evaluated the vertical velocity profile of 10 and $35 \mu \mathrm{m}$ glass beads at 5 and $30 \mathrm{wt} \%$ in the LTTS with four PJMs (tabulated in files 02115C, 02115J, 021121B, 021204C, 021204J, 021212C, 021212I as given in Tables 5.2, 5.5, 5.8, and 5.11 of Bontha et al. 2003b) as in Figure 2.1b. ${ }^{1}$ Bamberger et al. (2005) evaluated the vertical upwell velocity in KB slurry in both the $12.75 \mathrm{ft}$ and $2.82 \mathrm{ft}$ vessels. Velocities were measured using ultrasound probes (see Tables 4.21 and 4.22 and Sections 4.7, 5.4, A.5, and B.3.6 of Bamberger et al. 2005) and scaled on the reported peak average velocity. These data from the $12.75 \mathrm{ft}$ vessel were collected 0.5-5.1 inches off vessel center and in the $2.82 \mathrm{ft}$ vessel 2.5 inches off centerline.
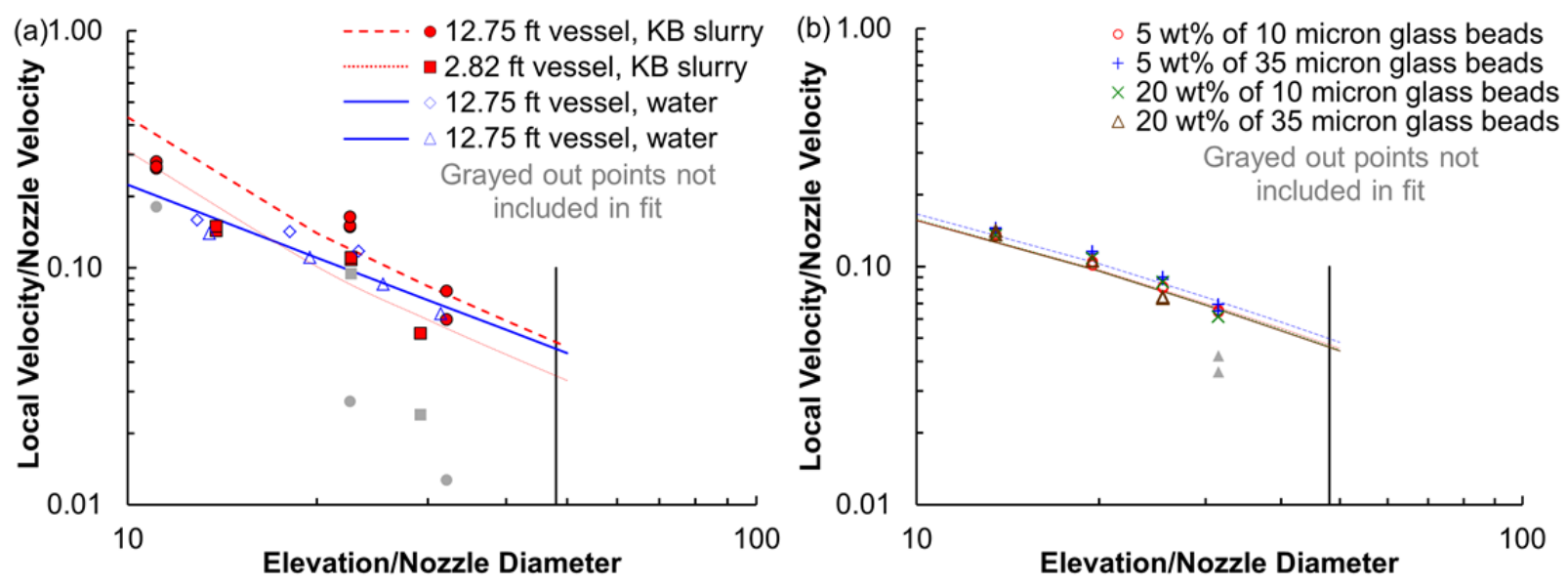

Figure 2.1. Compilation of the Historical Central Upwell Velocity Data with Upwell Velocity and Elevation Scaled on a Nozzle Velocity and the Nozzle Diameter, respectively. Curve fits from Eq. (2.1). Vertical line at $z / d_{o}=48$ represents the dimensionless fill height of the proposed standard high solids vessel and the geometrically scaled $8 \mathrm{ft}$ vessel for the 1.94 inch nozzle. The nominal fill height of the 2.82 and $12.75 \mathrm{ft}$ vessels was at $z / d_{o}=29-36$.

The resulting data are presented in Figure 2.1a for water (blue) and KB slurry (red). The data were fit by inverting Eq. (2.1) as

$$
\frac{U_{o}}{u_{m, c}}=\frac{1}{h_{c}} \frac{z}{d_{o}}+\frac{1}{h_{c}} \frac{z_{o}}{d_{o}}
$$

which shows that a plot of $U_{o} / u_{m, c}$ versus $z / d_{o}$ should be a straight line. ${ }^{2}$ The inverse of the slope is $h_{c}$ and the intercept multiplied by $h_{c}$ is $z_{o} / d_{o}$, the scaled virtual origin depth. Fitting the data accordingly gives $h_{c}=2.17$ and $z_{o} / d_{o}=-0.318$ for water (blue solid), $h_{c}=1.50$ and $z_{o} / d_{o}=-5.19$ for the KB slurry in the $2.82 \mathrm{ft}$ vessel (red dotted), and $h_{c}=2.07$ and $z_{o} / d_{o}=-5.19$ for the KB slurry in the $12.75 \mathrm{ft}$ vessel (red dashed). These fits are for the higher velocities reported (Bamberger et al. 2005) (>10 m/s in the $2.82 \mathrm{ft}$ vessel and $>6 \mathrm{~m} / \mathrm{s}$ in the $12.75 \mathrm{ft}$ vessel) as slow velocities (in grey solid symbols) may represent a different flow

\footnotetext{
${ }^{1}$ Tests with only two PJMs were also performed, which should induce a planar fan jet along the vessel centerline with scaled velocity decreasing with scaled elevation to the $-1 / 2$ power (Rajaratnam 1976) as anticipated by Kuhn et al. (2013). Such analysis may be relevant when only two PJMs are operational in the WTP Pretreatment Facility. This report focuses on systems with three or more PJMs for which a non-planar central upwell is anticipated.

${ }^{2}$ More advanced fitting techniques are available to minimize fitting error but are not necessary for scaling analyses.
} 
regime (see Sections 4 through 7). ${ }^{1}$ Data within entrance/transition regions $\left(z / d_{o}<10\right)$ have been excluded from the fits because the velocity may not be sufficiently developed there, and the water data of Bontha (2003a) at the highest elevation were excluded because of observed crossflow transverse to the axial jet velocity (see Bontha et al. 2003b, Figure 4.5). Similarly, the data for glass beads have been fit with $h_{c}=2.55$ and $z_{o} / d_{o}=6.31$ for $10 \mu \mathrm{m}$ beads at $5 \mathrm{wt} \%$ (red dotted), $h_{c}=2.48$ and $z_{o} / d_{o}=5.67$ for $10 \mu \mathrm{m}$ beads at $20 \mathrm{wt} \%$ (green short dash), $h_{c}=2.69$ and $z_{o} / d_{o}=6.19$ for $35 \mu \mathrm{m}$ beads at $5 \mathrm{wt} \%$ (blue long dash), and $h_{c}=2.46$ and $z_{o} / d_{o}=5.79$ for $35 \mu \mathrm{m}$ beads at $20 \mathrm{wt} \%$ (brown solid). Similarly, data within entrance/transition regions $\left(z / d_{o}<10\right)$ and for the $35 \mu \mathrm{m}$ glass beads at $20 \mathrm{wt} \%$ at the highest elevation due to a developing cloud height have been excluded. The implication of these data is that flow within the central upwell does follow the free jet profile for both Newtonian and generalized (i.e., non-Newtonian) rheologies for at least some operating conditions.

This plot has significant implications for scaling between the $8 \mathrm{ft}$ and $16 \mathrm{ft}$ vessels. The nominal fill height of both vessels is located as high as an elevation-to-nozzle-diameter ratio of 48 (i.e., $16 \mathrm{ft} / 4$ inches), denoted on Figure 2.1 by the vertical line. Using a larger nozzle diameter of 5 inches in the proposed standard high solids vessel, for example, places the nominal fill height at only $z / d_{o}=38.4$. If 1) the data for the $8 \mathrm{ft}$ vessel fall on (or are parallel to) one of these curves for free jets for both Newtonian and non-Newtonian simulants, 2) the free jet curves (lines in the log-log plots) can be reasonably extrapolated to 48 in the time allotted, ${ }^{2}$ and 3 ) the flow regime does not change between scales, then these curves may be used to 1) predict that the breakthrough velocity (i.e., the projected velocity at the fluid surface $)^{3}$ will be greater than zero at the vessel surface, and 2) assert confidence that the $16 \mathrm{ft}$ vessel will mix, at least within the zones of influence of the jets as defined by at least the jet radius (see Section 9). The first two conditions may be resolved by evaluating data from the $8 \mathrm{ft}$ vessel testing and may be confirmed with the $16 \mathrm{ft}$ vessel testing. The last condition is analyzed in Sections 4 through 7 .

\footnotetext{
${ }^{1}$ Some of the jets may also have attached, which could affect velocity as a function of elevation. This becomes more problematic at higher elevations within the mixing vessels. Agelin-Chaab and Tachie (2011) show that the scaled velocity decays with scaled distance from the nozzle with a power law exponent between -1.15 and -1.21 , which may explain the slight dropoff of the KB slurry data from the expected curve of around $z / d_{o} \sim 30$. Representations of an off-center breakthrough region in Appendix A of Bamberger et al. (2005) suggest jet attachment to the PJMs or to other vessel internals. Similar trends may be anticipated in the current vessels of interest. Yet, even though the attached jet flow regime is fundamentally distinct from the free jet and very likely to be present in 8 and $16 \mathrm{ft}$ vessels, the $\sim 20 \%$ change in power law exponent from free to attached jet remains modest and this flow regime will not be analyzed further herein. However, minimizing vessel internals where feasible may modestly improve mixing by averting the attached jet flow regime.

${ }^{2}$ The actual surface of the liquid exceeds the nominal fill height, depending on the volume of the PJMs as defined by their internal diameters and the vertical difference between the nominal fill height and the lowest liquid level in the PJM. For the $8 \mathrm{ft}$ test vessel, the upper liquid surface reached 90.0 to 93.5 inches from the seam or 103.9 to 107.4 inches from the vessel bottom (email from Christopher Chapman, 4/29/2015 at 9:32 am) so that $z / d_{o}=53.6-55.4$ for the 1.94 inch nozzle and $z / d_{o}=41.6$ to 43.0 for the 2.50 inch nozzle. Some extrapolation of the jet curve to the liquid surface remains necessary because obtaining reliable data from resubmerging velocity probes remains challenging. The advantage of this approach is that the extrapolation remains on a firm physical basis.

${ }^{3}$ The breakthrough velocity is the velocity that the jet would have at the surface. It is a projection because other forces, including fountains and varicose instabilities, may alter the path of the jet immediately at the surface. Nevertheless, if the projected jet velocity is significant at the fluid surface, flow reaches the vicinity of the surface and blending within the jet's zone of influence may be reasonably asserted up to the vessel surface.
} 


\subsection{Analysis of Historical Radial Velocity Distribution Data}

Equation (2.1) is derived based on self-similar velocity profiles (Rajaratnam 1976; Bird et al. 2002), which define a jet radius. A jet is self-similar if the axial velocity as a function of radius retains the same functional form or shape as the jet progresses in the axial direction. When scaled properly (i.e., the axial velocity on the nozzle velocity and the radial distance on the radial location of half the centerline velocity), the data at different distances from the jet nozzle should collapse onto a single curve.

Figure 2.2 and Figure 2.3 show the axial velocity contours as a function of radial position for a jet in water at multiple elevations in the $12.75 \mathrm{ft}$ vessel. These plots were generated by measuring the velocities at multiple, specific locations and then interpolating to generate the contour maps (Bontha et al. 2003a, b). Even though these central upwells were produced by four PJMs located outside the viewable area, these figures suggest that the velocity profile becomes approximately circular, ${ }^{1}$ presumably after an entrance region below the elevations represented in the figures. With additional PJMs (e.g., from four to six), the circularity of the profiles may be expected to improve. In addition, Bontha et al. (2003a, b) describe other reasons why the velocity profiles may be asymmetric. ${ }^{2}$

\footnotetext{
${ }^{1}$ In the absence of attachment, all jets become circular jets regardless of the shape of the entry region or nozzle form. Bejan et al. (2014) argue that the transition from a turbulent slot jet to a turbulent circular jet is accomplished over a characteristic distance of $9.6 L$, where $L$ is the width of the slot. Rajaratnam (1976, p. 272) likewise indicates that a square jet behaves like a round one within 10 nozzle diameters.

2 "For example, since the JPPs regulating the airflow to the pulse tubes were not identical, the actual drive times were not identical. In addition, the 4 PJMs were not situated exactly in the center of each quadrant of the tank (one was closer to the wall than the others). Also, the pulse tube nozzles were not exactly at the same distance from the tank floor. Finally, there were two tie beams half way into the tank, which were used to provide additional support to the pulse tubes (these tie beams could not be removed during the testing due to structural stability concerns.)" (Bontha et al. 2003a, p. 5.16). "In all of these figures, the pulse tubes A, B, C, and D are located in the NW, SW, $\mathrm{SE}$, and NE directions, respectively, at a radial position of $\sim 4.25 \mathrm{ft}(\sim 1.2954 \mathrm{~m})$. Although the geometric dissimilarity (presented in Section 3.0) still causes some asymmetry in the flow, the differences in the individual pulse tube operation also contributes to the asymmetric flow. This is illustrated in Figure 4.6 in terms of the cycle averaged liquid levels in the pulse tubes. It can be seen from this figure that pulse tubes B and D drive much harder than pulse tubes A and C." (Bontha et al. 2003b, p. 4.2).
} 

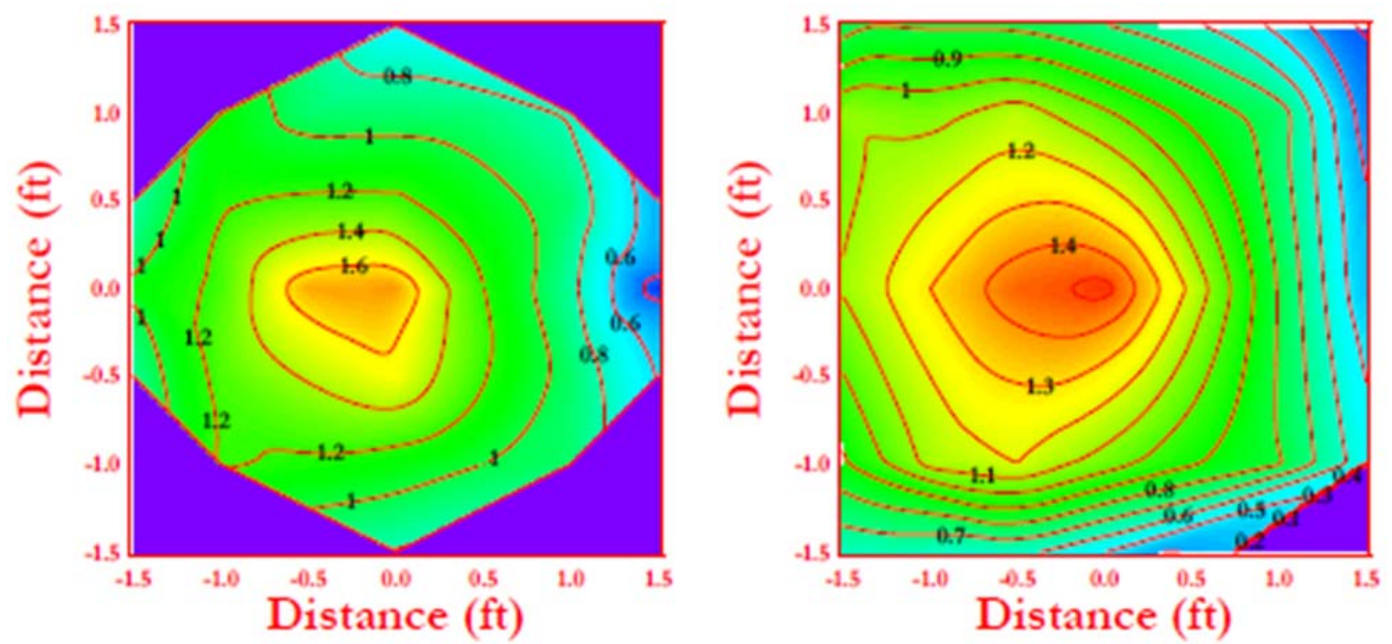

Figure 2.2. The Upwell Velocity as a Function of Radial Position in Water in the $12.75 \mathrm{ft}$ Diameter Test Vessel in the LTTS in PNNL 336 Building (Bontha et al. 2003a). PJMs located in the corners outside the field of view. Original caption: "Figure 5.13. Contour Maps of the Maximum Velocities Measured in During the Large Tank Hydrodynamic Tests: (a) $1.308 \mathrm{~m}$ (51.5 in) Horizontal Plane and (b) 1.842 m (72.5 in) Horizontal Plane."
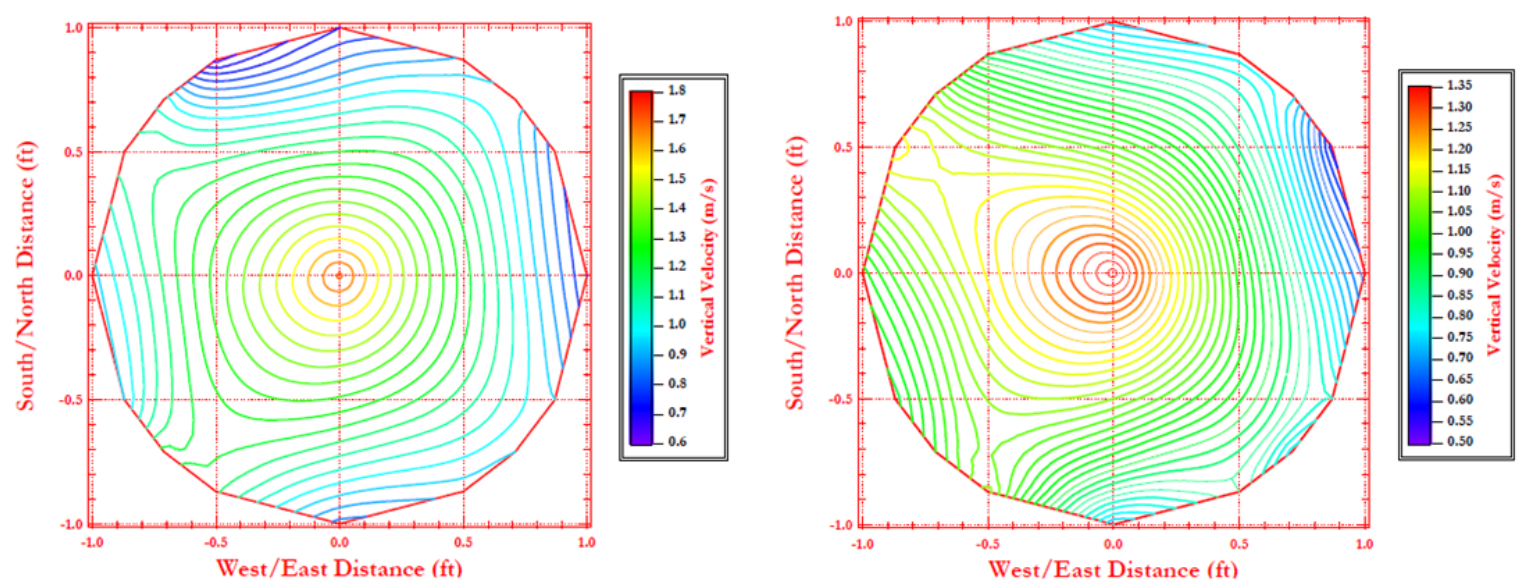

Figure 2.3. The Upwell Velocity as a Function of Radial Position in Water in the $12.75 \mathrm{ft}$ Diameter Test Vessel in the LTTS in PNNL 336 Building (Bontha et al. 2003b) Measured at the Centerline and Vertices of the Bounding Polygon. The pulse tubes are located in the corners outside the field of view. Original captions: (left) "Figure 4.2. Contour Map Showing the Fluid Velocities in a $2 \mathrm{ft}$ Diameter Core Around the Center of the Tank and an Elevation of 30 inches $(0.762 \mathrm{~m})$ " and (right) "Figure 4.3. Contour Map Showing the Fluid Velocities in a $2 \mathrm{ft}$ Diameter Core Around the Center of the Tank and an Elevation of 54 inches (1.3716 m)."

Despite the uncertainties, Figure 2.4a shows that the profiles in Figure 2.2 are indeed self-similar because the scaled data from multiple locations overlap each other indistinguishably. This figure was prepared by finding the velocity as a function of radius from the image center along the eight compass directions (cardinals and primary intercardinals) and estimating the centerline velocities to be 1.7 and $1.55 \mathrm{~m} / \mathrm{s}$. The radial location where the local velocity is half the centerline velocity was determined by linear fitting and used to scale the $x$-axis of Figure 2.4. This figure also shows various predictions of the 
self-similar velocity profiles. In each case, the axial velocity, $u$, as a function of both axial and radial coordinates, $z$ and $r$, respectively, is

$$
u(z, r)=u_{m, c} f_{c}\left(\eta_{c}\right)
$$

where $u_{m, c}$ is the axial velocity along the centerline of the jet, $f_{c}$ is a functional form defined by this equation and elucidated below as a function of

$$
\eta_{c}=r /\left[\beta_{c}\left(z+z_{o}\right)\right]=r / \delta_{c},
$$

where $\beta_{c}$ characterizes the rate of entrainment or the radial decay of the central upwell and $\delta_{c}$ is the jet width where the local velocity falls to half the jet centerline velocity. Perhaps the most convenient functional form for $f_{c}$ for a free jet is approximately Gaussian,

$$
f_{c}\left(\eta_{c}\right)=\operatorname{Exp}\left[-\operatorname{Ln}(2) \eta_{c}^{2}\right]
$$

modified from Shekarriz et al. (1995a) and Wiegel (1964) as referenced by Meacham et al. (2012) with the $\operatorname{Ln}(2)$ so that $f_{c}(1)=1 / 2$ as required for uniform scaling. For compound circular jets, where the fluid parallel to the jet also has a finite velocity in the axial direction, Rajaratnam (1976) recommends the cosine expression of Squire and Trouncer,

$$
f_{c}\left(\eta_{c}\right)=\frac{1}{2}\left(1+\cos \left[\frac{\pi}{2} \eta_{c}\right]\right)
$$

which holds only for values of $\eta_{\mathrm{c}}$ between 0 and 2 inclusive. Formally, analytical solutions for the radial distribution of the axial velocity fall out of either a Prandtl mixing length hypothesis or from an eddy viscosity model attributed to Boussinesq (Bird et al. 2002), Prandtl (Abramovich 1963), and Goertler (Abramovich 1963; Rajaratnam 1976). The Prandtl mixing length hypothesis leaves no simple analytical solution but requires a power series solution given by Tollmien and tabulated in columns 2 and 4 of Table 2-1 in Rajaratnam (1976), shown graphically in Figure 2.4 and approximated as

$$
f_{c}\left(\eta_{c}\right)=0.00940 \eta_{c}^{5}-0.09859 \eta_{c}{ }^{4}+0.39979 \eta_{c}{ }^{3}-0.60105 \eta_{c}{ }^{2}-0.22051 \eta_{c}+1.00394
$$

The eddy viscosity model gives several solutions, perhaps the most convenient of which is

$$
f_{c}\left(\eta_{c}\right)=\left(1+a \eta_{c}^{2}\right)^{-2}
$$

where $a=2^{1 / 2}-1$ so that this function equals $1 / 2$ when $\eta_{\mathrm{c}}=1$ (Bird et al. 2002; Bajpai and Tirumkudulu 2008). ${ }^{1}$ Rajaratnam (1976) and Bird et al. (2002) argue that the eddy viscosity model does marginally better near the center of the jet but worse than the mixing length hypothesis further way from the jet centerline, hence the preference for the latter herein. Figure 2.4a for water suggests that the Tollmien solution may do marginally better for the data presented both in the center and at the largest radial positions measured. However, all represent reasonable fits of the central upwell data, and none of the

\footnotetext{
${ }^{1}$ This solution is termed a Goertler solution in Figure 2.4 for historical reasons following the nomenclature of Rajaratnam 1976, though the form of Eq. 2.8 was first reported by Loytsianskiy (Abramovich 1963) and has been renormalized here.
} 
expressions does markedly better than any other, so each may be used herein where most convenient and mathematically consistent.
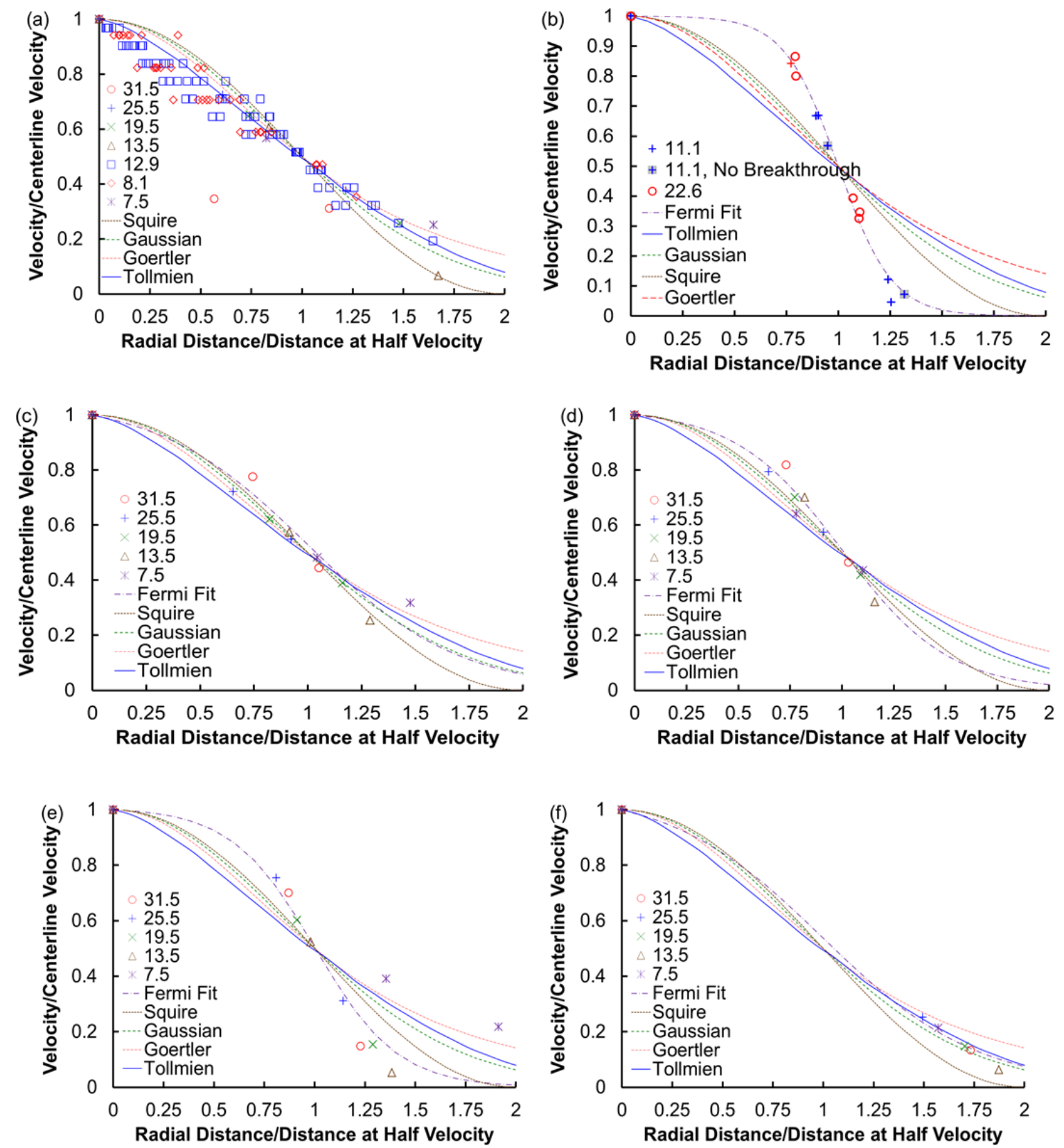

Figure 2.4. Self-similar Velocity Profiles in the Central Upwell with Velocity Scaled on the Centerline Velocity and Radius Scaled on the Radius Corresponding to Half the Velocity for (a) Water, (b) KB Slurry, (c) $10 \mu \mathrm{m}$ Glass Beads at $5 \mathrm{wt} \%$, (d) $10 \mu \mathrm{m}$ Glass Beads at $20 \mathrm{wt} \%$, (e) $35 \mu \mathrm{m}$ Glass Beads at $5 \mathrm{wt} \%$, and (f) $35 \mu \mathrm{m}$ Glass Beads at $20 \mathrm{wt} \%$. Numbers in legends denote elevation scaled on nozzle diameter. Curve fits include the predicted profiles of a Gaussian (Meacham et al. 2012), Squire and Trouncer (Rajaratnam 1976), Goertler (Abramovich 1963; Rajaratnam 1976), and Tollmien (Rajaratnam 1976) as described in the text. Fits with the single parameter normalized Fermi-Dirac function, Eq. (2.9), take $s_{f}=0.368$ (not shown), 0.130, 0.351, $0.259,0.206$, and 0.385 in panels a through $\mathrm{f}$, respectively. 
Radial profiles in the presence of glass beads are shown in Figure 2.4c through Figure $2.4 \mathrm{f}$ as extracted from Bontha et al. (2003b). They measured the velocity profiles along the centerline and at 17 and 24 inches from the jet/vessel centerline using Valeport probes at five elevations affixed to a vertical mast. ${ }^{1}$ The average velocities of all electronic data were tabulated for all reliable pulse cycles associated with tests using water (Table 4.1, Bontha et al. 2003b), $10 \mu \mathrm{m}$ glass beads at $5 \mathrm{wt} \%$ (Table 5.2, Bontha et al. 2003b), $10 \mu \mathrm{m}$ glass beads at $20 \mathrm{wt} \%$ (Table 5.5, Bontha et al. 2003b), $35 \mu \mathrm{m}$ glass beads at $5 \mathrm{wt} \%$ (Table 5.8, Bontha et al. 2003b), and $35 \mu \mathrm{m}$ glass beads at $20 \mathrm{wt} \%$ (Table 5.11, Bontha et al. 2003b). The beads had a specific gravity of 2.49 (Section 5.1, Bontha et al. 2003b; size distributions at various locations given in Appendix B, Bontha et al. 2003b).

Each of these panels (Figure 2.4c through Figure 2.4f) clearly shows overlap of the scaled radial distributions across the elevations, confirming the self-similarity of the profile except perhaps at the lowest elevation that may lie in the entrance region. Panels with simulant appear to have a marginally steeper slope than expected, suggesting that the particles may enhance the velocities closer to the jet centerline. The larger particle sizes or higher concentrations tend to show more steepening. ${ }^{2}$ This is consistent with the findings of Melville and Bray (1979a, b), Mostafa and Mongia (1988), and Barlow and Morrison (1990), who show that increasing the particle density or size increases the local velocity and that the particles tend to concentrate toward the center of particle-laden jets relative to the fluid velocity profile and the entrained fluid at the periphery of the jet remains more dilute (see Section 4.2). To facilitate analysis in Section 3, a best fit curve was prepared with a normalized Fermi-Dirac function

$$
f_{c}\left(\eta_{c}\right)=\frac{1+\operatorname{Exp}\left[-1 / s_{f}\right]}{1+\operatorname{Exp}\left[\left(\eta_{c}-1\right) / s_{f}\right]}
$$

where $s_{f}$ is the single fitting parameter. The fits are also shown in Figure 2.4.

Figure $2.4 \mathrm{~b}$ shows the velocity profiles for KB slurry obtained from Bamberger et al. (2005). They measured velocities 10.5 inches away from a center 15.6 inches to the west of the centerline of the $12.75 \mathrm{ft}$ vessel along each of the four cardinal directions. Although this center may coincide with the approximate jet center as expressed at the fluid surface due to persistent superficial crossflow at the top of this vessel (see Figure 4.5, Bontha et al. 2003b), at lower elevations the east-most probe consistently measures higher velocities, which comports with the jet centerline remaining closer to the vessel centerline. Taking the vessel center as the jet center and linearly fitting the west probe velocity and average of north and south probes to find the distance corresponding to half the velocity of the east probe yields Figure 2.4b. The velocity profiles remain self-similar for the available data regardless of breakthrough (see Appendix A for definition), though data near the jet penetration front are not available.

\footnotetext{
${ }^{1}$ The vertical mast may marginally affect the flow by providing a shear surface. Figure 3.2 of Bontha et al. (2003b) suggests that the uncertainty in probe position from the centerline is $\sim 0.5$ inches. Conversely, the centerline mast is a 0.75 inch pipe (Figure 3.2, Bontha et al. 2003b) analogous to a sparger, suggesting that spargers may not significantly adversely affect local flow profiles.

${ }^{2}$ Radial velocity profiles cannot be ascertained from testing in the $8 \mathrm{ft}$ vessel at $\leq 12 \mathrm{~m} / \mathrm{s}$. Probes were only located near the vessel centerline at the walls for all Newtonian simulants in run sheets 1,2,6, and 7 . Therefore, how the radial velocity profiles of the central upwell were affected by increasing particle size remains unknown. A middle velocity probe was included for testing in the $8 \mathrm{ft}$ test vessel 18.9 inches from center for run sheets 3 through 5 for the KB slurry. However, at velocities $\leq 12 \mathrm{~m} / \mathrm{s}$, no net upward flow was registered by this probe after the initial vortex passed. The vortex was found at $12 \mathrm{~m} / \mathrm{s}$ but not at $8 \mathrm{~m} / \mathrm{s}$, as discussed in Section 9 .
} 
However, this panel shows that the radial velocity profiles for the KB slurry do not follow the Tollmien solution or similar radial velocity profiles derived for homogeneous fluids without a yield stress. Indeed, the velocity profile falls off precipitously as a function of radial position, similar to a plug flow approximation discussed in Section 6.3. The physics underlying these profiles and their implications is discussed in Section 6.

The radial location at half the centerline velocity, $\delta_{c}$, is the characteristic width of the jet. Figure 2.5 shows how this characteristic dimension, determined as described from Eq. (2.4), varies as a function of elevation, where both are scaled on nozzle diameter. Where the fluid contains particles, larger particle concentrations lead to a broader base but a narrower spread, whereas lower particle concentrations have a narrow base but a larger spread (Figure 2.5). The jet appears to narrow with larger particle size at all elevations, though how this trend would continue with increasing particle size remains unknown. ${ }^{1}$ Similarly, the KB slurry jet increases in diameter as a function of elevation, but this increase remains the slowest for all conditions for which data are available. Though the radial increase is marginal, it is significant (the error bars shown are very small) and supports an expanding jet flow regime when the jet achieves breakthrough, which in turn implies a contraction at least sometimes when the jet fails to break through.

\footnotetext{
${ }^{1}$ There is a compelling counter-argument that the yield stresses may be a model artifact. Yield stresses may arise from particle jamming for larger particles (Man et al. 2005) or from interparticle repulsive forces for smaller particles (Larson 1999, 6.4.2; Russel et al. 1989). (That repulse forces instead of attractive forces are the source of colloidal yield stresses and hindered settling [Russel et al. 1989, Chapters 12 and 14] suggests that the term "cohesive" solids may be a convenient but inaccurate misnomer.) For hard spheres, Larson (1999, Chapter 6.2) argues that although the relative viscosity may increase at low shear stresses, it plateaus so that a yield stress does not develop, though extrapolations at larger shear stresses would have suggested a yield stress. Alternatively, for electrostatically charged colloidal particles, Russel et al. (1989, Chapter 14) argue that as the ionic strength decreases, the relative viscosity may increase by orders of magnitude and the particles may undergo an order-disorder transition. Without such a transition, the particles may be simply a disordered, high-viscosity fluid like a slowly flowing glass. However, neither the charge distributions of the waste feed nor the viscosities of actual waste at sufficient low shear stress have been reported (Wells et al. 2011; Gauglitz et al. 2010), and as such it remains unclear whether the yield stress arises from a locally ordered solid state or is simply a fitting artifact. (Simulants have only been explored preliminarily and remain inconclusive with respect to the larger question [Chun et al. 2010].) If the yield stress is artificial, then the "cavern" height limits given Section 6 do not govern, the $12 \mathrm{~m} / \mathrm{s}$-is-12 m/s arguments do not necessarily hold regardless of apparent yield stresses, and momentum conservation must include as yet unmeasured buoyancy considerations in the presence of significant interparticle forces. Moreover, the mixing may be much more substantial than projected in Section 9. However, because the WTP design process assumes a yield stress, in the absence of conclusive evidence either way, this report considers the influence of a putative yield stress and addresses the physical implications thereof.
} 


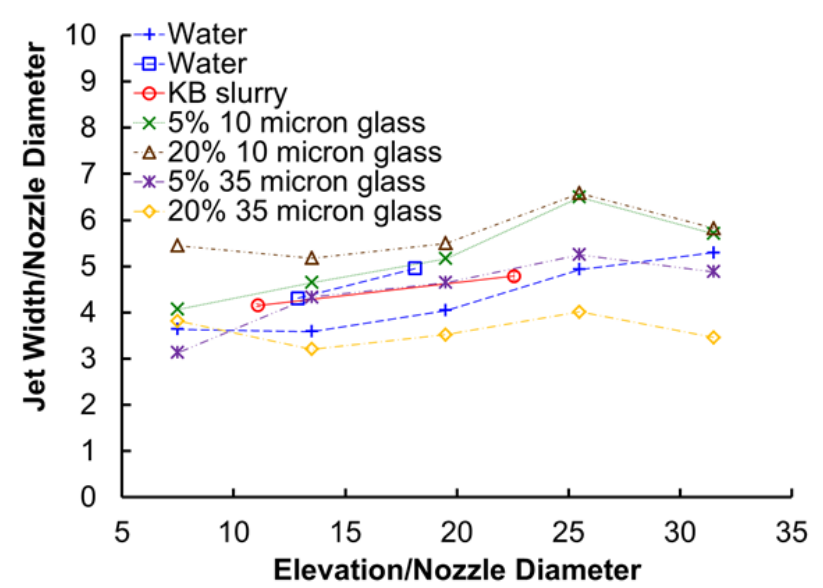

Figure 2.5. Jet Width $\delta_{\mathrm{c}}$ Scaled on Nozzle Diameter as a Function of Scaled Elevation for Water (Bontha et al. 2003a, b), KB Slurry (Bamberger et al. 2005), and Glass Beads of Size and Concentration Indicated (Bontha et al. 2003b). Error bars shown for KB slurry (inside red circles).

Table 2.1 summarizes the trends in $\beta_{c}$, the fractional width at the base of the upwell, $f_{u}$, the virtual origin scaled on the nozzle diameter, and $z_{o} / d_{o}$, in the region of $z / d_{o}$ from 10 to 30 where entrance effects and superficial surface crossflows do not influence the data significantly. These values were determined by asserting the jet to be a right cylindrical cone of radius $\delta_{c}$ through its full traverse from the virtual origin upward (to the right in the plot) in the $12.75 \mathrm{ft}$ vessel. The water curves give values of $\beta_{c}$ of $0.112-0.123$ (see Table 2.1) in approximate agreement with the literature values of $\beta_{c}$ of approximately 0.065 to 0.14 (see Rajaratnam 1976; Bird et al. 2002; Bajpai and Tirumkudulu 2008; Crapper and Baines 1976; Shekarriz et al. 1996). Notably, the fractional width is approximately $10 \%$ to $20 \%$ of the vessel radius but may vary between vessel sizes. This value is used in Section 3. Though the radial distribution of velocities that informs these values is essential to evaluating flow within mixing vessels, radial distributions of velocity and density have not been collected at other scales.

The scaled virtual origin also varies substantially, from 15 to 64 . The range of variation is unexpected because straightforward models of the jet would have anticipated that the virtual origin could be approximated by the total distance the jet travels from the jet nozzle to the upwell (central or wall). For example, "cavern" height models anticipate that the distance from the central upwell may be approximated by the sum of the nozzle stand-off distance from the vessel bottom and the distance along the vessel bottom to the upwell (Poloski et al. 2004; Bamberger et al. 2005; Meyer et al. 2006; Meyer and Etchells 2007; Gauglitz et al. 2009). For the $12.75 \mathrm{ft}$ vessel, the 4 inch nozzles stood off the bottom 6-9 inches and were located $\sim 95$ inches across the center from each other (Figure 2.8, Bontha et al. $2003 \mathrm{~b}$ ). This leads to a suggested travel distance of only $z_{o} / d_{o}=13$, which remains at the lower end of values determined across the set of simulants but is much smaller than the value of 64.3 determined experimentally for the KB slurry. Nevertheless, this variation in $z_{o} / d_{o}$ may explain the variation in the intercept of the "cavern" height models and motivates the effort described in Sections 4 and 6 to understand jets traversing non-Newtonian materials.

It is noted that the data in the radial direction remains limited with measurements of at most three radial locations and scaled elevations to $z / d_{o} \leq 35$. Each data set was obtained in a vessel with a 2:1 elliptical head, yet how jet spread may be affected by the shape of the vessel bottom has not been 
explored. Nevertheless, the data analyzed here support the argument that the central upwell velocity profiles are self-similar and follow jet characteristics within the domain explored.

Table 2.1. Values Defining Central Jet Cone from Figure 2.5 for $10<z / d_{o}<30$

\begin{tabular}{|c|c|c|c|c|}
\hline Reference & Fluid & $\beta_{c}$ & $f_{u}$ & $z_{o} / d_{c}$ \\
\hline Bontha et al. (2003a) & Water & 0.123 & 0.142 & 22.1 \\
\hline Bontha et al. (2003b) & Water & 0.112 & 0.105 & 17.9 \\
\hline Bamberger et al. (2005) & KB slurry & 0.0551 & 0.185 & 64.3 \\
\hline Bontha et al. (2003b) & $5 \% 10 \mu \mathrm{m}$ glass beads & 0.154 & 0.128 & 15.9 \\
\hline Bontha et al. (2003b) & $20 \% 10 \mu \mathrm{m}$ glass beads & 0.117 & 0.181 & 29.6 \\
\hline Bontha et al. (2003b) & $5 \% 35 \mu \mathrm{m}$ glass beads & 0.0763 & 0.170 & 42.7 \\
\hline Bontha et al. $(2003 b)^{(a)}$ & $20 \% 35 \mu \mathrm{m}$ glass beads & 0.0676 & 0.118 & 33.4 \\
\hline
\end{tabular}

(a) Based only the centerline and one radial position whereas the remaining Bontha et al. (2003b) results are based on the centerline and two radial positions.

\subsection{Attributes of 8-Foot Vessel Testing}

Velocity data from the $8 \mathrm{ft}$ test vessel have also been analyzed. Table 2.2 gives the physical properties of simulants used in these tests, including mass or weight fraction of species $i, w_{i}$, median particle size, ${ }^{1} d_{50}$, terminal settling velocity $u_{i}{ }^{T}$, and Archimedes number, $\mathrm{Ar}_{i}$, given as

$$
\operatorname{Ar}_{i}=\rho_{f}^{2} g\left(s_{i}-1\right) d_{50}{ }^{3} / \mu_{f}^{2}
$$

where $\mu_{f}$ and $\rho_{f}$ are the dynamic viscosity and density of the fluid surrounding the particle of interest, $g$ is the gravitational constant, and $s_{i}$ is the ratio of the solids density to the fluid density of species $i$. For a Newtonian slurry, Wells et al. (2012) find preferred agreement of Hanford wastes with the model of Camenen (2007) as

$$
u_{i}^{T}=\frac{\mu_{f}}{\rho_{f} d_{50}}\left[\left(15+\left(\frac{\mathrm{Ar}_{i}}{0.3}\right)^{0.5}\right)^{0.5}-15^{0.5}\right]^{2}
$$

\footnotetext{
${ }^{1}$ The median particle size has been retained here, though Peurrung et al. (2015) suggest a variety of other particle size and velocity metrics that may be used in its place. Russel et al. (1989) indicate that settling properties depend strongly on interparticle forces and potentials, which have not been measured for Hanford wastes but may be the source of the proliferation of some of these metrics.
} 
This model is essentially a hard sphere model that neglects interparticle repulsive or attractive forces in an otherwise Newtonian fluid (Russel et al. 1989). Figure 2.6 shows the shear stress versus the shear rate for the KB slurries used in these tests. ${ }^{1}$

Table 2.2. Ambient Physical Properties of Simulants Used in the $8 \mathrm{ft}$ Test Vessel

\begin{tabular}{lcccccccc}
\hline \multicolumn{1}{c}{ Constituent } & $\begin{array}{c}\text { Run } \\
\text { Sheet }\end{array}$ & Order & $\begin{array}{c}\text { Particle } \\
\text { Density } \\
\left(\mathrm{g} / \mathrm{cm}^{3}\right)\end{array}$ & $\begin{array}{c}w_{i} \\
(\text { solids } \\
\text { only) }\end{array}$ & $\begin{array}{c}w_{i} \\
\text { (in } \\
\text { suspension) }\end{array}$ & $\begin{array}{c}d_{50} \\
(\mu \mathrm{m})\end{array}$ & $\mathrm{Ar}$ & $\begin{array}{c}u_{i}{ }^{T} \\
(\mathrm{~m} / \mathrm{s})\end{array}$ \\
\hline Glass powder & $1-2^{(\mathrm{a})}$ & - & 2.5 & 0.900 & 0.027 & 96 & 13 & $6.2 \cdot 10^{-3}$ \\
$\mathrm{Al}_{2} \mathrm{O}_{3}$ & $1-2^{(\mathrm{a})}$ & - & 3.9 & 0.100 & 0.003 & 350 & $1.2 \cdot 10^{3}$ & $7.2 \cdot 10^{-2}$ \\
$\mathrm{~KB}^{2}$ slurry & $3^{(\mathrm{b})}$ & - & 2.6 & $0.80: 0.20$ & 0.3234 & $\sim 5$ & - & - \\
$\mathrm{SiC}$ & $7^{(\mathrm{c})}$ & 3 & 3.2 & 0.520 & 0.0628 & 5 & $2.7 \cdot 10^{-3}$ & $3.0 \cdot 10^{-5}$ \\
$\mathrm{~W}^{(\mathrm{d})}$ & $7^{(\mathrm{c})}$ & 2 & 9.6 & 0.040 & 0.0048 & 16 & $3.5 \cdot 10^{-1}$ & $1.2 \cdot 10^{-3}$ \\
$\mathrm{Glass} \mathrm{powder}^{(\mathrm{c})}$ & $7^{\text {c }}$ & 1 & 2.5 & 0.380 & 0.0459 & 103 & 16 & $7.0 \cdot 10^{-3}$ \\
$\mathrm{Al}_{2} \mathrm{O}_{3}$ & $7^{(\mathrm{c})}$ & 5 & 3.9 & 0.035 & 0.0042 & 432 & $2.3 \cdot 10^{3}$ & $9.0 \cdot 10^{-2}$ \\
\hline Sand & $7^{(\mathrm{c})}$ & 5 & 2.65 & 0.010 & 0.0012 & 876 & $1.1 \cdot 10^{4}$ & $1.3 \cdot 10^{-1}$ \\
Glass beads & $7^{(\mathrm{c})}$ & 4 & 2.5 & 0.015 & 0.0018 & 1080 & $1.9 \cdot 10^{4}$ & $1.4 \cdot 10^{-1}$
\end{tabular}

(a) Settling velocities from run sheet 1, CCN 270902; confirmed with Eq. (2.11). Ar from Eq. (2.10). FIO.

(b) Personal communication from Carolyne Burns of PNNL. Ar and $u_{i}^{T}$ from Eqs. (2.10) and (2.11). FIO.

(c) From Tables 1 and 2 of Herting et al. (2012) and from M. Minette "MCE Loni testing through 10.doc" on $3 / 19 / 2015$ with order of addition and suspension weight fractions based on $12.08 \%$. FIO.

(d) $\mathrm{W}$ denotes the dense tungsten component, tungsten carbide with a density of $9.6 \mathrm{~g} / \mathrm{cm}^{3}$.

\footnotetext{
${ }^{1}$ From CCN 272779, pp. A14-A15. The hysteresis in these curves remains negligible with data collected across weeks, so that physical or chemical gelation processes will not be considered further herein. However, hysteresis and temporal transience have been significant in prior simulants, and gelation rates have been estimated in WTP simulants (Poloski et al. 2003, 2004; Powell et al. 1997). Where this is significant, it may be analyzed from irreversible thermodynamics principles, recognizing that cavern formation, for example, may be simply a two-phase phenomenon with fluid and solid phases. Eq. 2-32 in Rajaratnam (1976) shows that turbulence production in the vessel is associated with entropy production and may be mapped out spatially with velocity profiles developed herein. Turbulence production is equivalent to power per unit volume as shown by Kuhn et al. (2013). Russel et al. (1989) and Larson (1999) provide expressions for and from which colloidal enthalpy and aggregation rates may be expressed that may vary spatially, with even modest stratification in composition. Gordon (2003) describes how to generate the relevant phase diagrams that can then be mapped out spatially. Combined, this analysis may establish the time-average "cavern" map from first principles using rheology and particle size, density, and charge measurements directly.
} 


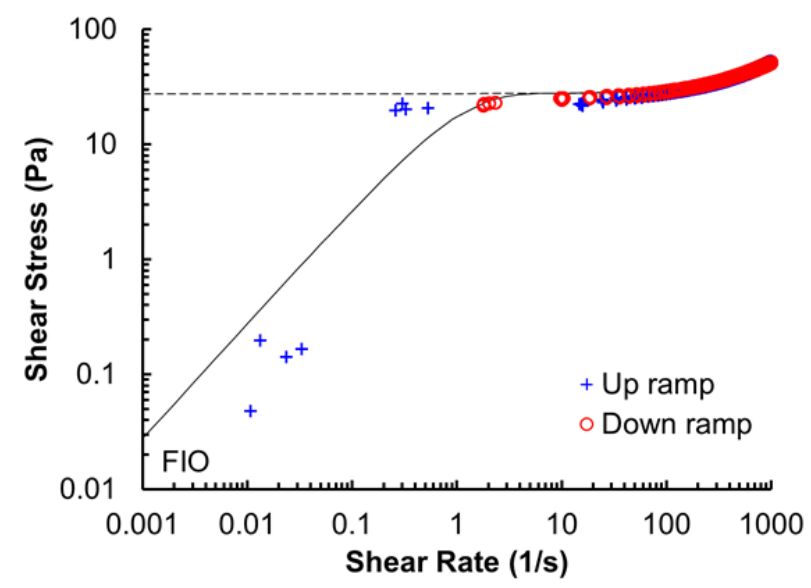

Figure 2.6. Shear Stress versus Shear Rate for Up Ramp (blue +) and Down Ramp (red circle) for KB Slurry Used in Run Sheets 3 and 4. This shows that creep flow may set in at shear rates below $\sim 0.3 \mathrm{~s}^{-1}$. The characteristic curves are (solid) for the Bingham-Papanastasiou model $\left(\tau=\tau_{o}[1-\exp (-m \dot{\gamma})]+\mu_{o} \dot{\gamma}\right.$ with a consistency of $\mu_{o}=25.75 \mathrm{mPa}$ s and the yield stress is $\tau_{o}=27.58 \mathrm{~Pa}$, and $m(\sim 1 \mathrm{~s})$ is a regularization parameter with dimensions of time [Mitsoulis 2007]) and (dashed) the ideal Bingham plastic $(m \rightarrow \infty)$. The former allows for determination of yielded/unyielded regions a posteriori and facilitates theoretical analysis in Section 4. For information only (FIO). Data courtesy of Carolyne Burns of PNNL.

Central upwell data with three velocity probes located along the vessel centerline were collected for a two-part Newtonian simulant composed of alumina and glass beads in run sheets 1 and 2, for a KB slurry in run sheet 3, and for multipart Newtonian simulants in run sheet 7. See Figure 2.7. The velocity probes were moved to near the vessel wall in run sheets 4 through 5 . 

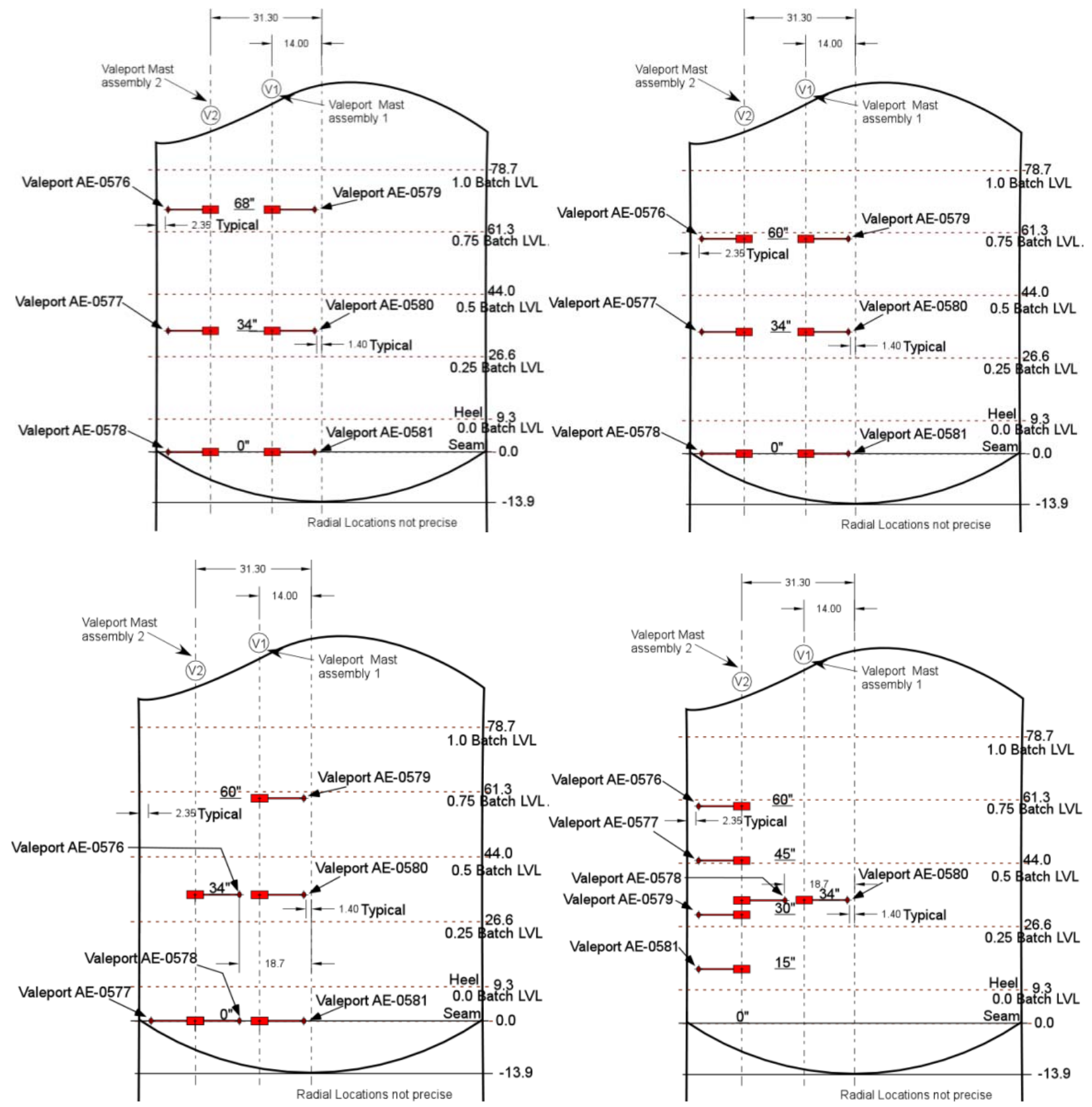

Figure 2.7. Positions of Valeport Velocity Probes for Run Sheets (a) 1; (b) 2, 6, and 7; (c) 3; and (d) 4 and 5. Courtesy of Korey Peterson and Christopher Chapman. Lateral/radial probe positions not verified. FIO.

To put the analysis that follows in perspective, characteristic curves are considered below for which surface flow was and was not achieved. For example, Figure 2.8 shows a Newtonian simulant for which surface flow was achieved. Figure 2.8a shows that within a fraction of a second after the pressure pulse begins, the level in the PJM tube begins to drop and the radar level measurement begins to rise. The level drop continues linearly until the pressure is turned off, then the levels in the PJM tube and the vessel reach their minimum and maximum levels, respectively. These levels then gradually and smoothly return to their initial values. Approximately $2.5 \mathrm{~s}$ after the start of the pulse (see Figure $2.8 \mathrm{~b}$ ), a velocity is registered at the vessel centerline at the seam. (The velocity data includes a $2.25 \mathrm{~s}$ delay due to a signal 
processing constraint not adjusted herein.) The steepest slope (a measure of the penetration front of the jet similar to the thresholding of Bajpai and Tirumkudulu 2008) is registered at the seam $3.9 \mathrm{~s}$ after the pulse begins, $5.1 \mathrm{~s}$ at 34 inches above the seam, and $6.5 \mathrm{~s}$ at 68 inches above the seam. These times represent the transience or celerity of the jet; celerity means rapidity or swiftness of motion. As the jet reaches the highest probe, both azimuthal (hoop) and vertical velocities were registered. This synchronous measurement of the velocities in both directions is commensurate with the passage of a turbulent leading vortex.

Figure 2.9 depicts the structure of the leading vortex at the jet penetration front. Kuhn et al. (2013) argue that flow rate within the core of the jet exceeds the penetration rate of the jet front. This difference in flow rates causes the fluid to diverge radially outward, which gives rise to the leading vortex.

Turbulence associated with this vortex gives rise to transient azimuthal velocities. Therefore, an essential feature of a leading vortex is transverse flow near the inflection point in the axial velocity profile. Indeed, a spike in the azimuthal flow profile is observed at the inflection point in the axial velocity profile as expected. The magnitude of instantaneous velocity variations about the average velocity after passage of the leading vortex suggests that the turbulence remains strong even at the highest probe. Also worth noting is the gradual increase in the axial velocity at the top probe before the first vortex passes, indicative of bulk level rise in the vessel before the first pulse arrives. The magnitude of this velocity is on the order of tens of millimeters per second for bulk fluid motion. 

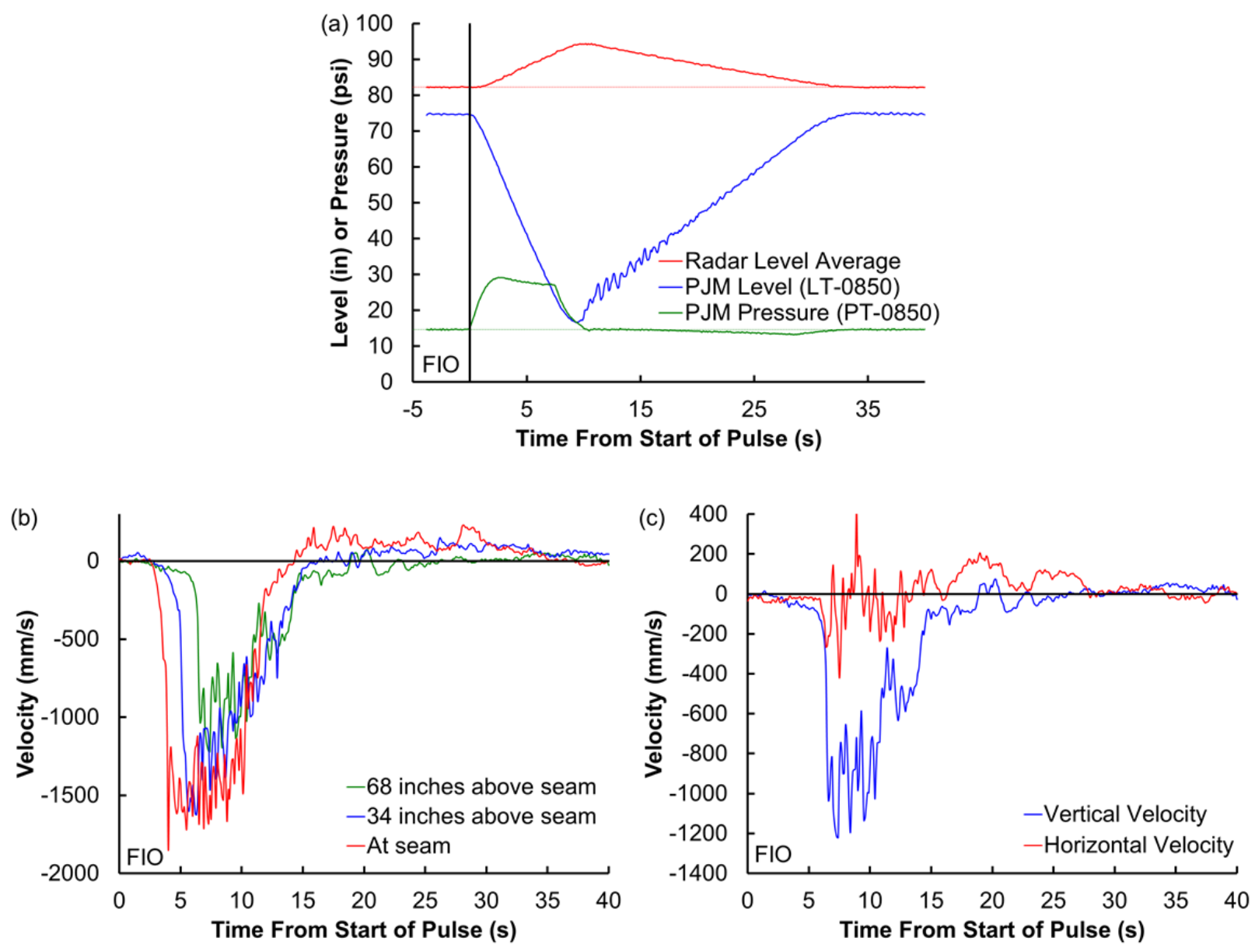

Figure 2.8. Exemplary Characteristics of Test 1-8.4.3 (time stamp 075218): (a) PJM Level, PJM Pressure, and Radar Level Average; (b) Vertical Velocities 0, 34, and 68 inches Above the Seam; and (c) Vertical and Horizontal Velocities at 68 inches Above Seam Versus Time from the Start of the Pulse. This test was conducted in the $8 \mathrm{ft}$ vessel with $H / D=1.0$, $d_{o}=1.94$ in, $U_{o}=12 \mathrm{~m} / \mathrm{s}$, pulse volume fraction $(\mathrm{PVF})=0.1$ using the $\mathrm{S}$ firing sequence with the two-part Newtonian simulant. Surface flow reported as "sustained roll-over of entire field of view" (Bontha et al. 2015, Appendix G). FIO. 


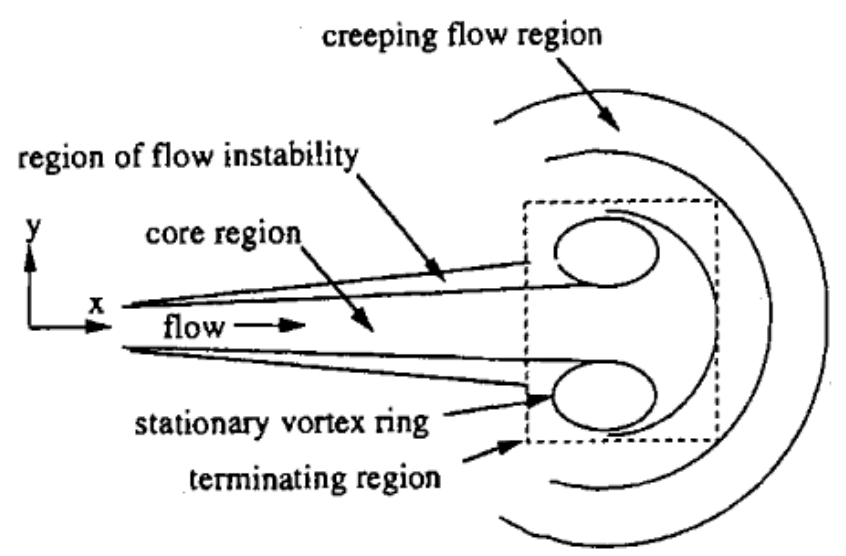

Figure 2.9. The Leading Penetration Front of a Jet Showing the Jet Core and Leading Vortex Ring (Shekarriz et al. 1995b). The leading vortex may be stationary. Original caption: "Fig. 4 Schematic diagram showing the various flow regions within the pseudoplastic jet." Used with permission from ASME.

Figure 2.10 shows similar characteristics for test 1-8.4.2, which differs only in that the nominal velocity is $9.4 \mathrm{~m} / \mathrm{s}$ and surface flow was not achieved. This figure shows again a smooth rise and fall of the levels and pressures similar to Figure 2.8. The vertical velocities rise as before with maximum slopes at 4.6 and $6.2 \mathrm{~s}$ at the seam and 34 inches above the seam, respectively. There are strong suggestions of a leading vortex at 34 inches with both horizontal and vertical motion. However, here a cloud height was observed at 62 inches and flow characteristic of a turbulent jet never arrives at the upper velocity transducer 68 inches above the seam. At the top transducer there is a steady initial rise suggestive of the bulk level rise, albeit smaller as expected for a longer drive time. Undulations around the time-averaged velocity are small and smooth, suggesting that the turbulence has all but vanished. Low frequency oscillations akin to the coherent motion reported by Kuhn et al. (2013) in Meyer et al. (2012) data may be observed. However, there is no indication of a vortex or turbulence 68 inches above the seam. 

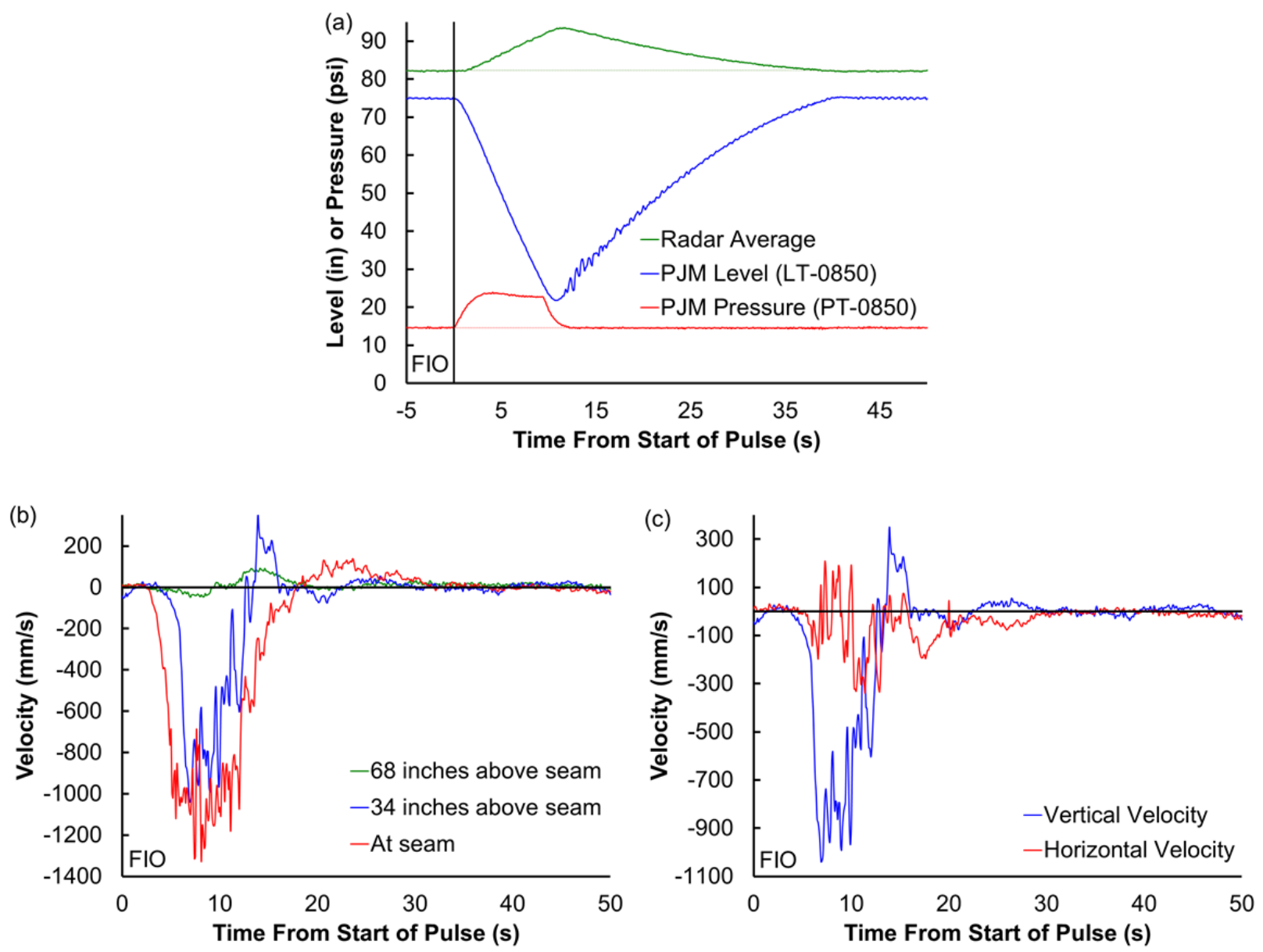

Figure 2.10. Exemplary Characteristics of Test 1-8.4.2 (time stamp 052539): (a) PJM Level, PJM Pressure, and Radar Level Average; (b) Vertical Velocities 0, 34, and 68 inches Above the Seam; and (c) Vertical and Horizontal Velocities at 34 inches Above Seam Versus Time from the Start of the Pulse. This test was conducted in the $8 \mathrm{ft}$ vessel with $H / D=1.0$, $d_{o}=1.94$ inches, $U_{o}=9.4 \mathrm{~m} / \mathrm{s}, \mathrm{PVF}=0.1$ using the $\mathrm{S}$ firing sequence with the two-part Newtonian simulant. Surface flow reported as "not near surface" (Bontha et al. 2015, Appendix G). FIO.

Similar insight is obtained for the processes occurring along the vessel centerline for the non-Newtonian KB slurry of run sheet 3 . Figure 2.11 shows an initial sigmoidal increase in velocity that occurs simultaneously throughout the vertical extent of the vessel, both along the vessel centerline and along the wall (data not shown). This is consistent with rigid solid motion because all four of these probes register a change in velocity of the same magnitude at the same time. This suggests a time scale for release of the bulk solid of $1.8 \pm 0.1 \mathrm{~s}$ (range). The rate of level change in the PJM tubes (i.e., the change in slopes) occurs at $2.0 \pm 0.3 \mathrm{~s}$, nearly simultaneous with the initial sigmoidal increase in the velocity. The magnitude of the slope in the PJM level drop decreases thereafter. This change in slope occurs at the same time that the surface level begins to rise. All of the non-Newtonian files analyzed (see Table 2.3) have this initial sigmoidal increase in velocity, suggesting that this is a systematic trend instead of a small test anomaly. This sigmoidal velocity increase or double slope in the level is not present in data files for any of the other simulants analyzed. Once motion begins, the speed associated with bulk rigid motion is approximately 45 to $75 \mathrm{~mm} / \mathrm{s}$ (see Section 3.3). Because bulk motion appears to be free of 
acceleration after an induction period from $\sim 1.8-2.5 \mathrm{~s}$ in test $3-8.5 .1$, bulk motion may be subtracted kinematically from the apparent jet velocity to arrive at the actual jet velocity if desired (see Section 3.3).

As the pulse reaches up into the central bulk of the fluid, the front end of the pulse does not seem to slow with a square root or fourth root dependence suggested for jets or puffs, respectively (Kuhn et al. 2013; Bajpai and Tirumkudulu 2008; Sangras and Faeth 1999). Again, velocities are registered both vertically and azimuthally, characteristic of the leading vortex. Thereafter several vortices follow. The width (i.e., frequency) and magnitude of these pulses is an indication of the turbulence. At higher elevations, both change. The frequency seems to decrease in nearly all of the velocity measurements with higher elevation, indicative of damping of higher frequency vortices, but the magnitude of the turbulence may or may not dampen.

At the tail end of the passing jet, the velocity crosses through zero at approximately the same time for all elevations. Less temporal spread is observed at the back end of the pulse than at the front end. A suction pulse (positive velocity in these plots) is observed at multiple elevations and is typically, though not always, observed at all elevations. This is consistent with the observation that some fraction of the fluid breaking through the surface returns back down the same hole through which it emerged (CCN 272778). These curves suggest that this is not exclusively a surface effect. Suction affects the estimates of the rate of vessel mixing because it implies that subsequent pulses mobilize some of the same fluid repeatedly. 

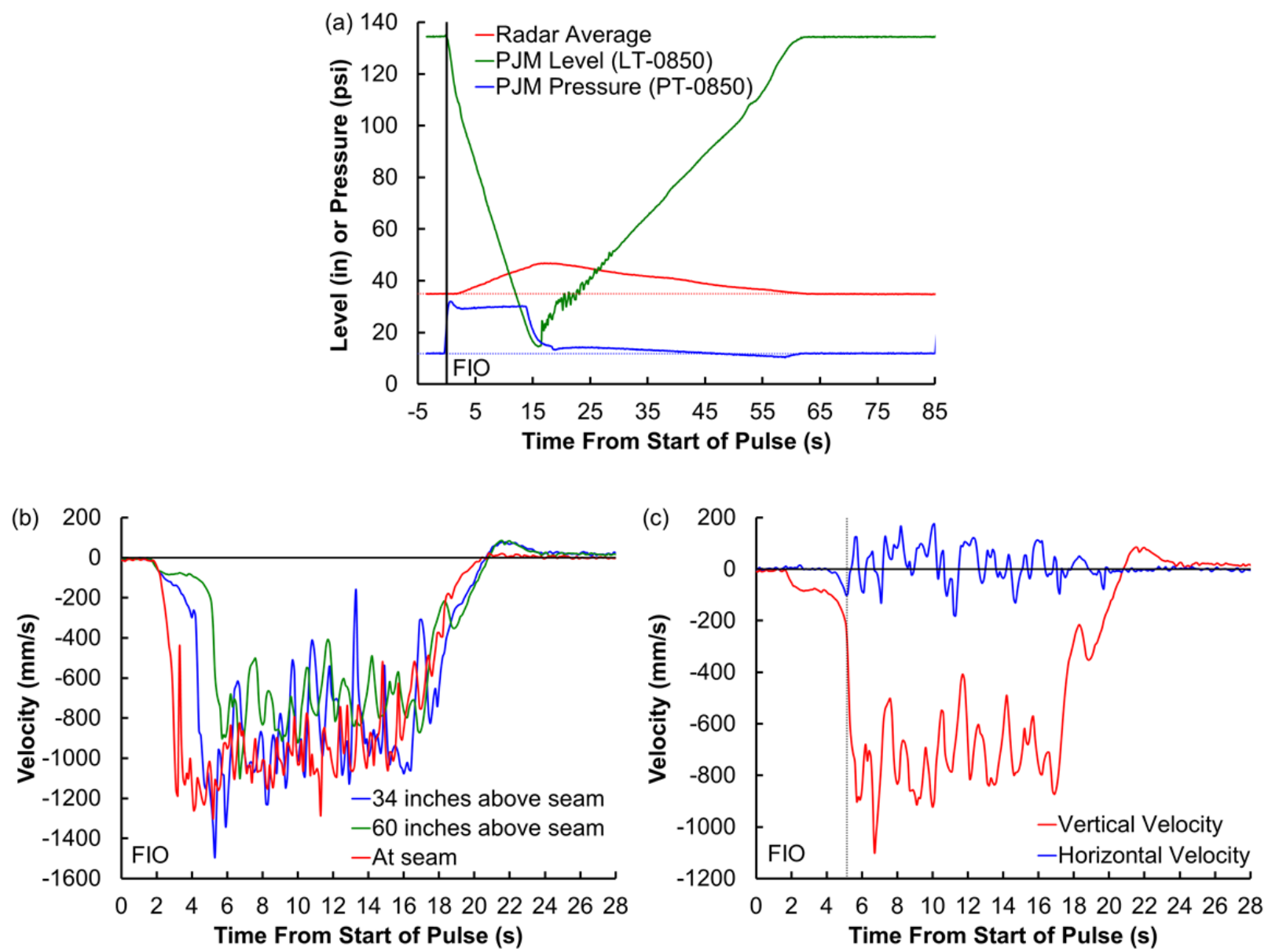

Figure 2.11. Exemplary Characteristics of Test 3-8.5.1 (time stamp 140349): (a) PJM Level, PJM Pressure, and Radar Level Average; (b) Vertical Velocities 0, 34, and 60 inches Above the Seam; and (c) Vertical and Horizontal Velocities at 60 inches Above Seam Versus Time from the Start of the Pulse. This test was conducted in the $8 \mathrm{ft}$ vessel with $H / D=1.0$, $d_{o}=1.94$ inches, $U_{o}=12 \mathrm{~m} / \mathrm{s}, \mathrm{PVF}=0.2$ using the $\mathrm{S}$ firing sequence with the KB slurry. Surface flow reported as "1-Strong" (Bontha et al. 2015, Appendix G). FIO. 

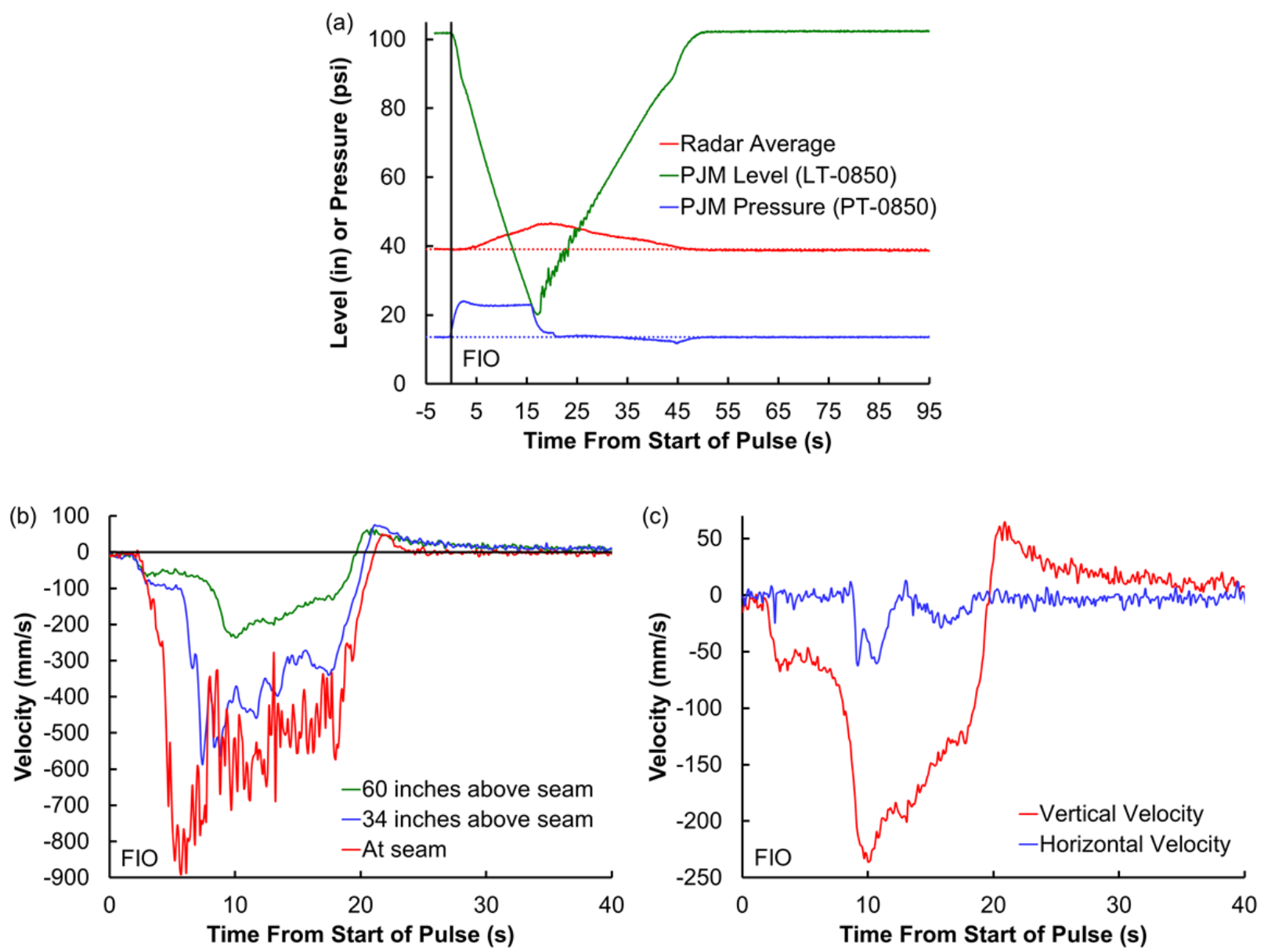

Figure 2.12. Exemplary Characteristics of Test 3-8.1.44 (time stamp 032930): (a) PJM Level, PJM Pressure, and Radar Level Average; (b) Vertical Velocities 0, 34, and 60 inches Above the Seam; and (c) Vertical and Horizontal Velocities at 60 inches Above Seam Versus Time from the Start of the Pulse. This test was conducted in the $8 \mathrm{ft}$ vessel with $H / D=1.0$, $d_{o}=1.94$ inches, $U_{o}=8 \mathrm{~m} / \mathrm{s}, \mathrm{PVF}=0.15$ using the $\mathrm{S}$ firing sequence with the KB slurry. Surface flow reported as "5-NA" (Bontha et al. 2015, Appendix G). FIO.

Figure 2.12 shows the same intriguing behavior along the front of the pulse with two slopes on the PJM level curves. As before, the PJM pressure pulse first peaks and resolves and then the surface registers a rise. The steepest slope occurs at the seam at $4.5 \mathrm{~s}$, at 34 inches at $6.2 \mathrm{~s}$, and at 60 inches at $8.6 \mathrm{~s}$. Again, this suggests a jet front that advances approximately linearly with time instead of the $n^{\text {th }}$ root dependencies suggested elsewhere (Kuhn et al. 2013; Bajpai and Tirumkudulu 2008; Sangras and Faeth 1999).

An important feature of the curves in Figure 2.12 is the fluctuations around the mean velocity. The fluctuations are strong at the seam but dampen considerably in frequency and magnitude with increasing elevation, suggesting that the turbulence of the jet vanishes at higher elevations. Indeed, the jet is fully turbulent at the bottom of the vessel but begins to lose its turbulence by the middle of the vessel with larger vortices preserved, but the smaller vortices have dampened. Near the top probe, only one or two of the largest eddies remain, including the initial vortex, and the flow is all but laminar. By the top of the vessel, no surface flow was reported. The surface for $H / D=1$ ( $H$ is the vessel fill height and $D$ is the 
vessel diameter) would be located at 78.6 inches above the seam so the jet had 18 inches remaining in its rise, but did not arrive based on visual observations. Since the vessel composition is nearly homogeneous and the particle size is small, buoyancy effects would not necessarily be expected to be a dominant mechanism for loss of turbulence, though this report does not quantitatively evaluate or confirm the validity of this claim.

\subsection{Analysis of Centerline Data from the 8 Foot Vessel}

Among the data collected in the $8 \mathrm{ft}$ vessel, each data file contained at least five complete pulses (often many more) from which averages were obtained. The PJMs (labeled 0501 through 0506 arranged clockwise) were operated with S, A1, and A2 firing sequences. In the S sequence, all six PJMs were fired simultaneously. In the A1 sequence, every other PJM was fired and then half of the cycle time later the remaining PJMs were fired. In the A2 firing sequence, every other PJM was fired and at approximately the end of the drive time of the first set, then the remaining PJMs were fired (while the first began to refill). The pulse was identified from the level of PJM 0501 for the S and A1 firing sequences (every other pulse was analyzed for the A1 sequences), or from the combined levels of PJMs 0501 and 0502 for the A2 firing sequence. The beginning of the pulse was determined when the level thereafter dropped consecutively for $0.7 \mathrm{~s}$ (data was collected at $0.1 \mathrm{~s}$ intervals). The end of the pulse was identified as the time when the level (PJM 0501 for S or A1 and PJM 0502 for A2) was lower than or equal to the PJM level for the proceeding and following $0.3 \mathrm{~s}$.

For noisier files, the beginning of the pulse was determined by averaging over $0.3 \mathrm{~s}$ intervals to smooth out the noise and the end of the pulse was determined as the minimum or first of multiple minima of equal depth over $2.1 \mathrm{~s}$. Please note that this pulse definition includes both drive and possibly some portion of the vent processes, though the suction phase of the drive may not yet have started. For each velocity probe, the largest magnitude ${ }^{1}$ was identified and the beginning of velocity averaging proceeded from the first moment in the pulse that the velocity crossed half of the largest magnitude. This estimates approximately (but perhaps not exactly) the inflection point characteristic of the initial vortex passing (Shekarriz et al. 1995b; Kuhn et al. 2013). The end of velocity averaging coincides with the end of the pulse as measured from the level drop. ${ }^{2}$ This definition of velocity may or may not differ from those reported elsewhere. The probe velocities were divided by the actual nozzle velocity as reported by Bontha et al. (2015) or nominal test velocity where the actual nozzle velocity was unavailable to obtain the scaled velocities plotted hereafter. The operating conditions and visual observations of upwell (for Newtonian simulants) and breakthrough (for non-Newtonian simulants) as recorded by the test completion team are summarized in Table 2.3 (Bontha et al. 2015). These tests used the definition of breakthrough from Bontha et al. (2015).

To succinctly characterize upwell and breakthrough in a single number, a surface flow metric is established (see Table 2.3). This metric is

- 1 if (a) any of the following were observed including upwell visually observed, breakthrough observed, surface video shows sustained roll-over of entire field of view or a breakthrough type of

\footnotetext{
${ }^{1}$ Probes were oriented such that the data read a negative value when the velocity was opposed to the gravitational vector (i.e., up). Therefore, the absolute value of all data was taken.

${ }^{2}$ Signal transduction of the Valeport velocity probes delays recording of the velocity information by $2.25 \mathrm{~s}$ relative to the recording of other data such as level and pressure (Bontha et al. 2015, p. 54).
} 
1-Strong or 2-Good (Bontha et al. 2015, Appendix G), and (b) top probe turbulent or turbulent but attenuating unless the top probe was deemed unreliable in which case criteria (a) held independently

- 0 if any of the following hold $\mathrm{N}$ for upwell, $\mathrm{N}$ for brk tru, Not near surface, 5-NA (Bontha et al. 2015, Appendix G)

- 0.5 for all others (e.g., at or near surface, Bontha et al. [2015], Appendix G).

Essentially, the metric is one if turbulent jet flow may be asserted with confidence, zero for no flow at the fluid surface and 0.5 for closer calls. 
Table 2.3. Summary of Test Operating Conditions and Reported Observations for Tests Evaluated ${ }^{(a)}$

\begin{tabular}{|c|c|c|c|c|c|c|c|c|c|}
\hline Test Line & $\begin{array}{c}\text { Time } \\
\text { Stamp }\end{array}$ & Simulant & $\begin{array}{c}U_{o} \\
(\mathrm{~m} / \mathrm{s})^{(\mathrm{b})}\end{array}$ & $\begin{array}{c}d_{o} \\
\text { (inch) }\end{array}$ & PVF & Firing & $\begin{array}{c}\text { Surface } \\
\text { Flow }\end{array}$ & $\begin{array}{c}\text { Cloud } \\
\text { Height }^{(\mathrm{c})}\end{array}$ & $\begin{array}{l}\text { Turbulence at } \\
\text { Top Probe }^{(d)}\end{array}$ \\
\hline Commissioning & 112414 & water & 12 & 1.94 & NR & $\mathrm{S}$ & NR & NR & $\bar{Y}$ \\
\hline $1-8.3 .1$ & 163117 & 2-part & 6.23 & 1.94 & 0.1 & A1 & No & 24 in & $\mathrm{N}$ \\
\hline $1-8.3 .2$ & 204721 & 2-part & 9.40 & 1.94 & 0.1 & A1 & No & 44 in & $\mathrm{N}$ \\
\hline $1-8.3 .3$ & 225515 & 2-part & 11.97 & 1.94 & 0.1 & A1 & Uncertain & 78 in & Pulsy \\
\hline $1-8.4 .1$ & 041232 & 2-part & 6.24 & 1.94 & 0.1 & $\mathrm{~S}$ & No & 26 in & $\mathrm{N}$ \\
\hline $1-8.4 .2$ & 052539 & 2-part & 9.33 & 1.94 & 0.1 & $\mathrm{~S}$ & No & 62 in & $\mathrm{N}$ \\
\hline $1-8.4 .3$ & 075218 & 2-part & 12.03 & 1.94 & 0.1 & $\mathrm{~S}$ & Yes & 79 in & $\mathrm{Y}$ \\
\hline $1-8.5 .1$ & 091231 & 2-part & 11.98 & 1.94 & 0.1 & A2 & Uncertain & 74 in & Unreliable \\
\hline $1-8.5 .2$ & 102908 & 2-part & 9.35 & 1.94 & 0.1 & A2 & No & 45 in & $\mathrm{N}$ \\
\hline $1-8.5 .3$ & 113936 & 2-part & 6.21 & 1.94 & 0.1 & A2 & No & 25 in & $\mathrm{N}$ \\
\hline $1-8.6 .3$ & 204941 & 2-part & 11.94 & 1.94 & 0.2 & A2 & Yes & 79 in & Unreliable \\
\hline $1-8.7 .1$ & 220632 & 2-part & 11.93 & 1.94 & 0.2 & S & Yes & 79 in & Unreliable \\
\hline $1-8.7 .2$ & 001944 & 2-part & 9.41 & 1.94 & 0.2 & $\mathrm{~S}$ & Uncertain & 76 in & Pulsy \\
\hline $1-8.7 .6$ & 081911 & 2-part & 12.01 & 1.94 & 0.1 & $\mathrm{~S}$ & Yes & 79 in & Y \\
\hline $1-8.9 .2$ & 201434 & 2-part & 9.37 & 1.94 & 0.2 & $\mathrm{~S}$ & Uncertain & 73 in & Unreliable \\
\hline $1-8.9 .3$ & 220002 & 2-part & 12.03 & 1.94 & 0.2 & $\mathrm{~S}$ & Yes & 79 in & Unreliable \\
\hline $2-8.3 .1$ & 212533 & 2-part & 7.14 & 2.50 & 0.1 & $\mathrm{~S}$ & No & 47 in & $\mathrm{N}$ \\
\hline $2-8.3 .2$ & 224950 & 2-part & 5.60 & 2.50 & 0.2 & $\mathrm{~S}$ & No & 44 in & $\mathrm{N}$ \\
\hline $2-8.3 .3$ & 004933 & 2-part & 3.69 & 2.50 & 0.2 & $\mathrm{~S}$ & No & 35 in & $\mathrm{N}$ \\
\hline $2-8.3 .4$ & 030446 & 2-part & 7.22 & 2.50 & 0.2 & S & No & 64 in & Pulsy \\
\hline $2-8.3 .5$ & 043608 & 2-part & 9.33 & 2.50 & 0.2 & $\mathrm{~S}$ & Yes & 79 in & Y \\
\hline $2-8.3 .6$ & 081004 & 2-part & 10.54 & 2.50 & 0.2 & S & Yes & 79 in & Y \\
\hline $2-8.5 .1$ & 144130 & 2-part & 3.72 & 2.50 & 0.2 & S & No & 38 in & $\mathrm{N}$ \\
\hline $2-8.5 .2$ & 165007 & 2-part & 5.66 & 2.50 & 0.2 & $\mathrm{~S}$ & No & 53 in & $\mathrm{N}$ \\
\hline $2-8.5 .3$ & 190406 & 2-part & 7.14 & 2.50 & 0.1 & S & No & 55 in & $\mathrm{N}$ \\
\hline $3-8.1 .37$ & 012317 & KB & 8.0 & 1.94 & 0.15 & $\mathrm{~S}$ & No & - & $\mathrm{N}$ \\
\hline $3-8.1 .38$ & 021715 & $\mathrm{~KB}$ & 12.0 & 1.94 & 0.15 & $\mathrm{~S}$ & Yes & - & Attenuating \\
\hline $3-8.1 .44$ & 032930 & KB & 7.9 & 1.94 & 0.15 & S & No & - & $\mathrm{N}$ \\
\hline $3-8.1 .45$ & 042131 & $\mathrm{~KB}$ & 12.1 & 1.94 & 0.15 & S & Yes & - & Y \\
\hline $3-8.3 .1$ & 123732 & KB & 12.0 & 1.94 & 0.2 & A2 & Uncertain & - & Attenuating \\
\hline $3-8.5 .1$ & 140349 & KB & 12.0 & 1.94 & 0.2 & $\mathrm{~S}$ & Yes & - & Attenuating \\
\hline $3-8.58 .3$ & 151941 & $\mathrm{~KB}$ & 15.0 & 1.94 & 0.2 & $\mathrm{~S}$ & Yes & - & Y \\
\hline $3-8.58 .4$ & 160201 & KB & 15.0 & 1.94 & 0.15 & S & Yes & - & Y \\
\hline $7-8.2 .6$ & 071838 & 1-part ${ }^{(\mathrm{e})}$ & 15 & 1.94 & 0.2 & S & Yes & - & Y \\
\hline $7-8.2 .9$ & 122520 & 1-part ${ }^{(\mathrm{e})}$ & 15 & 1.94 & 0.2 & S & Yes & - & Y \\
\hline $7-8.2 .13$ & 071858 & 1-part ${ }^{(\mathrm{e})}$ & 15 & 1.94 & 0.2 & S & Yes & - & Y \\
\hline $7-8.4 .2$ & 131839 & 2-part & 15 & 1.94 & 0.2 & $\mathrm{~S}$ & Yes & - & Y \\
\hline $7-8.6 .2$ & 103104 & 3-part & 15 & 1.94 & 0.2 & S & Yes & - & Y \\
\hline $7-8.6 .8 .3$ & 142759 & 3-part & 5.5 & 1.94 & 0.2 & $\mathrm{~S}$ & No & - & $\mathrm{N}$ \\
\hline 7-Change \#50 & 131504 & 3-part & 9.5 & 1.94 & 0.2 & $\mathrm{~S}$ & Uncertain & - & Pulsy \\
\hline 7-Change \#51 & 134344 & 3-part & 12 & 1.94 & 0.2 & S & Yes & - & Y \\
\hline $7-8.8 .2$ & 140601 & 4-part & 15 & 1.94 & 0.2 & S & Yes & - & Y \\
\hline $7-8.10 .2$ & 102228 & 6-part & 15 & 1.94 & 0.2 & S & Yes & - & Y \\
\hline 7-Change \#79 & 152733 & 6-part & 9.5 & 1.94 & 0.2 & S & No & - & Pulsy \\
\hline 7-Change \#80 & 154932 & 6-part & 10.5 & 1.94 & 0.2 & $\mathrm{~S}$ & No & - & Pulsy \\
\hline 7-Change \#82 & 085206 & 6-part & 11.5 & 1.94 & 0.2 & S & No & - & Pulsy \\
\hline 7-Change \#83 & 091401 & 6-part & 12 & 1.94 & 0.2 & $\mathrm{~S}$ & Uncertain & - & Pulsy \\
\hline
\end{tabular}




\begin{tabular}{cccccccccc}
\hline Test Line & $\begin{array}{c}\text { Time } \\
\text { Stamp }\end{array}$ & Simulant & $\begin{array}{c}U_{o} \\
(\mathrm{~m} / \mathrm{s})^{(\mathrm{b})}\end{array}$ & $\begin{array}{c}d_{o} \\
(\text { inch) }\end{array}$ & PVF & Firing & $\begin{array}{c}\text { Surface } \\
\text { Flow }\end{array}$ & $\begin{array}{c}\text { Cloud } \\
\text { Height }^{(\mathrm{c})}\end{array}$ & $\begin{array}{c}\text { Turbulence at } \\
\text { Top Probe }^{(\mathrm{d})}\end{array}$ \\
\hline 7-9.2.2-E & 132953 & 6-part & 12 & 1.94 & 0.2 & $\mathrm{~S}$ & Yes & - & $\mathrm{Y}$ \\
7-9.2.3-E & 143502 & 6-part & 13 & 1.94 & 0.2 & $\mathrm{~S}$ & Yes & - & $\mathrm{Y}$ \\
\hline
\end{tabular}

(a) Compiled from Bontha et al. (2015) (Appendix G), Data Acquisition System (DAS) test logs, and run sheets. FIO.

(b) Nozzle velocity is the "Actual PJM Velocity (m/s)" reported in Bontha et al. (2015) or from DAS test logs. Target velocities for run sheets 1 through 6 were $6.3,8.0,9.4,12.0$, and $15.0 \mathrm{~m} / \mathrm{s}$ with $d_{o}=1.94$ and 3.8, 5.7, 7.2, 9.4 , and $10.5 \mathrm{~m} / \mathrm{s}$ for $d_{o}=2.5 \mathrm{~m} / \mathrm{s}$.

(c) From Bontha et al. (2015), Appendix G, based on side cameras 1 and 2 reported in inches above the seam. Cloud height not consistently reported for run sheet 7 and not observed/relevant to run sheet 3 . Surface flow at $15 \mathrm{~m} / \mathrm{s}$ confirmed in email from Fred Damerow on 4/29/2015 at 10:09 am.

(d) Observed from the magnitude and frequency of the instantaneous velocity as compared to the local average velocity at the central upper probe (Valeport 0579 ). The Valeport probe 0581 at the seam along the centerline was found to be bent during run sheet 7 testing (email from John Geeting to Kurt Recknagle on 3/24/2015 at 3:34 pm with attached photo dated 3/23/2015 at 9:25 am). Though the influence of bending remains unclear, the velocity measurements do not of themselves indicate failure of this probe.

(e) Glass powder added consecutively in three steps of 127,127, $205.9 \mathrm{~kg}$ for concentrations of 1.27, 2.54, and $4.59 \mathrm{wt} \%$, respectively, per email from Loni Peurrung on 4/27/2015 at 9:35 am.

Analysis of the data from the $8 \mathrm{ft}$ test vessel is shown in Figure 2.13 in pairs of panels. The panel on the left shows the data for which surface flow was observed visually and the panel on the right shows data for which surface flow was not observed visually as recorded in Table 2.3. The first pair of panels (a and b) displays data from the two-part simulant, the second pair represents data for the KB slurry simulant, and the third pair represents data from the multipart simulants of run sheet 7 . The data for which surface flow was achieved was fit with Eq. (2.1) as described above. The panels on the left show the fits to be reasonable. The modest curvature of these fits arises from the presence of a significant virtual center as summarized in Table 2.4. The same curves are shown in the panels on the left. Here the data deviates significantly from the curves that represent surface flow, particularly at the higher elevations. The reasons for these deviations are the focus of Sections 4 through 7. 

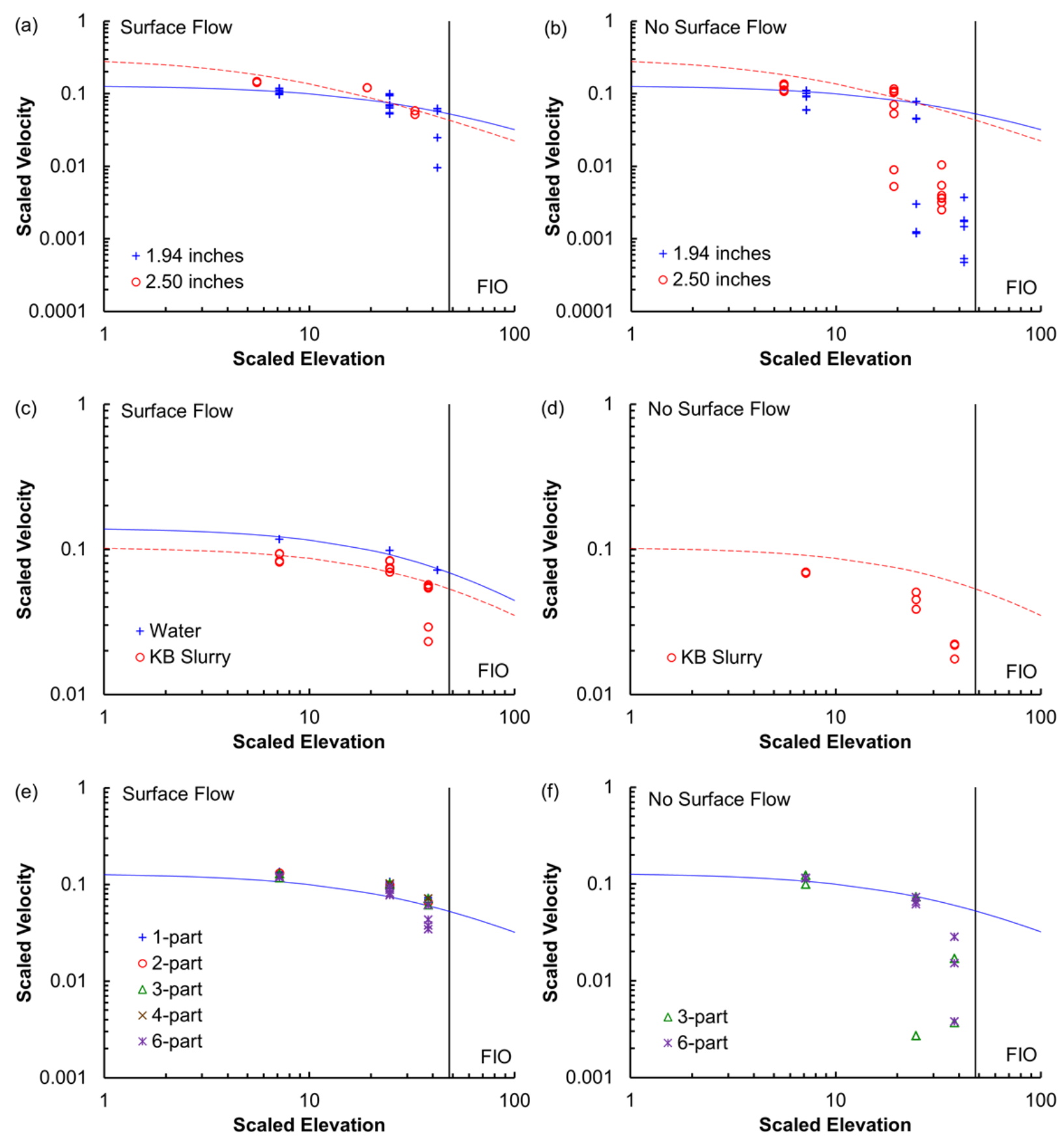

Figure 2.13. Experimental Data from the $8 \mathrm{ft}$ Test Vessel with Central Upwell Velocity and Elevation Scaled on a Nozzle Velocity (see Table 2.3) and the Nozzle Diameter (1.94 inches except where listed), Respectively, for ( $a$ and $b$ ) the Two Part Simulant of Run Sheets 1 and 2 at Two Diameters, (c and d) the KB Slurry of Run Sheet 3 and Water, and (e and f) the Multipart Simulants of Run Sheet 7. Panels a, c, and e include data for which surface flow was observed (fits do not include velocities below $u_{m, c} / U_{o}<0.05$; surface flow not reported for water) and panels $\mathrm{b}, \mathrm{d}$, and f represent data for which surface flow was not observed. Vertical line at $z / d_{o}=48$ represents the dimensionless fill height of the proposed standard high solids vessel and the geometrically scaled $8 \mathrm{ft}$ test vessel. 
Although velocity probe data cannot be collected precisely ${ }^{1}$ at the surface of the vessel and a higher fill height may have added more confidence, data were collected out to $z / d_{o}=42$ in run sheet $1, z / d_{o}=24$ in run sheet 2 , and $z / d_{o}=38$ for run sheets 3 and 7 and clearly approach the nominal fill height. Figure 2.13, therefore, affirms that the data for the $8 \mathrm{ft}$ vessel fall on free jet curves for both Newtonian and non-Newtonian simulants, and that these curves may be reasonably extrapolated to the fluid surface in many cases. This analysis does not support the premise that this jet scaling is a distinctive phenomenon exclusive to Newtonian or non-Newtonian simulants.

Table 2.4 summarizes the essential parameters from fitting Eq. (2.1) using Eq. (2.2) for all of the available data sets. When surface flow is achieved with Newtonian simulants, the value of $h_{c}$ ranges from 2.41 to 5.58 , and $h_{c}$ generally remains less than 1 when surface flow is not achieved. Caution should be exercised in using smaller values of $h_{c}$ because they tend to be associated with curvature that calls into question the linearity asserted in Eq. (2.2) (see Sections 5 and 6 for the development of jet velocity equations that account for curvature). When surface flow is achieved, $z_{o} / d_{o}$ is generally positive. Although this would suggest that $h_{c}$ and $z_{o} / d_{o}$ may serve as a success metric, neither of these values have yet been predicted a priori from first principles or directly from operating conditions for tertiary jets (this is the purpose of Section 3), and the effect of head shape on virtual origins remains unknown. Nevertheless, $z_{o} / d_{o}$ is clearly nonzero and significant and cannot, therefore, be excluded a priori unlike prior analyses of primary or secondary jets ${ }^{2}$ (Rajaratnam 1976, pp. 48 and 235).

\footnotetext{
${ }^{1}$ Because fluid associated with the pulse volume fraction must be suctioned into the PJM, the liquid level in these tests decreases below the nominal fill height. This leaves some velocity probes too close to the nominal fill height dry. The inability to establish a baseline velocity prior to the pulse leaves such data less reliable.

${ }^{2}$ The central upwell is a tertiary jet. A primary jet issues directly from a nozzle. A secondary jet results from the impingement of a primary jet on a surface. The central upwell is a tertiary jet because it arises from the impingement of secondary radial jets.
} 
Table 2.4. Central Upwell Fits ${ }^{(a)}$

\begin{tabular}{|c|c|c|c|c|c|}
\hline Reference & Constituents & $\begin{array}{l}\text { Surface } \\
\text { Flow }\end{array}$ & $\begin{array}{c}\text { Average } \\
h_{c}\end{array}$ & $\begin{array}{c}\text { Average } \\
z_{o} / d_{o}\end{array}$ & $\begin{array}{c}z_{o} \\
\text { (inch) }\end{array}$ \\
\hline Bontha et al. (2003a) & Water & NR & 4.64 & 15.8 & 63.1 \\
\hline Bontha et al. (2003b) & Water & NR & 2.64 & 5.14 & 20.5 \\
\hline Bontha et al. (2003b) & $10 \mu \mathrm{m}$ glass beads $5 \mathrm{wt} \%$ & Y & 2.55 & 6.31 & 25.2 \\
\hline Bontha et al. (2003b) & $10 \mu \mathrm{m}$ glass beads $20 \mathrm{wt} \%$ & $\mathrm{Y}$ & 2.48 & 5.67 & 22.7 \\
\hline Bontha et al. (2003b) & $35 \mu \mathrm{m}$ glass beads $5 \mathrm{wt} \%$ & Y & 2.69 & 6.19 & 24.7 \\
\hline Bontha et al. (2003b) & $35 \mu \mathrm{m}$ glass beads $20 \mathrm{wt} \%$ & Y & 2.46 & 5.79 & 23.2 \\
\hline Bamberger et al. (2005) & KB slurry, APEL, $U_{o}>10 \mathrm{~m} / \mathrm{s}$ & $\mathrm{Y}$ & 1.50 & -5.19 & -4.57 \\
\hline Bamberger et al. (2005) & KB slurry, APEL, $U_{o}<10 \mathrm{~m} / \mathrm{s}$ & Y & 0.209 & -20.4 & -18.0 \\
\hline Bamberger et al. (2005) & KB slurry, LTTS, $U_{o}>7 \mathrm{~m} / \mathrm{s}$ & Y & 2.07 & -5.19 & -20.8 \\
\hline Bamberger et al. (2005) & KB slurry, LTTS, $U_{o}<7 \mathrm{~m} / \mathrm{s}$ & $\mathrm{N}$ & 0.290 & -10.2 & -41.0 \\
\hline Commissioning $^{(\mathrm{b})}$ & Water & NR & 6.49 & 46.1 & 89.3 \\
\hline Run sheet $1^{(b)}$ & Glass powder, $\mathrm{Al}_{2} \mathrm{O}_{3}$ & $\mathrm{Y}$ & 4.26 & 32.8 & 63.5 \\
\hline Run sheet $1^{(\mathrm{b})}$ & Glass powder, $\mathrm{Al}_{2} \mathrm{O}_{3}$ & $\mathrm{~N}$ & 0.0349 & -8.76 & -17.0 \\
\hline Run sheet $2^{(\mathrm{c})}$ & Glass powder, $\mathrm{Al}_{2} \mathrm{O}_{3}$ & Y & 2.41 & 7.65 & 19.1 \\
\hline Run sheet $2^{(c)}$ & Glass powder, $\mathrm{Al}_{2} \mathrm{O}_{3}$ & $\mathrm{~N}$ & 0.109 & -7.63 & -19.1 \\
\hline Run sheet $3^{(b)}$ & KB slurry, $U_{o} \sim 12-15 \mathrm{~m} / \mathrm{s}$ & $\mathrm{Y}$ & 5.29 & 51.1 & 99.0 \\
\hline Run sheet $3^{(\mathrm{b})}$ & KB slurry, $U_{o} \sim 8 \mathrm{~m} / \mathrm{s}$ or A2 firing & $\mathrm{N}$ & 0.915 & 3.04 & 5.89 \\
\hline Run sheet $7^{(\mathrm{b})}$ & Glass powder, $1.27 \mathrm{wt} \%, U_{o}=15.0 \mathrm{~m} / \mathrm{s}$ & Y & 4.76 & 26.9 & 52.1 \\
\hline Run sheet $7^{(\mathrm{b})}$ & Glass powder, $2.54 \mathrm{wt} \%, U_{o}=15.0 \mathrm{~m} / \mathrm{s}$ & $\mathrm{Y}$ & 5.10 & 29.4 & 57.1 \\
\hline Run sheet $7^{(\mathrm{b})}$ & Glass powder, $4.59 \mathrm{wt} \%, U_{o}=15.0 \mathrm{~m} / \mathrm{s}$ & Y & 4.98 & 27.6 & 53.5 \\
\hline Run sheet $7^{(\mathrm{b})}$ & Glass powder, $\mathrm{W},{ }^{(\mathrm{d})} U_{o}=15.0 \mathrm{~m} / \mathrm{s}$ & Y & 4.14 & 22.3 & 43.2 \\
\hline Run sheet $7^{(\mathrm{b})}$ & Glass powder, $\mathrm{W}^{(\mathrm{d})} \mathrm{SiC}, U_{o} \geq 12.0 \mathrm{~m} / \mathrm{s}$ & Y & 4.54 & 27.0 & 52.4 \\
\hline Run sheet $7^{(\mathrm{b})}$ & Glass powder, $\mathrm{W}^{(\mathrm{d})} \mathrm{SiC}, U_{o} \leq 9.5 \mathrm{~m} / \mathrm{s}$ & $\mathrm{N}$ & 0.188 & -0.347 & -0.674 \\
\hline Run sheet $7^{(\mathrm{b})}$ & $\begin{array}{l}\text { Glass powder, } \mathrm{W},{ }^{(\mathrm{d})} \mathrm{SiC} \text {, beads, } U_{o}=15.0 \\
\mathrm{~m} / \mathrm{s}\end{array}$ & $\mathrm{Y}$ & 5.58 & 36.3 & 70.4 \\
\hline Run sheet $7^{(b)}$ & $\begin{array}{l}\text { Glass powder, } \mathrm{W},{ }^{(\mathrm{d})} \mathrm{SiC} \text {, beads, } \mathrm{Al}_{2} \mathrm{O}_{3} \text {, } \\
\text { sand, } U_{o} \geq 12.0 \mathrm{~m} / \mathrm{s}\end{array}$ & $\mathrm{Y}$ & 4.35 & 28.7 & 55.6 \\
\hline Run sheet $7^{(\mathrm{b})}$ & $\begin{array}{l}\text { Glass powder, } \mathrm{W} \text {, }{ }^{(\mathrm{d})} \mathrm{SiC} \text {, beads, } \mathrm{Al}_{2} \mathrm{O}_{3} \text {, } \\
\text { sand, } U_{o} \leq 11.5 \mathrm{~m} / \mathrm{s}\end{array}$ & $\mathrm{N}$ & 0.287 & -9.41 & -18.3 \\
\hline
\end{tabular}

APEL is Applied Process Engineering Laboratory.

(a) Values $u_{m, c} / U_{o}<0.05$ were not included in the fits where surface flow was achieved (they were included when surface flow was not achieved) as they present significant curvature on plots of $U_{o} / u_{m, c}$ versus $z / d_{o}$, which indicates that another flow regime has set in. This occurred at the upper probes for tests 1-8.3.3, 1-8.7.2, 3-8.58.3, 3-8.58.4, 7-change \#83, 7-9.2.2-E, and 7-9.2.3-E. A change in regimes is perhaps expected for the KB slurry of run sheet 3 as indicated in Section 6. The remainder indicate that the jet flow itself did not achieve surface flow, but that jet-induced motion, perhaps akin to the coherent motion near the cloud height, did reach the surface.

(b) Eight foot vessel test data from run sheets 1, 2, 3 and 7 FIO.

(c) Run sheet 2 differs from the other run sheets as abstracted here in that it has a nozzle diameter of $d_{0}=2.5$ inches instead of 1.94 inches. Eight foot vessel test data from run sheets 1, 2, 3 and 7 FIO.

(d) $\mathrm{W}$ denotes the dense tungsten component, tungsten carbide with a density of $9.6 \mathrm{~g} / \mathrm{cm}^{3}$.

\subsection{Minimum Turbulent Velocities and the Poirier-Etchells Criterion}

An essential question that arises from the analysis of experimental velocity data is what local velocity is necessary to sustain jet turbulence. How much can the jet velocity attenuate before turbulence vanishes? This is particularly important on a log-log plot where the velocity never completely vanishes. 
This question is addressed by the Poirier-Etchells criterion. ${ }^{1}$ This criterion asserts that at any location the local velocity must be in excess of a cutoff velocity to remain turbulent. When coupled with the free jet velocity equation that relates the local velocity to elevation (Eq. (2.1)), the elevation at which turbulence will fail and consequently the turbulent jet will fail may be determined. This elevation may be compared to the elevation of the fluid surface to predict whether the upwell will reach the surface as a metric of surface flow. Each part of this argument is now evaluated in turn.

First, the local velocity metric asserts that turbulence persists where the local jet velocity remains in excess of a specific velocity, originally given as $0.5 \mathrm{ft} / \mathrm{s}(0.15 \mathrm{~m} / \mathrm{s})$. This is a purely empirical, experienced based rule of thumb. The cutoff velocity applies not only at the upper surface but may apply at any point throughout the vessel. For example, Figure 2.14a compares the local velocity at both the top and middle probes to readings of turbulence at each probe in the $8 \mathrm{ft}$ vessel tests. The turbulence metric is unity if the temporal velocity trace shows signs of turbulence (e.g., instant velocities that differ significantly from the average velocity), zero if the temporal trace of the local velocity shows no sign of turbulence, and 0.5 if the jet is pulsy for the Newtonian simulants or the turbulence is attenuating for the non-Newtonian simulant. When the local velocity remains greater than $0.18 \mathrm{~m} / \mathrm{s}$ the jet shows indications of turbulence, which is in approximate agreement with the $0.50 \mathrm{ft} / \mathrm{s}(0.15 \mathrm{~m} / \mathrm{s})$ cutoff originally proposed, though the jet flow may remain pulsy or attenuated. Conversely, when the local velocity is greater than $0.33 \mathrm{~m} / \mathrm{s}$ for the Newtonian simulant or $0.42 \mathrm{~m} / \mathrm{s}$ for the non-Newtonian simulant, the upwell jet is fully turbulent. The difference in the cutoff velocities suggests that the cutoff velocity may depend on the material through which the flow is penetrating, but there is no a priori reason to expect that the cutoff velocity for the central upwell would depend on vessel dimensions because turbulence is a property of the flow unless the vortex itself is large enough to be confined by the vessel or vessel internals. This intimates the possibility of evaluating the cutoff velocity from small scale measurements. Additional modeling of the turbulence structure and stability in the presence of a full range of slurries may predict the cutoff velocity directly as a function of slurry properties (Landa et al. 2005). For wall jets, different cutoff velocities may apply because the turbulence structure may be significantly affected by the presence of a bounding surface (Bird et al. 2002; Masuyama et al. 1980).

The cutoff velocity dictates the conditions under which the jet begins to fail, but does not predict the location within mixing vessels where the failure may occur. This is important because if the jet velocity drops below the cutoff velocity at an elevation significantly below the fluid surface, then the upwell may not disturb or induce flow at the surface. Fortuitously, Eq. (2.1) provides a relationship between a decaying centerline velocity and elevation for a free jet. One may then construct a ratio of the centerline velocity to the cutoff velocity, $u_{c o}$. When this ratio meets or exceeds one, the jet should reach the surface. This ratio may be rearranged as

$$
\frac{u_{m, c}}{u_{c o}}=h_{c} \frac{U_{o}}{u_{c o}}\left(\frac{z+z_{o}}{d_{o}}\right)^{-1} \geq 1
$$

Remarkably, this dimensionless group (i.e., the one in the middle) is independent of geometric scale (as one might expect of a useful rule of thumb) where $z_{o} / d_{o}$ does not change and $U_{o}$ is held constant, because $h_{c}$ will be shown previously to be independent of vessel geometric scaling for a free jet (see Section 3 ). Sections 5-6 present alternative velocity profiles from which $h_{c}$ can be approximated in the presence of

\footnotetext{
${ }^{1}$ Personal conversation with Michael Poirier, 3/4/2015.
} 
strong negative buoyancy or yield stresses. This may be necessary where $h_{c}$ is small to account for the curvature found in fitting Eq. (2.2) to slurries.

To evaluate whether this dimensionless criterion may be used to predict observations of upwelling, surface flow or breakthrough of the central upwell at the fluid surface in WTP vessels, Figure 2.14b shows this dimensionless group versus a surface flow metric. Essentially, the metric is one if turbulent jet flow may be asserted with confidence, zero for no flow at the fluid surface and 0.5 for closer calls. Figure 2.14b shows that this group does a remarkably good job in estimating whether surface flow occurs using the two velocity cutoffs for the two types of materials.
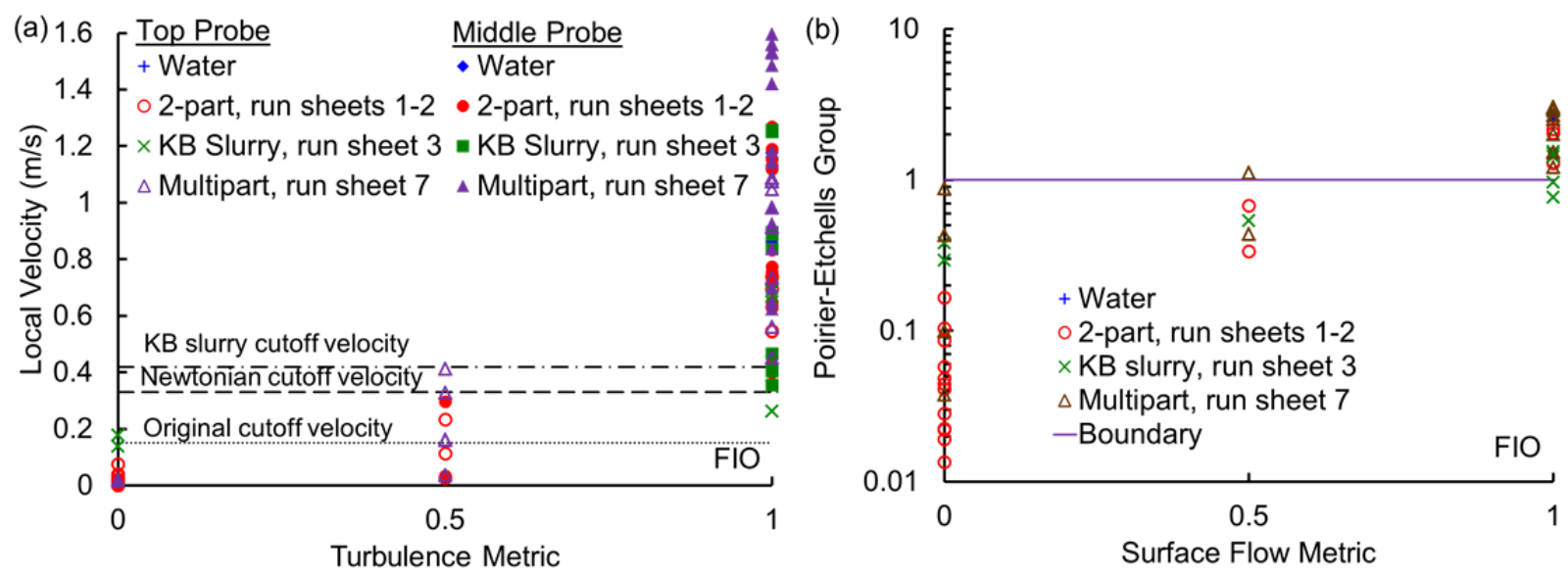

Figure 2.14. (a) The Local Velocity versus Turbulence Metric Described in the Text for Various Simulants at Two Probe Elevations (top 60-68 inches above seam and middle 34 inches above seam). The original Poirier-Etchells cutoff velocity of $0.50 \mathrm{ft} / \mathrm{s}(0.15 \mathrm{~m} / \mathrm{s})$ (dots) and the revised velocity cutoffs of $0.33 \mathrm{~m} / \mathrm{s}$ (dashed) and $0.42 \mathrm{~m} / \mathrm{s}$ (dash dot) for Newtonian and KB slurries, respectively, are shown as horizontal lines. (b) The Dimensionless Poirier-Etchells Group Differentiated on the Surface Flow Metric Described in the Text.

A test of this criterion may be predicting the cloud height. Setting the dimensionless group in Eq. (2.12) to 1 and solving for the elevation where this criterion fails may be a predictor of cloud height, $z_{\text {cloud. }}$ In scaled form,

$$
\frac{z_{\text {cloud }}}{d_{o}}=h_{c} \frac{U_{o}}{u_{c o}}-\frac{z_{o}}{d_{o}}
$$

Predicted cloud heights were compared to the cloud heights measured in run sheets 1 and 2 and a least squares technique was used to estimate the cutoff velocity as $u_{c o}=0.60$ and $0.48 \mathrm{~m} / \mathrm{s}$ for the 1.94 and 2.50 inch nozzles, respectively. Figure 2.15 a compares these two with the single value of $u_{c o}$ estimated for each of the two nozzle diameters. The figure shows that the agreement is reasonable with scatter anticipated for a rule of thumb. Figure $2.15 \mathrm{~b}$ shows that this simple projection reasonably but not perfectly differentiates surface flow from no surface flow. The lower cutoff velocity of $u_{c o}=0.33 \mathrm{~m} / \mathrm{s} \mathrm{did}$ not accurately predict either the cloud height or surface flow conditions. This indicates that other effects also contribute to the cloud height as discussed in Section 5.2. 

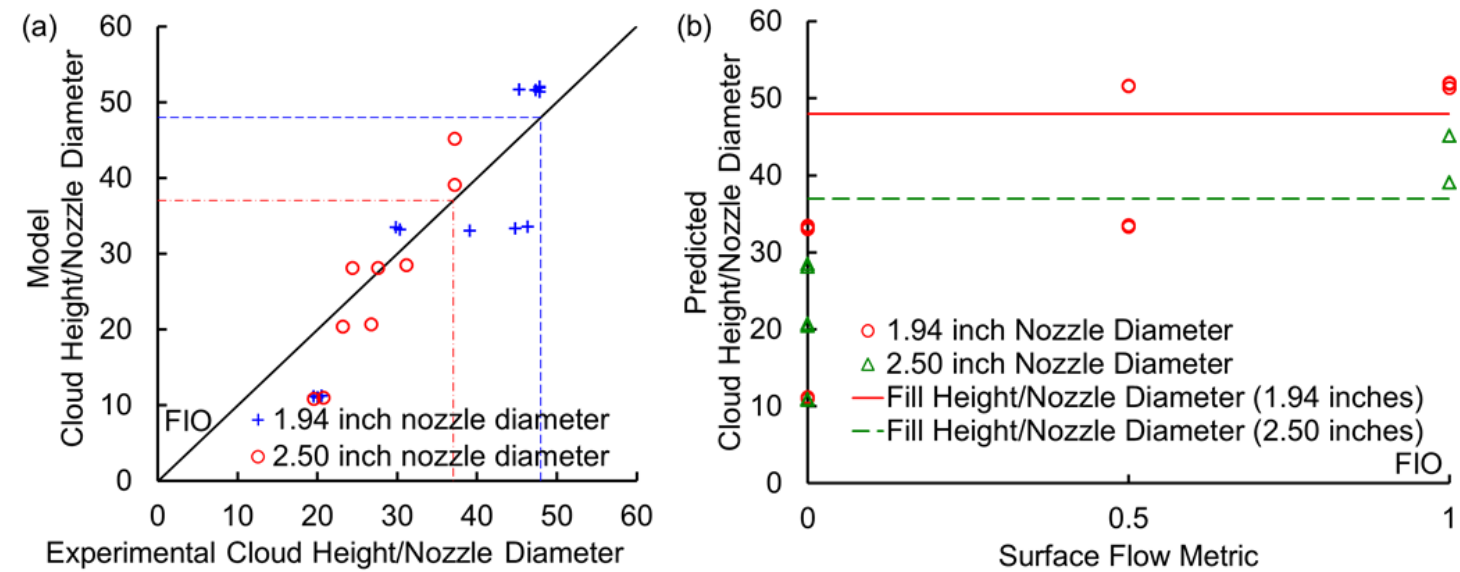

Figure 2.15. (a) Predicted versus Experimental Cloud Height Scaled on Nozzle Diameter with $u_{c o}=0.60$ and $0.48 \mathrm{~m} / \mathrm{s}$ for the 1.94 and 2.50 inch Nozzles, Respectively, for Tests from Run Sheets 1 and 2 Listed in Table 2.3 Compared to Fill Height/Nozzle Diameter for $d_{o}=1.94$ inches (dash, blue) and 2.50 inches (dash dot, red). (b) Predicted Cloud Height Differentiated on the Surface Flow Metric of Table 2.3 with $u_{c o}$ as in Panel a.

\subsection{Summary}

The central upwell may be approximated as a free jet in both Newtonian and non-Newtonian slurries at sufficiently high velocities. Equation 2.1 predicts jet flow when the jet coefficient and depth of the virtual origin are known. See Section 3 for formulas for jet coefficients of free jets, but use of Eq. 2.1 is inappropriate when the jet velocity is insufficient. See Sections 5 and 6 for jet flows at lower velocities where negative buoyancy and yield stresses are influential, making the jet not "free". What constitutes sufficient jet velocity is not determined herein quantitatively.

The Poirier-Etchells criterion asserts a cutoff velocity above which jet turbulence persists. The jet is predicted to reach the surface when the dimensionless group in Eq. 2.12 exceeds one. There is insufficient evidence to reliably predict performance of full scale jets from smaller scale data because the cutoff velocity has not been shown to be scale independent experimentally. This limitation may be overcome by analyzing experimental data at other scales to determine the cutoff velocity, quantifying the radial extent of vortices experimentally, and by modeling vortex size. 



\subsection{Upwell Velocity Profile from Momentum Conservation}

Conservation of momentum arguments may be used to describe and predict the jet flow profile of the central upwell. This section evaluates and determines how the free jet profile scales between vessels. The momentum flux at any point is $\rho u^{2}$, where $\rho$ is the local density and $u$ is the local velocity. This product is used to predict the velocity profiles based on conservation of momentum for various conditions.

\subsection{The Upwell Velocity Profile}

The momentum flux leaving a nozzle with velocity $U_{o}$ and slurry density $\rho_{o}$ is then $\rho_{o} U_{o}^{2}$. To obtain the rate of momentum crossing a control surface, the momentum flux is multiplied by its area. For a nozzle diameter of $d_{o}$, the relevant area is $\pi d_{o}^{2} / 4$. One might imagine that with $N$ nozzles, the total rate of momentum contributing to the central upwell is $\pi N \rho_{o} U_{o}^{2} d_{o}^{2} / 4$. However, at least some of the momentum goes toward the wall instead of the center. ${ }^{1}$

To determine how much goes toward the center, consider a control volume of a regular n-gon in the plane perpendicular to the PJM tubes with vertices at the PJM centerlines. This n-gon is a square with four PJMs and a hexagon with six PJMs and has a curvilinear bottom that hugs the fluid side of the vessel surface. Each nozzle contributes $(N-2) / 2 N$ of its flow to the central upwell and $(N+2) / 2 N$ to the flow along the wall, which is precisely true for a vessel with a flat bottom with nozzles sufficiently far away from a wall. However, some of the outwardly directed PJM flow may go up along the side wall and then return toward the center, providing a crossflow. ${ }^{2}$ If the fraction of the flow that returns to contribute its momentum to the central upwell is $f_{r}$, then $f_{N}=(N-2) / 2 N+f_{r}(N+2) / 2 N$ is defined as the total fraction of the PJM flow that contributes to the central upwell. (The value of $f_{N}$ is estimated from historical and $8 \mathrm{ft}$ test below.) For $N$ nozzles, the rate of momentum addition to the control volume is then

$$
M_{o}=\frac{\pi}{4} N f_{N} \rho_{o} U_{o}^{2} d_{o}^{2}
$$

In the absence of constraints, a larger nozzle diameter for the same velocity would give the most momentum and, therefore, be preferred. However, if the volumetric flow rate for each PJM, $Q$, is fixed, there is a tradeoff between the velocity and diameter. Replacing the velocity with $U_{o}=4 Q / \pi d_{o}^{2}$ gives

$$
M_{o}=\frac{4 N f_{N} \rho_{o} Q^{2}}{\pi d_{o}^{2}}
$$

\footnotetext{
${ }^{1}$ If the PJMs were buttressed against the vessel side wall such that all of the flow were toward the center, then the rate of momentum addition to the control volume becomes $\pi \rho U_{o}^{2} d_{o}^{2} N / 4$, which is likely the source of the arguments given in Bamberger et al. (2005) and Gauglitz et al. (2009). However, the PJMs are separated from the vessel wall and the proposed standard high solids vessel floor curves with an elliptical head.

${ }^{2}$ Chapter 9 of Rajaratnam (1976) discusses crossflow. Crossflow leads to deflection of the jet profile in the direction of the crossflow. This would suggest a marginally larger angle of incidence between the centerline (now curving) of the jet and the vessel bottom, which would increase the value of $h_{r}$ in Eq. (3.13) toward the center of the vessel and decrease it toward the vessel wall (Beltaos 1975; Beltaos and Rajaratnam 1977).
} 
which suggests that decreasing the diameter will increase the rate of momentum addition under this constraint. ${ }^{1}$ Similarly, recognizing that the volume of fluid that exits the PJMs is approximately equal to the flow rate times the drive time, $t_{D}$, times the number of PJMs or the pulse volume fraction (PVF) ${ }^{2}$ or drive volume fraction (DVF) multiplied by the total vessel volume $\left(V_{\text {vessel }}\right)$. The rate of momentum addition is then

$$
M_{o}=\frac{4 f_{N} \rho_{o} P V F^{2} V_{\text {vessel }}^{2}}{N \pi d_{o}{ }^{2}{t_{D}}^{2}}
$$

When scaling between 8 and $16 \mathrm{ft}$ vessels, the volume will approximately cube, the nozzle diameter will approximately double, and the drive time will approximately double while holding all else constant, so that the rate of momentum addition will be substantially larger than in the $8 \mathrm{ft}$ vessel. More momentum addition means more upwell velocity and more particle transport. Of course, in the larger vessel the momentum has larger forces to overcome. ${ }^{3}$

The objective here then is to use conservation of momentum to find an expression for $u_{m, c}$ from the momenta of the $N$ jets, assuming the jet to be free. Jet flows that are not free are covered in Sections 4 through 7. The momentum leaving the control volume through the central upwell is

$$
2 \pi u_{m, c}^{2} \rho \int_{0}^{\infty} f_{c}^{2}\left(\eta_{c}\right) r d r
$$

Setting the two rates of momenta (i.e., jet forces) equal gives

$$
2 \pi \delta_{c}{ }^{2} u_{m, c}{ }^{2} \rho \int_{0}^{\infty} f_{c}{ }^{2}\left(\eta_{c}\right) \eta_{c} d \eta_{c}=\frac{\pi}{4} N f_{N} \rho_{o} U_{o}{ }^{2} d_{o}{ }^{2}
$$

where $\eta_{c}=r / \delta_{c}$ in the absence of surface or body force (see Meacham et al. 2012, Eq. 7-6). The integral becomes a constant

$$
\psi_{2,1}=\int_{0}^{\infty} f_{c}^{2}\left(\eta_{c}\right) \eta_{c} d \eta_{c}
$$

The values of $\psi_{2,1}$ may be estimated directly from the radial velocity profiles given in Section 2.2.

Table 3.1 gives $\psi_{2,1}$ constant for the four theoretical models of Eqs. (2.5) through (2.8). Yet, the historical data analyzed in Figure 2.4 suggests that the radial profiles may be steeper for slurries than the theoretical values of fluid-only jets would suggest. Values of $\psi_{2,1}$ were estimated from the normalized Fermi-Dirac function fits of Figure 2.4 as also shown in Table 3.1. Because the radial distribution of velocity profiles

\footnotetext{
${ }^{1}$ This is consistent with the findings in CCN 272775.

${ }^{2}$ This definition of PVF does not include the vent time as some definitions do and is, therefore, equal to the DVF. Please see CCNs 272774 and 272778 for further discussion of pulse volume fraction. PVF will be retained for notational convenience.

${ }^{3}$ These are mostly surface forces so they would at most scale with the square of the vessel dimension canceling out the effect, but only if these surfaces or shear forces are significant; they are not for a free jet.
} 
was not measured in the $8 \mathrm{ft}$ test vessel, the values of this constant cannot be estimated for the particle compositions considered in these vessels.

Table 3.1. Constants Derived from Radial Distributions

\begin{tabular}{llccc}
\hline Reference & Model or Constituent & $\psi_{2,1}$ & $\eta_{c}^{99(\mathrm{a})}$ & $\psi_{1,1}{ }^{(\mathrm{b})}$ \\
\hline Wiegel (1964) & Gaussian (Eq. (2.5)) & $1 / \operatorname{Ln}(16) \sim 0.361$ & 2.578 & $1 / \operatorname{Ln}(4) \sim 0.721$ \\
Rajaratnam (1976) & Squire and Trouncer (Eq. (2.6)) & $3 / 4-4 / \pi^{2} \sim 0.345$ & 1.872 & $1-4 / \pi^{2} \sim 0.595$ \\
Rajaratnam (1976) & Prandt1/Tollmien (Eq. (2.7)) ${ }^{(\mathrm{a})}$ & 0.349 & 2.493 & 0.738 \\
Bird et al. (2002) & Boussinesq/Goertler (Eq. (2.8)) & $\left(1+2^{1 / 2}\right) / 6 \sim 0.402$ & 4.661 & $\left(1 / 2+1 / 2^{1 / 2}\right)$ \\
& & & & $\sim 1.207$ \\
Bontha et al. (2003b) & Water & 0.383 & 2.716 & 0.762 \\
Bontha et al. (2003b) & $10 \mu \mathrm{m}$ glass beads 5 wt\% & 0.378 & 2.634 & 0.736 \\
Bontha et al. (2003b) & $10 \mu \mathrm{m}$ glass beads 20 wt\% & 0.363 & 2.194 & 0.621 \\
Bontha et al. (2003b) & $35 \mu \mathrm{m}$ glass beads 5 wt\% & 0.369 & 1.948 & 0.574 \\
Bontha et al. (2003b) & $35 \mu \mathrm{m}$ glass beads 20 wt\% & 0.389 & 2.795 & 0.787 \\
Bamberger et al. (2005) & KB slurry & 0.398 & 1.597 & 0.528 \\
\hline
\end{tabular}

(a) The polynomial fit of Eq. (2.7) has a minimum of 0.00506 at $\eta_{c}=2.663$. Integration proceeds to this minimum.

(b) Defined in Section 7.

Solving for $u_{m, c}$ then gives

$$
u_{m, c}=\frac{c_{j}}{\beta_{c}} \sqrt{\frac{N f_{N}}{8 \psi_{2,1}} \frac{\rho_{o}}{\rho}} \frac{U_{o} d_{o}}{z+z_{o}}
$$

where $c_{j}$ represents an additional jet fitting constant, where needed to account for variations from a circular shape (Rajaratnam 1976), the inclusion of momentum carrying particles if not included explicitly (see Section 5), influences of yield stresses, etc. (This coefficient is evaluated explicitly in Table 3.3.) Scaling the local velocity on the nozzle velocity and the elevation on the nozzle diameter gives

$$
\frac{u_{m, c}}{U_{o}}=\frac{c_{j}}{\beta_{c}} \sqrt{\frac{N f_{N}}{8 \psi_{2,1}} \frac{\rho_{o}}{\rho}} \frac{1}{z / d_{o}+z_{o} / d_{o}}
$$

from which the jet coefficient may be defined as

$$
h_{c} \equiv \frac{c_{j}}{\beta_{c}} \sqrt{\frac{N f_{N}}{8 \psi_{2,1}} \frac{\rho_{o}}{\rho}}
$$

Please note that viscosity is not a significant factor in these expressions because momentum in the axial direction is conserved regardless of laminar or turbulent viscosity model in the absence of a yield stress, such that the viscous terms vanish in conservation of momentum (Bird et al. 2002). However, turbulent viscosity may play a role in the radial spread through conservation of energy as described in Section 4.3.

The key addition here is a clear expression for how the number of PJMs contributes to the velocity profile. As the number of jets increases, the central velocity should increase, but $f_{N}$ has not previously been estimated. By simple geometric arguments, one may anticipate that for six PJMs, $f_{6}=1 / 3$ because 
only $1 / 3$ of the radial outward flow from a PJM has a vector component of velocity toward the center of the vessel. Similarly in a four-PJM vessel, $f_{4}$ may equal $1 / 4$ because only $1 / 4$ of the radial outward flow has velocity component toward the center of the vessel, giving $f_{6} / f_{4}=4 / 3$. This ratio may be estimated from the water data from historical testing $\left(h_{c}=4.64\right.$, Bontha et al. 2003a) and from the $8 \mathrm{ft}$ test vessel $\left(h_{c}=6.49\right)$ in Table 2.4. Asserting $c_{j}, \beta_{c}, \psi_{2,1}, \rho_{o}$, and $\rho$ to not vary between tests and scales,

$$
\frac{f_{6}}{f_{4}}=\frac{4}{6}\left(\frac{h_{c}(N=6)}{h_{c}(N=4)}\right)^{2}=\frac{4}{6}\left(\frac{6.49}{4.64}\right)^{2}=1.30
$$

Similarly, in the A1 and A2 firing sequences, only three of the PJMs operate at any given time, suggesting an approximate comparison between $N=3$ for A1 or A2 firing and $N=6$ for S firing. ${ }^{1}$ By geometric arguments, $f_{3}=1 / 6$ because only one-sixth of the flow is directed inward toward the vessel center, such that one may anticipate $f_{6} / f_{3}=2$. Comparing test 1-8.3.3 with A1 firing $\left(h_{c}=2.14\right.$ without the top probe that was not fully turbulent, $N=3$ effective) or test 1-8.5.1 with A2 firing $\left(h_{c}=1.96\right)$ to 1-8.4.3 with the $\mathrm{S}$ firing sequence $\left(h_{c}=3.96\right)$ gives $f_{6} / f_{3}=3 / 6(3.96 / 2.14)^{2}=1.71$ or $f_{6} / f_{3}=3 / 6(3.96 / 1.96)^{2}=2.05$, respectively.

The approximate agreement with these simple geometric arguments is remarkable and somewhat unexpected because other factors may influence the momentum available to the central upwell, including curvature of the vessel bottom, peripheral flows, and bottom shear stresses. Nevertheless, this analysis suggests that these factors may cancel out. Mathematically, increasing $f_{N}$ to one by driving all of the flow vectors toward the center would substantially increase the momentum addition to the central upwell. Evaluation of the substantial increases in cloud and "cavern" heights and changes in bottom clearing/motion remains outside the scope of this report.

\subsection{Upwell Velocity Profile Including Shear Along the Vessel Bottom}

This subsection shows that shear stresses along the vessel bottom are small enough to be negligible with respect to the upwell flow. The reader may advance to the next subsection without loss of continuity. The derivation thus far has not included shear stresses along the bottom and the influence of the radial position of the PJM or the standoff distance. To estimate the influence of PJM position on the central upwell, consider momentum lost along the vessel floor due to shear stresses. For convenience to a first order approximation, imagine all the flow approaches the vessel centerline radial as if it were generated by an infinite number of differentially small PJMs in a circle of radius $f_{R} R$. $^{2}$ Momentum is primarily lost between the PJM radius at $f_{R} R$ (where $f_{R}$ lies typically in the range of 0.6 to 0.7 ) and the edge of the central vortex at $f_{u} R$ (see Table 2.1), due to the shear stresses along the vessel bottom, $\tau_{w}$. Integrating over the bottom of the vessel surface converts the stress along the bottom into a frictional force. The domain of integration may be divided into $2 N$ domains that integrate from 0 to $\pi / N$. For

\footnotetext{
${ }^{1}$ The A1 and A2 firing sequences typically have some suction in one set of PJMs while the other set is firing, reducing the momentum available to the central upwell.

${ }^{2}$ An alternative is to set the equations up in the oblate spheroidal coordinate system, do the integrations there, and then convert back into a radial or Cartesian coordinate system as desired. Computational fluid dynamics can of course address this directly and provide corrections. Integration over azimuthal direction would likely not significantly affect the functional form, though variations in constants may arise if completed more rigorously. This derivation suffices to build intuition.
} 
example, with six PJMs, there are 12 identical wedges that span a $30^{\circ}$ angle. Then the rate of momentum loss becomes

$$
M_{\text {lost }}=2 N \int_{0}^{\pi / N} \int_{f_{u} R}^{f_{R} R} \tau_{w} r d r d \theta
$$

The stress along the bottom may be expressed in terms of a skin coefficient of friction, $c_{f}$, as

$$
\tau_{w}=c_{f} \rho_{b} u_{m, r}{ }^{2} / 2
$$

where this density, $\rho_{b}$, is the slurry density along the vessel bottom (which may or may not equal $\rho_{o}$ ), and $u_{m, r}$ is the maximum velocity in the radial velocity profile along (nearly parallel to) the vessel bottom. In the form of Eq. (2.1),

$$
u_{m, r}=\frac{h_{r} U_{o} d_{o}}{r}
$$

where $h_{r}$ is the jet coefficient in the radial direction, $r$ (Beltaos 1975; Rajaratnam 1976; Beltaos and Rajaratnam 1977; Phares et al. 2000), and Rajaratnam (1976) suggests the virtual origin for the radial wall jet remains small enough to be negligible. Substitution and integration yields

$$
M_{\text {lost }}=\pi c_{f} \rho_{b} h_{r}{ }^{2} U_{o}{ }^{2} d_{o}{ }^{2} \ln \left[f_{R} / f_{u}\right]
$$

The momentum available to the rising jet is reduced by $M_{\text {lost }}$ so that conservation of momentum requires

$$
2 \pi \delta_{c}^{2} u_{m, c}{ }^{2} \rho \int_{0}^{\infty} f_{c}^{2}\left(\eta_{c}\right) \eta_{c} d \eta_{c}=\frac{\pi}{4} U_{o}{ }^{2} d_{o}{ }^{2} \rho_{o} N f_{N}\left(1-4 c_{f} \frac{\rho_{b}}{\rho_{o}} \frac{h_{r}{ }^{2}}{N f_{N}} \ln \left(\frac{f_{R}}{f_{u}}\right)\right)
$$

where the additional factor on the right-hand side represents the loss of momentum due to surface shear forces as the radial jet moves from PJM to vessel center. Solving for $u_{m, c}$ gives

$$
u_{m, c}=\frac{c_{j}}{\beta_{c}} \sqrt{\frac{N f_{N}}{8 \psi_{2,1}} \frac{\rho_{o}}{\rho}} \sqrt{1-4 c_{f} \frac{\rho_{b}}{\rho_{o}} \frac{h_{r}^{2}}{N f_{N}} \ln \left(\frac{f_{R}}{f_{u}}\right)} \frac{U_{o} d_{o}}{z+z_{o}}
$$

where $c_{j}$ represents an additional jet fitting constant as needed to fit data. In scaled terms,

$$
\frac{u_{m, c}}{U_{o}}=\frac{c_{j}}{\beta_{c}} \sqrt{\frac{N f_{N}}{8 \psi_{2,1}} \frac{\rho_{o}}{\rho}} \sqrt{1-4 c_{f} \frac{\rho_{b}}{\rho_{o}} \frac{h_{r}^{2}}{N f_{N}} \ln \left(\frac{f_{R}}{f_{u}}\right)} \frac{1}{z / d_{o}+z_{o} / d_{o}}
$$

from which the jet coefficient may be defined as

$$
h_{c} \equiv \frac{c_{j}}{\beta_{c}} \sqrt{\frac{N f_{N}}{8 \psi_{2,1}} \frac{\rho_{o}}{\rho}} \sqrt{1-4 c_{f} \frac{\rho_{b}}{\rho_{o}} \frac{h_{r}^{2}}{N f_{N}} \ln \left(\frac{f_{R}}{f_{u}}\right)}
$$


This shows that including the shear stress modifies the coefficient and does not provide an intercept associated with the distance the jet traveled, as may have been anticipated from prior "cavern" height derivations. ${ }^{1}$ This dimensionless group is $c_{f} \rho_{b} h^{2} \ln \left(f_{R} / f_{u}\right) /\left(\rho_{o} N f_{N}\right)$. Beltaos (1975) gives

$$
c_{f}=0.098 \mathrm{Re}_{\mathrm{o}}^{-1 / 5}
$$

where $\mathrm{Re}_{\mathrm{o}}$ is the nozzle Reynolds number based on the density, velocity, diameter, and viscosity at the nozzle. Beltaos (1975) also gives

$$
h_{r}(\phi)=1.10[1+\cos (\phi)] / \sin ^{1 / 2}(\phi)
$$

in the direction of the vessel center and

$$
h_{r}(\phi)=1.10[1-\cos (\phi)] / \sin ^{1 / 2}(\phi)
$$

in the direction of the vessel wall where $\phi$ is the angle between the centerline of the jet and the vessel bottom. $^{2}$ Because the nozzle Reynolds number is much greater than $1\left(\sim 10^{3}\right.$ to $\left.10^{4}\right)$ and $\rho_{\mathrm{b}} / \rho_{o}, h_{r}$ and $\ln \left(f_{R} / f_{u}\right) / N f_{N}$ are all of order 1 , the addition is marginal in terms of the central upwell. ${ }^{3}$ When $f_{R}=0.6$ as opposed to 0.7, there is less distance and less friction between PJMs and centerline, which suggests a larger scaled upwell velocity. However, curvature along the vessel bottom will enhance $h_{r}$ more with $f_{R}=0.7$ than 0.6. In either case, these changes likely do not make a large difference. Beltaos (1975) and Beltaos and Rajaratnam (1977) argue that the standoff distance for a radial jet is not significant outside the region of impingement, which completes this argument.

The alternative to this approach to evaluate the influence of PJM position and standoff distance using Poreh et al. (1967), which was derived specifically for solids free flows. Kuhn et al. (2013, p. 2.27) start with Eq. 28 in Poreh et al. (1967), which, when rearranged to isolate the shear stress along the bottom, is

$$
\tau_{w}=0.3 \frac{\rho_{b} K}{r^{2}}\left(\frac{\sqrt{K}}{v}\right)^{-0.3}\left(\frac{r}{b}\right)^{-0.3}
$$

where $b$ is the standoff distance. Substituting the kinematic momentum flow $K=C_{k} \pi d_{o}^{2} U_{o}^{2} / 4$ where $C_{k}$ is a nozzle coefficient gives

$$
\tau_{w}=0.3\left(\frac{\pi}{4} C_{k}\right)^{17 / 20} \operatorname{Re}_{o}^{-0.3} \rho_{b} \frac{d_{o}^{2} U_{o}^{2}}{r^{2}}\left(\frac{r}{b}\right)^{-0.3}
$$

Integrating the shear stress over the vessel bottom as before gives the shear friction force as

\footnotetext{
${ }^{1}$ Even if one takes a Taylor series expansion of the second square root, an intercept does not arise.

${ }^{2}$ Streeter (1971) gives a different formula with the same trend. Please note that the $8 \mathrm{ft}$ vessel has a flanged spherical head while the $16 \mathrm{ft}$ standard high solids vessel has a 2:1 elliptical head, which modestly affects the value of $\phi$.

${ }^{3}$ Shear stresses may or may not be significant elsewhere in the vessel, but must play a significant role in particle transport along the vessel bottom. The erosion rate can be determined from first principles by a similar analysis outside the present scope of this report.
} 


$$
M_{\text {lost }}=2 N \int_{0}^{\pi / N} \int_{f_{u} R}^{f_{R} R} \tau_{w} r d r d \theta=2 \pi\left(\frac{\pi}{4} C_{k}\right)^{17 / 20} \rho_{b} d_{o}^{2} U_{o}^{2}\left(\frac{b}{\operatorname{Re}_{o} f_{R} R}\right)^{0.3}\left[\left(\frac{f_{R}}{f_{u}}\right)^{0.3}-1\right]
$$

Conservation of momentum in the same pattern as above returns

$$
\begin{aligned}
& 2 \pi \delta_{c}^{2} u_{m, c}^{2} \rho \int_{0}^{\infty} f_{c}^{2}\left(\eta_{c}\right) \eta_{c} d \eta_{c}= \\
& \quad \frac{\pi}{4} \rho_{o} N f_{N} U_{o}^{2} d_{o}^{2}\left\{1-8\left(\frac{\pi}{4} C_{k}\right)^{17 / 20} \frac{1}{N f_{N}} \frac{\rho_{b}}{\rho_{o}}\left(\frac{b}{\operatorname{Re}_{o} f_{R} R}\right)^{0.3}\left[\left(\frac{f_{R}}{f_{u}}\right)^{0.3}-1\right]\right\}
\end{aligned}
$$

Solving for $u_{m, c}$ gives

$$
u_{m, c}=\frac{c_{j}}{\beta_{c}} \sqrt{\frac{N f_{N}}{8 \psi_{2,1}} \frac{\rho_{o}}{\rho}} \sqrt{1-8\left(\frac{\pi}{4} C_{k}\right)^{17 / 20} \frac{1}{N f_{N}} \frac{\rho_{b}}{\rho_{o}}\left(\frac{b}{\operatorname{Re}_{o} f_{R} R}\right)^{0.3}\left[\left(\frac{f_{R}}{f_{u}}\right)^{0.3}-1\right]} \frac{U_{o} d_{o}}{z+z_{o}}
$$

where $c_{j}$ represents an additional jet fitting constant as needed. In scaled terms,

$$
\frac{u_{m, c}}{U_{o}}=\frac{c_{j}}{\beta_{c}} \sqrt{\frac{N f_{N}}{8 \psi_{2,1}} \frac{\rho_{o}}{\rho}} \sqrt{1-8\left(\frac{\pi}{4} C_{k}\right)^{17 / 20} \frac{1}{N f_{N}} \frac{\rho_{b}}{\rho_{o}}\left(\frac{b}{\operatorname{Re}_{o} f_{R} R}\right)^{0.3}\left[\left(\frac{f_{R}}{f_{u}}\right)^{0.3}-1\right]} \frac{1}{z / d_{o}+z_{o} / d_{o}}
$$

from which the jet coefficient may be defined

$$
h_{c} \equiv \frac{c_{j}}{\beta_{c}} \sqrt{\frac{N f_{N}}{8 \psi_{2,1}} \frac{\rho_{o}}{\rho}} \sqrt{1-8\left(\frac{\pi}{4} C_{k}\right)^{17 / 20} \frac{1}{N f_{N}} \frac{\rho_{b}}{\rho_{o}}\left(\frac{b}{\operatorname{Re}_{o} f_{R} R}\right)^{0.3}\left[\left(\frac{f_{R}}{f_{u}}\right)^{0.3}-1\right]}
$$

This new expression suggests that increasing the standoff distance will decrease the scaled velocity. Again, the effect is small because $b / \operatorname{Re}_{0} f_{R} R$ remains much less than 1 , and $1 / N f_{N}$ and $\rho_{b} / \rho_{o}$ and $\left(f_{R} / f_{u}\right)^{0.3}-1$ are order 1. Because it is small, it will be neglected in the further derivations, though a simple correction factor remains sufficient. Notably, the nozzle Reynolds number lies in the denominator of the correction term.

\subsection{Upwell Velocity Profile in the Presence of Bulk Flow}

Bulk flow may affect the velocity profile of the central upwell. Bulk flow is induced either by vessel level rises due to the injection of large amounts of liquid into the vessel from the PJM tubes or by bulk particle settling. The former clearly induces an upward velocity parallel to the central upwell jet as evidenced by level rises observed experimentally. For the latter, if particles and fluid comprise a single phase of average density and the particles are uniformly distributed, then the fall of heavier particles and 
elevation of lighter liquid gives rise to a net downward mass average velocity. ${ }^{1}$ In either case, this gives rise to a compound jet. A compound jet comprises a central jet surrounded by fluid in motion with velocity $U_{1}$ parallel to the jet axis.

Defining the $U_{1}$ as positive in the upward vertical direction keeping the downward pointing settling velocity $u_{i}^{T}$ positive, $U_{1}$ may be approximated in terms of the PVF as

$$
U_{1}=\frac{P V F}{t_{D}} \frac{\left(H_{s}+\frac{4 V_{\text {head }}}{\pi D^{2}}\right)}{\left(1-\frac{N D_{P J M}^{2}}{D^{2}}\right)}-\sum_{i=1}^{n} w_{i} u_{i}^{T}\left(1-\frac{\rho_{f}}{\rho_{s, i}}\right)
$$

where $H_{s}$ is the height above the seam, $t_{D}$ is the drive time, $D_{P J M}$ is the diameter of the PJM tube, $w_{i}$ is the mass fraction in suspension, $u_{i}{ }^{T}$ is the magnitude of the settling velocity ${ }^{2}$ of particle $i$, and $\rho_{s, i}$ is the density of solids $i$. $V_{\text {head }}$ has been retained, generally because it varies from the spherical head in the $8 \mathrm{ft}$ vessel to a 2:1 elliptical head in the proposed $16 \mathrm{ft}$ vessel. Alternatively, $U_{1}$ may be approximated as

$$
U_{1}=\frac{\Delta l_{P J M}}{t_{D}\left(\frac{D^{2}}{N D_{P J M}^{2}}-1\right)}-\sum_{i=1}^{n} w_{i} u_{i}^{T}\left(1-\frac{\rho_{f}}{\rho_{s, i}}\right)
$$

where $\Delta l_{P J M}$ is the change in pulse tube level. These formulations conveniently lump bulk flow from both sources together. To estimate which of the two effects is larger, Table 3.2.1 of run sheet 7 (CCN 271190) gives drive times of 5.5 to $33 \mathrm{~s}$ for the range of nozzle velocities $(5.00$ to $15.00 \mathrm{~m} / \mathrm{s})$ and PVFs $(0.10$ to $0.20)$ of interest in the $8 \mathrm{ft}$ vessel and Figure $1.1 H_{s}=78.7$ inches $(2.00 \mathrm{~m})$ for $H / D=1$. Braun (2012) gives $D=92.5453$ inches, and BNI (2014) gives the PJM pipe as 16 inch Schedule 10S so that the outer diameter $D_{P J M}=16.000$ inches and the inner diameter $D_{P J M}=15.624$ inches. Then the bulk average velocity from a PJM pulse is 0.0015 to $0.044 \mathrm{~m} / \mathrm{s}$ from the first term on the right in Eq. 3.29 with $V_{\text {head }}=0$ in this estimate. Similarly, the most challenging simulant that does not have significant hindered settling is the full six-part simulant from Table 2.2. Table 3.2 shows that the contribution of particle settling is approximately $7.3 \cdot 10^{-4} \mathrm{~m} / \mathrm{s}$. This is approximately two orders of magnitude smaller than the velocity due to PJM level rise, suggesting that lumped particle settling may be safely neglected in this context (individual species may still settle as considered in Section 4.2).

\footnotetext{
${ }^{1}$ Solids move down at a rate of $u_{i}^{T}$ and a corresponding volume of displaced fluid moves up at the same net rate. Please note that this derivation does not differentiate between free and hindered settling and only considers the terminal velocity. Expressions for settling in a non-Newtonian fluid may also apply (see Kuhn et al. 2013, Appendix E), which is why the term is left somewhat general.

${ }^{2}$ The settling velocity is defined as positive giving the minus sign in this equation.
} 
Table 3.2. Bulk Mass Average Velocity Due to Particle Settling for Run Sheet $7^{(\mathrm{a})}$

\begin{tabular}{lcccc}
\hline Constituent & $\begin{array}{c}\text { Particle Density } \\
\left(\mathrm{g} / \mathrm{cm}^{3}\right)\end{array}$ & $\begin{array}{c}w_{i} \\
(\text { in suspension })\end{array}$ & $\begin{array}{c}u_{i}^{T} \\
(\mathrm{~m} / \mathrm{s})\end{array}$ & $\begin{array}{c}w_{i} u_{i}{ }^{T}\left(1-\rho_{f} / \rho_{s, i}\right) \\
(\mathrm{m} / \mathrm{s})\end{array}$ \\
\hline $\mathrm{SiC}$ & 3.2 & 0.0628 & $3.0 \cdot 10^{-5}$ & $1.3 \cdot 10^{-6}$ \\
$\mathrm{~W}^{(\mathrm{b})}$ & 9.6 & 0.0048 & $1.2 \cdot 10^{-3}$ & $5.0 \cdot 10^{-6}$ \\
$\mathrm{Glass}^{-3}$ powder & 2.5 & 0.0459 & $7.0 \cdot 10^{-3}$ & $1.9 \cdot 10^{-4}$ \\
$\mathrm{Al}_{2} \mathrm{O}_{3}$ & 3.9 & 0.0042 & $9.0 \cdot 10^{-2}$ & $2.8 \cdot 10^{-4}$ \\
$\mathrm{Sand}_{\text {Glass beads }}$ & 2.65 & 0.0012 & $1.3 \cdot 10^{-1}$ & $9.4 \cdot 10^{-5}$ \\
Sum & 2.5 & 0.0018 & $1.4 \cdot 10^{-1}$ & $1.5 \cdot 10^{-4}$ \\
\hline
\end{tabular}

(a) From Table 2.2. Suspension weight fractions based on 12.08\% from M. Minette email "MCE Loni testing through 10.doc" on 3/19/2015. FIO.

(b) $\mathrm{W}$ denotes the dense tungsten component, tungsten carbide with a density of $9.6 \mathrm{~g} / \mathrm{cm}^{3}$.

Following the arguments in the derivation for a compound jet (Rajaratnam 1976, Chapter 4) and preserving the constant flow $U_{1}$ in every derivative preserves the rate of excess momentum of the jet alone as

$$
2 \pi \rho \int_{0}^{\infty}\left(u-U_{1}\right)^{2} r d r
$$

(Rajaratnam's derivation limited integration from 0 to 2 in conjunction with the radial profile given by Squire and Trouncer, Eq. 2.6). Following Eq. 4-5 in Rajaratnam (1976), the velocity profile is asserted in the central upwell of the form

$$
u=U_{1}+\left(u_{m, c}-U_{1}\right) f_{c}\left(\eta_{c}\right)
$$

consistent with known jet physics to preserve similarity. ${ }^{1}$ When $\eta_{c}$ is large, $f_{c}$ vanishes and $u=U_{1}$ as expected. Conversely, when $\eta_{c}$ is small, $f_{c}$ approaches unity and $u=u_{m, c}$ as expected. Rajaratnam (1976) shows that momentum of compound jets is conserved. Therefore, setting the rate of momentum in Eq. (3.31) equal to the rate of momentum entering the control volume, $M_{o}$, given in Eqs. (3.1) to (3.3) gives

$$
2 \pi \delta_{c}^{2}\left(u_{m, c}-U_{1}\right)^{2} \rho \int_{0}^{\infty} f_{c}^{2}\left(\eta_{c}\right) \eta_{c} d \eta_{c}=M_{o}
$$

\footnotetext{
${ }^{1}$ Rajaratnam (1976) asserts that this only holds in the strong jet limit where $u_{m, c}>U_{1}$ but does not offer an alternative in the weak jet limit. The weak jet limit falls out of the derivation of Eq. 4-44 in Rajaratnam (1976), in which the momentum flux is $\rho u U_{1}$. This would lead to a scaling regime in which the jet velocity scales as $z^{-2 / 3}$ with a jet spread of $z^{1 / 3}$, although the functional form of the radial distribution of velocities was not given; see Rajaratnam (1976), Eq. 4-73. This would be advantageous because it would indicate that the jet would go further even though it is weaker, which is partially counterintuitive and partially justified by a lower spreading rate. However, Rajaratnam's Eqs. 4-44 and 3.31 herein differ only in how the constant $U_{1}$ is treated in derivatives, and the weak jet region only falls out if one arbitrarily forces the derivatives of the constant to zero. Furthermore, Rajaratnam (1976) is left to ponder in Chapter 4 thereof why experimental data for slow jets scales as $z^{-1}$, suggesting that the weak jet scaling regime may be fictitious. For the purposes of this report, this suggests that the self-similar profile may be reasonable for all ratios of $u_{m, c} / U_{1}$. In Rajaratnam's strong jet limit, the derivations herein become identical at his Eq. 4-49.
} 
Solving for $u_{m, c}$ gives

$$
u_{m, c}=U_{1}+\frac{c_{j}}{\beta_{c}} \sqrt{\frac{M_{o}}{2 \pi \rho \psi_{2,1} d_{o}^{2}}} \frac{d_{o}}{z+z_{o}}
$$

where $c_{j}$ represents an additional jet constant (if needed to fit data). ${ }^{1}$ More generally, Lo may be defined as the Loni number, which represents the ratio of the rate of jet momentum to that of the peripheral bulk fluid,

$$
L o^{2}=\frac{M_{o}}{\frac{\pi}{4} \rho d_{o}^{2} U_{1}^{2}}
$$

The Loni number is a member of the larger generalized family of Froude numbers that represent the ratio of inertia to an external field (there typically gravity) but is distinguished here because the traditional Froude numbers vary with geometric scale, but this number does not. Please note that the square of the Loni number was defined such that the Loni number must always be nonnegative regardless of the sign of $U_{1}$. Then,

$$
u_{m, c}=U_{1}+\frac{c_{j}}{\beta_{c}} \frac{U_{1} L o}{\sqrt{8 \psi_{2,1}}} \frac{d_{o}}{z+z_{o}}
$$

and in scaled terms,

$$
\frac{u_{m, c}}{U_{o}}=\frac{U_{1}}{U_{o}}+\frac{c_{j}}{\beta_{c}} \frac{L o}{\sqrt{8 \psi_{2,1}}} \frac{U_{1}}{U_{o}} \frac{d_{o}}{z+z_{o}}
$$

from which the following may be defined

$$
h_{c} \equiv \frac{c_{j}}{\beta_{c}} \frac{L o}{\sqrt{8 \psi_{2,1}}} \frac{U_{1}}{U_{o}}
$$

Substituting Eq. (3.1) gives

$$
L o=\sqrt{\frac{N f_{N} \rho_{o}}{\rho}} \frac{U_{o}}{U_{1}} \text { and } h_{c} \equiv \frac{c_{j}}{\beta_{c}} \sqrt{\frac{N f_{N}}{8 \psi_{2,1}} \frac{\rho_{o}}{\rho}}
$$

which is otherwise identical to Eq. (3.9). Substituting Eq. (3.2) gives

\footnotetext{
${ }^{1}$ Abramovich (1963, Chapter 1) argues that the radial spread of jets may be substantially influenced by co/counter current flow relative to the jet axis.
} 


$$
L o=\sqrt{\frac{16 N f_{N}}{\pi^{2}} \frac{\rho_{o}}{\rho}} \frac{Q}{d_{o}^{2} U_{1}} \text { and } h_{c} \equiv \frac{c_{j}}{\beta_{c}} \sqrt{\frac{2 N f_{N}}{\pi^{2} \psi_{2,1}} \frac{\rho_{o}}{\rho}} \frac{Q}{d_{o}^{2} U_{o}}
$$

Substituting Eq. (3.3) gives

$$
L o=\sqrt{\frac{16 f_{N}}{N \pi^{2}} \frac{\rho_{o}}{\rho}} \frac{V_{\text {vessel }} P V F}{d_{o}{ }^{2} t_{D} U_{1}} \text { and } h_{c} \equiv \frac{c_{j}}{\beta_{c}} \sqrt{\frac{2 f_{N}}{N \pi^{2} \psi_{2,1}} \frac{\rho_{o}}{\rho}} \frac{V_{\text {vessel }} P V F}{d_{o}{ }^{2} t_{D} U_{o}}
$$

These expressions show how $h_{c}$ may vary in the absence of geometric scaling with constant Strouhal number $\left(\mathrm{Sr}=t_{D} U_{o} / d_{o}\right)$ and thereby facilitates comparison of disparate data sets.

Finally, it is noted that the ratio of $U_{1} / U_{o}$ is typically small in the systems of relevance. As discussed above, $U_{1}=0.0015-0.044 \mathrm{~m} / \mathrm{s}$, and Table 2.3 shows $U_{o}=3.7-15 \mathrm{~m} / \mathrm{s}$. This suggests that bulk motion in these systems is relatively quiescent compared to the jet itself. Indeed, a jet that traverses only a few tens of millimeters each second will surely fail to reach the surface with drive times of only 5.5 to $33 \mathrm{~s}$. This simplifies the analysis considerably. Therefore, in the data analysis that follows the ratio of $U_{l} / U_{o}$ will be neglected.

\subsection{Jet Coefficients Compared to Data}

Having removed from consideration a prominent role for bulk motion relative to jet motion, the jet fitting constant, $c_{j}$, may now be determined. This is the final remaining factor in direct prediction of $h_{c}$ for a free jet without necessarily resorting to experiments. To estimate $c_{j}$, Eq. (3.9) may be rearranged to find

$$
c_{j}=\beta_{c} h_{c} \sqrt{\frac{8 \psi_{2,1}}{N f_{N}} \frac{\rho}{\rho_{o}}}
$$

The experimental and model predicted values of $h_{c}$ along with $c_{j}$ are found in Table 3.3. The agreement between prediction and experiment for water from Bontha et al. (2003a) is remarkably good. With the introduction of solids, the agreement diminishes with $c_{j}$ typically in the range of $1 / 3$ to $1 / 2$. Therefore, the model overpredicts the jet flow in the presence of solids by approximately a factor of two to three. Some of this momentum may contribute to fan jets that arise between PJM tubes instead of the central upwell, though the fan jets do not rise particularly high (see Figure 1.1). Some of the momentum is opposed by forces from the solids, including negative buoyancy and yield stresses. For an analytical model that does not directly account for the negative buoyancy of solids loadings to come within a factor of approximately three is remarkable. This is particularly true given the uncertainty in the value of $\beta_{c}$ that varies over a factor of three as seen in Table 2.1. Approximations of the jet curve in the presence of nonconservative forces (i.e., forces that dissipate momentum) that changes the flow regime are addressed in the following sections. 
Table 3.3. Central Upwell Jet Coefficients ${ }^{(a)}$

\begin{tabular}{|c|c|c|c|c|}
\hline Reference & Constituents & $\begin{array}{c}h_{c} \\
(\operatorname{expt})\end{array}$ & $\begin{array}{c}h_{c} \\
\text { (model) }\end{array}$ & $c_{j}$ \\
\hline Bontha et al. (2003a) & Water & 4.64 & 4.87 & 0.954 \\
\hline Bontha et al. (2003b) & Water & 2.64 & 5.11 & 0.517 \\
\hline Bontha et al. (2003b) & $10 \mu \mathrm{m}$ glass beads $5 \mathrm{wt} \%$ & 2.55 & 3.00 & 0.851 \\
\hline Bontha et al. (2003b) & $10 \mu \mathrm{m}$ glass beads $20 \mathrm{wt} \%$ & 2.48 & 5.02 & 0.494 \\
\hline Bontha et al. (2003b) & $35 \mu \mathrm{m}$ glass beads $5 \mathrm{wt} \%$ & 2.69 & 7.63 & 0.353 \\
\hline Bontha et al. (2003b) & $35 \mu \mathrm{m}$ glass beads $20 \mathrm{wt} \%$ & 2.46 & 8.39 & 0.293 \\
\hline Bamberger et al. (2005) & KB slurry, APEL, $U_{o}>10 \mathrm{~m} / \mathrm{s}$ & 1.5 & 10.17 & 0.147 \\
\hline Bamberger et al. (2005) & KB slurry, APEL, $U_{o}<10 \mathrm{~m} / \mathrm{s}$ & 0.209 & 10.17 & 0.0205 \\
\hline Bamberger et al. (2005) & KB slurry, LTTS, $U_{o}>7 \mathrm{~m} / \mathrm{s}$ & 2.07 & 10.17 & 0.204 \\
\hline Commissioning $^{(b)}$ & Water & 6.49 & 9.98 & 0.650 \\
\hline Run sheet $1^{(b)}$ & Glass powder, $\mathrm{Al}_{2} \mathrm{O}_{3}$ & 4.26 & 9.98 & 0.427 \\
\hline Run sheet $2^{(\mathrm{b})}$ & Glass powder, $\mathrm{Al}_{2} \mathrm{O}_{3}$ & 2.41 & 9.98 & 0.241 \\
\hline Run sheet $3^{(b)}$ & KB slurry, $U_{o} \sim 12 \mathrm{~m} / \mathrm{s}$ & 5.29 & 15.36 & 0.344 \\
\hline Run sheet $7^{(b)}$ & Glass powder, $1.27 \mathrm{wt} \%$ & 4.82 & 9.98 & 0.483 \\
\hline Run sheet $7^{(\mathrm{b})}$ & Glass powder, 2.54 wt $\%$ & 5.1 & 9.98 & 0.511 \\
\hline Run sheet $7^{(\mathrm{b})}$ & Glass powder, 4.59 wt $\%$ & 4.98 & 9.98 & 0.499 \\
\hline Run sheet $7^{(b)}$ & Glass powder, $\mathrm{W}^{(\mathrm{c})}$ & 4.14 & 9.98 & 0.415 \\
\hline Run sheet $7^{(\mathrm{b})}$ & Glass powder, ${ }^{\left({ }^{(c)}\right.} \mathrm{SiC}, U_{o} \geq 12.0 \mathrm{~m} / \mathrm{s}$ & 4.54 & 9.98 & 0.455 \\
\hline Run sheet $7^{(\mathrm{b})}$ & Glass powder, $\mathrm{W}^{\left({ }^{(c)}\right.} \mathrm{SiC}$, beads & 5.6 & 9.98 & 0.561 \\
\hline Run sheet $7^{(b)}$ & $\begin{array}{l}\text { Glass powder, W, }{ }^{(\mathrm{c})} \mathrm{SiC} \text {, beads, } \mathrm{Al}_{2} \mathrm{O}_{3} \text {, } \\
\text { sand, } U_{0} \geq 12.0 \mathrm{~m} / \mathrm{s}\end{array}$ & 4.35 & 9.98 & 0.436 \\
\hline
\end{tabular}

(a) Assumes $N f_{N}=1$ for the $12.75 \mathrm{ft}$ vessel with four PJMs and $N f_{N}=2$ for the $8 \mathrm{ft}$ vessel with six PJMs and $\rho / \rho_{o}=1$. Because $\beta_{c}$ cannot be quantified from the velocity probes in the $8 \mathrm{ft}$ vessel, the value of $\beta_{c}$ is assumed to be 0.0848 for Newtonian rheologies and 0.0551 for KB slurries unless reported in Table 2.1. The value of $\psi_{2,1}=0.349$ from the Tollmien solution (Rajaratnam 1976) unless specified otherwise in Table 3.1.

(b) FIO.

(c) $\mathrm{W}$ denotes the dense tungsten component, tungsten carbide with a density of $9.6 \mathrm{~g} / \mathrm{cm}^{3}$.

As a purely empirical discovery, Figure 3.1 suggests that there may be a linear relationship between $z_{o} / d_{o}$ and $h_{c}$. Indeed, the linear correlation is remarkable for the $8 \mathrm{ft}$ test data for a variety of simulants including the two-part simulant of run sheets 1 and 2 and the various combinations of the multipart simulant from one to six components in water. The correlation also appears to hold within a factor of three for other vessel sizes, though it remains unclear whether other vessel sizes require separate linear fits. Additional experimental analysis of scalability and additional modeling is required to assert confidence in the extent to which these trends hold across scales and changes in vessel curvature. In any case, the virtual origin appears to deepen (increase in magnitude) as the jet coefficient increases and elevate (become smaller) as the jet coefficient decreases. Remarkably, this purely empirical trend appears to hold whether a distinct cloud height is present or not, though further analysis is required based on the models formulated in Section 4 and analyzed in Section 5. 


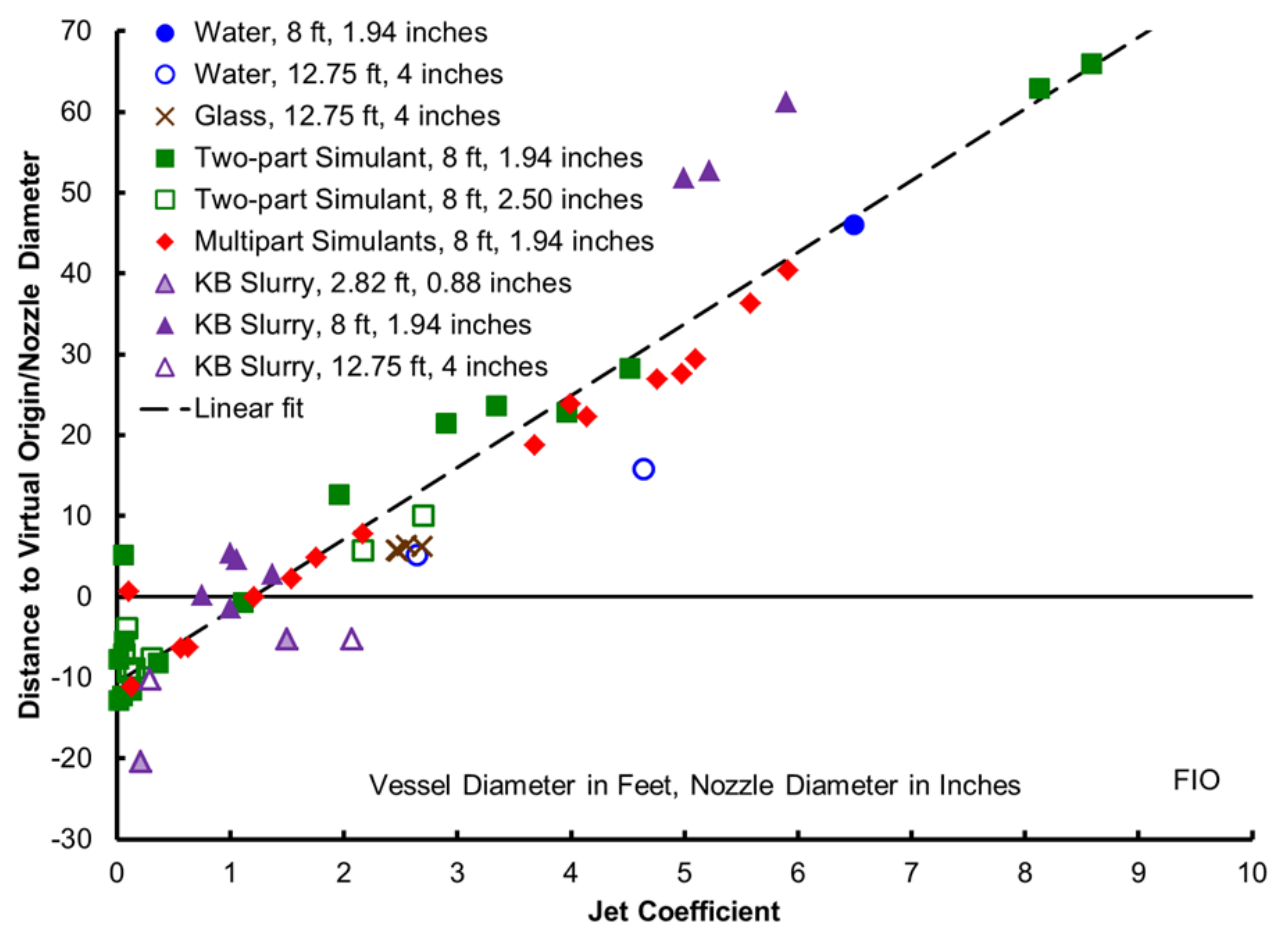

Figure 3.1. Depth of Virtual Origin Scaled on Nozzle Diameter Versus Jet Coefficient for a Variety of Vessels, Nozzle Diameters, and Simulants

\subsection{Summary}

The jet coefficient may be predicted from conservation of mass for a free jet when the radial distribution of velocities is known [Eq. (3.9)]. Shear stress along the bottom and flows parallel to the jet axis only marginally affect the magnitude of the jet coefficient [e.g., Eqs. (3.18) and (3.28)]. The depth of the virtual origin scaled on nozzle diameter appears to depend approximately linearly on the jet coefficient, though this result should be considered preliminary because there is insufficient analyses to confirm that this correlation does not contain an implicit scale dependence. Model predictions based on conservation of momentum alone only hold for a free jet and overpredict the jet coefficient by a factor of approximately 2-3 (Table 3.3) for Newtonian simulants. Though the jet coefficient for a Newtonian solids laden jet may be approximated by dividing the model jet coefficient by a factor of three, Section 5 provides a more appropriate expression for the jet coefficient. Predicted jet coefficients should not be used for yield stress fluids; see Section 6 for better velocity profiles as a function of elevation in yield stress materials. 



\subsection{Framework for Evaluating Alternative Flow Regimes}

An essential feature of scaling from one vessel to the next is the requirement that the underlying physics does not change. Indeed, one may have confidence in scaling if 1) the correlations capture the relevant physics; 2) all factors relevant to scaling have been incorporated, including flow, material, and geometric considerations; 3 ) the uncertainty in the relationships is sufficiently narrow to meet required specifications, and perhaps most importantly; 4) the underlying physics does not change from the conditions under which the correlations were developed to the condition(s) of interest. The last is particularly critical. Indeed, while available data convincingly show the central upwell to be a free jet in many cases, available data also indicate that the underlying physics does change with lower jet momentum and the flow does deviate from the proposed free jet profile for the central upwell.

This section presents a framework for considering alternative flow regimes that may have yield and buoyancy forces both influential simultaneously. Sections 5 through 7 describe specific flow regimes that curtail the central free jet, benchmarks them against experimental data, and evaluates their scalability.

\subsection{Conservation of Momentum Including Yield and Buoyancy}

Several forces may influence transitions from the free jet regime to other competing flow regimes. This section returns to species and momentum conservation to evaluate the influence of viscous, yield, negative buoyancy, and transient effects together in a single framework. In so doing, it allows for direct comparison of these effects and holds simultaneously for both Newtonian and non-Newtonian fluids, even when the adverse features of both are influential simultaneously.

While negative buoyancy (where material falls instead of rises) can be included comprehensively in nonlinear computational fluid dynamics (CFD) code, ${ }^{1}$ this effect is typically included analytically by using the Boussinesq approximation (Deen 2012; Crapper and Baines 1976). This approximation selects a reference density, $\rho_{\infty}$ (e.g., the density of fluid far from the center of the jet), rewrites the dynamic pressure, $\wp$, relative to this reference density such that a buoyancy term naturally arises, and then asserts that all other variations in density (e.g., in the continuity equation or in the generalized expressions for the deviatoric stress tensor) may simply be replaced by the reference density (Deen 2012). The density, $\rho$, relative to the reference density in the buoyancy term may then be expanded in terms of thermal or compositional variations.

Current WTP simulants usually have three to seven species (e.g., two- to six-part simulants in water). Yet, previous derivations using the Boussinesq approximation for negative buoyancy have focused almost exclusively on thermally driven fountains, where the heat and mass transfer analogy provides limited physical insight into multiphase flows. Formulating the buoyancy term precisely is important because the Froude number (a ratio of inertia to gravity) falls out of this analysis and may differ meaningfully from prior definitions.

Defining the dynamic pressure as $\nabla \wp=\nabla P-\rho_{\infty} \boldsymbol{g}$ and inserting it into Cauchy's momentum equation (the precursor to the classical Navier-Stokes equation) gives

\footnotetext{
${ }^{1}$ Many CFD codes linearize the buoyancy term when nondimensionalized on a densimetric Froude number, which may not accurately represent the nonlinear influence of this term.
} 


$$
\rho_{\infty} \frac{D \boldsymbol{v}}{D t}=-\nabla \wp+\left(\rho-\rho_{\infty}\right) \boldsymbol{g}+\nabla \cdot \boldsymbol{\tau}
$$

where $\boldsymbol{v}$ is the velocity vector, $P$ is the pressure, script $\wp$ is the dynamic pressure, $\boldsymbol{g}$ is the downward pointing gravitational vector, and $\tau$ is the deviatoric stress tensor (Deen 2012). The density may be approximated in a Taylor series expansion of which the first terms are

$$
\rho=\rho_{\infty}+\sum_{i} \frac{\partial \rho}{\partial w_{i}}\left(w_{i}-w_{i}^{\infty}\right)
$$

where $w_{i}$ is the weight fraction of species $i$ and $w_{i}^{\infty}$ is the corresponding reference weight fraction of species $i$, presumably far from the center of the jet. A solutal coefficient may be defined in the usual form (Deen 2012) as

$$
\beta_{i} \equiv-\frac{1}{\rho_{\infty}} \frac{\partial \rho}{\partial w_{i}}
$$

The values of the solutal coefficient may be positive or negative and this formulation allows for contributions from multiple species.

Substitution of the solutal coefficient into Cauchy's momentum equation yields

$$
\rho_{\infty} \frac{D \boldsymbol{v}}{D t}=-\nabla \wp-\rho_{\infty} \boldsymbol{g} \sum_{i} \beta_{i}\left(w_{i}-w_{i}^{\infty}\right)+\nabla \cdot \boldsymbol{\tau}
$$

To simplify the negative buoyancy term, the slurry density may be written in terms of the volume fractions $\phi_{i}$ and species particle densities $\rho_{i}$ as $\rho=\Sigma_{i} \phi_{i} \rho_{i}$. From masses $m_{i}$, the volume fractions may be constructed via volumes $V_{i}=m_{i} / \rho_{i}$ as $\phi_{i}=\left(w_{i} / \rho_{i}\right) / \Sigma_{j} w_{j} / \rho_{j}$ for thermodynamically ideal solutions by dividing through by the total mass. Then,

$$
\rho=\left(\sum_{j} \frac{w_{j}}{\rho_{j}}\right)^{-1}
$$

because $\Sigma_{i} w_{i}=1$. Taking the derivative of the former gives

$$
\frac{\partial \rho}{\partial w_{i}}=-\rho^{2}\left(\frac{1}{\rho_{i}}+\sum_{j \neq i} \frac{1}{\rho_{j}} \frac{\partial w_{j}}{\partial w_{i}}\right)
$$

after back-substituting the definition of density. It may be shown by induction and a limit proof that

$$
\frac{\partial w_{j}}{\partial w_{i}}=-\frac{w_{j}}{1-w_{i}}
$$


for $i \neq j$. This expression reduces succinctly to the binary case where taking the derivative of $w_{1}+w_{2}=1$ with respect to $w_{1}$ gives the well-known result of $d w_{2} / d w_{1}=-1$. Substitution then gives the solutal coefficient generally as

$$
\beta_{i}=\frac{\rho^{2}}{\rho_{\infty}}\left(\frac{1}{\rho_{i}}-\sum_{j \neq i} \frac{1}{\rho_{j}} \frac{w_{j}}{1-w_{i}}\right)
$$

Adding and subtracting the term on the right for species $i$ after factoring the $1-w_{\mathrm{i}}$ out simplifies this equation to

$$
\beta_{i}=\frac{1}{1-w_{i}} \frac{\rho^{2}}{\rho_{\infty}}\left(\frac{1}{\rho_{i}}-\frac{1}{\rho}\right)
$$

The buoyancy term then becomes

$$
-\rho_{\infty} \boldsymbol{g} \sum_{i} \beta_{i}\left(w_{i}-w_{i}^{\infty}\right)=-\rho_{\infty} \boldsymbol{g} \frac{\rho^{2}}{\rho_{\infty}} \sum_{i} \frac{w_{i}-w_{i}^{\infty}}{1-w_{i}}\left(\frac{1}{\rho_{i}}-\frac{1}{\rho}\right)
$$

In the spirit of the Boussinesq approximation, a Taylor series expansion gives

$$
\frac{1}{1-w_{i}}=1+w_{i}+w_{i}^{2}+\ldots
$$

In the dilute species limit $w_{i}<<1$, so that only the first term in the expansion is retained as higher order terms introduce nonlinearity. The buoyancy term then becomes

$$
-\rho_{\infty} \boldsymbol{g} \sum_{i} \beta_{i}\left(w_{i}-w_{i}^{\infty}\right)=-\boldsymbol{g} \rho^{2} \sum_{i}\left(w_{i}-w_{i}^{\infty}\right)\left(\frac{1}{\rho_{i}}-\frac{1}{\rho}\right)
$$

which may be simplified as

$$
-\rho_{\infty} \boldsymbol{g} \sum_{i} \beta_{i}\left(w_{i}-w_{i}^{\infty}\right)=-\boldsymbol{g} \rho^{2}\left(\frac{1}{\rho}-\frac{1}{\rho_{\infty}}\right)=\boldsymbol{g} \rho\left(\frac{\rho-\rho_{\infty}}{\rho_{\infty}}\right)
$$

by repeated application of Eq. (4.5) following distribution. ${ }^{1}$

\footnotetext{
${ }^{1}$ Admittedly, water itself is not dilute, which adds a term of

$$
\boldsymbol{g} \frac{\rho}{\rho_{w}}\left(\rho-\rho_{w}\right) w_{w} \frac{w_{w}^{\infty}-w_{w}}{1-w_{w}}
$$

to Eqs. 4.12, 4.13, and 4.14, where the subscript $w$ denotes water alone. Although order 1, this term will not be considered further herein due to its nonlinearity.
} 
Reynolds averaging for turbulence in the axial direction in the absence of swirl (i.e., $v_{\theta}=0$ ) gives

$$
\rho_{\infty} \frac{D \boldsymbol{v}}{D t}=-\nabla \wp+\boldsymbol{g} \rho\left(\frac{\rho-\rho_{\infty}}{\rho_{\infty}}\right)+\nabla \cdot\left(\boldsymbol{\tau}_{\text {lam }}+\boldsymbol{\tau}_{\text {turb }}\right)
$$

where the terms now represent suitable temporal averages (Deen 2012), $u$ and $v$ represent scalar velocities in the axial and radial directions, respectively, and the usual deviatoric viscous stress, $\tau_{\text {lam }}$, now has a turbulent counterpart in the Reynolds stress, $\boldsymbol{\tau}_{\text {turb }}$. The corresponding expression in the radial direction becomes uninteresting after an initial startup time that is shorter than that in the axial direction by a factor of $\beta_{c}^{2}$.

There are two analytically straightforward models for the turbulent stresses, one attributed to Prandtl and the other to Boussinesq and Prandtl (Bird et al. 2002; Abramovich 1963). Both are evaluated herein. First, for Prandtl's mixing length theorem, the axial component of the stress tensors can be written out for a Bingham plastic using the Bingham-Papanastasiou formulation (Mitsoulis 2007) with Prandtl's mixing length theory as

$$
\tau_{\text {lam }}+\tau_{\text {turb }}=\tau_{o}\left(1-\operatorname{Exp}\left[-m\left|\frac{\partial u}{\partial r}\right|\right]\right)+\mu_{o} \frac{\partial u}{\partial r}+\rho_{\infty} l^{2}\left|\frac{\partial u}{\partial r}\right| \frac{\partial u}{\partial r}
$$

where the terms on the right represent stresses from the yield, viscous, and turbulent stresses (Rajaratnam 1976; Bird et al. 2002). This derivation asserts the working assumption of the existence of a physical yield stress at modest shear stresses even though this construct has neither been proven nor modestly explored (see footnote in Section 2.2). With the traditional Prandtl mixing length, $l=C\left(z+z_{o}\right)$, where $C^{2}$ is a constant equal to $\left(\beta_{c} / 1.24\right)^{3}$ from Rajaratnam (1976, pp. 37-38). Section 4.3 derives relationships between $\beta_{c}$ and $C$ from first principles for a free jet. The Bingham-Papanastasiou formulation allows for continuous differentiation of the shear stress and determination of the solid-fluid boundaries a posteriori. The variable $m$ is a regularization parameter with units of time (see Figure 2.6); the ideal Bingham plastic formulation is recovered as $m$ goes to infinity as will be done hereafter. Substitution then gives

$$
\begin{aligned}
\rho_{\infty} \frac{\partial u}{\partial t}+\rho_{\infty} v \frac{\partial u}{\partial r}+\rho_{\infty} u \frac{\partial u}{\partial z}=-\frac{\partial \wp}{\partial z} & -g \rho\left(\frac{\left.\rho-\rho_{\infty}\right)}{\rho_{\infty}}\right)+\frac{\tau_{o}}{r} \\
& +\mu_{o} \frac{1}{r} \frac{\partial}{\partial r}\left(r \frac{\partial u}{\partial r}\right)+\rho_{\infty} \frac{C^{2}}{r} \frac{\partial}{\partial r}\left[r\left(z+z_{o}\right)^{2}\left(\frac{\partial u}{\partial r}\right)^{2}\right]
\end{aligned}
$$

after expanding the velocity vectors on the left hand side. The minus sign on the negative buoyancy term arises because the coordinate system points up in opposition to gravity. This is the governing momentum equation that includes both negative buoyancy and yield stresses.

This equation may now be scaled with the axial velocity on $U_{o}$, the axial length on $d_{o}$, the radial length on $\beta_{c} d_{o}$, the radial velocity on $\beta_{c} U_{o}$ from continuity, the time on the drive time, $t_{D}$, the pressure on $\rho_{\infty} U_{o}^{2}$, and the local slurry density on $\rho_{\infty}$. This gives 


$$
\begin{aligned}
\frac{d_{o}}{t_{D} U_{o}} \frac{\partial \bar{u}}{\partial \bar{t}}+\bar{v} \frac{\partial \bar{u}}{\partial \bar{r}}+\bar{u} \frac{\partial \bar{u}}{\partial \bar{z}} & =-\frac{\partial \bar{\wp}}{\partial \bar{z}}-\frac{g d_{o}}{U_{o}^{2}} \bar{\rho}(\bar{\rho}-1)+\frac{\tau_{o}}{\beta_{c} \rho_{\infty} U_{o}^{2}} \frac{1}{\bar{r}} \\
& +\frac{\mu_{o}}{\beta_{c}{ }^{2} \rho_{\infty} U_{o} d_{o}} \frac{1}{\bar{r}} \frac{\partial}{\partial \bar{r}}\left(\bar{r} \frac{\partial \bar{u}}{\partial \bar{r}}\right)+\frac{C^{2}}{\beta_{c}^{3}} \frac{1}{\bar{r}} \frac{\partial}{\partial \bar{r}}\left[\bar{r}\left(\bar{z}+\bar{z}_{o}\right)^{2}\left(\frac{\partial \bar{u}}{\partial \bar{r}}\right)^{2}\right]
\end{aligned}
$$

where the $O(1)$ terms for the free jet have been retained and overbars represent scaled values. These can be written out in terms of dimensionless numbers as

$$
\begin{aligned}
\frac{1}{\operatorname{Sr}} \frac{\partial \bar{u}}{\partial \bar{t}}+\bar{v} \frac{\partial \bar{u}}{\partial \bar{r}}+\bar{u} \frac{\partial \bar{u}}{\partial \bar{z}}= & -\frac{\partial \bar{\wp}}{\partial \bar{z}}-\frac{1}{\operatorname{Fr}} \bar{\rho}(\bar{\rho}-1)+\frac{1}{\beta_{c} \operatorname{Re}_{\tau}} \frac{\rho_{o}}{\rho_{\infty}} \frac{1}{\bar{r}} \\
& +\frac{1}{\beta_{c}{ }^{2} \operatorname{Re}_{o}} \frac{\rho_{o}}{\rho_{\infty}} \frac{1}{\bar{r}} \frac{\partial}{\partial \bar{r}}\left(\bar{r} \frac{\partial \bar{u}}{\partial \bar{r}}\right)+\frac{C^{2}}{\beta_{c}{ }^{3}} \frac{1}{\bar{r}} \frac{\partial}{\partial \bar{r}}\left[\bar{r}\left(\bar{z}+\bar{z}_{o}\right)^{2}\left(\frac{\partial \bar{u}}{\partial \bar{r}}\right)^{2}\right]
\end{aligned}
$$

where

$$
\begin{gathered}
\mathrm{Sr}=\frac{t_{D} U_{o}}{d_{o}} \\
\operatorname{Re}_{\tau}=\frac{\rho_{o} U_{o}{ }^{2}}{\tau_{o}} \\
\operatorname{Re}_{o}=\frac{\rho_{o} U_{o} d_{o}}{\mu_{o}}
\end{gathered}
$$

and

$$
\mathrm{Fr}=\frac{U_{o}{ }^{2}}{g d_{o}}
$$

Some may prefer the square root of this number as the Froude number.

This definition of the Froude number differs significantly from others proposed in the literature. For example, Kuhn et al. (2013) conservatively base the Froude number on the tank diameter as $\mathrm{Fr}=U_{o}{ }^{2} /[(s-1) g D]$, where $s$ is a density ratio (Section 2.2.2.4, Chapter 5 and Appendix C). This makes sense if the vessel is mixed by impellers, wall jets, or possibly even for a planar jet that spans the vessel, because in each of these cases, a characteristic length is the vessel diameter. However, the central jet does not inherently interact with the vessel walls nor does it necessarily sense the vessel height, particularly if the jet changes regime before reaching the upper surface. Therefore, basing the Froude 
number on the nozzle diameter for the central upwell is not only appropriate but necessary. ${ }^{1}$ With geometric scaling, both $D$ and $d_{o}$ change in parallel, so one might argue that this is a difference without a distinction. However, changing a nozzle diameter remains more straightforward than changing vessel diameter, and this formulation provides that flexibility.

Perhaps more intriguing is the role of density in the Froude number. Literature models suggest the inclusion of a factor of $(s-1)$ in the denominator of the Froude number (see Kuhn et al. 2013). This development shows, however, that the appropriate term in the denominator would be $s(s-1)$, if included. Furthermore, this definition of Froude number without including density follows the time honored tradition of keeping scales distinct from independent variables unless absolutely necessary and is in excellent agreement with the definitions in Deen (2012, p. 264) and Bird et al. (2002, p. 98) that also do not include densities. This Froude number does not vary with axial position and does not change if the dilute species limit does not hold.

An analytically tractable alternative to Prandtl's mixing length is Boussinesq's eddy viscosity model, also attributed to Prandtl or Goertler in the context of jet physics (Bird et al. 2002; Rajaratnam 1976). Here, the dynamic turbulent viscosity is proportional to the product of the reference density, width of the mixing zone, and the velocity along the centerline of the jet. ${ }^{2}$ Because $u_{m, c}$ is inversely proportional to $z$ and $\delta_{c}$ is proportional to $z$, this viscosity is independent of spatial position like the usual dynamic viscosity. ${ }^{3}$ Then the deviatoric stresses in the axial direction become

$$
\tau_{\text {lam }}+\tau_{\text {turb }}=\tau_{o}\left(1-\operatorname{Exp}\left[-m\left|\frac{\partial u}{\partial r}\right|\right]\right)+\mu_{o} \frac{\partial u}{\partial r}+\rho_{\infty} C_{2} h_{c} U_{o} d_{o} \frac{\partial u}{\partial r}
$$

with substitution of Eq. (2.1). Rajaratnam (1976, pp. 41 and 45) gives $C_{2}=18.5^{-2}$. Section 4.3 derives relationships between $\beta_{c}$ and $C_{2}$ for free jets. For typical values of the jet, the turbulent viscosity (e.g., the last coefficient in Eq. $4.23 \rho_{\infty} C_{2} h_{c} U_{o} d_{o} \geq 0.59 \mathrm{~Pa}$ s for $\rho_{\infty}=1000, h_{c} \geq 1, U_{o} \geq 4 \mathrm{~m} / \mathrm{s}$, and $d_{o}=2$ inches) exceeds the molecular viscosity (e.g., $\leq 0.030 \mathrm{~Pa}$ s), but both terms will be retained through scaling. Substitution then gives

$$
\rho_{\infty} \frac{\partial u}{\partial t}+\rho_{\infty} v \frac{\partial u}{\partial r}+\rho_{\infty} u \frac{\partial u}{\partial z}=-\frac{\partial \wp}{\partial z}-g \rho\left(\frac{\rho-\rho_{\infty}}{\rho_{\infty}}\right)+\frac{\tau_{o}}{r}+\left(\mu_{o}+\rho_{\infty} C_{2} h_{c} U_{o} d_{o}\right) \frac{1}{r} \frac{\partial}{\partial r}\left(r \frac{\partial u}{\partial r}\right)
$$

This is the governing momentum equation that includes both negative buoyancy and yield stresses.

This equation may be scaled similarly on the axial velocity on $U_{o}$, the axial length on $d_{o}$, the radial length on $\beta_{c} d_{o}$, the radial velocity on $\beta_{c} U_{o}$ from continuity, the time on the drive time, $t_{D}$, the pressure on $\rho_{\infty} U_{o}^{2}$, and the local slurry density on $\rho_{\infty}$. This gives

\footnotetext{
${ }^{1}$ The radial outward flow along the top of the vessel that moves out to the vessel wall (in the absence of jet attachment or crossflow) has the vessel radius as a characteristic length. However, the scope of this report is limited to the upwell portion of the flow. Velocity probes that would measure radial flow across the fluid surface have not been implemented historically or in the $8 \mathrm{ft}$ vessel.

${ }^{2}$ This form assumes peripheral velocities to be much smaller than the centerline velocity.

${ }^{3}$ Except perhaps near a cloud height or jet front where $u_{m, c}$ must decay more rapidly than $\sim z^{-1}$ (Shekarriz et al. 1995b). A stagnation point arises in these cases, the conceptual equivalent of which is flow approximately normal to a flat plate (see Example 9.4-2 in Deen 2012).
} 


$$
\begin{aligned}
\frac{d_{o}}{t_{D} U_{o}} \frac{\partial \bar{u}}{\partial \bar{t}}+\bar{v} \frac{\partial \bar{u}}{\partial \bar{r}}+\bar{u} \frac{\partial \bar{u}}{\partial \bar{z}}=- & \frac{\partial \bar{\wp}}{\partial \bar{z}}-\frac{g d_{o}}{U_{o}{ }^{2}} \bar{\rho}(\bar{\rho}-1) \\
& +\frac{\tau_{o}}{\beta_{c} \rho_{\infty} U_{o}{ }^{2}} \frac{1}{\bar{r}}+\frac{\mu_{o}+\rho_{\infty} C_{2} h_{c} U_{o} d_{o}}{\beta_{c}{ }^{2} \rho_{\infty} U_{o} d_{o}} \frac{1}{\bar{r}} \frac{\partial}{\partial \bar{r}}\left(\bar{r} \frac{\partial \bar{u}}{\partial \bar{r}}\right)
\end{aligned}
$$

where the order 1 terms for the free jet have been retained. These may be written out in terms of dimensionless numbers defined previously as

$$
\begin{aligned}
\frac{1}{S r} \frac{\partial \bar{u}}{\partial \bar{t}}+\bar{v} \frac{\partial \bar{u}}{\partial \bar{r}}+\bar{u} \frac{\partial \bar{u}}{\partial \bar{z}}= & -\frac{\partial \bar{\wp}}{\partial \bar{z}}-\frac{1}{F r} \bar{\rho}(\bar{\rho}-1) \\
& +\frac{1}{\beta_{c} \operatorname{Re}_{\tau}} \frac{\rho_{o}}{\rho_{\infty}} \frac{1}{\bar{r}}+\left(\frac{1}{\beta_{c}^{2} \operatorname{Re}_{o}} \frac{\rho_{o}}{\rho_{\infty}}+\frac{C_{2} h_{c}}{\beta_{c}{ }^{2}}\right) \frac{1}{\bar{r}} \frac{\partial}{\partial \bar{r}}\left(\bar{r} \frac{\partial \bar{u}}{\partial \bar{r}}\right)
\end{aligned}
$$

The terms in Eqs. (4.18) and (4.26) are evaluated in the remainder of this section. Please note that geometric scale differences are embedded completely in the four dimensionless groups. Only if these are scale dependent will the jet flow be scale dependent. (Additional scale-dependent parameters may also arise in boundary or initial conditions.)

\subsection{Particle Profile Within the Upwell}

The governing momentum equations show that the buoyancy term depends on the species concentration profile, coupling species conservation with momentum. Therefore, this multiphase analysis evaluates species conservation to determine how the weight fraction varies as a function of spatial position in the presence and absence of a cloud height.

Where $q$ species (including the fluid) are present and continuity is used, $q-1$ independent species balances may be constructed. For example, two particles of different sizes or densities in a fluid would constitute $q=3$ species, so two species mass balances may be constructed independently. (The weight fraction of the remaining species derives from $\Sigma_{i} w_{i}=1$.) The mass flux of particles of species or size $i, \boldsymbol{j}_{\boldsymbol{i}}$, crossing an arbitrary control surface is the sum of convective and diffusive terms,

$$
\boldsymbol{j}_{i}=\boldsymbol{v} w_{i}-D_{d i f f} \nabla w_{i}
$$

where $w_{i}$ and $D_{\text {diff }}$ represent the mass fraction and diffusivities of species $i$, respectively. The mass average velocity vector, $v$, here contains both the axial and radial velocities described below as well as species-specific particle settling velocity (Kuhn et al. 2013). The diffusivity is the sum of the molecular, $D_{i}$, and turbulent, $D_{t}$, diffusivities. Then the mass flux in the axial direction becomes

$$
j_{i, z}=\left(u-u_{i}^{T}\right) w_{i}-\left(D_{i}+D_{t}\right) \frac{\partial w_{i}}{\partial z}
$$

Within the jet, the molecular diffusivity is much smaller than the turbulent diffusivity such that it can be neglected in the remainder of the derivation (Rao 1980). Conservation of mass requires 


$$
\frac{\partial w_{i}}{\partial t}=-\nabla \cdot j_{i}
$$

in the absence of particle formation or degradation (possible with agglomeration/aggregation or the opposite). ${ }^{1}$

To determine the influence of the settling velocity in this context (a distinct question from its influence on momentum), the particle flux may be set to zero at the cloud height at steady state. In the absence of turbulent diffusion, the cloud height may occur where the settling velocity and upwell velocity $\left(u=u_{i}{ }^{T}\right)$ balance in the absence of negative buoyancy effects. Then with Eq. (2.1)

$$
\frac{z_{\text {cloud }}}{d_{o}}=\frac{h_{c} U_{o}}{u_{i}^{T}}-\frac{z_{o}}{d_{o}}
$$

where $z_{\text {cloud }}$ is the cloud height. This expression shows that the cloud height increases with the nozzle velocity and also suggests that cloud height would increase with nozzle diameter and decrease with settling velocity, though there is no substantial evidence to suggest distinct cloud heights for each species present due to multiphase jet flow during a pulse. (Residual clouds from the prior pulse may remain as a distinct cloud height from that discussed here.) Quantitatively, however, Eq. (4.30) remains insufficient. For example, consider the two part simulant, which displayed instances with and without surface flow. The simulants have unhindered settling velocities of $6.2 .10^{-3}$ to $7.2 \cdot 10^{-2} \mathrm{~m} / \mathrm{s}$ (Table 2.2), and $h_{c}=4.26$ and $z_{o} / d_{o}=32.8$ from Table 2.4. For the range of velocities considered, $U_{o}=6.21-12.03 \mathrm{~m} / \mathrm{s}, z_{\text {cloud }} / d_{o}>300$, which vastly exceeds the dimensions of the vessel $\left(z / d_{o}=48\right.$, quiescent) even though a finite cloud height was observed. Similarly, for the particle with the largest settling velocity, the glass beads, $u_{i}{ }^{T}=0.14 \mathrm{~m} / \mathrm{s}$, $h_{c}=5.40$, and $z_{o} / d_{o}=36.3$, giving $z_{\text {cloud }} / d_{o}>200$. Therefore, in the absence of accelerated settling, which Kuhn et al. (2013, Section C.4.2.10) argue for in Hanford wastes, ${ }^{2}$ individual particle settling may be safely ignored relative to the centerline velocity during the drive portion of the pulse. Hindered settling typically decreases the particle settling rate, further strengthening these arguments.

When axial turbulence is not negligible at steady state, setting the species flux to zero yields

$$
u=u_{i}{ }^{T}+D_{t} \frac{\partial \ln w_{i}}{\partial z}
$$

The turbulent diffusivity is related to the turbulent viscosity through the turbulent Schmidt number, $\mathrm{Sc}_{t}=v_{t} / D_{t}$. Bird et al. (2002) assert $\mathrm{Sc}_{t}=0.7$ specifically for circular free jets, while Bajpai and Tirumkudulu (2008) assert $\mathrm{Sc}_{t}=30$. With the turbulent viscosity from Prandtl's mixing length theory, the turbulent diffusivity becomes

\footnotetext{
${ }^{1}$ For some simulants, agglomeration clearly happens on relevant time scales such that the reaction term neglected here may be important in certain settings. The simulant prepared by Carolyne Burns of PNNL for the $8 \mathrm{ft}$ vessel testing does not show significant time-dependent hysteresis (Figure 2.6) such that this mechanism is negligible for this simulant.

${ }^{2}$ The settling behavior of multicomponent Hanford wastes remains a compelling but unsolved problem, for which no complete analytical solution is available to improve the accuracy and precision of this analysis. This is at least partially true because the interparticle attractive and repulse forces have not been quantified.
} 


$$
D_{t}=\frac{v_{t}}{S c_{t}}=\frac{l^{2}}{S c_{t}}\left|\frac{\partial u}{\partial r}\right|
$$

Taylor and Krishna (1993) argue that the turbulent diffusivity is species independent because it is a flow property not a molecular property. ${ }^{1}$ Substituting in Eqs. (2.1) and (2.3) with the Gaussian profile from Eq. (2.5) yields

$$
D_{t}=\frac{2 \operatorname{Ln}(2) C^{2} h_{c} U_{o} d_{o}}{S c_{t} \beta_{c}} \eta_{c} \operatorname{Exp}\left[-\operatorname{Ln}(2) \eta_{c}^{2}\right]
$$

With Boussinesq's eddy viscosity model

$$
D_{t}=\frac{v_{t}}{S c_{t}}=\frac{C_{2} h_{c} U_{o} d_{o}}{S c_{t}}
$$

Since the exact functional form of the species concentration profile in the vicinity of the cloud height remains unknown, ${ }^{2}$ but experimental observations suggest a distinct density difference above and below the cloud height, a Heaviside step function in the weight fraction may be approximated with a Fermi-Dirac function so that

$$
w_{i}=w_{i}^{\max }\left(1+\operatorname{Exp}\left[\frac{z-z_{\text {cloud }}}{s_{\text {cloud }}}\right]\right)^{-1}
$$

where $w_{i}^{\max }$ is the concentration at the base of the jet, $z_{\text {cloud }}$ is the cloud height, and $s_{\text {cloud }}$ characterizes the slope of the concentration profile at $z_{\text {cloud }}$. The derivative of the natural $\log$ of this expression gives

$$
\frac{d \ln w_{i}}{d z}=-\frac{\operatorname{Exp}\left[\frac{z-z_{\text {cloud }}}{s_{\text {cloud }}}\right]}{s_{\text {cloud }}\left(1+\operatorname{Exp}\left[\frac{z-z_{\text {cloud }}}{s_{\text {cloud }}}\right]\right)}
$$

Therefore, evaluating this derivative at the cloud height with Boussinesq's eddy viscosity model gives

$$
D_{t} \frac{\partial \ln w_{i}}{\partial z} \sim-\frac{C_{2} h_{c} U_{o} d_{o}}{2 S c_{t} S_{\text {cloud }}}
$$

\footnotetext{
${ }^{1}$ This form of the diffusivity assumes the thermodynamics and mechanics of particle interaction are negligible, but such terms could be included to advance consideration of concentrated suspensions with suitable expressions for the chemical potential of the slurry.

${ }^{2}$ A sigmoidal curve may fall out of the shock condition associated with the jet penetration front described in Section 7 in the presence of a negative buoyancy force.
} 
With $C_{2}=18.5^{-2}, h_{c}=1-6, U_{o}=3-15 \mathrm{~m} / \mathrm{s}, \mathrm{Sc}_{t}=0.7-30$, and $s_{\text {cloud }} / d_{o}=1$ (based on visual observation of the experiments), the second term in Eq. 4.31 ranges from $1.5 \cdot 10^{-4}$ to $0.19 \mathrm{~m} / \mathrm{s}$, which is typically larger than that of the glass powder in Table 2.2. Additionally, the term has a sign that opposes the settling velocity, further confirming that the settling velocity does not drive cloud height formation. This does not imply that the cloud does not depend on particle properties (the cloud height clearly does depend on particle derived properties, see Section 5).

With settling velocity eliminated as a key player even in the cloud height, the complete expression for species conservation may be constructed. Substitution of Eq. (4.27) into Eq. (4.29) followed by Reynolds averaging to account for turbulence gives

$$
\frac{\partial w_{i}}{\partial t}+u \frac{\partial w_{i}}{\partial z}+v \frac{\partial w_{i}}{\partial r}=\frac{\partial}{\partial z}\left(D_{t} \frac{\partial w_{i}}{\partial z}\right)+\frac{1}{r} \frac{\partial}{\partial r}\left(r D_{t} \frac{\partial w_{i}}{\partial r}\right)
$$

With Boussinesq's eddy viscosity model, the turbulent diffusivity in the absence of negative buoyancy gives

$$
\frac{\partial w_{i}}{\partial t}+u \frac{\partial w_{i}}{\partial z}+v \frac{\partial w_{i}}{\partial r}=D_{t} \frac{\partial}{\partial z}\left(\frac{\partial w_{i}}{\partial z}\right)+D_{t} \frac{1}{r} \frac{\partial}{\partial r}\left(r \frac{\partial w_{i}}{\partial r}\right)
$$

Scaling the axial velocity on $U_{o}$, the axial length on $d_{o}$, the radial length on $\beta_{c} d_{o}$, the radial velocity on $\beta_{c} U_{o}$ from continuity, and the drive time on $t_{D}$ then gives

$$
\frac{1}{S r} \frac{\partial w_{i}}{\partial \bar{t}}+\bar{u} \frac{\partial w_{i}}{\partial \bar{z}}+\bar{v} \frac{\partial w_{i}}{\partial \bar{r}}=\frac{\beta_{c}^{2}}{P e} \frac{\partial}{\partial \bar{z}}\left(\frac{\partial w_{i}}{\partial \bar{z}}\right)+\frac{1}{P e} \frac{1}{\bar{r}} \frac{\partial}{\partial \bar{r}}\left(\bar{r} \frac{\partial w_{i}}{\partial \bar{r}}\right)
$$

This is the governing species conservation equation. This equation and Eq. (4.26) form a coupled pair that may be solved simultaneously to predict the fluid and particle profiles within the vessel. Because $\beta_{c}<1$, the radial term exceeds the axial term and the coefficient of the radial term is recognized as a Peclet number where

$$
P e \equiv \frac{\beta_{c}^{2} U_{o} d_{o}}{D_{t}}=\frac{\beta_{c}{ }^{2} S c_{t}}{C_{2} h_{c}}
$$

with substitution of Eq. (4.34). Numerically, $P e$ ranges considerably around order one from 0.11 to 244, with $\beta_{c}=0.0551-0.154, C_{2}=18.5^{-2}, S c_{t}=0.7$ to 30 , and $h_{c}=1$ to 6.5 from Table 2.1 and Table 2.4 . As the radial spread of the jet increases as measured by $\beta_{c}$, the radial concentration gradients diminish and the right-hand side of the species concentration equation becomes less important. The implication is that both turbulent diffusion and turbulent convection are of similar magnitude.

Four insights emerge from this development in the absence of a significant settling velocity. First, with the assumption that the particle is in the upwell jet flow created during the drive portion of the PJM cycle, the time scale for particle flow is exactly the same as the time scale for jet flow. As a result, there is not a separate particle time scale during the drive portion of the PJM duty cycle within the upwell. 
Second, in the absence of a compelling settling velocity argument as described above, none of the coefficients in Eq. (4.40) depend on species specific parameters. Therefore, none of the dimensionless groups formed by them will be species specific, and, due to the linearity in $w_{i}$ of Eq. (4.40) and its Prandtl mixing length equivalent, scaled concentration profiles will also not depend on the species. Therefore, all of the particles in the upwell follow the same time averaged and scaled flow profiles (see Section 5.2). (These profiles are, however, influenced by the aggregate or lumped properties from species present; see Section 5.2.) This affirms the assumption of a single solids fraction and a single concentration gradient by Kuhn et al. (2013, Eq. C.96).

Third, this analysis, therefore, requires that cloud height ${ }^{1}$ formation to the extent it arises from the central upwell be governed by negative buoyancy considerations alone. Only when the fluid dynamics are limiting such that the other terms in the flux vanish may the settling velocity act as a restoring force with its time scale. This implies that observations of $\mathrm{SiC}$ and $\mathrm{W}$ particles above the cloud height in the six-part experiments were the result of suspension at higher velocities prior to the beginning of a test with incomplete settling between tests and do not comprise a separate cloud height in the sense that the cloud height has been used in historical testing (Meyer et al. 2012) and run sheets 1, 2, and 6 in $8 \mathrm{ft}$ testing.

Fourth, and most importantly, none of the terms in this governing equation are geometric scale dependent. Behavior at one vessel size will be the same in another geometrically scaled vessel in the absence of additional constraints through the initial and boundary conditions. Analysis in Section 5 suggests that the initial and boundary conditions may incorporate a geometric scale dependence through fluid dynamics considerations.

None of these insights implies or is meant to imply that if the particle's settling velocity is large enough to oppose the turbulent jet flow that it will not settle out or separate from the flow (e.g., gravel would clearly settle out). Clearly far from the jet centerline, the turbulent jet flow decays and particle settling dominates as it does when the jet flow is finished. Furthermore, only particles carried into the upwell (an initial condition to these equations) can be transported by the upwell in the manner described; particles that never enter the upwell cannot be transported by the upwell. Other flows including the wall jet (shown to be weak in Section 8) or radial wall jets along the vessel bottom may have their own distinctive "cloud heights." Finally, the cloud height must be a function of particle properties as they influence the initial conditions and fluid dynamics of the upwell jets (see Section 5).

Perhaps the most positive aspect of the absence of a species-specific terminal settling rate is the opportunity to use the heat and mass transfer analogy. Bird et al. (2002) solved the steady state temperature profile for a free turbulent jet. Simplifying the governing species conservation equation for steady state, imposing $\beta_{c}<<1$ (always in Table 2.1), removing the scaling, and letting

\footnotetext{
${ }^{1}$ This analysis clarifies the term "cloud height" associated with multicomponent multiphase flow, which has previously been unclear. A single cloud height may be an intuitively clear experimental observable in the presence of a single monodispersed simulant in an otherwise clear solvent. With two or more types of particles in water, one may question whether there is one cloud height for the mixture or one for each solid species. This analysis argues that if the particle settling velocities are substantially smaller than the fluid velocities then there is only one cloud height for all of the species, not several cloud heights for the several species individually. (If some settling velocities are so large that the particles never leave the neighborhood of the vessel bottom or reach the upwell, the remainder may still have a single cloud height.) Please note that in run sheets 1 and 2, only one cloud height was observed for the two-part simulant in water to which both particles rose. Particles may be above the hydrodynamically defined cloud height from prior runs and that may also be termed a "cloud," but that is not the cloud defined herein.
} 


$$
\Theta \equiv \frac{w_{i}-w_{i}^{\infty}}{w_{i}^{\max }-w_{i}^{\infty}}
$$

where $w_{i}^{\max }$ and $w_{i}^{\infty}$ are the mass fraction of species $i$ at the base of the jet and far from the center of the jet, respectively, gives

$$
u \frac{\partial \Theta_{i}}{\partial z}+v \frac{\partial \Theta_{i}}{\partial r}=\frac{v_{t}}{S c_{t}} \frac{1}{r} \frac{\partial}{\partial r}\left(r \frac{\partial \Theta_{i}}{\partial r}\right)
$$

This equation is identical to Eq. 13.5-4 in Bird et al. (2002), with the substitution of $S c_{t}$ for $P r_{t}$ as expected of the heat and mass transfer analogy. Asserting that $w_{i}=w_{i}^{\max }$ at $z=0$ and that $w_{i}$ is finite at the centerline of the jet but decays to $w_{i}^{\infty}$ as $r$ approaches infinity, the corresponding boundary conditions are

$$
\Theta=1 \text { at } z=0
$$

$\Theta$ is finite at $r=0$

$$
\Theta=0 \text { as } r \rightarrow \infty
$$

completing the set of necessary equations and alignment with the Eqs. 13.5-5 through 13.5-7 of Bird et al. (2002). The radial distribution of velocities with the functional form of Eq. (2.8) falls naturally out of their solution and the radial distribution of the particle concentration profile becomes

$$
\frac{\Theta}{\Theta_{\max }}=\left(\frac{u}{u_{m, c}}\right)^{S c_{t}}
$$

where $\Theta_{\max }$ is the concentration profile along the jet centerline. This may be simplified in our notation as

$$
w_{i}=\left(w_{i}^{c}-w_{i}^{\infty}\right) f_{c}^{S c_{t}}+w_{i}^{\infty}
$$

This is commensurate with the work of Melville and Bray (1979a, b), Dawson and Trauss (1966), and Rao (1980), who assert that the concentration profiles in circular and radial jets, like the jet profiles themselves, are self-similar, albeit slightly more narrow as expected of $S c_{t}<1$. Remarkably, the solution of Bird et al. (2002) also provides that the concentration profile decays inversely with elevation. The constant of proportionality is anchored to the selection of $w_{i}^{\max }$ via the boundary condition Eq. (4.44), giving

$$
w_{i}^{c}=\frac{z_{o}}{z+z_{o}}\left(w_{i}^{\max }-w_{i}^{\infty}\right)+w_{i}^{\infty}
$$

The implication of this $\sim 1 / z$ decay in weight fraction is that the density will also decay with a similar proportionality and asymptote out to the bulk density at higher elevations. 
The radial density profile may be obtained from the species concentration profile by dividing it through by the solid species density $\rho_{i}$ and summing over all species. Implementing Eq. (4.5) gives

$$
\frac{1}{\rho}=\left(\frac{1}{\rho_{c}}-\frac{1}{\rho_{\infty}}\right) f_{c}^{S c_{t}}+\frac{1}{\rho_{\infty}}
$$

Though the functional form may be unanticipated, it may be inverted as

$$
\rho=\frac{\rho_{c} \rho_{\infty}}{\left(\rho_{\infty}-\rho_{c}\right) f_{c}^{S c_{t}}+\rho_{c}}
$$

and gives the appropriate limits for $f_{c}=0$ and 1 . Similarly, the axial density profile along the centerline is

$$
\frac{1}{\rho_{c}}=\frac{z_{o}}{z+z_{o}}\left(\frac{1}{\rho_{\max }}-\frac{1}{\rho_{\infty}}\right)+\frac{1}{\rho_{\infty}}
$$

This may also be inverted to give

$$
\rho_{c}=\frac{\rho_{\max } \rho_{\infty}}{\frac{z_{o}}{z+z_{o}}\left(\rho_{\infty}-\rho_{\max }\right)+\rho_{\max }}
$$

These expressions feed into an approximation of the cloud height in Section 5. Additional derivation and analysis given in Section 5.2.

\subsection{Coupled Energy Balances}

Energy balances may be coupled with momentum balances. For example, Eqs. (4.16) and (4.24) in the absence of dominating pressure gradients, buoyancy, yield stresses, viscous stresses, and transients may be multiplied by $u r d r$ and integrated over the domain of $r$ to find

$$
\begin{aligned}
& \int_{0}^{\infty} \rho_{\infty} v \frac{\partial u}{\partial r} u r d r+\int_{0}^{\infty} \rho_{\infty} u \frac{\partial u}{\partial z} u r d r=\int_{0}^{\infty} \rho_{\infty} \frac{C^{2}}{r} \frac{\partial}{\partial r}\left[r\left(z+z_{o}\right)^{2}\left(\frac{\partial u}{\partial r}\right)^{2}\right] u r d r \\
& \int_{0}^{\infty} \rho_{\infty} v \frac{\partial u}{\partial r} u r d r+\int_{0}^{\infty} \rho_{\infty} u \frac{\partial u}{\partial z} u r d r=\int_{0}^{\infty}\left(\rho_{\infty} C_{2} h_{c} U_{o} d_{o}\right) \frac{1}{r} \frac{\partial}{\partial r}\left(r \frac{\partial u}{\partial r}\right) u r d r
\end{aligned}
$$

respectively. Following Rajaratnam (1976, Chapter 2.5), integration by parts with combination of the first two terms on the right yields 


$$
\begin{gathered}
\left.\rho_{\infty} \frac{1}{2} r v u^{2}\right|_{0} ^{\infty}+\rho_{\infty} \frac{1}{2} \frac{d}{d z} \int_{0}^{\infty} u^{3} r d r=\left.\rho_{\infty} C^{2} u r\left(z+z_{o}\right)^{2}\left(\frac{\partial u}{\partial r}\right)^{2}\right|_{0} ^{\infty}-\rho_{\infty} C^{2} \int_{0}^{\infty} r\left(z+z_{o}\right)^{2}\left(\frac{\partial u}{\partial r}\right)^{2} \frac{\partial u}{\partial r} d r \\
\left.\rho_{\infty} \frac{1}{2} r v u^{2}\right|_{0} ^{\infty}+\rho_{\infty} \frac{1}{2} \frac{d}{d z} \int_{0}^{\infty} u^{3} r d r=\left.u r\left(\rho_{\infty} C_{2} h_{c} U_{o} d_{o}\right) \frac{\partial u}{\partial r}\right|_{0} ^{\infty}-\int_{0}^{\infty} r\left(\rho_{\infty} C_{2} h_{c} U_{o} d_{o}\right) \frac{\partial u}{\partial r} \frac{\partial u}{\partial r} d r
\end{gathered}
$$

For the free jet, the first terms on both sides vanish because the axial velocity goes to zero infinitely far from the jet centerline for a free jet, leaving

$$
\begin{aligned}
& \frac{1}{2} \frac{d}{d z} \int_{0}^{\infty} u^{3} r d r=-C^{2}\left(z+z_{o}\right)^{2} \int_{0}^{\infty} r\left(\frac{\partial u}{\partial r}\right)^{3} d r \\
& \frac{1}{2} \frac{d}{d z} \int_{0}^{\infty} u^{3} r d r=-C_{2} h_{c} U_{o} d_{o} \int_{0}^{\infty}\left(\frac{\partial u}{\partial r}\right)^{2} r d r
\end{aligned}
$$

The left-hand sides represent the energy in the jet and the right-hand sides represent the entropic losses to the jet. For the free jet, the momentum balance allows imposition of similarity and assertion of $\eta_{c}=r / \delta_{c}$ to find

$$
\begin{aligned}
& \frac{1}{2} \frac{d}{d z}\left(u_{m, c}^{3} \delta_{c}^{2} \int_{0}^{\infty} f_{c}^{3} \eta_{c} d \eta_{c}\right)=-C^{2}\left(z+z_{o}\right)^{2} \frac{u_{m, c}}{\delta_{c}} \int_{0}^{\infty} \eta_{c}\left(\frac{\partial f_{c}}{\partial \eta_{c}}\right)^{3} d \eta_{c} \\
& \frac{1}{2} \frac{d}{d z}\left(u_{m, c}^{3} \delta_{c}^{2} \int_{0}^{\infty} f_{c}^{3} \eta_{c} d \eta_{c}\right)=-C_{2} h_{c} U_{o} d_{o} u_{m, c}{ }^{2} \int_{0}^{\infty}\left(\frac{\partial f_{c}}{\partial \eta_{c}}\right)^{2} \eta_{c} d \eta_{c}
\end{aligned}
$$

The integrals are constants independent of $z$ and may be defined as

$$
\psi_{3,1}=\int_{0}^{\infty} f_{c}^{3} \eta_{c} d \eta_{c} \quad \psi_{0,1,3}=\left|\int_{0}^{\infty} \eta_{c}\left(\frac{\partial f_{c}}{\partial \eta_{c}}\right)^{3} d \eta_{c}\right| \quad \psi_{0,1,2}=\int_{0}^{\infty} \eta_{c}\left(\frac{\partial f_{c}}{\partial \eta_{c}}\right)^{2} d \eta_{c}
$$

leaving

$$
\begin{gathered}
\frac{d}{d z}\left(u_{m, c}{ }^{3} \delta_{c}^{2}\right)=-2 C^{2}\left(z+z_{o}\right)^{2} \frac{u_{m, c}{ }^{3}}{\delta_{c}} \frac{\psi_{0,1,3}}{\psi_{3,1}} \\
\frac{d}{d z}\left(u_{m, c}{ }^{3} \delta_{c}^{2}\right)=-\frac{2 C_{2} h_{c} U_{o} d_{o} \psi_{0,1,2}}{\psi_{3,1}} u_{m, c}{ }^{2}
\end{gathered}
$$


Table 4.1 gives the values of these constants for the theoretical and experimental profiles in Section 2.2. The final column shows that for the Tollmien solution, $\left(2 \psi_{0,1,3} / \psi_{3,1}\right)^{1 / 3}=1.24$ as asserted by Rajaratnam (1976); see Eq. (4.15).

For a circular free jet, Rajaratnam (1976, Chapter 2) argues for proportionalities to $z$, which Rao (1980) gives as $u_{m, c}=A /\left(z+z_{o}\right)$ and $\delta_{c}=\beta_{c}\left(z+z_{o}\right)$ with the addition of a virtual origin. Modifying the derivative with a constant then gives

$$
\begin{gathered}
\frac{\partial}{\partial\left(z+z_{o}\right)}\left(\frac{A^{3} \beta_{c}^{2}}{z+z_{o}}\right)=-\frac{2 C^{2}}{\left(z+z_{o}\right)^{2}} \frac{A^{3}}{\beta_{c}} \frac{\psi_{0,1,3}}{\psi_{3,1}} \\
\frac{\partial}{\partial\left(z+z_{o}\right)}\left(\frac{A^{3} \beta_{c}^{2}}{z+z_{o}}\right)=-\frac{2 C_{2} A \psi_{0,1,2}}{\psi_{3,1}} \frac{A^{2}}{\left(z+z_{o}\right)^{2}}
\end{gathered}
$$

which simplify to

$$
\begin{aligned}
& \beta_{c}{ }^{3}=2 C^{2} \frac{\psi_{0,1,3}}{\psi_{3,1}} \\
& \beta_{c}{ }^{2}=\frac{2 C_{2} \psi_{0,1,2}}{\psi_{3,1}}
\end{aligned}
$$

recognizing from Eq. (2.1) that $A=h_{c} U_{o} d_{o}$. This shows that the relationship between $\beta_{c}$ and $C$ as given below Eq. (4.15) depends directly on the radial profiles. Remarkably, this derivation also shows that the momentum and energy balances effectively decouple, so that the results of the momentum balance feed sequentially into the energy balance. For a free jet, the selection of a turbulence model only affects the value of $\beta_{c}$ of the jet spreading rate.

Table 4.1. Constants Derived from Radial Distributions

\begin{tabular}{llccccc}
\hline Reference & Model or Constituent & $\psi_{3,1}$ & $\psi_{1,0}$ & $\psi_{0,1,2}$ & $\psi_{0,1,3}$ & $\left(2 \psi_{0,1,3} / \psi_{3,1}\right)^{1 / 3}$ \\
\hline Wiegel (1964) & Gaussian (Eq. (2.5)) & 0.240 & 1.06 & 0.500 & 0.284 & 1.33 \\
Rajaratnam (1976) & Squire and Trouncer (Eq. (2.6)) & 0.242 & 1.00 & 0.617 & 0.411 & 1.50 \\
Rajaratnam (1976) & Prandtl/Tollmien (Eq. (2.7)) & 0.215 & 1.05 & 0.433 & 0.206 & 1.24 \\
Bird et al. (2002) & Boussinesq/Goertler (Eq. (2.8)) & 0.241 & 1.22 & 0.400 & 0.195 & 1.17 \\
Bontha et al. (2003b) & Water & 0.257 & 1.09 & 0.516 & 0.298 & 1.32 \\
Bontha et al. (2003b) & $10 \mu \mathrm{m}$ glass beads 5 wt\% & 0.257 & 1.08 & 0.532 & 0.320 & 1.36 \\
Bontha et al. (2003b) & $10 \mu \mathrm{m}$ glass beads 20 wt $\%$ & 0.267 & 1.03 & 0.672 & 0.530 & 1.58 \\
Bontha et al. (2003b) & $35 \mu \mathrm{m}$ glass beads 5 wt\% & 0.288 & 1.01 & 0.822 & 0.804 & 1.77 \\
Bontha et al. (2003b) & $35 \mu \mathrm{m}$ glass beads 20 wt\% & 0.259 & 1.10 & 0.501 & 0.279 & 1.29 \\
Bamberger et al. (2005) & KB slurry & 0.342 & 1.00 & 1.284 & 1.977 & 2.26 \\
\hline
\end{tabular}

However, in the presence of a yield stress, the energy balance becomes more interesting. For example, Eqs. (4.16) and (4.24) in the absence of dominating pressure gradients, buoyancy, and viscous 
stresses but retaining the yield stresses may be multiplied by $u r d r$ and integrated over the domain of $r$ to find

$$
\begin{aligned}
& \int_{0}^{R_{j e t}(z)} \rho_{\infty} v \frac{\partial u}{\partial r} u r d r+\int_{0}^{R_{j e t}(z)} \rho_{\infty} u \frac{\partial u}{\partial z} u r d r=\int_{0}^{R_{j e t}(z)} \frac{\tau_{o}}{r} u r d r+\int_{0}^{R_{j e t}(z)} \rho_{\infty} \frac{C^{2}}{r} \frac{\partial}{\partial r}\left[r\left(z+z_{o}\right)^{2}\left(\frac{\partial u}{\partial r}\right)^{2}\right] u r d r \\
& \int_{0}^{R_{j e t}(z)} \rho_{\infty} v \frac{\partial u}{\partial r} u r d r+\int_{0}^{R_{j e t}(z)} \rho_{\infty} u \frac{\partial u}{\partial z} u r d r=\int_{0}^{R_{j e t}(z)} \frac{\tau_{o}}{r} u r d r+\int_{0}^{R_{j e t}(z)}\left(\rho_{\infty} C_{2} h_{c} U_{o} d_{o}\right) \frac{1}{r} \frac{\partial}{\partial r}\left(r \frac{\partial u}{\partial r}\right) u r d r
\end{aligned}
$$

Following Rajaratnam (1976, Chapter 2.5), integration by parts with combination of the first two terms on the right yields

$$
\begin{aligned}
\left.\rho_{\infty} \frac{1}{2} r v u^{2}\right|_{0} ^{R_{j e t}(z)}+\rho_{\infty} \frac{1}{2} \frac{d}{d z} \int_{0}^{R_{j e t}(z)} u^{3} r d r= & \left.\rho_{\infty} C^{2} u r\left(z+z_{o}\right)^{2}\left(\frac{\partial u}{\partial r}\right)^{2}\right|_{0} ^{R_{j e t}(z)}+\tau_{o} \int_{0}^{R_{j e t}(z)} u d r \\
& -\rho_{\infty} C^{2} \int_{0}^{R_{j e t}(z)} r\left(z+z_{o}\right)^{2}\left(\frac{\partial u}{\partial r}\right)^{3} d r \\
\left.\rho_{\infty} \frac{1}{2} r v u^{2}\right|_{0} ^{R_{j e t}(z)}+\rho_{\infty} \frac{1}{2} \frac{d}{d z} \int_{0}^{R_{j e t}(z)} u^{3} r d r=\left.u r\left(\rho_{\infty} C_{2} h_{c} U_{o} d_{o}\right) \frac{\partial u}{\partial r}\right|_{0} ^{R_{j e t}(z)}+\tau_{o} \int_{0}^{R_{j e t}(z)} u d r & -\int_{0}^{R_{j e t}(z)} r\left(\rho_{\infty} C_{2} h_{c} U_{o} d_{o}\right) \frac{\partial u}{\partial r} \frac{\partial u}{\partial r} d r
\end{aligned}
$$

The terms evaluated at $r=0$ disappear because $r$ is a factor, and terms evaluated at $r=R_{\text {jet }}(z)$ disappear because either the jet is only marginally confined such that $u$ decays sufficiently to zero far from the centerline or an approximate no slip boundary condition allows $u \sim 0$ at $R_{j e t}$ in the absence of bulk motion of the adjacent Bingham solid (see Section 6). Under these approximations,

$$
\begin{aligned}
& \frac{1}{2} \frac{d}{d z} \int_{0}^{R_{j e t}(z)} u^{3} r d r=\frac{\tau_{o}}{\rho_{\infty}} \int_{0}^{R_{j e t}(z)} u d r-C^{2}\left(z+z_{o}\right)^{2} \int_{0}^{R_{j e t}(z)} r\left(\frac{\partial u}{\partial r}\right)^{3} d r \\
& \frac{1}{2} \frac{d}{d z} \int_{0}^{R_{j e t}(z)} u^{3} r d r=\frac{\tau_{o}}{\rho_{\infty}} \int_{0}^{R_{j e t}(z)} u d r-C_{2} h_{c} U_{o} d_{o} \int_{0}^{R_{j e t}(z)}\left(\frac{\partial u}{\partial r}\right)^{2} r d r
\end{aligned}
$$

The left-hand sides represent the energy in the jet and the right-hand sides represent the entropic losses to the jet due to yield stresses and turbulence, respectively. Data analyzed to date (Figure 2.4) support the assertion of similarity with $\eta_{c}=r / \delta_{c}$ to give

$$
\frac{1}{2} \frac{d}{d z}\left(u_{m, c}{ }^{3} \delta_{c}{ }^{2} \int_{0}^{R_{j e t}(z)} f_{c}^{3} \eta_{c} d \eta_{c}\right)=\frac{\tau_{o}}{\rho_{\infty}} u_{m, c} \delta_{c} \int_{0}^{R_{j e t}(z)} f_{c} d \eta_{c}-C^{2}\left(z+z_{o}\right)^{2} \frac{u_{m, c}{ }^{3}}{\delta_{c}} \int_{0}^{R_{j e t}(z)} \eta_{c}\left(\frac{\partial f_{c}}{\partial \eta_{c}}\right)^{3} d \eta_{c}
$$




$$
\frac{1}{2} \frac{d}{d z}\left(u_{m, c}{ }^{3} \delta_{c}{ }^{2} \int_{0}^{R_{j e t}(z)} f_{c}^{3} \eta_{c} d \eta_{c}\right)=\frac{\tau_{o}}{\rho_{\infty}} u_{m, c} \delta_{c} \int_{0}^{R_{j e t}(z)} f_{c} d \eta_{c}-C_{2} h_{c} U_{o} d_{o} u_{m, c}{ }^{2} \int_{0}^{R_{j e t}(z)}\left(\frac{\partial f_{c}}{\partial \eta_{c}}\right)^{2} \eta_{c} d \eta_{c}
$$

An additional integral may be defined as

$$
\psi_{1,0}=\int_{0}^{\infty} f_{c} d \eta_{c}
$$

to first order approximation, leaving

$$
\begin{gathered}
\frac{d}{d z}\left(u_{m, c}{ }^{3} \delta_{c}{ }^{2}\right)=2 \frac{\tau_{o}}{\rho_{\infty}} u_{m, c} \delta_{c} \frac{\psi_{1,0}}{\psi_{3,1}}-2 C^{2}\left(z+z_{o}\right)^{2} \frac{u_{m, c}{ }^{3}}{\delta_{c}} \frac{\psi_{0,1,3}}{\psi_{3,1}} \\
\frac{d}{d z}\left(u_{m, c}{ }^{3} \delta_{c}^{2}\right)=2 \frac{\tau_{o}}{\rho_{\infty}} u_{m, c} \delta_{c} \frac{\psi_{1,0}}{\psi_{3,1}}-\frac{2 C_{2} h_{c} U_{o} d_{o} \psi_{0,1,2}}{\psi_{3,1}} u_{m, c}{ }^{2}
\end{gathered}
$$

A complete and proper solution of this equation requires coupling with momentum balances. Similarly simplifying Eqs. (4.16) and (4.24), multiplying by $r d r$ and integrating over the domain of $r$ gives

$$
\begin{aligned}
& \int_{0}^{R_{j e t}(z)} \rho_{\infty} v \frac{\partial u}{\partial r} r d r+\int_{0}^{R_{j e t}(z)} \rho_{\infty} u \frac{\partial u}{\partial z} r d r=\int_{0}^{R_{j e t}(z)} \frac{\tau_{o}}{r} r d r+\int_{0}^{R_{j e t}(z)} \rho_{\infty} \frac{C^{2}}{r} \frac{\partial}{\partial r}\left[r\left(z+z_{o}\right)^{2}\left(\frac{\partial u}{\partial r}\right)^{2}\right] r d r \\
& \int_{0}^{R_{j e t}(z)} \rho_{\infty} v \frac{\partial u}{\partial r} r d r+\int_{0}^{R_{j e t}(z)} \rho_{\infty} u \frac{\partial u}{\partial z} r d r=\int_{0}^{R_{j e t}(z)} \frac{\tau_{o}}{r} r d r+\int_{0}^{R_{j e t}(z)}\left(\rho_{\infty} C_{2} h_{c} U_{o} d_{o}\right) \frac{1}{r} \frac{\partial}{\partial r}\left(r \frac{\partial u}{\partial r}\right) r d r
\end{aligned}
$$

Following Rajaratnam (1976, Chapter 2.5), integration by parts with combination of the first two terms on the right yields

$$
\begin{gathered}
\left.\rho_{\infty} r v u\right|_{0} ^{R_{j e t}(z)}+\rho_{\infty} \frac{d}{d z} \int_{0}^{R_{j e t}(z)} u^{2} r d r=\left.\rho_{\infty} C^{2} r\left(z+z_{o}\right)^{2}\left(\frac{\partial u}{\partial r}\right)^{2}\right|_{0} ^{R_{j e t}(z)}+\tau_{o} \int_{0}^{R_{j e t}(z)} d r \\
\left.\rho_{\infty} r v u\right|_{0} ^{R_{j e t}(z)}+\rho_{\infty} \frac{d}{d z} \int_{0}^{R_{j e t}(z)} u^{2} r d r=\left.\left(\rho_{\infty} C_{2} h_{c} U_{o} d\right) r \frac{\partial u}{\partial r}\right|_{0} ^{R_{j e t}(z)}+\tau_{o} \int_{0}^{R_{j e t}(z)} d r
\end{gathered}
$$

The first terms vanish for the same reasons as lead to Eq. (4.64), leaving

$$
\rho_{\infty} \frac{d}{d z} \int_{0}^{R_{j e t}(z)} u^{2} r d r=\tau_{o} R_{j e t}(z)
$$


in both cases. Imposing similarity gives

$$
\frac{d}{d z}\left(u_{m, c}^{2} \delta_{c}^{2}\right)=\frac{\tau_{o} R_{j e t}(z)}{\rho_{\infty} \psi_{2,1}}
$$

Equations (4.67) and (4.71) form a coupled set that must be solved simultaneously.

To explore the consequences of the energy balance in the presence of a yield stress on the solution, consider for a moment allowing $u_{m, c}=A /\left(z+z_{o}\right)$ and $\delta_{c}=\beta_{c}\left(z+z_{o}\right)$, where hypothetically $A$ and $\beta_{c}$ are ersatz constants independent of $z$. Eq. (4.67b) gives

$$
\begin{gathered}
\frac{d}{d\left(z+z_{o}\right)}\left(\frac{A^{3} \beta_{c}{ }^{2}}{z+z_{o}}\right)=2 \frac{\tau_{o}}{\rho_{\infty}} A \beta_{c} \frac{\psi_{1,0}}{\psi_{3,1}}-\frac{2 C^{2}}{\left(z+z_{o}\right)^{2}} \frac{A^{3}}{\beta_{c}} \frac{\psi_{0,1,3}}{\psi_{3,1}} \\
\frac{d}{d\left(z+z_{o}\right)}\left(\frac{A^{3} \beta_{c}{ }^{2}}{z+z_{o}}\right)=2 \frac{\tau_{o}}{\rho_{\infty}} A \beta_{c} \frac{\psi_{1,0}}{\psi_{3,1}}-\frac{2 C_{2} h_{c} U_{o} d_{o} \psi_{0,1,2}}{\psi_{3,1}} \frac{A^{2}}{\left(z+z_{o}\right)^{2}}
\end{gathered}
$$

Evaluating the derivatives and simplifying gives

$$
\begin{gathered}
\beta_{c}{ }^{3}+2 \frac{\tau_{o}}{\rho_{\infty}} \frac{\beta_{c}{ }^{2}}{A^{2}} \frac{\psi_{1,0}}{\psi_{3,1}}\left(z+z_{o}\right)^{2}-2 C^{2} \frac{\psi_{0,1,3}}{\psi_{3,1}}=0 \\
\frac{1}{2} \beta_{c}^{2}+\frac{\tau_{o}}{\rho_{\infty}} \frac{\beta_{c}}{A^{2}} \frac{\psi_{1,0}}{\psi_{3,1}}\left(z+z_{o}\right)^{2}-\frac{C_{2} h_{c} U_{o} d_{o} \psi_{0,1,2}}{A \psi_{3,1}}=0
\end{gathered}
$$

for the two models, respectively. Solving for $\beta_{c}$ in Eq. (4.73b) then gives

$$
\beta_{c}=\sqrt{\left(\frac{\tau_{o}}{\rho_{\infty}} \frac{1}{A^{2}} \frac{\psi_{1,0}}{\psi_{3,1}}\left(z+z_{o}\right)^{2}\right)^{2}+2 \frac{C_{2} h_{c} U_{o} d_{o} \psi_{0,1,2}}{A \psi_{3,1}}}-\frac{\tau_{o}}{\rho_{\infty}} \frac{1}{A^{2}} \frac{\psi_{1,0}}{\psi_{3,1}}\left(z+z_{o}\right)^{2}
$$

Because Eq. (4.73a) is a cubic equation, it may be solved similarly, but no single line solutions exist.

There are two essential messages from this result. First, the ersatz constants $A$ and $\beta_{c}$ are not really constants but depend on elevation. Therefore, the radial distribution is not a constant like the free jet but may depend on elevation. Second, unlike the free jet, the energy and momentum equations cannot be artificially separated. Indeed, although solution of the coupled equations is necessary to fully understand how the jet flows, Eq. (4.74) suggests that the jet starts out at the base with a width that is similar to the free jet. As the jet rises in elevation, the yield stress terms show that the radial spread would eventually vanish (in the absence of a turbulent-to-laminar transition). Figure 6 in Shekarriz et al. (1995a) hints that $\delta_{c}$ decreases with elevation as implied by Eq. (4.74). Therefore, in the absence of solving the coupled equations, Section 6 examines three limited cases: a dispersive turbulent expanding jet as would be anticipated near the base of the upwell, a jet of constant diameter (this may exist at some point along the 
jet trajectory if it starts divergent but mathematically ends with zero width), and a laminar upwell of constant diameter. These limiting cases in Section 6 should be appreciated for what they are: insightful approximations that guide consideration of scaling between vessels that are supported by experimentally obtained data to the extent available.

\subsection{Summary}

This section establishes the governing differential equations for momentum, energy and species conservation (see Eq. 4.18, 4.26, and 4.40). Both buoyancy and yield stresses are included providing a general framework for axisymmetric jets in a variety of fluids. The Froude number based on nozzle diameter without densities (Eq. 4.22) is the appropriate Froude number for jet flows (see Section 5). The density profile as a function of elevation and radial position is estimated in the absence of buoyancy forces (Eq. 4.51 and 4.53). The turbulence length scale governs the jet width (Eq. 4.61) and settling velocities are shown to be negligibly small compared to other velocities within the center of the jet. See Sections 5 and 6 for the implications of these developments for cloud and "cavern" heights. 



\subsection{Gravitational Forces}

This section begins consideration of mechanisms or flow regimes that confine or limit the free jet described in Section 3. Section 4 developed the governing equations for a jet influenced by viscous stresses, buoyancy forces, yield stress, and jet transience. Viscous forces were shown to be negligible relative to turbulence and will not be considered further. Where turbulence is suppressed by another mechanism, viscous forces may become important again, but the essential focus here is on the first mechanism to impede the free jet. This section then considers the influence of buoyancy forces in terms of the Froude number, and the cloud height model described in Section 4 is benchmarked against experimental data. Implications for surface flow and dependence on geometric scaling are discussed.

\subsection{Negative Buoyancy, the Froude Number, and Fountains}

Section 4 showed that buoyancy forces may be scaled on the Froude number. The governing momentum equation clarified which of the various forms of the Froude number is relevant to the hydrodynamic jet physics that govern the upwell. Eq. (4.22) (repeated below) showed that the only Froude number that included scale factors that were not a function of position and satisfied conservation of momentum for the central upwell itself was

$$
\mathrm{Fr}=\frac{U_{o}{ }^{2}}{g d_{o}}
$$

Although the Froude number clearly plays an integral role in negative buoyancy arguments, the precise value at which the Froude number may predict failure of the central upwell remains unclear. This Froude number is termed the critical Froude number, $\mathrm{Fr}_{\mathrm{c}}$.

The critical Froude number may be determined from experimental data compiled from $8 \mathrm{ft}$ vessel tests. This dimensionless number is plotted in Figure 5.1 against the surface flow criterion for the tests described in Table 2.3. The figure shows that surface flow is achieved at higher Froude numbers, and at lower Froude numbers the upwell fails to reach the surface. The figure shows that for tests with a nozzle diameter of 1.94 inches, there is a reasonable critical Froude number discerning between tests for which surface flow was reported and those for which it was not of approximately 285. Tests for which surface flow observation was "at or near the surface" (designation by $1 / 2$ on the $x$-axis) span the critical Froude number. Remarkably, all of the firing sequences (S, A1, A2), pulse or drive volume fractions, and both the two-part simulant of run sheet 1 and the multipart simulant of run sheet 7 are agreed on the same cutoff between those that definitely induce surface flow and those that do not. That all the simulants for a particular nozzle size are distinguished by one critical Froude number indicates that further exploration of densities remains unnecessary and even unhelpful. 


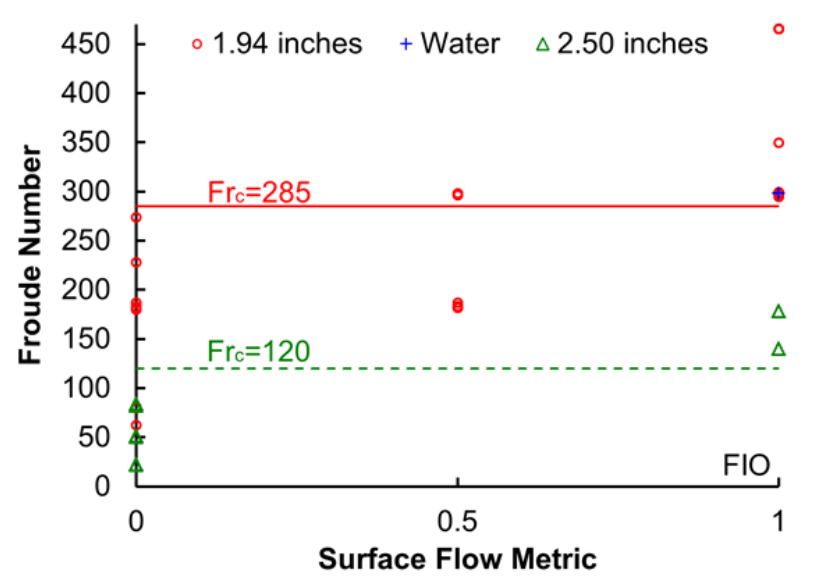

Figure 5.1. The Froude Number Differentiated on the Surface Flow Metric of Table 2.3

However, the tests that used a 2.50 inch nozzle diameter appear to be of a different population. The cutoff for this set is approximately $\mathrm{Fr}_{\mathrm{c}}=120$. That there would be a different cutoff between the two nozzle diameters is surprising. Typically, one would argue that the characteristic length, here the nozzle diameter, is the key to scaling between vessel sizes. With geometric scaling, the nozzle diameter doubles, so to achieve the same critical Froude number the velocity would have to increase by the square root of 2 . Yet, it appears that the nozzle diameter also affects the value of the critical Froude number, complicating this argument. Indeed, a decreasing critical Froude number with increasing scale would suggest it may be even easier to overcome negative buoyancy in scaled-up vessels. However, with only two nozzle sizes explored in one vessel size, it is not feasible to determine a reliable critical Froude number for scale-up from this data. Furthermore, attempts to use a vessel diameter instead of a nozzle diameter clearly would not cause these curves to collapse, suggesting that the disagreement does not boil down to an argument over the characteristic length scale. Neither a Froude number based on nozzle diameter nor a Froude number based on vessel diameter collapses the data sufficiently to enable scaling between vessel sizes. Nevertheless, based on these results, one would anticipate that a single critical Froude number for a variety of simulants and firing sequences would emerge in tests in the $16 \mathrm{ft}$ vessel.

The values of the Froude number may seem surprisingly high; however, this is not unreasonable for four reasons. First, intuition around the Froude number has been built on the fountain height literature that focuses on Froude numbers in the range of 1 to 20 (Srinarayana et al. 2008, 2009a, b). However, fountain height arguments and Froude number arguments are typically reserved for cases where distinctive free surfaces are present with correspondingly large changes in density across the interface. No distinctive free surface or density gradients that span orders of magnitude are present in the central upwell. In contrast, they are present at the fluid surface, and one may anticipate that traditional fountain height arguments would be validated up there (see Section 6.3). Second, fountain height arguments often use the square root of the Froude numbers in this document (Srinarayana et al. 2008, 2009a, b). This would lower the critical Froude numbers to 11.0 and 16.9 for $d_{o}=2.50$ and 1.94 inches, respectively. These are of the same order of magnitude as those studied in the fountain height literature, with larger numbers leading to stronger, more turbulent fountains. Third, although preferable to have a critical value of a dimensionless group around unity, not all dimensionless numbers necessarily indicate a change in flow regimes around unity. Classic examples include the persistence of laminar flow pipe until a Reynolds number of $\sim 1800$ (converging flows have remained laminar out to $>5 \cdot 10^{5}$ ) and critical Rayleigh numbers 657 to 1708 for the Benard problem involving the stability of fluid heated from below (Deen 
2012). Fourth, negative buoyancy is proportional to the inverse of the Froude number, so a large Froude number would suggest that the rate of momentum addition is much stronger than negative buoyancy forces. However, the jet momentum also decays as the jet spreads due to entrainment of peripheral fluid. Indeed, the rate of momentum addition decays with the scaled elevation. Where these two balance, a cloud height appears, but making the Froude number spatially dependent is unsatisfying and makes scaling arguments intricate if not unwieldy.

Please note that when only two PJMs operate, the momentum spreads out perpendicular to the cross section of the jet such that there is much less flow. This would substantially affect the Froude number analysis for those configurations. These results should not be extrapolated to that flow configuration.

\subsection{Cloud Height}

The use of cloud height correlations to predict surface flow is supported by the observation that when the cloud height reaches the upper liquid surface, surface flow must have been achieved. Table 2.3 shows that the cloud height is closely correlated with surface flow. ${ }^{1}$ Cloud heights for various PJM arrays (typically distinctive from the one in the proposed standard high solids vessel) were correlated in Meyer et al. (2012) as discussed by Wells et al. (2015).

Rigorous determination of the cloud height from first principles requires simultaneous solution of Eqs. (4.18) or (4.26) and (4.40) for momentum and species conservation, respectively, in conjunction with the corresponding energy balance (see Section 4.3). The cloud height may be approximated, however, by asserting knowledge of the density profiles derived in Section 4.2 and then back-solving for the velocity profiles in the spirit of a successive substitution method. Simplifying Eq. (4.18) (Eq. (4.26) leads to the same result) for steady state, small-scaled pressure gradients in the absence of yield stresses at modest to high jet Reynolds numbers leads to

$$
\int_{0}^{\infty} \bar{v} \frac{\partial \bar{u}}{\partial \bar{r}} \bar{r} d \bar{r}+\int_{0}^{\infty} \bar{u} \frac{\partial \bar{u}}{\partial \bar{z}} \bar{r} d \bar{r}=-\frac{1}{F r} \int_{0}^{\infty} \bar{\rho}(\bar{\rho}-1) \bar{r} d \bar{r}+\int_{0}^{\infty} \frac{C^{2}}{\beta_{c}^{3}} \frac{1}{\bar{r}} \frac{\partial}{\partial \bar{r}}\left[\bar{r}\left(\bar{z}+\bar{z}_{o}\right)^{2}\left(\frac{\partial \bar{u}}{\partial \bar{r}}\right)^{2}\right] \bar{r} d \bar{r}
$$

The final term vanishes for any of the profiles in Table 3.1, and the first terms may be combined via integration by parts and continuity (Rajaratnam 1976) to give

$$
\frac{d}{d \bar{z}} \int_{0}^{\infty} \bar{u}^{2} \bar{r} d \bar{r}=-\frac{1}{F r} \int_{0}^{\infty} \bar{\rho}(\bar{\rho}-1) \bar{r} d \bar{r}
$$

\footnotetext{
${ }^{1}$ The cloud height in these tests was measured at the wall, not in the center of the vessel, and the Meyer et al. (2012) correlations were prepared for cloud height observations at the vessel center. The two can be expected to be the same only if the cloud height is driven by the central upwell and the primary direction of flow at the cloud height is from center to wall in a time-averaged sense. If the primary direction of flow is upward along the wall so that the cloud height is determined by this flow (not the central upwell), then one cannot expect the Meyer et al. (2012) correlations to apply. Section 8 suggests that the flows along the wall remain too weak to serve as the primary driver of the cloud height in the first instance.
} 
Eqs. (4.51) and (4.53) with densities may be scaled on $\rho_{\infty}$ to give

$$
\bar{\rho}=\frac{\bar{\rho}_{c}}{\left(1-\bar{\rho}_{c}\right) f_{c}^{S c_{t}}+\bar{\rho}_{c}}
$$

and

$$
\bar{\rho}_{c}=\frac{\bar{\rho}_{\max }}{\frac{\bar{z}_{o}}{\bar{z}+\bar{z}_{o}}\left(1-\bar{\rho}_{\max }\right)+\bar{\rho}_{\max }}
$$

Bringing derivatives and constants into the integral of Eq. (5.2) admits

$$
\frac{\partial}{\partial \bar{z}} \bar{u}^{2}=-\frac{1}{F r} \bar{\rho}(\bar{\rho}-1)
$$

at any point, particularly along the vessel centerline. This leads to the most optimistic definition of a cloud height in keeping with the experiments (Bontha et al. 2105). Integration of only the centerline components (forcing $f_{c}=1$; a first order approximation indeed but perhaps no more egregious than working in the dilute species approximation for water, assuming a free jet density profile in the presence of solids, or decoupling energy from the momentum balance and still more insightful than regressive correlation) then gives

$$
\bar{u}_{m, c}{ }^{2}=-\int_{0}^{\bar{z}} \frac{1}{F r} \bar{\rho}_{c}\left(\bar{\rho}_{c}-1\right) d \bar{z}+\frac{N f_{N}}{8 \psi_{2,1}} \frac{\rho_{o}}{\rho_{\infty}} \frac{1}{\beta_{c}^{2}\left(\bar{z}+\bar{z}_{o}\right)^{2}}
$$

where the integration "constant" was suggested by Eq. (3.8). Substituting Eq. (5.4) gives

$$
\bar{u}_{m, c}{ }^{2}=\frac{\bar{z}_{o} \bar{\rho}_{\max }\left(1-\bar{\rho}_{\max }\right)}{F r} \int_{0}^{\bar{z}} \frac{\left(\bar{z}+\bar{z}_{o}\right)}{\left[\bar{z}_{o}+\bar{z} \bar{\rho}_{\max }\right]^{2}} d \bar{z}+\frac{N f_{N}}{8 \psi_{2,1}} \frac{\rho_{o}}{\rho_{\infty}} \frac{1}{\beta_{c}{ }^{2}\left(\bar{z}+\bar{z}_{o}\right)^{2}}
$$

The integral may then be resolved directly as

$$
\bar{u}_{m, c}{ }^{2}=-\frac{\bar{z}_{o}\left(\bar{\rho}_{\max }-1\right)}{F r \bar{\rho}_{\max }}\left[\ln \frac{\bar{z}_{o}+\bar{z} \bar{\rho}_{\max }}{\bar{z}_{o}}+\frac{\bar{z} \bar{\rho}_{\max }\left(\bar{\rho}_{\max }-1\right)}{\bar{z}_{o}+\bar{z} \bar{\rho}_{\max }}\right]+\frac{N f_{N}}{8 \psi_{2,1}} \frac{\rho_{o}}{\rho_{\infty}} \frac{1}{\beta_{c}{ }^{2}\left(\bar{z}+\bar{z}_{o}\right)^{2}}
$$

It may be shown for small relative densities (e.g., $\rho$ ranges from 0.995 to $1.058 \mathrm{~g} / \mathrm{mL}$ for run sheets 1 and 2) that the second term in the brackets is small relative to the first, leaving

$$
\bar{u}_{m, c}{ }^{2}=-\frac{\bar{z}_{o}\left(\bar{\rho}_{\max }-1\right)}{F r \bar{\rho}_{\max }} \ln \frac{\bar{z}_{o}+\bar{z} \bar{\rho}_{\max }}{\bar{z}_{o}}+\frac{N f_{N}}{8 \psi_{2,1}} \frac{\rho_{o}}{\rho_{\infty}} \frac{1}{\beta_{c}^{2}\left(\bar{z}+\bar{z}_{o}\right)^{2}}
$$

from which one may argue for a density-dependent Froude number that will be one to three orders of magnitude larger than those considered above. 
The final term may be rewritten in terms of $h_{c}$ as defined previously as

$$
\bar{u}_{m, c}{ }^{2}=-\frac{\bar{z}_{o}\left(\bar{\rho}_{\max }-1\right)}{F r \bar{\rho}_{\max }} \ln \frac{\bar{z}_{o}+\bar{z} \bar{\rho}_{\max }}{\bar{z}_{o}}+\frac{h_{c}{ }^{2}}{\left(\bar{z}+\bar{z}_{o}\right)^{2}}
$$

This expression shows that the negative buoyancy of the solids opposes the upward momentum of the central upwell. Setting the velocity to zero identifies $z$ as a cloud height, $z_{\text {cloud }}$. However, the expression remains transcendental such that a simple analytical expression does not naturally emerge, but the expression is sufficiently straightforward to be evaluated directly. Remarkably, this expression argues that the cloud height depends explicitly on only four parameters: a Froude number, a density ratio, a jet coefficient, and a virtual origin. Because the virtual origin has not been considered in extensive detail previously, it may contribute uncertainty to prior analyses of cloud height. Figure 3.1 provides a relationship between $z_{o} / d_{o}$ and $h_{c}$ that may be useful (but incompletely benchmarked) in further establishing the virtual origin in this equation.

It is important to note that the cloud height arguments herein assert that particles that make it into the upwell will all move together in the core of the jet as long as the settling velocities are sufficiently small (the case for the six-part simulant). This analysis in no way predicts that the cloud height is independent of solids loading or slurry density - quite the opposite is true.

Figure 5.2 compares the cloud heights predicted by Eq. (5.10) to experiments using the two-part simulant of run sheets 1 and 2 . The values of $h_{c}$ and $z_{o} / d_{o}$ for the two-part simulant are given in Table 2.4 and only the average values $h_{c}$ and $z_{o} / d_{o}$ for which surface flow was achieved were used regardless of whether an individual test achieved surface flow or not. The Froude numbers were calculated based on the nozzle velocity and diameter for each test in Table 2.3 from run sheets 1 and 2, and the density ratio was estimated by taking the maximum and minimum densities tabulated in Appendix G of Bontha et al. (2015), again specific to each test. Figure 5.2a shows reasonable agreement between prediction and experiment, except where the model underpredicts the cloud height (a conservatism), provided that the vessel was filled to at least the predicted cloud height. Figure 5.2b highlights the model's ability to approximately distinguish between surface flow and no surface flow. Notably, the model does not include the finite thickness of the cloud itself, which may be a few nozzle diameters thick.

Figure 5.2c uses the model to anticipate the cloud height for various cases. This cloud height model depends on only four parameters: $h_{c}, \mathrm{Fr}, \rho_{\max } / \rho_{\infty}$, and $z_{o} / d_{o}$. Of these four, only $h_{c}$ may be argued to be scale independent or does not change with geometric scaling (see Section 3). Of the four, Fr clearly depends on geometric scale because it is inversely proportional to nozzle diameter (the diameter dependence of $\mathrm{Fr}_{\mathrm{c}}$ does not complicate the arguments here). The dependence of $\rho_{\max } / \rho_{\infty}$ and $z_{o} / d_{o}$ on geometric scaling has not been established in this analysis. Such analyses are feasible, straightforward, and likely quite informative. In the meantime, to explore the influence of these parameters on the cloud height, Figure 5.2c considers what may happen in the $16 \mathrm{ft}$ vessel if $h_{c}$ and $z_{o} / d_{o}$ do not change between the 8 and $16 \mathrm{ft}$ vessels (an unproven assumption) holding $U_{o}$ constant at $12 \mathrm{~m} / \mathrm{s}$. The figure shows that for values of the two-part simulant or the six-part simulant, approximately doubling the nozzle diameter decreases the anticipated cloud height by $\sim 10$ to 20 nozzle diameters or $\sim 40$ to 80 inches in the $16 \mathrm{ft}$ vessel. Going from a two-part simulant to a six-part simulant modestly changes $h_{c}$ from 4.26 to 4.35 and $z_{o} / d_{o}$ from 32.8 to 28.7 . This change also adjusts the cloud height by an additional $\sim 5$ nozzle diameters. 
Additional changes due to the density ratio may increase or decrease the cloud height between vessel sizes.

Figure $5.2 \mathrm{~d}$ shows the measured maximum and minimum slurry densities along with the density ratio for run sheets 1 and 2. This figure suggests that the preferred means of improving the predictive capability of the cloud height model is to model the densities that arise using species conservation associated with impinging radial wall jets along the vessel bottom because the density ratio most closely matches the maximum density. A similar analysis was completed by Rao (1980), which with thoughtful revision may be serviceable for this purpose with boundary conditions proposed by Kuhn et al. (2013, Sections 2.2.2.2, A.6, B.8.1) or Wells et al. (2009) in conjunction with revisions of the Prandtl mixing length for slurries by Masuyama et al. (1980). The density far away from the centerline contributes less than $\sim 1 / 4$ of the variation and may be analyzed based on the settling analysis in Chapter 12 of Russel et al. (1989) with Bingham number corrections to Stokes law (Kuhn et al. 2013, Section E.6.2.1.1.2) as appropriate. ${ }^{1}$ Figure 5.2d does not suggest that the slurry densities are constant at higher nozzle velocities because the figure of merit in Eq. (5.10) is $\rho_{\max } / \rho_{\infty}-1$. The density ratio varies from slightly under 1.02 to above 1.06. Subtracting one shows that this modest density variation is approximately a factor of three in variation. Therefore, precise modeling of this value is important to extending this cloud height model from a descriptive model of historical experiments into a predictive tool for scale up. Figure 5.2d does not imply or suggest what the density ratio might or might not be for other simulants or operating conditions.

\footnotetext{
${ }^{1}$ Interparticle forces should not be neglected in such analyses because the settling rate depends strongly on these forces, which may accelerate (even without aggregation) or attenuate the settling velocity relative to the hard sphere approximation usually entertained in analyses for the WTP; concentration effects alone are insufficient to produce predictive models sufficiently accurate for the present purposes (Kuhn et al. 2013, Section C.4.2.10).
} 


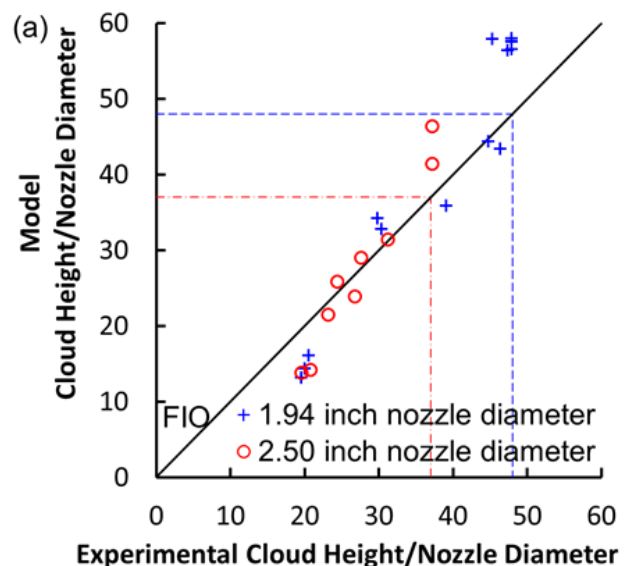

Experimental Cloud Height/Nozzle Diameter

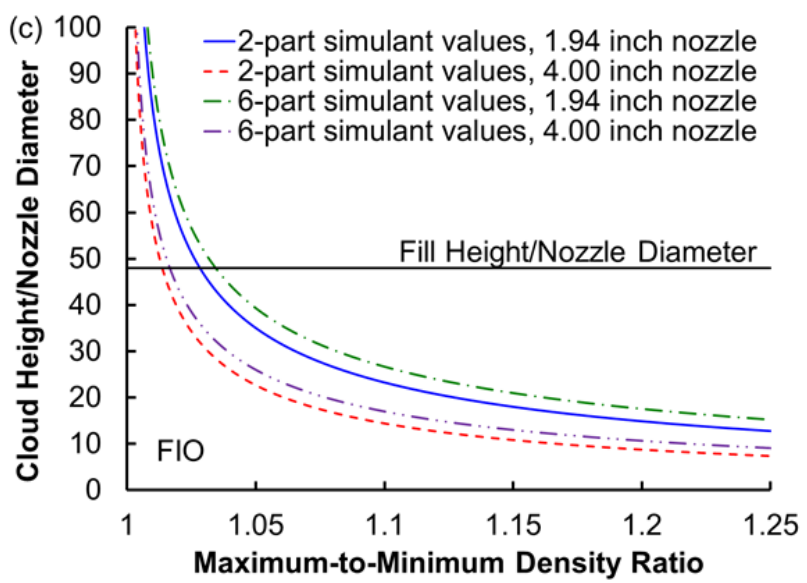

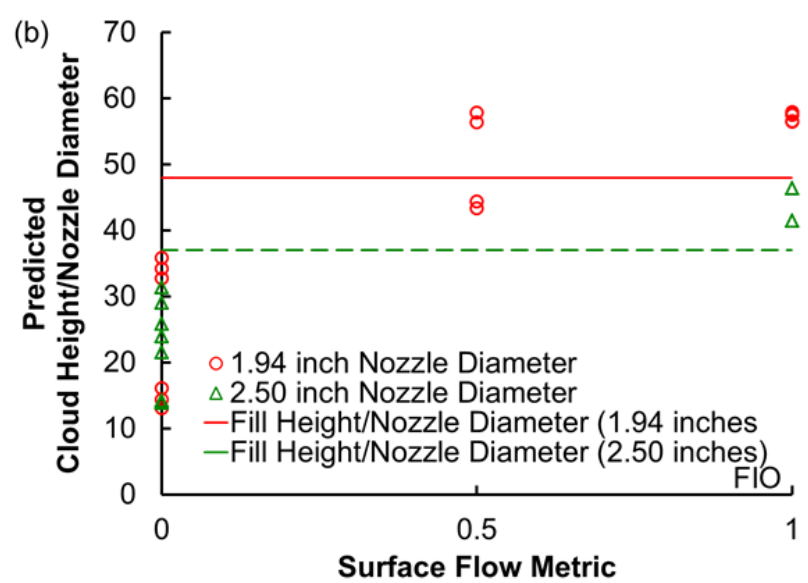

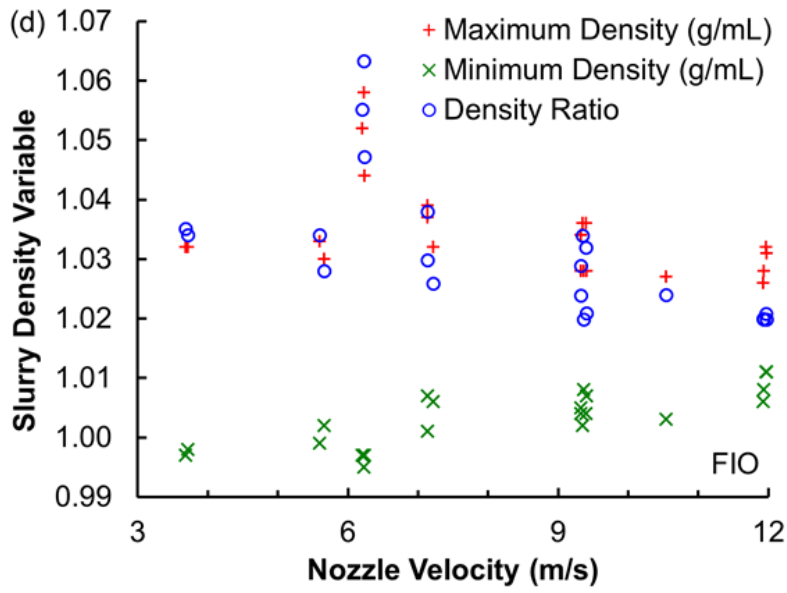

Figure 5.2. (a) Model Prediction versus Experimentally Obtained Values of the Cloud Height Scaled on Nozzle Diameter for the Two-Part Simulant of Run Sheets 1 and 2 (Table 2.3) Based on Densities from Bontha et al. (2015, Appendix G) and $h_{c}$ and $z_{o} / d_{o}$ at Surface Flow Conditions per Table 2.4 Compared to Fill Height/Nozzle Diameter for $d_{o}=1.94$ inches (dash, blue) and 2.50 inches (dash dot, red). (b) Model Cloud Height Scaled on Nozzle Diameter Differentiated on Surface Flow Metric of Table 2.3. (c) Model Cloud Height Scaled on Nozzle Diameter versus $\rho_{\max } / \rho_{\infty}$ for the Two-Part Simulant with a 1.94 inch Nozzle Diameter (blue solid: $\left.\mathrm{Fr}=298, h_{c}=4.26, z_{o} / d_{o}=32.8\right)$ and Extrapolated for the Two-Part Simulant with a 4 inch Nozzle Diameter (red dash: $\mathrm{Fr}=144, h_{c}=4.26, z_{o} / d_{o}=32.8$ ), the Six-Part Simulant with a 1.94 inch Nozzle Velocity (green dot dash: $\mathrm{Fr}=298, h_{c}=4.35, z_{o} / d_{o}=28.7$ ), and the Extrapolated for the Six-Part Simulant with a 4 inch Nozzle Velocity (purple double dot dash: $\mathrm{Fr}=144, h_{c}=4.35, z_{o} / d_{o}=28.7$ ). (d) Measured Minimum and Maximum Slurry Densities from Appendix G of Bontha et al. (2015) versus Nozzle Velocity with the Density Ratio Thereof.

Equation (5.10) not only predicts the cloud height but also predicts the precipitous decay in centerline velocity near the cloud height. For example, Figure 5.3 presents the centerline velocity as a function of elevation in the $12.75 \mathrm{ft}$ vessel. Panel a shows the centerline velocity using Eq. (5.10), and panel $\mathrm{b}$ shows the centerline velocity for the free jet using Eq. 2.1. Remarkably, Equation (5.10) succinctly captures the 
more rapid decay in centerline velocity that the free jet curve simply missed. However, when the Fr is very large or the density gradients vanish, Eqs. (5.10) and (2.1) converge. Finally, Bontha et al. (2003b) observed that all four tests homogeneously mixed the vessel contents. This observation is approximately consistent with the observed velocity profiles because the cloud height for the $20 \% 35$ micron glass beads lies at or near the top of the fluid in this vessel (see Table 5.1).
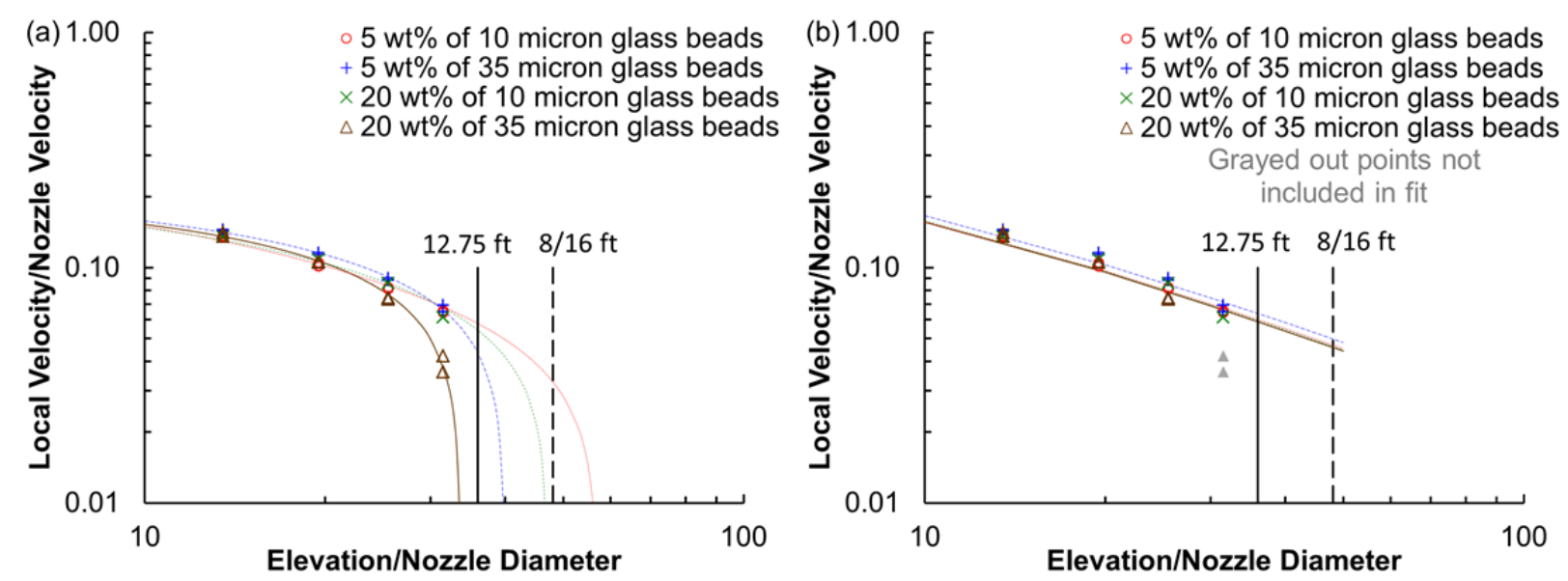

Figure 5.3. Centerline Velocity Scaled on Nozzle Velocity Versus Elevation Scaled on a Nozzle Diameter Comparing (a) Eq. (5.10) and (b) Eq. (2.1). See Table 5.1 for parameters used to fit panel a. Vertical lines at $z / d_{o}=36$ and 48 represent the dimensionless fill height of the $12.75 \mathrm{ft}$ vessel $\left(z / d_{o}=29-36\right.$ in Bontha et al. $\left.2003 \mathrm{~b}\right)$ and 8 or $16 \mathrm{ft}$ standard high solids vessel designs, respectively.

Table 5.1 shows the values of the parameters that were used to obtain the curves in Figure 5.3a. The Froude number was determined directly from Eq. (4.22) and does not vary among the tests. The jet coefficient, $h_{c}$, was not fit to the data but was quantified using Eq. (3.9). The remaining parameters, $z_{o} / d_{o}$ and $\rho_{\max } / \rho_{\infty}$ were fit by minimizing the square of the difference between predicted and experimental velocities, by rastering across anticipated parameter space, and by ensuring that the resulting values lie within (not on the edge of) their respective domains. These parameters are remarkable for three reasons. First, the value of $h_{c}$ was determined not by fitting but directly from conservation of mass indicating the utility of the predictions in Section 3. The doubling in $h_{c}$ falls directly from the decreases in $\beta_{c}$ from the radial distributions of Tables 2.1 and 3.1, showing that the jet spread significantly affects the value of the jet coefficient and subsequent velocity predictions. Second, the parameters representing the virtual origin and the density ratio increase with increasing weight fraction. Third, the $\left(h_{c}, z_{o} / d_{o}\right)$ ordered pairs lie within the uncertainty of those given in Figure 3.1, supporting the approximately linear correlation between the two parameters. 
Table 5.1. The Values Used to Fit Eq. (5.10) in Figure 5.3a

\begin{tabular}{llcccc}
\hline Reference & \multicolumn{1}{c}{ Fluid } & $h_{c}$ & $z_{o} / d_{o}$ & $\rho_{\max } / \rho_{\infty}$ & $z_{\text {cloud }} / d_{o}$ \\
\hline Bontha et al. (2003b) & $5 \% 10 \mu$ m glass beads & 3.73 & 14.4 & 1.009 & 57.5 \\
Bontha et al. (2003b) & $20 \% 10 \mu$ m glass beads & 5.02 & 22.7 & 1.016 & 47.2 \\
Bontha et al. (2003b) & $5 \% 35 \mu$ m glass beads & 7.63 & 35.3 & 1.030 & 39.9 \\
Bontha et al. (2003b) & $20 \% 35 \mu$ m glass beads & 8.39 & 40.0 & 1.041 & 33.6 \\
\hline
\end{tabular}

Determined by finding the minimum with Fr=77.0 from Eq. $4.22, h_{c}$ from Eq. 3.9 with $c_{j}=1$ and $\rho_{o} / \rho=1$, and $z_{o} / d_{o}$ and $\rho_{\max } / \rho_{\infty}$ as least squares fitting parameters in the domains of [10.0-80.0] and [1.0001-1.1001], respectively.

\subsection{Summary}

This section evaluates the influence of buoyancy forces in solids laden jets. Though the Froude number defined on nozzle diameter collapses the data onto one plot (Figure 5.1), the critical Froude number to determine whether the upwell jet does or does not reach the fluid surface depends on nozzle diameter even when the vessel diameter is held constant. Because this criteria to date has only been evaluated for one vessel size and two nozzle diameters, further dependencies on vessel diameter remain unclear. Therefore, the Froude number and critical Froude numbers are insufficient bases to reliably predict performance of full scale jets from smaller scale data without further determination of the critical Froude number at other vessel sizes and nozzle diameters.

Alternatively, a new four parameter cloud height model (Eq. 5.10) was developed and benchmarked against data at two scales: $8 \mathrm{ft}$ and $12.75 \mathrm{ft}$. This model not only determined the cloud height where input parameters are known but is also consistent with the decay in axial velocities as a function of elevation using jet coefficients predicted in Section 3 for a free jet. This model falls short of predicting performance at larger scales because model development to predict a density ratio and depth of the virtual origin lie outside the scope of this report. 



\subsection{Yield Stresses}

This section considers the influence of yield stresses on jet flows and evaluates the implications of the confinement these stresses may induce. Expanding jets and constant diameter turbulent and laminar flows are considered. Critical elevations at which the flows terminate are benchmarked against "cavern" height data, though the expressions developed herein are for a conical or tubular geometry instead of a broad cavern (characteristic of impeller flow). Implications for breakthrough and dependence on geometric scaling are discussed.

\subsection{Turbulent Expanding Jets in Yield Stress Materials}

Section 4 developed a mathematical framework to evaluate momentum transfer in the presence of hypothesized yield stresses (see final footnote in Section 2.2). These yield stresses would attenuate the momentum as developed herein and at least partially alter the velocity profile (see Section 2.2). Jets in non-Newtonian materials have been considered by a variety of authors, including Shekarriz et al. (1995a, 1995 b, 1997), Powell et al. (1997), and Cai et al. (2012). This section considers turbulent jets influenced by a yield stress but that continue to expand, albeit weakly as observed in Figure 2.4. Yield stresses that further confine the jet to a constant diameter tube are considered in subsequent sections.

Section 4 considers conservation of momentum. Assuming the existence of a physical yield stress and at steady state in the absence of significant body forces ${ }^{1}$ and negligible viscous stresses relative to the turbulent Reynolds stresses, both turbulence models reduce to Eq. (4.70). Integrating over $z$ then gives

$$
\int_{0}^{R_{j e t}(z)} 2 \pi \rho_{\infty} u^{2} r d r=-2 \pi \tau_{w} \int_{0}^{z} R_{j e t}(z) d z+M_{o}
$$

where $\tau_{o}=-\tau_{w}$ (appropriate when the viscous stresses based on the Bingham consistency parameter remain small as expected of a turbulent jet). ${ }^{2}$ One may immediately recognize the left-hand side as the momentum at any point along the jet. The factor of $2 \pi$ gives the traditional form of this expression (Powell et al. 1997; Rajaratnam 1976). The first term on the right represents the momentum lost to shear stresses and the second term and integration constant is the entering momentum. It is important to note here that this derivation only holds for true yield stress materials. This development does not hold for other rheologies that have only an apparent yield stress, including generalized Newtonian, power law, and colloidal rheologies (Larson 1999; Shekarriz et al. 1997). Apparent yield stresses from extrapolating power law fluids to low stresses, for example, do not satisfy this requirement. The earlier development herein without a yield stress applies for each of these rheologies without modification as long as the viscous stresses remain much smaller than turbulent stresses. The form of $R_{\text {jet }}$ may be determined by rearranging Eq. (2.4) as

$$
R_{j e t}(z)=\eta_{c}^{99} \beta_{c}\left(z+z_{o}\right)
$$

\footnotetext{
${ }^{1}$ It is possible to have a combination of small, repulsive particles (see final footnote in Section 2.2) and large, dense particles where both yield stresses and buoyancy together would be important. This case is not considered herein, but this framework enables evaluation of this adverse case.

${ }^{2}$ Rajaratnam (1976) corrects a minor sign error in Powell et al. (1997) on the right-hand side of this equation.
} 
where $\eta_{c}{ }^{99}$ represents the scaled position that encompasses $99 \%$ of the jet flow defined by $f_{c}\left(\eta_{c}{ }^{99}\right)=0.01$ as tabulated in Table 3.1. This approach is similar to but more general than that of Powell et al. (1997), who assert $R_{j e t}(z)=0.2 z$ by similar arguments. Substituting and evaluating the first term as in Section 3 gives

$$
2 \pi \rho_{\infty} u_{m, c}{ }^{2} \beta_{c}^{2}\left(z+z_{o}\right)^{2} \psi_{2,1}=-2 \pi \tau_{w} \eta_{c}^{99} \beta_{c}\left(\frac{1}{2} z^{2}+z_{o} z\right)+M_{o}
$$

The value of $\beta_{c}$ is given in Table 2.1 and $\psi_{2,1}$ in Table 3.1. Solving for the velocity with Eq. (3.1) finds

$$
u_{m, c}=\frac{c_{j}}{\beta_{c}} \sqrt{\frac{N f_{N}}{8 \psi_{2,1}} \frac{\rho_{o}}{\rho_{\infty}}-\frac{\rho_{o}}{\rho_{\infty}} \frac{\tau_{w}}{\rho_{o} U_{o}{ }^{2}} \frac{\eta_{c}^{99} \beta_{c}}{\psi_{2,1}} \frac{\left(\frac{1}{2} z^{2}+z_{o} z\right)}{d_{o}{ }^{2}}} \frac{U_{o} d_{o}}{z+z_{o}}
$$

which shows that the yield stress term is a function of position where $z_{o}$ is nonzero, where $c_{j}$ is an additional fitting constant. In scaled form,

$$
\frac{u_{m, c}}{U_{o}}=\frac{c_{j}}{\beta_{c}} \sqrt{\frac{N f_{N}}{8 \psi_{2,1}} \frac{\rho_{o}}{\rho_{\infty}}-\frac{\rho_{o}}{\rho_{\infty}} \frac{1}{\operatorname{Re}_{\tau}} \frac{\tau_{w}}{\tau_{o}} \frac{\eta_{c}^{99} \beta_{c}}{\psi_{2,1}}\left[\frac{1}{2}\left(\frac{z}{d_{o}}\right)^{2}+\frac{z_{o}}{d_{o}} \frac{z}{d_{o}}\right]} \frac{1}{z / d_{o}+z_{o} / d_{o}}
$$

The densities have been retained throughout, but with the KB slurry, the change in slurry density is likely modest (an unproven assumption not evaluated herein). The elevation where the momentum exhausts may be determined by setting the left-hand side of this equation to zero as the velocity vanishes. Then

$$
\frac{1}{2}\left(\frac{z_{c}}{d_{o}}\right)^{2}+\frac{z_{o}}{d_{o}} \frac{z_{c}}{d_{o}}-\frac{N f_{N}}{8 \eta_{c}^{99} \beta_{c}} \frac{\tau_{o}}{\tau_{w}} \operatorname{Re}_{\tau}=0
$$

where $z_{c}$ is the critical elevation ${ }^{1}$ where the momentum exhausts, assuming the self-similar profile initiated with the expanding jet persists. Solving the quadratic equation in $z_{c}$ finds

$$
\frac{z_{c}}{d_{o}}=\sqrt{\left(\frac{z_{o}}{d_{o}}\right)^{2}+\frac{N f_{N}}{4 \eta_{c}{ }^{99} \beta_{c}} \frac{\tau_{o}}{\tau_{w}} \operatorname{Re}_{\tau}}-\frac{z_{o}}{d_{o}}
$$

for $z_{o} / d_{o} \geq 0$. Here the critical elevation depends approximately on the square root of the yield Reynolds number, though the presence of a virtual origin makes the dependence modestly more intricate. In the hypothetical absence of a virtual origin,

$$
\frac{z_{c}}{d_{o}}=\sqrt{\frac{N f_{N}}{4 \eta_{c}^{99} \beta_{c}} \frac{\tau_{o}}{\tau_{w}} \operatorname{Re}_{\tau}}
$$

\footnotetext{
${ }^{1}$ The term "critical elevation" suggests a linear measurement along the vessel centerline instead of "cavern" height because the former suggests a linear measurement of jet influence instead of a volumetric measurement suggested by "cavern" height. This difference lacks distinction in some cases but is essential in others.
} 
The approximate square root dependence is qualitatively similar to the "cavern" height arguments (Bamberger et al. 2005), although the geometric arguments remain markedly different. "Caverns" suggest a bulk volume whereas this expression arises from only a conical central upwell. However, Section 2 showed virtual origins to be non-negligible in tertiary flows.

Prior to achieving the surface, the leading edge of the jet also sustains shear stresses. The exact time-averaged shape of the leading vortex in slurries remains unclear. The experiments of Cai et al. (2012), who experimentally evaluated a sand jet in laponite, suggest that the leading edge of a confined jet neither vanishes nor remains self-similar but takes on a hemispherical morphology. Cai et al. (2012) give the shear force (a rate of momentum) on a hemispherical head in the notation as

$$
F_{\text {shear }}=\frac{\pi^{2}}{2} R_{\text {jet }}^{2} f_{\text {vortex }} \tau_{w}
$$

where the diameter of the head is equal to the diameter of the jet below multiplied by a factor of $f_{\text {vortex }} \geq 1$ to represent the lateral extent of the leading vortex, which may be greater than the jet radius. (The radial extent of the leading vortex in this formulation is $R_{\text {jet }} f_{\text {vortex }}^{1 / 2}$.) The diameter of leading vortices exceeds that of the trailing jet. Additional shear stresses from flow in the head are assumed to be negligible.

Then, an integral force balance gives

$$
\int_{0}^{R_{j e t}(z)} 2 \pi \rho_{\infty} u^{2} r d r=-2 \pi \tau_{w} \int_{0}^{z} R_{j e t}(z) d z-\frac{\pi^{2}}{2} R_{j e t}^{2} f_{v o r t e x} \tau_{w}+M_{o}
$$

Substitution and integration gives

$$
2 \pi \rho_{\infty} u_{m, c}{ }^{2} \beta_{c}^{2}\left(z+z_{o}\right)^{2} \psi_{2,1}=-2 \pi \tau_{w} \eta_{c}^{99} \beta_{c}\left(\frac{1}{2} z^{2}+z_{o} z\right)-\frac{\pi^{2}}{2} \eta_{c}^{99^{2}} \beta_{c}^{2}\left(z+z_{o}\right)^{2} f_{v o r t e x} \tau_{w}+M_{o}
$$

Solving for the centerline velocity (out of the head) gives

$$
u_{m, c}=\frac{c_{j}}{\beta_{c}} \sqrt{\frac{\rho_{o}}{\rho_{\infty}} \frac{N f_{N}}{8 \psi_{2,1}}-\frac{\tau_{w}}{\rho_{o} U_{o}^{2}} \frac{\rho_{o}}{\rho_{\infty}} \frac{\eta_{c}^{99} \beta_{c}}{\psi_{2,1}}\left(\frac{1}{2} \frac{z^{2}}{d_{o}^{2}}+\frac{z_{o}}{d_{o}} \frac{z}{d_{o}}+\frac{\pi \eta_{c}^{99} \beta_{c} f_{\text {vortex }}}{4}\left(\frac{z}{d_{o}}+\frac{z_{o}}{d_{o}}\right)^{2}\right)} \frac{U_{o} d_{o}}{z+z_{o}}
$$

In scaled form,

$$
\frac{u_{m, c}}{U_{o}}=\frac{c_{j}}{\beta_{c}} \sqrt{\frac{\rho_{o}}{\rho_{\infty}} \frac{N f_{N}}{8 \psi_{2,1}}-\frac{1}{\operatorname{Re}_{\tau}} \frac{\tau_{w}}{\tau_{o}} \frac{\rho_{o}}{\rho_{\infty}} \frac{\eta_{c}^{99} \beta_{c}}{\psi_{2,1}}\left(\frac{1}{2} \frac{z^{2}}{d_{o}^{2}}+\frac{z_{o}}{d_{o}} \frac{z}{d_{o}}+\frac{\pi \eta_{c}^{99} \beta_{c} f_{\text {vortex }}}{4}\left(\frac{z}{d_{o}}+\frac{z_{o}}{d_{o}}\right)^{2}\right)} \frac{1}{z / d_{o}+z_{o} / d_{o}}
$$

where this yield Reynolds number is based on a Bingham yield stress. The wall stress contains contributions from both viscous and yield stresses. A critical elevation may be identified where the velocity vanishes as 


$$
\frac{1}{2} \frac{z_{c}^{2}}{d_{o}{ }^{2}}+\frac{z_{c}}{d_{o}} \frac{z_{o}}{d_{o}}+\frac{\pi \eta_{c}^{99} \beta_{c} f_{\text {vortex }}}{2\left(2+\pi \eta_{c}^{99} \beta_{c} f_{\text {vortex }}\right)} \frac{z_{o}{ }^{2}}{d_{o}^{2}}-\frac{N f_{N}}{4 \eta_{c}^{99} \beta_{c}\left(2+\pi \eta_{c}^{99} \beta_{c} f_{\text {vortex }}\right)} \frac{\tau_{o}}{\tau_{w}} \operatorname{Re}_{\tau}=0
$$

The quadratic may then be solved as

$$
\frac{z_{c}}{d_{o}}=\sqrt{\frac{2}{2+\pi \eta_{c}^{99} \beta_{c} f_{\text {vortex }}} \frac{z_{o}^{2}}{d_{o}^{2}}+\frac{N f_{N}}{2 \eta_{c}^{99} \beta_{c}\left(2+\pi \eta_{c}^{99} \beta_{c} f_{\text {vortex }}\right)} \frac{\tau_{o}}{\tau_{w}} \operatorname{Re}_{\tau}}-\frac{z_{o}}{d_{o}}
$$

In the hypothetical absence of the virtual origin,

$$
\frac{1}{2}\left(\eta_{c}^{99} \beta_{c}\right)^{2}+\frac{1}{\pi f_{\text {vortex }}} \eta_{c}^{99} \beta_{c}-\frac{N f_{N}}{4 \pi f_{\text {vortex }} \frac{z_{c}^{2}}{d_{o}^{2}}} \frac{\tau_{o}}{\tau_{w}} \operatorname{Re}_{\tau}=0
$$

So that,

$$
\eta_{c}^{99} \beta_{c}=-\frac{1}{\pi f_{\text {vortex }}}+\sqrt{\frac{1}{\pi^{2} f_{\text {vortex }}^{2}}+\frac{N f_{N} d_{o}^{2}}{2 \pi f_{\text {vortex }} z_{c}^{2}} \frac{\tau_{o}}{\tau_{w}} \mathrm{Re}_{\tau}}
$$

which suggests qualitatively that a) the radius of the expanding jet may increase with the yield Reynolds number and with the number of PJMs in the vessel or b) conversely, as the Bingham yield stress increases, the radius of the jet decreases. This is consistent with the finding in Section 2.2 that the KB slurry had the smallest value of $\beta_{c}$ in Table 2.1. Given the importance of the virtual origin, this equation should not be used for quantitative prediction in this form.

Both expressions for the critical elevation (Eqs. (6.7) and (6.15)) depend on only five parameters: $z_{o} / d_{o}, \operatorname{Re}_{\tau}, \tau_{w} / \tau_{o}$, and the $\eta_{c}{ }^{99} \beta_{c}$ and $N f_{N}$ products. The second and fifth are independent of geometric scaling. Figure 6.1 compares both expressions to "cavern" heights experimentally measured in laponite. (Limiting elevations cannot be directly visualized in KB slurries.) When the "cavern" height is nondimensionalized on the nozzle diameter and plotted versus the yield Reynolds number based on the measured Bingham yield stress, the data generally overlap each other. Fit parameters summarized are in Figure 6.1. The figure shows reasonable agreement for both models with these parameters, differing primarily in the intercept. The agreement is reasonable given that the model does not account for elasticity and fracture or large unmixed particles observed during the experiments (see Appendix A). Nevertheless, the data collapse onto essentially a single curve.

Please note that the yield Reynolds number is not based on shear strength because the governing equations specify a Bingham yield parameter instead of a shear strength. 

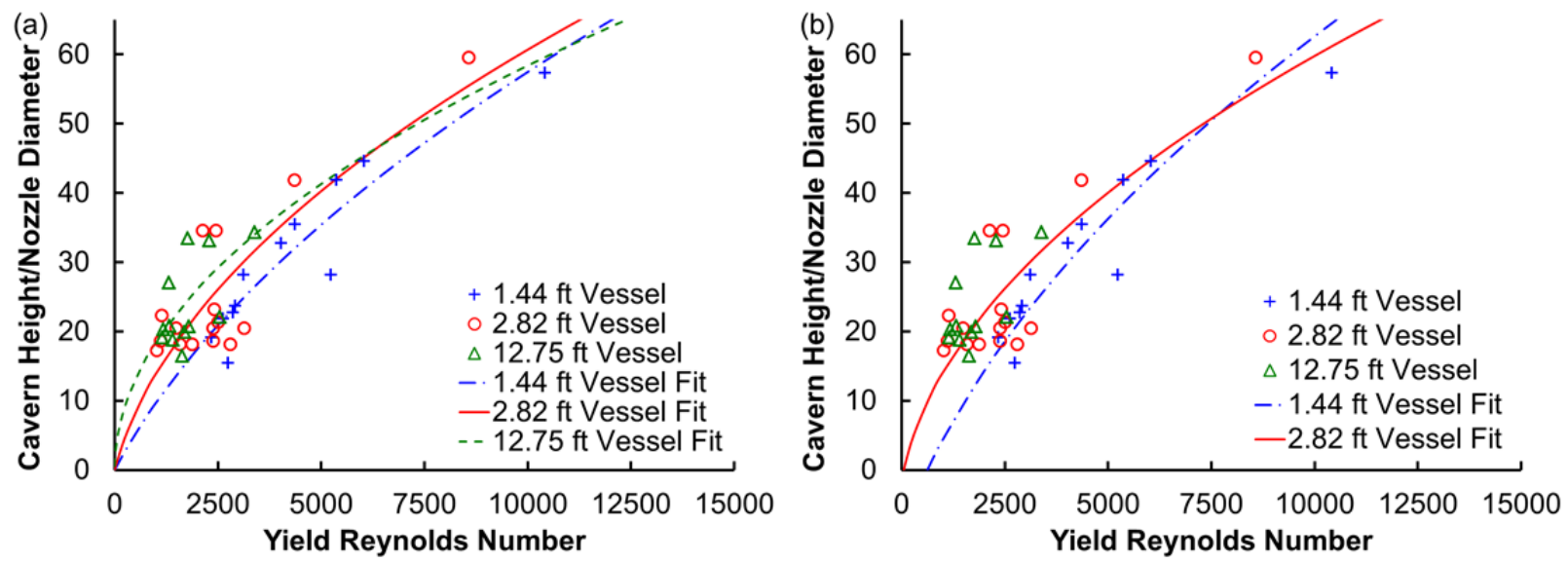

Figure 6.1. "Cavern" Height Data Scaled on Nozzle Diameter Versus Yield Reynolds Number with Best Fits to (a) Eq. (6.7) and (b) Eq. (6.15) with Fit Parameters in Table 6.1

Table 6.1 presents the parameters used to fit the curves in Figure 6.1. The product of $\eta_{c}{ }^{99} \beta_{c}$ cannot be separated into individual variables, but from the radial distribution measured in Section 2.3, one may anticipate $\beta_{c} \sim 0.06$ (Table 2.1) and $\eta_{c}^{99} \sim 1.6$ (Table 3.1) for $\eta_{c}^{99} \beta_{c} \sim 0.10$ for KB slurry. However, in laponite the fits for Eq. (6.7) significantly exceed this expectation. With Eq. (6.15), $\eta_{c}{ }^{99} \beta_{c}$ decreases by a factor of two so that the fit values lie within a factor of 2 to 4 of the actual values. This degree of variation is not surprising given that these values have not been directly measured in laponite (velocity data was taken but has not been reduced or analyzed in Bamberger et al. (2005)). In either case, this product appears to increase with vessel size (see Section 9). Remarkably, only the experiments in the $1.44 \mathrm{ft}$ vessel at Savannah River National Laboratory (SRNL) fit a value of $f_{\text {vortex }}>1$, suggesting that in the larger vessels the leading vortex did not extend significantly beyond the anticipated jet radius. The reasons for this finding and whether this is a vessel size effect are discussed below.

Table 6.1. Best Fit Parameters for "Caverns" Measured in Laponite

\begin{tabular}{lcccccccc}
\hline & Vessel & \multicolumn{2}{c}{ Eq. (6.7) } & \multicolumn{3}{c}{ Eq. (6.15) } & \multicolumn{2}{c}{ Eq. (6.27) } \\
\cline { 3 - 9 } Location & $(\mathrm{ft})$ & $\eta_{c}^{99} \beta_{c}$ & $z_{o} / d_{o}$ & $\eta_{c}{ }^{99} \beta_{c}$ & $f_{\text {vortex }}{ }^{(a)}$ & $z_{o} / d_{o}$ & $R_{\text {jet }} / d_{o}$ & $R_{\text {jet }}$ (inches) \\
\hline SRNL & 1.44 & 0.371 & 29.8 & 0.158 & 3.00 & 36.3 & $17.3 \pm 3.4$ & $7.48 \pm 1.46$ \\
APEL & 2.82 & 0.497 & 11.1 & 0.350 & 1.00 & 8.42 & $12.4 \pm 4.3$ & $10.7 \pm 3.8$ \\
336 & 12.75 & 0.733 & 0.00 & $\mathrm{IND}^{(\mathrm{b})}$ & $\mathrm{IND}^{(\mathrm{b})}$ & 0.00 & $9.51 \pm 2.61$ & $38.2 \pm 10.5$ \\
All & - & 0.768 & 3.21 & 0.455 & 1.00 & 2.84 & $13.0 \pm 4.7$ & - \\
\hline
\end{tabular}

(a) Confined to the domain of 1-3 inclusive given that this is the anticipate range of values for a leading vortex.

(b) These values were found to be mathematical indeterminate (IND) during the fitting because $z_{o} / d_{o}$ was zero.

\subsection{Confined Jets of Constant Radius}

Section 2.2 suggested that the radial velocity profile for the KB slurry may be more plug flow than Gaussian, which may confine and channel the turbulence. Rajaratnam and coworkers in Cai et al. (2012) considered sand jets entering laponite gel. They showed that when the jet velocity was sufficiently large, the jet was turbulent and dispersive. As the jet velocity dropped, the flow eventually took on the profile of a laminar tube of approximately constant diameter. This section considers the intermediate approximation of a turbulent jet of constant radius. Given that the jet spread remains rather modest for 
the KB slurry, this may not be a bad approximation. Shekarriz et al. (1997) argue that when a turbulent jet becomes confined, the turbulence increases by approximately a factor of 3.5 (see their Figure 2.6), which may enhance the persistence of jet momentum. Without channeling, the jet flow may decay faster. For a constant radius, the flow becomes akin to pipe flow, consistent with the arguments of Rajaratnam (1976, Chapter 8) for developed confined jets.
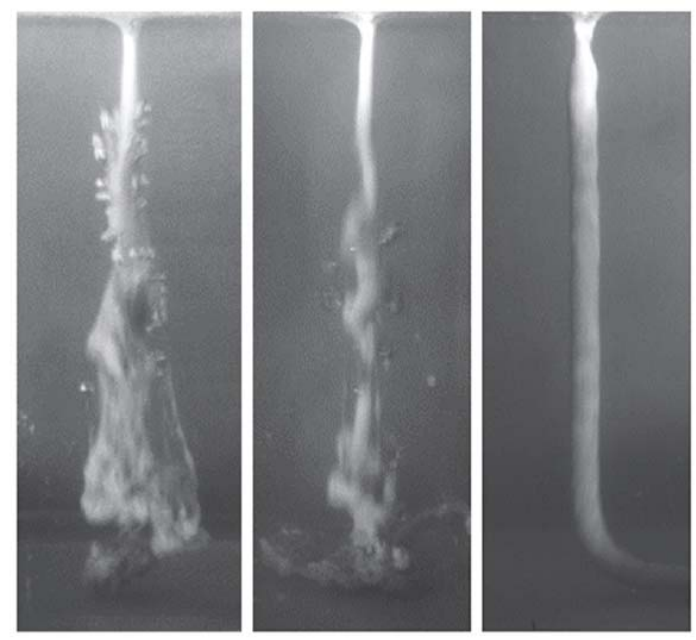

Figure 6.2. Sand Jet in Laponite at Decreasing Entrance Velocities (left to right). Original caption: "Fig. 2. Example images of deformation types I to III of sand jets in Laponite gel." Reprinted with permission from Cai et al., Copyright 2012, Chinese Society of Theoretical and Applied Mechanics and American Institute of Physics.

In Section 4, the sum of stresses using a Prandtl mixing length model for turbulence was given in Eq. (4.15) (repeated below) as

$$
\tau_{\text {lam }}+\tau_{\text {turb }}=\tau_{o}\left(1-\operatorname{Exp}\left[-m\left|\frac{\partial u}{\partial r}\right|\right]\right)+\mu_{o} \frac{\partial u}{\partial r}+\rho_{\infty} l^{2}\left|\frac{\partial u}{\partial r}\right| \frac{\partial u}{\partial r}
$$

Near a pipe wall, Masuyama et al. (1980) cite the work of Hanks and give the mixing length for a Bingham fluid as

$$
l=0.36 y\left(1-\operatorname{Exp}\left[-\frac{y}{R} \frac{\alpha\left(1-\alpha_{c}^{\prime} / \alpha_{M}\right)}{22 B^{\prime}(H e)}\right]\right)
$$

where $y$ is the distance from the wall, $\alpha_{M}$ is a Reynolds number based on the Bingham consistency and wall velocity, $\alpha_{c}$ ' is a constant discussed in their article, and $B^{\prime}$ is an empirical correction factor

$$
B^{\prime}(H e)=1+\frac{0.00352 H e}{(1+0.000504 H e)^{2}}
$$

with $\mathrm{He}$ as the Hedstrom number, 


$$
H e=\frac{4 \rho \tau_{o} R_{j e t}{ }^{2}}{\mu_{o}{ }^{2}}
$$

At the wall $(y=0)$, Eq. (6.18) shows the mixing length vanishes. This is consistent with the well-known laminar sublayer arguments near flat walls and the discussion of Deen $(2012$, p. 504), who argues that velocity fluctuations must vanish near a wall. Alternatively, even in approximate confinement where the mixing length may not completely vanish, the radial profiles in Figure $2.4 \mathrm{~b}$ permit fitting by functions that require the derivative of the axial velocity with respect to radius to vanish infinitely far from the centerline of the jet. In either case, the turbulent stresses vanish.

The viscous stresses are more nuanced. If the axial velocity profile asymptotes to zero so that its first derivative also asymptotes to zero, then the viscous stresses vanish at $R_{j e t}$. Alternatively, the magnitude of the viscous stress may be compared to the magnitude of the yield stress. The ratio of viscous stresses to yield stresses (akin to the inverse of the Bingham number) may be extracted from Eqs. (4.17) and (4.18) as

$$
\frac{\text { viscous stresses }}{\text { yield stresses }}=\frac{\operatorname{Re}_{\tau}}{\beta_{c} \operatorname{Re}_{o}}=\frac{\mu_{o} U_{o}}{\beta_{c} \tau_{o} d_{o}}
$$

which is typically order one for a $30 \mathrm{~Pa}, 30 \mathrm{mPa}$ s simulant with a nozzle velocity and diameter of $12 \mathrm{~m} / \mathrm{s}$ and 4 inches and values of $\beta_{c}$ in Table 2.1. In either case, if the jet is rigidly confined, then following Eq. 5.3-8 of Bird et al. (2002), the yield stress and viscous stress may be combined into an equivalent wall stress. Eq. (4.15) reduces to

$$
\tau_{\text {lam }}+\tau_{\text {turb }}=-\tau_{w}
$$

in the limit of large $m$, where the minus sign indicates that the wall stresses act on the fluid. Eq. (6.1) then governs with $R_{j e t}$ as a constant that may be factored out of the integral. Performing the integration gives

$$
2 \pi \rho_{\infty} u_{m, c}{ }^{2} \beta_{c}{ }^{2}\left(z+z_{o}\right)^{2} \psi_{2,1}=-2 \pi \tau_{w} R_{j e t} z+M_{o}
$$

from which the axial velocity may be determined with Eq. (3.1) as

$$
u_{m, c}=\sqrt{\frac{N f_{N}}{8 \psi_{2,1} \beta_{c}^{2}} \frac{\rho_{o}}{\rho_{\infty}}-\frac{\rho_{o}}{\rho_{\infty} \beta_{c}^{2} \psi_{2,1}} \frac{1}{\operatorname{Re}_{\tau}} \frac{\tau_{w}}{\tau_{o}} \frac{R_{j e t}}{d_{o}} \frac{z}{d_{o}} \frac{d_{o} U_{o}}{z+z_{o}}}
$$

Scaling finds

$$
\frac{u_{m, c}}{U_{o}}=\sqrt{\frac{N f_{N}}{8 \psi_{2,1} \beta_{c}{ }^{2}} \frac{\rho_{o}}{\rho_{\infty}}-\frac{\rho_{o}}{\rho_{\infty} \beta_{c}{ }^{2} \psi_{2,1}} \frac{1}{\operatorname{Re}_{\tau}} \frac{\tau_{w}}{\tau_{o}} \frac{R_{j e t}}{d_{o}} \frac{z}{d_{o}} \frac{1}{z / d_{o}+z_{o} / d_{o}}}
$$

The critical elevation at which the jet would fail if fully turbulent (in the absence of further laminar transition) may be determined where the local axial velocity falls to zero. Then, 


$$
\frac{z_{c}}{d_{o}}=\frac{N f_{N}}{8} \frac{d_{o}}{R_{j e t}} \frac{\tau_{o}}{\tau_{w}} \operatorname{Re}_{\tau}
$$

This equation shows that the critical elevation depends linearly on the yield Reynolds number instead of to the $1 / 2$ power for the expanding jet. Support for linear fits is found in Figures 5.3 through 5.6 of Bamberger et al. (2005), which plot the "cavern" height nondimensionalized on the tank diameter versus the yield Reynolds number based on a shear strength. This data set, using their average velocity (most similar to the averaging done elsewhere in this report) and based on the Bingham yield stress alone, is replotted in Figure 6.3a without an intercept as indicated by Eq. (6.26). The fits are not bad if uninspiring. ${ }^{1}$

To explain the scatter, Eq. (6.26) may be rearranged to determine the jet radius as

$$
\frac{R_{j e t}}{d_{o}}=\frac{N f_{N} d_{o} \operatorname{Re}_{\tau}}{8 z_{c}} \frac{\tau_{o}}{\tau_{w}}
$$

Figure $6.3 \mathrm{~b}$ shows that when the yield Reynolds number is zero either due to negligible nozzle velocities or large yield stresses, the jet radius begins with a negligible radius. As the nozzle velocity increases or the yield stress decreases, the jet radius increases. When the velocity increases further, the viscous forces become more important and begin to dominate; see Eq. (6.21). Figure 6.3c replots the scaled jet radius versus the ratio of the yield Reynolds number to the jet Reynolds number, which represents the ratio of viscous to yield forces. This figure shows that there is a change in slope and magnitude of the scaled jet radius around particular values of $\mathrm{Re}_{\tau} / \mathrm{Re}_{\mathrm{o}}$. The precise transition where the viscous forces become generally significant remains unclear because the factor of $1 / \beta_{c}$ in the denominator of Eq. (6.21) remains unknown. Nevertheless, Table 2.1 shows that $\beta_{c} \sim 0.05-0.20$ typically, so that dividing the $x$-axis by $\beta_{c}$ would bring the transition in behavior to around a value of 1 , which implies that the curves may collapse around that value.

Importantly, the value of $\operatorname{Re}_{\tau} / \beta_{c} \operatorname{Re}_{o} \sim O(1)$, suggesting that viscous forces may be influential and the jet Reynolds number with its dependence on geometric scale may not be negligible. By direct implication, a velocity of $12 \mathrm{~m} / \mathrm{s}$ in the $8 \mathrm{ft}$ vessel may or may not be a velocity of $12 \mathrm{~m} / \mathrm{s}$ in the $16 \mathrm{ft}$ vessel, though how much variation may be reasonably expected requires additional analysis. Please see additional analysis by Wells et al. (2015) for quantitative analysis of direct scaling based on historical data.

\footnotetext{
${ }^{1}$ The $\mathrm{R}^{2}$ value here does not denote the square of the correlation coefficient but represents one minus the sum of the square of the errors for the trendline divided by the sum of the square of the errors for a horizontal line at the elevation of the arithmetic average, both relative to the data. In this sense, a negative $\mathrm{R}^{2}$ value only indicates that a horizontal line at the elevation of the arithmetic average provides less deviation than the trendline.
} 

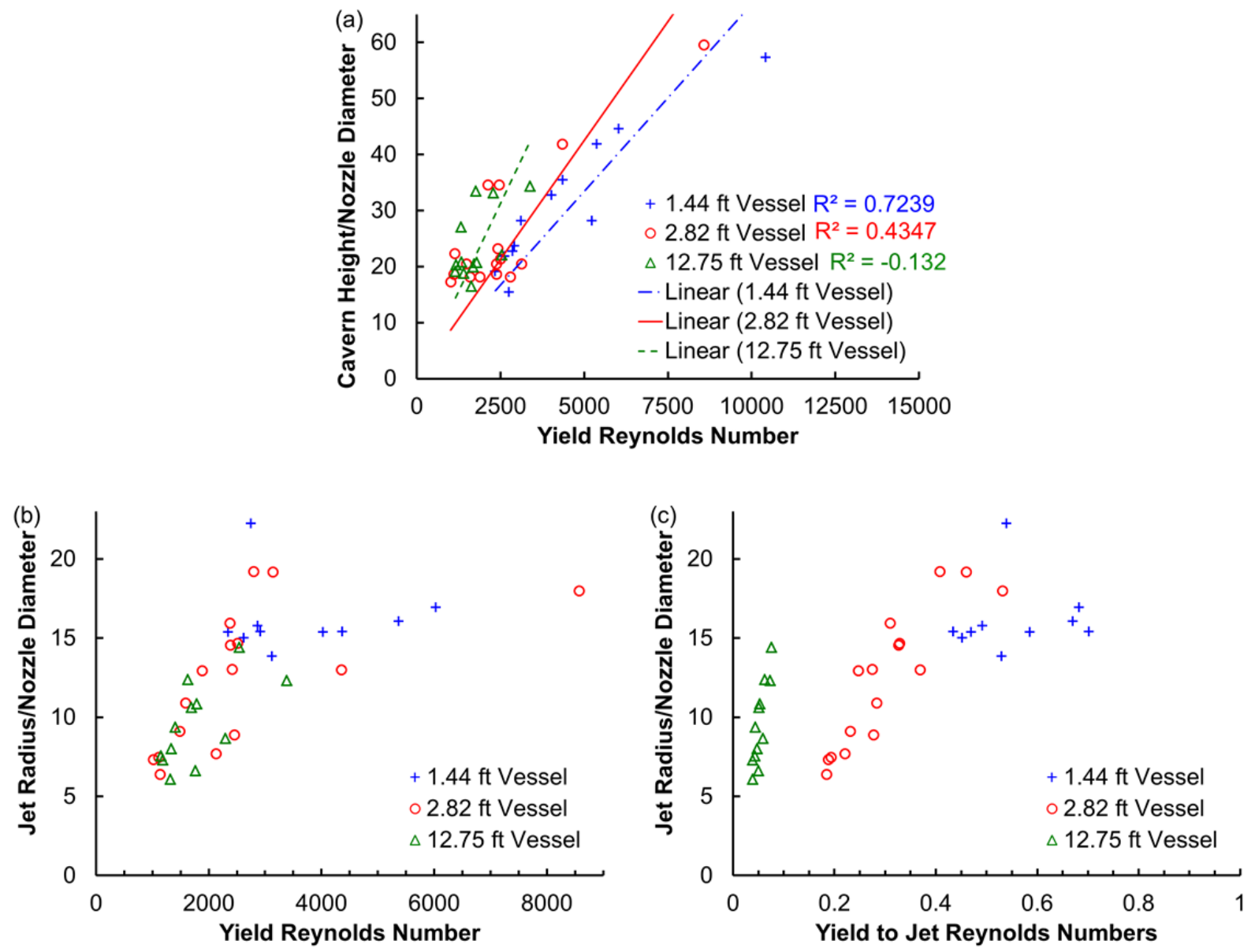

Figure 6.3. (a) "Cavern" Height Versus Yield Reynolds Number, (b) $R_{j e t}$ Nondimensionalized on the Nozzle Diameter versus Yield Reynolds Number, and (c) $R_{j e t}$ Nondimensionalized on the Nozzle Diameter versus the Ratio of the Yield Reynolds Number to the Jet Reynolds Number for Laponite Gels at Three Vessel Sizes

Table 6.2. Best Fit Parameters for "Caverns" Measured in KB Slurry ${ }^{(\mathrm{a})}$

\begin{tabular}{lccccccc}
\hline Test & Location & $z_{c} / d_{o}$ & $\mathrm{Re}_{\tau}$ & $\mathrm{Re}_{o}$ & Viscous/Yield Forces & $R_{\text {jet }} / d_{o}$ & $R_{\text {jet }}$ (inches) \\
\hline S-16 & SRNL & 14.1 & 3167 & 4267 & 13.47 & 12.2 \\
M-20 & APEL & 13.4 & 1225 & 4674 & 4.75 & 11.5 & 9.92 \\
M-21 & APEL & 13.5 & 888 & 7941 & 2.030 & 8.20 & 7.10 \\
L-18 & 336 & 13.5 & 874 & 30386 & 0.522 & 8.10 & 32.5 \\
L-19 & 336 & 13.4 & 1375 & 35423 & 0.705 & 12.8 & 51.4 \\
L-20 & 336 & 13.4 & 1361 & 35235 & 0.701 & 12.7 & 50.9 \\
\hline
\end{tabular}

(a) Calculated using Eq. (6.27) with $N f_{N}=1, \tau_{o} / \tau_{w}=1, \beta_{c}=0.0551$ from Table 2.1 for KB slurry in the $12.75 \mathrm{ft}$ vessel. 
When the viscous stress is not negligible, the wall stress no longer equals the Bingham yield stress but has contributions from the viscous term. The wall stress may be approximated by the Fanning friction factor in a Bingham fluid, $f_{B}$, as

$$
\tau_{w}=\frac{f_{B}}{2} \rho_{\infty} u_{m, c}^{2}
$$

Kuhn et al. (2013) in Section E.6.2.1.2.2 provide a model for the friction factor of a Bingham fluid. They give

$$
f_{B}=\left(f_{L}^{m_{B}}+f_{T}^{m_{B}}\right)^{1 / m_{B}}
$$

where

$$
m_{B}=1.7+\frac{40000}{\mathrm{Re}_{B}}
$$

with

$$
\operatorname{Re}_{B}=\frac{2 \rho u_{m, c} R_{j e t}}{\mu_{o}}
$$

The laminar friction factor is given by

$$
f_{L}=\frac{16}{\operatorname{Re}_{B}}\left[1+\frac{1}{6} \frac{H e}{\operatorname{Re}_{B}}-\frac{1}{3} \frac{H e^{4}}{f_{L}^{3} \operatorname{Re}_{B}^{7}}\right]
$$

and the turbulent friction factor is

$$
f_{T}=10^{\beta} \operatorname{Re}_{B}^{-0.193}
$$

with

$$
\beta=-1.378\left(1+0.146 \operatorname{Exp}\left[-0.29 \cdot 10^{-5} \operatorname{Re}_{B}\right]\right)
$$

These equations from Kuhn et al. (2013) must be solved iteratively.

Figure 6.4a shows with these equations that increasing the Hedstrom number delays the turbulent-to-laminar transition. Similarly, Masuyama et al. (1980) experimentally determined the Fanning friction factor for kaolin slurries. Their data in Figure $6.4 \mathrm{~b}$ also show a delay in the laminar-turbulent transition with increasing He. In either case, for a jet radius of 0.10-1.0 m moving at a velocity of $0.1-1.0 \mathrm{~m} / \mathrm{s}$ in a Bingham material with $\rho=1200 \mathrm{~kg} / \mathrm{m}^{3}, \tau_{o}=30 \mathrm{~Pa}$ and $\mu_{o}=30 \mathrm{mPa} \cdot \mathrm{He}=1.6 \cdot 10^{6}$ to $1.6 \cdot 10^{8}$, and $\mathrm{Re}_{\mathrm{B}}=8.0 \cdot 10^{2}-8.0 \cdot 10^{4}$. Both panels indicate that as the jet speed decreases, the jet becomes laminar for the Bingham Reynolds numbers of interest. This prediction of laminar flow comports with 
the qualitative trend in Figure 2.12 and should not be surprising because the viscous stresses are of the same order of magnitude as the yield stresses. Laminar flows are examined next.
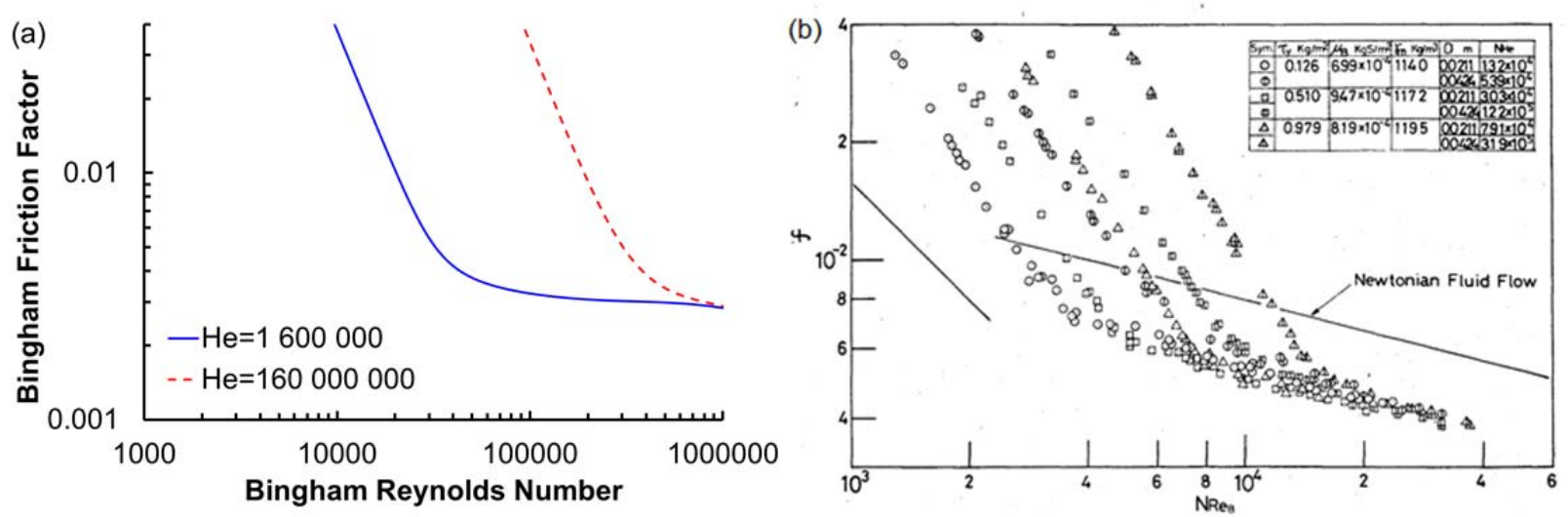

Figure 6.4. Fanning Friction Factor for a Bingham Plastic Versus the Bingham Reynolds Number (a) for $\mathrm{He}=1.6 \cdot 10^{6}$ and $1.6 \cdot 10^{8}$ and (b) for Experimental Data Using Kaolin Slurries from Masuyama et al. (1980). Original Caption: "Fig. 2 Relationship between $f$ and $N_{R e B}$ for kaolin slurries." Note the offset in axes between panels. Reprinted with permission.

\subsection{Bingham Flow in Constant Radius Laminar Tubes}

Although Section 4.3 suggests that the flow may converge, the last upwell considered herein is a completely laminar flow of constant diameter. Converging flows are known to suppress the instability that leads to turbulence. This flow configuration is supported by Figure 2.12, which shows a complete absence of turbulence at the upper probe in $8 \mathrm{ft}$ testing in KB slurries at modest velocities, and by Figure 6.2, which depicts laminar flow of a sand jet in laponite. Chatzimina et al. (2007) examined the cessation of laminar annular flows with Bingham plastic rheology. ${ }^{1}$ Both Chatzimina et al. (2007) and Plawsky (2001) give the well-known solution for the velocity profile of a Bingham plastic in a pipe at low Reynolds number in the notation herein as

$$
u=\frac{R_{j e t}{ }^{2}}{4 \mu_{o}}\left(-\frac{\partial p}{\partial z}\right)\left[1-\frac{r^{2}}{R_{j e t}{ }^{2}}\right]-\frac{\tau_{o} R_{j e t}}{\mu_{o}}\left(1-\frac{r}{R_{j e t}}\right)
$$

in the sheared region between $r_{c}$ and $R_{\text {jet }}$. The region between 0 and $r_{c}$, given by setting the derivative of the velocity profile to zero as

$$
r_{c}=\frac{2 \tau_{o}}{\left(-\frac{\partial p}{\partial z}\right)}
$$

contains unsheared material moving at a constant velocity, $u_{c}$,

\footnotetext{
${ }^{1}$ Although not a focus of this report, the transient analysis of Chatzimina et al. (2007) may be used to assess (with modification for bubbly flow) the presence of the spargers and the additional shear they impose on the central upwell.
} 


$$
u_{c}=\frac{R_{j e t}^{2}}{4 \mu_{o}}\left(-\frac{\partial p}{\partial z}\right)\left[1-\frac{r_{c}^{2}}{R_{j e t}^{2}}\right]-\frac{\tau_{o} R_{j e t}}{\mu_{o}}\left(1-\frac{r_{c}}{R_{j e t}}\right)
$$

The velocity profile may then be normalized to give

$$
\frac{u}{u_{c}}=\frac{\tau_{o} R_{j e t}}{\mu_{o} u_{c}}\left[\frac{R_{j e t}}{2 r_{c}}\left(1-\frac{r^{2}}{R_{j e t}^{2}}\right)-\left(1-\frac{r}{R_{\text {jet }}}\right)\right]
$$

subject to the constraint that the normalized profile must be continuous.

This normalized dependence contrasts with the $1 / 7^{\text {th }}$ power law expected of turbulent flow given by Bird et al. (2002) as

$$
\frac{u}{u_{m, c}}=\left(1-\frac{r}{R_{j e t}}\right)^{1 / 7}
$$

Figure 6.5 compares the laminar, turbulent, and Gaussian axial velocity profiles to experimental data from Figure $2.4 \mathrm{~b}$ for a KB slurry from Bamberger et al. (2005). The velocity profile shows that the laminar profile matches the data well, even though the estimated Reynolds numbers substantially exceed unity. The agreement justifies exploring the implication of the low Reynolds number laminar velocity profile even though the Reynolds number is not necessarily low.

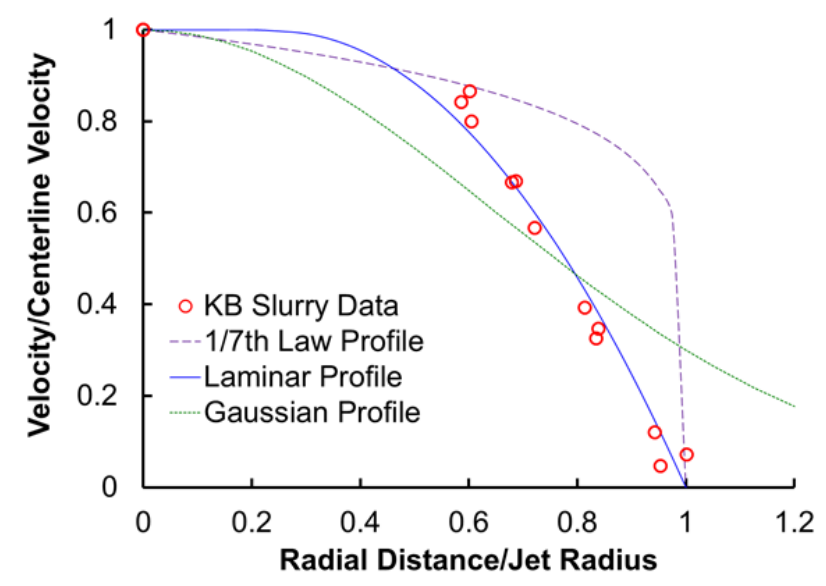

Figure 6.5. Axial Velocity Profiles Normalized on the Centerline Velocity Versus Radial Position Normalized on the Jet Radius Determined by Linear Fitting. Experimental data from Figure $2.4 \mathrm{~b}$ compared to the $1 / 7^{\text {th }}$ law, laminar profile, and Gaussian profiles. Best fit laminar velocity profile constants are $r_{c} / R_{j e t}=0.245$ and $\tau_{o} R_{j e t} / \mu_{o} u_{c}=0.857$. 
Eq. (6.1) with a constant radius becomes

$$
\int_{0}^{R_{j e t}} 2 \pi \rho_{\infty} u^{2} r d r=-2 \pi \tau_{w} \int_{0}^{z} R_{j e t} d z+M_{o}
$$

This time, the velocity in the first term arises from the well-known solutions for laminar flow in a Bingham plastic at steady state. With Eq. (3.1),

$$
2 \pi R_{\text {jet }}{ }^{2} \rho_{\infty} u_{m, c}{ }^{2} \psi_{B}=-2 \pi \tau_{w} R_{j e t} z+\frac{\pi}{4} N f_{N} \rho_{o} U_{o}{ }^{2} d_{o}{ }^{2}
$$

where

$$
\psi_{B}=\frac{1}{2} \frac{r_{c}{ }^{2}}{R_{j e t}{ }^{2}}+\left(\frac{\tau_{o} R_{j e t}}{\mu_{o} u_{c}}\right)^{2} \int_{r_{c} / R_{j e t}}^{1}\left[\frac{R_{j e t}}{2 r_{c}}\left(1-\frac{r^{2}}{R_{j e t}{ }^{2}}\right)-\left(1-\frac{r}{R_{j e t}}\right)\right]^{2} \frac{r}{R_{j e t}} d \frac{r}{R_{j e t}}
$$

Integration finds

$$
\psi_{B}=\frac{1}{2} \frac{r_{c}{ }^{2}}{R_{j e t}{ }^{2}}+\left(\frac{\tau_{o} R_{j e t}}{\mu_{o} u_{c}}\right)^{2} \frac{\left(1-\frac{r_{c}}{R_{j e t}}\right)^{5}\left(5+11 \frac{r_{c}}{R_{j e t}}\right)}{120 \frac{r_{c}{ }^{2}}{R_{j e t}{ }^{2}}} \approx 0.222
$$

for the best fit values used to fit the data in Figure 6.5. Solving for $u_{m, c}$ finds

$$
u_{m, c}^{2}=\frac{N f_{N} \rho_{o} U_{o}^{2} d_{o}^{2}}{8 R_{j e t}^{2} \rho_{\infty} \psi_{B}}-\frac{\tau_{w}}{R_{j e t} \rho_{\infty} \psi_{B}} z
$$

or in scaled form,

$$
\frac{u_{m, c}^{2}}{U_{o}^{2}}=\frac{N f_{N}}{8 \psi_{B}} \frac{\rho_{o}}{\rho_{\infty}} \frac{d_{o}^{2}}{R_{j e t}^{2}}-\frac{1}{\operatorname{Re}_{\tau} \psi_{B}} \frac{\tau_{w}}{\tau_{o}} \frac{\rho_{o}}{\rho_{\infty}} \frac{d_{o}}{R_{j e t}} \frac{z}{d_{o}}
$$

This equation anticipates that a plot of the square of the scaled velocity versus the scaled elevation may comprise a straight line. Because the yield Reynolds number is greater than one, the slope should be quite shallow. The intercept depends strongly on $R_{j e t} / d_{o}$, with wider jets decreasing the intercept. Although the parameters share several factors, the intercept depends uniquely on the number and spatial arrangement of PJMs. The slope depends uniquely on the yield Reynolds number and a ratio of the wall stress to the Bingham yield stress.

Figure 6.6 shows that this proposed scaling does remarkably well. Indeed, the curves are essentially flat. Fit parameters are summarized in Table 6.3. The curves were prepared with peak average velocities 
for the $2.82 \mathrm{ft}$ and $12.75 \mathrm{ft}$ vessels. Reprocessing the raw data (unavailable) with average velocities would raise these curves in Figure 6.6 and bring the predicted elevation of breakthrough into closer agreement with experiment. Figure 2.5 suggests that the jet width expands slightly with elevation. This model operates under a lubrication approximation and does not account for this modest expansion. Modest improvement in predictions may be expected by including this effect.

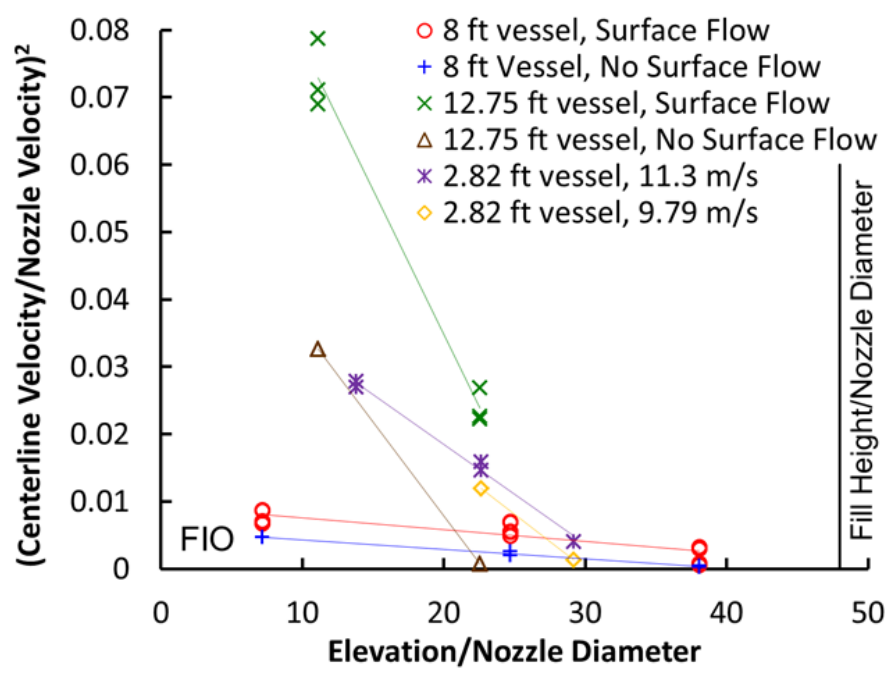

Figure 6.6. The Square of the Ratio of the Centerline Velocity to the Nozzle Velocity Versus the Elevation Scaled on Nozzle Diameter. Nozzle velocity for $2.82 \mathrm{ft}$ and $12.75 \mathrm{ft}$ vessels was the peak average velocity (larger than average defined in Sections 2.1 and 2.4). Data from the $2.82 \mathrm{ft}$ vessel taken off center, which reduces values on the y-axis by a factor of $f_{c}^{2}$, and are included only to inform consideration of predicted linearity. The vertical line represents the nominal fill height of the 8 or $16 \mathrm{ft}$ vessels; the other vessels had a dimensionless fill height of 29 to 36 .

Table 6.3. Laminar Profile Fits

\begin{tabular}{lccccccc}
\hline & $\begin{array}{c}\text { Nozzle } \\
\text { Velocity } \\
(\mathrm{m} / \mathrm{s})\end{array}$ & $\begin{array}{c}\text { Nozzle } \\
\text { Diameter } \\
\text { (inches) }\end{array}$ & Slope & Intercept & $R_{\text {jet }} / d_{o}^{(\text {a) }}$ & $\begin{array}{c}R_{\text {jet }} \\
(\text { inches })\end{array}$ & $z_{c} / d_{o}$ \\
\hline $8 \mathrm{ft}^{(\mathrm{b})}$ & 8 & 1.94 & $-1.38 \cdot 10^{-4}$ & 0.00566 & 14.1 & 27.4 & 41.0 \\
$8 \mathrm{ft}^{(\mathrm{b})}$ & 12 & 1.94 & $-1.20 \cdot 10^{-4}$ & 0.00789 & 11.9 & 23.2 & 65.6 \\
$12.75 \mathrm{ft}$ & $8.2-8.6^{(\mathrm{c})}$ & 4.0 & $-4.27 \cdot 10^{-3}$ & 0.120 & 2.16 & 8.65 & 28.2 \\
$12.75 \mathrm{ft}$ & $5.48-5.50^{(\mathrm{c})}$ & 4.0 & $-2.78 \cdot 10^{-3}$ & 0.0635 & 2.98 & 11.9 & 22.8 \\
\hline
\end{tabular}

(a) Asserts $\psi_{B}=0.222, \tau_{w} / \tau_{o}=1$, and $\rho_{o} / \rho_{\infty}=1$ for all conditions, though these have not been measured generally.

(b) FIO.

(c) Uses peak average velocity in the $12.75 \mathrm{ft}$ vessel. Using the average nozzle velocity would increase intercept and critical elevation estimates.

An expression for the critical elevation arises when the scaled velocity goes to zero as

$$
\frac{z_{c}}{d_{o}}=\frac{N f_{N}}{8} \frac{d_{o}}{R_{j e t}} \frac{\tau_{o}}{\tau_{w}} \operatorname{Re}_{\tau}
$$


Again, when the jet is not expanding, the critical elevation depends linearly on the yield Reynolds defined on the Bingham yield stress. The critical elevation is determined by a balance of competing factors that control the slope and intercept. During design stages, the intercept may be determined by selecting $N f_{N}$, but during operations, the slope may be determined by selecting the $\operatorname{Re}_{\tau}$ or, when $\operatorname{Re}_{\tau}$ is sufficiently large, by selecting $\mathrm{Re}_{\mathrm{B}}, \mathrm{He}$, and $\mathrm{Re}_{\mathrm{o}}$. The inclusion of these dimensionless numbers introduces geometric scale dependence through $\mathrm{Re}_{\mathrm{o}}$ of unknown magnitude.

Derivations to explore the influence of the jet Reynolds number and the radial variation of the jet remain straightforward but intricate. Two solutions are necessary: one for turbulent flows and one for laminar flows. For the turbulent flows, energy balances and momentum balances must be coupled because the energy balance determines the radial spread of the jet (see Section 4.3). This allays the need to artificially distinguish between expanding and confined jets as in Section 6.1 versus 6.2. Both conservation equations must be integrated radially outward from the centerline of the jet. The outer limit of integration may be determined where the fluid shear stress equals the Bingham yield stress, which in turn defines the radial derivative of the axial velocity. This leads to a coupled pair of ordinary differential equations, which may then be solved with the usual methods. The laminar flow solution presented here may be modified to account for a slowly changing radius as shown in Figure 2.4 using a lubrication analysis. The transition between flows may be approximated by the friction factor analysis built on the work of Masuyama et al. (1980) or preferably with entropy considerations as presented by Bejan et al. (2014).

The estimated diameter has direct implications for surface flow of the slurry. For example, both the Froude number and the fountain height, which induces the pressure driven flow across the solidified slurry surface, scale on this diameter. Indeed, this is the crucial dimension in the problem, without which mode three flow as defined by Bamberger et al. (2005) simply could not be analyzed accurately. (The non-Newtonian mixing modes are (I) cavern only; (II) breakthrough with stagnant zones; (III) breakthrough with slow peripheral movement; (IV) all turbulent movement.) For example, for the $8 \mathrm{ft}$ vessel with a nozzle velocity of $12 \mathrm{~m} / \mathrm{s}$, the centerline velocity of a surface at $z / d_{o}=48$ decays to $0.55 \mathrm{~m} / \mathrm{s}$. With a density ratio of $10^{3}$, characteristic of water emerging into air, the Froude number is approximately 0.0051 . Srinarayana et al. (2008) review axisymmetric correlations to find that the turbulent steady-state fountain height in the notation herein is $2 C_{\text {fountain }} R_{j e t} \mathrm{Fr}$ where $C_{\text {fountain }}$ ranges from 2.05 to 2.74 , which gives a fountain height of approximately 0.49 to 0.65 inches; start up fountain heights may be larger. Other models may produce other answers. This height induces the pressure gradient that drives the transient lateral flow of the Bingham fluid across the surface with or without the imposition of surface waves as described by Lamb (1945). The flow is transient where both curvature and interfacial tension effects may influence the radially spreading fluid. Additionally, multiple hydrodynamic instabilities, including free surface and jet instabilities, influence the fluid flow as the jet approaches the surface and as the emerging fluid flows and ripples across the upper surface, the full analysis of which, though imminently tractable, lies outside the scope of this upwell analysis (Bouchet et al. 2002;

Srinarayana et al. 2008).

\subsection{Summary}

This section considers the influence of non-Newtonian fluids that have an actual yield stress (not simply an extrapolated yield stress from other non-Newtonian rheologies). Three cases were evaluated: a turbulent dispersive jet (c.f., Eqs. 6.5, 6.7, 6.13, and 6.15), a confined turbulent jet (Eqs. 6.25 and 6.26), 
and confined laminar flow (Eqs. 6.38, 6.45, and 6.46). The section shows that the radial distribution of axial velocities is a primary variable and that the viscous forces that depend on nozzle diameter cannot be neglected (Eq. 6.21). Of the three cases, the laminar flow case reasonably describes the radial profile (Figure 6.5), provides the best fit to velocity profiles in multiple vessels (Figure 6.6), and serves as the most conservative case.

The velocity distributions predicted feed directly into estimates of the local viscosity. The local effective viscosity may be described by the Bingham-Papanastasiou model (see Figure 2.6 and Eq. 4.15) as

$$
\mu_{e f f}=\mu_{o}+\frac{\tau_{o}}{\left|\frac{\partial u}{\partial r}\right|}\left(1-\operatorname{Exp}\left[-m\left|\frac{\partial u}{\partial r}\right|\right]\right)
$$

or for the Bingham plastic model

$$
\mu_{e f f}=\mu_{o}+\frac{\tau_{o}}{\left|\frac{\partial u}{\partial r}\right|}
$$

which show that the radial distribution of velocities is essential in determining the effective local viscosity. For small (e.g., $<1 \mathrm{~cm}$ ) spherical bubbles in the non-inertial limit (i.e., those trapped by yield stresses), the bubble (and gas) release rate through the central upwell (the primary flow channel to the surface) is, therefore, governed primarily by the local velocity distribution.

This analysis is insufficient for complete prediction of the velocity profiles because the elevation at which the flow transitions from turbulent at the base to laminar at higher elevations remains unknown and may or may not scale with vessel diameter. Even though the viscous forces cannot be neglected, the influence of viscosity has not been quantified herein, and momentum and energy coupling has not been completed. 


\subsection{Jet Celerity}

Analysis thus far has focused on the steady-state conditions after the initial jet front (and ring vortex) has passed. ${ }^{1}$ However, the rate at which a jet propagates may also affect whether the flow achieves surface flow or not. The rate at which the leading vortex of a jet moves is called jet celerity (i.e., the rapidity or swiftness of jet front penetration). Consider a jet that has passed the lowest probe in a vessel. A few moments later it passes the next probe and then moments after that it passes the third as it rises in elevation. By knowing the time at which the jet passes each probe, a plot of the elevation of the jet front versus time may be constructed. Nondimensionalizing the elevation on the nozzle diameter and the time on the jet time, $d_{o} / U_{o}$, collapses the curves, and the constants in a celerity equation may then be determined.

Sangras and Faeth (1999) give the celerity equation for a free turbulent primary flow as

$$
\frac{z}{d_{o}}=\alpha\left(\frac{t}{d_{o} / U_{o}}\right)^{n}
$$

where $\alpha$ is the celerity constant and $n$ is the celerity exponent. They show that for primary jets in the absence of solids $n=1 / 2$ for jets and $n=1 / 4$ for puffs. A puff is a jet-like flow that continues to propagate after flow at the nozzle has been turned off. Similarly, Witze (1980, see Eq. 27) finds a consensus in the literature around the use of $t-t_{\text {ref }} \sim\left(z_{\text {tip }}+z_{\text {ref }}\right)^{2}$ to describe jet celerity, where $z_{\text {tip }}$ is the instantaneous elevation of the jet tip or penetration front and $t_{\text {ref }}$ and $z_{\text {ref }}$ are fitting parameters. In either case, knowledge of upwell celerity curves in conjunction with the temporal analysis of bottom motion enable prediction of whether surface flow may be achieved.

To place the analysis below in context, two experiments are insightful. First, for the six-part simulant in Table 2.2, the velocity at the seam is always vigorous and turbulent (Figure 7.1). However, at higher elevations, the celerity of the jet may be attenuated. At $U_{o}=9.5 \mathrm{~m} / \mathrm{s}$ in Figure 7.1a, the jet gradually arrives at the middle probe and fails to reach the upper probe during the drive time. At $10.5 \mathrm{~m} / \mathrm{s}$, the jet arrives at the middle probe more quickly and in the last couple of seconds reaches the upper probe. At $11.5 \mathrm{~m} / \mathrm{s}$, the jet velocity gradually grows at upper probe, but surface flow is not observed until $12 \mathrm{~m} / \mathrm{s}$, though the flow is fast enough to reach the upper probe. By $15 \mathrm{~m} / \mathrm{s}$, the jet shows excellent celerity at all probe levels.

The gradual penetration of the jet at intermediate velocities suggests that jet celerity is an essential factor in achieving surface flow. Indeed, had the drive time been longer (e.g., at $10.5 \mathrm{~m} / \mathrm{s}$ ), the flow may have reached the upper probe and perhaps the upper surface. This is a significant result because it indicates that transient behavior may be important in understanding and predicting surface flow, cloud height, and "cavern" height. This is commensurate with the arguments of Kuhn et al. (2013), who indicate in their Section 9, Eq. 9.5, that the rate of cloud height rise is unconstrained but by time, which leads to their characteristic mixing time scales for plane jets. The cloud height then may not be a static, steady-state phenomenon but a transient that would elevate higher if the jet pulse were longer.

\footnotetext{
${ }^{1}$ Steady state refers to the flow when the Strouhal number is sufficiently large but before the drive time is over. It does not refer to the absence of changes between pulses.
} 

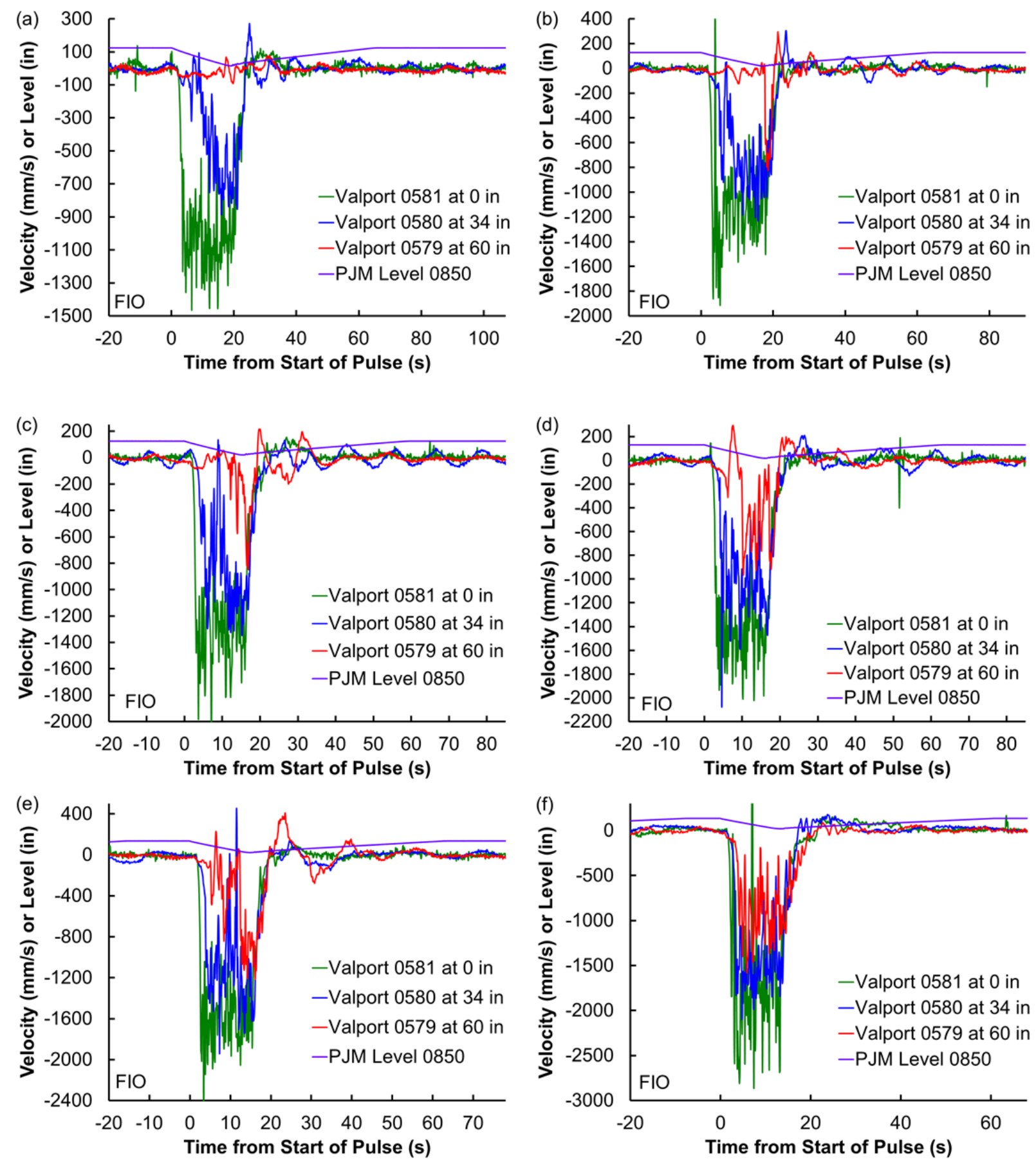

Figure 7.1. Central Upwell Velocity 0,34, and 60 inches Above the Seam for a Six-Part Simulant at Velocities of (a) $9.5 \mathrm{~m} / \mathrm{s}$ (time stamp 152733), (b) $10.5 \mathrm{~m} / \mathrm{s} \mathrm{(154932),} \mathrm{(c)} 11.5 \mathrm{~m} / \mathrm{s}$ (085206), (d) $12 \mathrm{~m} / \mathrm{s}$ (091401), (e) $13 \mathrm{~m} / \mathrm{s}$ (143502), and (f) $15 \mathrm{~m} / \mathrm{s}$ (102228) with $P V F=0.2$, $d_{o}=1.94$ inches, $f_{R}=0.6$. Surface flow observed for $U_{o} \geq 12 \mathrm{~m} / \mathrm{s}$.

A second insightful experiment was reported by Shekarriz et al. (1995b), who considered jets propagating in non-Newtonian fluids. Figure 7.2 shows the nozzle velocity divided by the centerline velocity plotted against scaled distance from the nozzle; see Eq. (2.2). They argue that for these jets, the 
leading vortex was essentially stationary, suggesting negligible jet celerity. Jets that fail to reach the surface in mixing vessels may exhibit a similar attenuation of jet front celerity. ${ }^{1}$

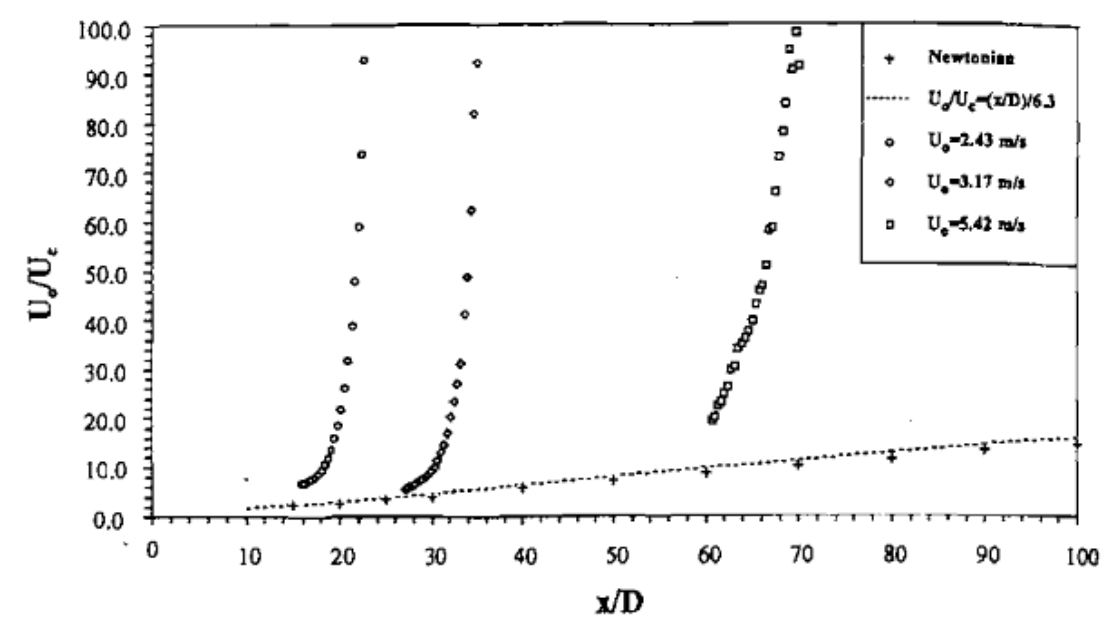

Figure 7.2. Nozzle Velocity Divided by Centerline Velocity Versus Distance from Nozzle Scaled on Nozzle Diameter from Shekarriz et al. (1995b). Original caption: "Fig. 9 Instantaneous center-line velocity within the terminating regions shown in Figs. 7 and 8." Reprinted with permission from ASME.

In any case, it is clear experimentally that some of the flows may not achieve the surface because their rate of propagation remains insufficient. Intuitively, the transit time for a jet from the seam to the surface may be estimated by recognizing $u_{m, c}=d z / d t$ and integrating Eq. (2.1) or its equivalents herein with an initial condition of $z=0$ at $t=0$. Using this logic, Meyer and Etchells (2007), Bamberger et al. (2005), Meyer et al. (2006), and Kurath et al. (2007) ${ }^{2}$ assert that

$$
t=\frac{d_{o}}{2 h_{c} U_{o}}\left(z / d_{o}\right)^{2}
$$

in the absence of a virtual origin. This equation may be rewritten in the form of Sangras and Faeth (1999):

$$
\frac{z}{d_{o}}=\sqrt{2 h_{c}}\left(\frac{t}{d_{o} / U_{o}}\right)^{1 / 2}
$$

from which alpha may be identified as the square root of $2 h_{c}$ and $n=1 / 2$. Therefore, factors that would influence $h_{c}$ also influence the celerity curves. Comparing this solution to the drive time may provide an estimate of whether the upwell would reach the surface in time. An expression for the transit time may be written for each region as

\footnotetext{
${ }^{1}$ Combining the stress balances of Cai et al. (2012), the celerity mushroom of Kuhn et al. (2013), and an elastic restoring force as given by Powell et al. (1997) may explain Shekarriz' s conundrum.

${ }^{2}$ Kurath et al. (2007) consider how this time scale with a virtual origin is affected by parallel or co-flow, which is not analyzed here but may affect these time scales at least marginally.
} 


$$
\begin{aligned}
& \frac{t_{\text {primary }}}{t_{D}}=\frac{d_{o}}{2 U_{o} t_{D}} \frac{\left(b / d_{o}\right)^{2}}{h_{j}} \\
& \frac{t_{\text {radial }}}{t_{D}}=\frac{d_{o}}{2 U_{o} t_{D}} \frac{\left(f_{R} D / d_{o}\right)^{2}}{4 h_{r}}
\end{aligned}
$$

and

$$
\frac{t_{\text {upwell }}}{t_{D}}=\frac{d_{o}}{2 U_{o} t_{D}} \frac{\left(z / d_{o}\right)^{2}}{h_{c}}
$$

scaled on the drive time. Adding the times to penetrate each region gives

$$
\frac{t}{t_{D}}=\frac{d_{o}}{2 U_{o} t_{D}}\left[\frac{\left(b / d_{o}\right)^{2}}{h_{j}}+\frac{\left(f_{R} D / d_{o}\right)^{2}}{4 h_{r}}+\frac{\left(z / d_{o}\right)^{2}}{h_{c}}\right]
$$

where $b$ is the standoff distance, $h_{j}$ is the coefficient at the nozzle $(\sim 6.3), h_{r}$ is the coefficient along the vessel bottom $(\sim 1.1)$, and $h_{c}$ is the coefficient of the upwell ( $\sim 1$ to 5$)$.

Table 7.1 shows that for the range of tests considered, $t / t_{D}$ is always less than 1 , implying that the jet always has sufficient time to reach the surface, even though Figure 7.1 shows that not to be the case. Because the particle-specific properties vanished in Section 4.2, this time-scale properly formulated is the only upwell time scale for the flow problem (turbulence itself has the only other independent time scale). Table 7.1 also shows that a strong majority of the transit time associated to achieve surface flow is

\begin{tabular}{|c|c|c|c|c|c|}
\hline & $t / t_{D}$ & $t_{\text {primary }} / t_{D}$ & $t_{\text {radial }} / t_{D}$ & $t_{\text {upwell }} / t_{D}$ & $t_{\text {upwell }} / t$ \\
\hline Eqs. 5.64-5.67, Short Case ${ }^{(a)}$ & $9.58 \cdot 10^{-2}$ & $5.30 \cdot 10^{-5}$ & $2.78 \cdot 10^{-2}$ & $6.79 \cdot 10^{-2}$ & $70.9 \%$ \\
\hline Eqs. 5.64-5.67, Long Case ${ }^{(a)}$ & 0.994 & $1.71 \cdot 10^{-3}$ & $7.56 \cdot 10^{-2}$ & 0.917 & $92.2 \%$ \\
\hline
\end{tabular}
associated with rising through the central upwell; therefore, this portion of the total time remains the focus for the remainder of this section.

Table 7.1. Penetration Times in the $8 \mathrm{ft}$ Vessel

(a) In the $8 \mathrm{ft}$ vessel tests with $d_{o}=1.94$ inches, $b=2.9-11.6$ inches, $f_{R}=0.6-0.7, D=92.5$ inches, $z / d_{o}=47.7-55.4$, $U_{o} t_{D}=82.5-165 \mathrm{~m}$ per Table 3.2.1 in run sheet 7. FIO.

A factor of three in uncertainty may be expected from a scaling analysis, but Eq. (7.2) misses essential factors that may affect its value. To determine what these factors may be, consider the following. In Section 4, the time-dependent momentum balance ${ }^{1}$ was shown in Eq. (4.16) (repeated below) to be

\footnotetext{
${ }^{1}$ The influence of bulk motion was not important at steady state, but its influence on transient jets remains unevaluated.
} 


$$
\begin{aligned}
\rho_{\infty} \frac{\partial u}{\partial t}+\rho_{\infty} v \frac{\partial u}{\partial r}+\rho_{\infty} u \frac{\partial u}{\partial z}= & -\frac{\partial \wp}{\partial z}-g \rho\left(\frac{\rho-\rho_{\infty}}{\rho_{\infty}}\right) \\
& +\frac{\tau_{o}}{r}+\mu_{o} \frac{1}{r} \frac{\partial}{\partial r}\left(r \frac{\partial u}{\partial r}\right)+\rho_{\infty} \frac{C^{2}}{r} \frac{\partial}{\partial r}\left[r z^{2}\left(\frac{\partial u}{\partial r}\right)^{2}\right]
\end{aligned}
$$

In the absence of governing pressure gradients (present but not governing), buoyancy, and yield and viscous stresses, this equation becomes

$$
\int_{0}^{\infty} \frac{\partial u}{\partial t} r d r+\int_{0}^{\infty} v \frac{\partial u}{\partial r} r d r+\int_{0}^{\infty} u \frac{\partial u}{\partial z} r d r=\int_{0}^{\infty} \frac{C^{2}}{r} \frac{\partial}{\partial r}\left[r z^{2}\left(\frac{\partial u}{\partial r}\right)^{2}\right] r d r
$$

Section 4.3 showed that the second two terms on the left may be combined and that the term on the right vanishes with integration over these limits. Then,

$$
\frac{\partial}{\partial t} \int_{0}^{\infty} u r d r+\frac{\partial}{\partial z} \int_{0}^{\infty} u^{2} r d r=0
$$

Substituting Eqs. (2.1), (2.2), and (2.4) finds

$$
\frac{\partial}{\partial t}\left(u_{m, c} \delta_{c}^{2} \int_{0}^{\infty} f_{c} \eta_{c} d \eta_{c}\right)+\frac{\partial}{\partial z}\left(u_{m, c}^{2} \delta_{c}^{2} \int_{0}^{\infty} f_{c}^{2} \eta_{c} d \eta_{c}\right)=0
$$

with

$$
\psi_{1,1} \equiv \int_{0}^{\infty} f_{c}\left(\eta_{c}\right) \eta_{c} d \eta_{c}
$$

and Eq. (3.6),

$$
\frac{\partial}{\partial t} u_{m, c}+\frac{\partial}{\partial z}\left(\frac{\psi_{2,1}}{\psi_{1,1}} u_{m, c}^{2}\right)=0
$$

This is a first order partial differential equation in two variables. Bajpai and Tirumkudulu (2008) recognized that first order differential equations may admit a jump or shock condition. ${ }^{1}$ The Rankine-Hugoniot jump condition (Eq. 7 in Bajpai and Tirumkudulu 2008) where the upstream velocity and flux are assumed to be zero becomes

\footnotetext{
${ }^{1}$ The derivation of Bajpai and Tirumkudulu (2008) contains a nonhomogeneity for which the Rankine-Hugoniot jump condition does not strictly hold.
} 


$$
\frac{d z}{d t}=\frac{\frac{\psi_{2,1}}{\psi_{1,1}} u_{m, c}{ }^{2}-0}{u_{m, c}-0}=\frac{\psi_{2,1}}{\psi_{1,1}} u_{m, c}
$$

(see Yarnovsky (2005) for a derivation of the Rankine-Hugoniot jump condition, which only strictly holds for homogeneous first order differential equations). Substitution of Eq. (2.1) leaves

$$
\frac{d z}{d t}=\frac{\psi_{2,1}}{\psi_{1,1}} \frac{h_{c} U_{o} d_{o}}{\left(z+z_{o}\right)}
$$

Integrating with an initial condition of $t=t_{o}$ at $z=z_{l}$, where $z_{l}$ is an elevation that corresponds to the lower limit of integration, gives

$$
\left(z+z_{o}\right)^{2}-\left(z_{l}+z_{o}\right)^{2}=2 \frac{\psi_{2,1}}{\psi_{1,1}} h_{c} U_{o} d_{o}\left(t-t_{o}\right)
$$

when a term of $d z_{o} / d t$ is added to the left-hand side of Eq. (7.14). Due to the nonlinearity of Eq. (7.15), either $t_{o}$ or $z_{l}$ may be selected by fiat but not both. Solving for $z$ gives

$$
\frac{z}{d_{o}}=\sqrt{\left(\frac{z_{l}}{d_{o}}+\frac{z_{o}}{d_{o}}\right)^{2}+2 \frac{\psi_{2,1}}{\psi_{1,1}} h_{c}\left(\frac{U_{o} t}{d_{o}}-\frac{U_{o} t_{o}}{d_{o}}\right)}-\frac{z_{o}}{d_{o}}
$$

This equation shows that the celerity equation is more intricate than Sangras and Faeth (1999) may have anticipated for tertiary jets. The virtual origin again plays an important role and the limits of integration matter. Even in their absence, these equations show that Eq. (7.3) misses the influence of the radial distributions on the jet flows as captured in the ratio of $\psi_{2,1} / \psi_{1,1}$.

Figure 7.3 presents celerity curves from both historical data and recent $8 \mathrm{ft}$ testing. Figure $7.3 \mathrm{a}$ and $\mathrm{b}$ show the temporal evolution of the jet front for water in both the 12.75 and $8 \mathrm{ft}$ vessels. Comparing Figure 7.3a and $\mathrm{b}$ shows that either increasing the number of PJMs from four to six or increasing the velocity from $<9$ to $12 \mathrm{~m} / \mathrm{s}$ substantially shortens the rise time, even though it takes longer for the radial jet that feeds the central upwell to reach the vessel center in the $8 \mathrm{ft}$ vessel. Either approximately doubles the momentum feeding the upwell.

In the presence of solids, Figure $7.3 \mathrm{c}$ and e suggest that there is a small but perceptible increase in the celerity time with increased solids loadings when the nozzle velocity is held approximately constant at $\sim 8$ to $9 \mathrm{~m} / \mathrm{s}$ in the $12.75 \mathrm{ft}$ vessel or $15 \mathrm{~m} / \mathrm{s}$ in the $8 \mathrm{ft}$ vessel. Whether the various solids loadings would cluster together at other nozzle velocities has not been explored experimentally, but is important because nozzle velocities in substantial excess of $12 \mathrm{~m} / \mathrm{s}$ are not anticipated in the $16 \mathrm{ft}$ vessel. However, Figure $7.3 \mathrm{~d}$ shows a strong dependence on the nozzle velocity. As the nozzle velocity drops from $15 \mathrm{~m} / \mathrm{s}$ down to $10.5 \mathrm{~m} / \mathrm{s}$, the upwell time more than doubles. This change is important. For a nozzle diameter of 1.94 inches in the 92.5 inch test vessel $(\sim 8 \mathrm{ft})$, the product of $t_{D} U_{o} / d_{o}=1680,2520$, and 3350 for 
$\mathrm{PVF}=0.10,0.15$, and 0.20 , respectively. This explains why the six-part simulant with a PVF of 0.20 in the $8 \mathrm{ft}$ vessel may be successful at approximately $12 \mathrm{~m} / \mathrm{s}$ but not at $11.5 \mathrm{~m} / \mathrm{s}$.

That a nozzle velocity dependence may arise in the experimental data when plotted against $t U_{o} / d_{o}$ is not obvious from the functional form of Eq. (7.16). Such an implicit dependence may arise through any of the parameters in the equation. For example, Figure $7.3 \mathrm{f}$ displays $\psi_{21} / \psi_{11}$ versus the nozzle velocity. Remarkably, as the nozzle velocity increases from $11 \mathrm{~m} / \mathrm{s}$, the value of this ratio increases, commensurate with significant entrainment of peripheral slurry, which in turn appears to concentrate the particles with their momentum closer to the centerline of the jet. Conversely, as the value of this ratio decreases, the radial distribution flattens out, as may be expected of a velocity profile approaching a cloud height barrier, even a transient one.

In the anticipated $16 \mathrm{ft}$ vessel, the nozzle diameter approximately doubles relative to the $8 \mathrm{ft}$ vessel, but the drive time also doubles, suggesting that the dimensionless group $t_{D} U_{o} / d_{o}$ will remain approximately (though not precisely) the same. This would suggest that if the jet remains free, the $16 \mathrm{ft}$ vessel will perform approximately as well as the $8 \mathrm{ft}$ vessel. However, Eq. (7.16) was derived asserting that the buoyancy and yield stresses remain negligible. The former may or may not influence the jet celerity curves and therefore the scaling between vessel sizes. Confidence requires evaluation of the influence of $\rho_{\max } / \rho_{\infty}$ and $z_{o} / d_{o}$ as a function of geometric scale similar to the cloud height arguments in Section 5. 

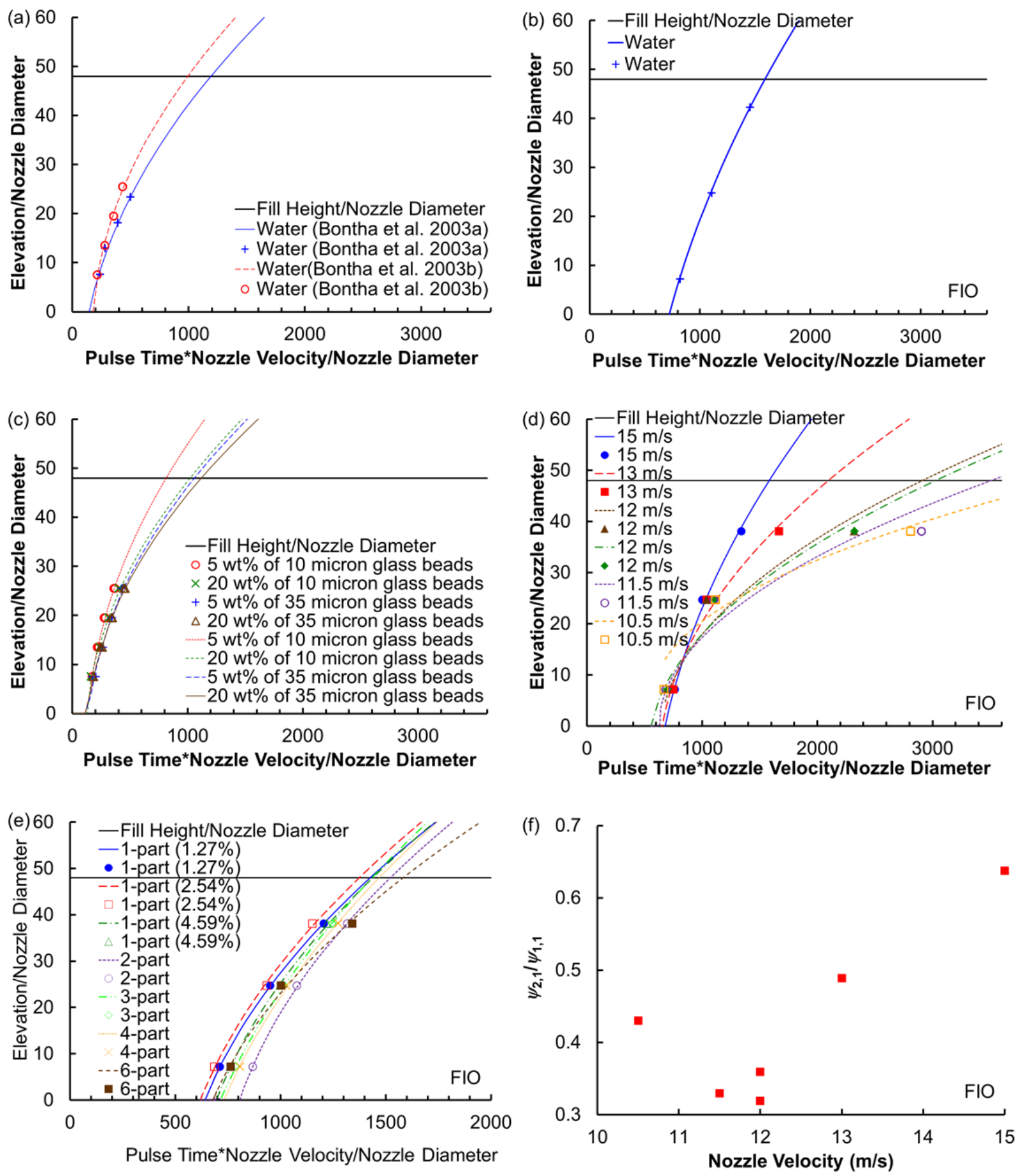

Figure 7.3. Elevation Scaled on Nozzle Diameter Versus Time Scaled on the Jet Time (ratio of the nozzle diameter to the nozzle velocity) for (a) Water in the $12.75 \mathrm{ft}$ Vessel and (b) Water in the $8 \mathrm{ft}$ Vessel at $12 \mathrm{~m} / \mathrm{s}$, (c) for Glass Particles (10 and $35 \mu \mathrm{m}$ Glass Beads at 5 and $20 \mathrm{wt} \%$ ) in the $12.75 \mathrm{ft}$ Vessel, (d) for the Six-Part Simulant in the $8 \mathrm{ft}$ Vessel at Various Velocities, and (e) the Multipart Simulants in the $8 \mathrm{ft}$ Vessel at $15 \mathrm{~m} / \mathrm{s}$, (f) the $\psi_{2,1} / \psi_{1,1}$ Ratio versus Nozzle Velocity for the Multipart Simulants in the $8 \mathrm{ft}$ Vessel 
Inversely, Eq. (7.15) may be solved for $t_{\text {upwell }}=t-t_{o}$ as

$$
\frac{t_{\text {upwell }}}{t_{D}}=\frac{d_{o}}{2 U_{o} t_{D}} \frac{\psi_{1,1}}{\psi_{2,1}} \frac{\left[\left(\frac{z}{d_{o}}+\frac{z_{o}}{d_{o}}\right)^{2}-\left(\frac{z_{l}}{d_{o}}+\frac{z_{o}}{d_{o}}\right)^{2}\right]}{h_{c}}
$$

The presence of a nonzero virtual origin increases the upwell time. Perhaps more insightfully,

Table 3.1 finds $\psi_{1,1}$ to be greater than $\psi_{2,1}$ by approximately a factor of 1.33 to 3.00, which immediately implies that the upwell takes longer because of the radial distribution of velocities neglected previously. That the actual time may be approximately double that of the jet time is consistent with the arguments of Kuhn et al. (2013, Eqs. C.38-C.39), who then use that information to predict the radial spread of the leading vortex relative to the width of the jet itself. They also indicate that the radial head provides an additional impediment to momentum that may affect the value of $h_{c}$ during the transient stages of jet propagation. To determine the overall time to surface flow, a similar analysis is required on the radial jet. This would be a straightforward analysis because the same governing equations apply with only a change in boundary conditions. In the interest of schedule, an overall expression for the time to surface flow is not included, preventing quantitative comparison to experimental data.

Table 7.2 compares the upwell-time-to-drive-time ratio for the tests in the $8 \mathrm{ft}$ vessel Figure 7.4 shows the same information graphically. When $t_{\text {upwell }} / t_{D}$ remains small surface flow is achieved because the jet reaches the surface before the drive time ends. In contrast, when the time ratio is large, the upwell takes longer to reach the surface than the drive time allows. Some of the cases for which there is no surface flow may have ratios that are below one, but in nearly each of these cases (except the KB slurry and the six-part simulant) the range spans unity and this ratio does not include the time it takes the radial jets to travel from PJM nozzle to vessel centerline.

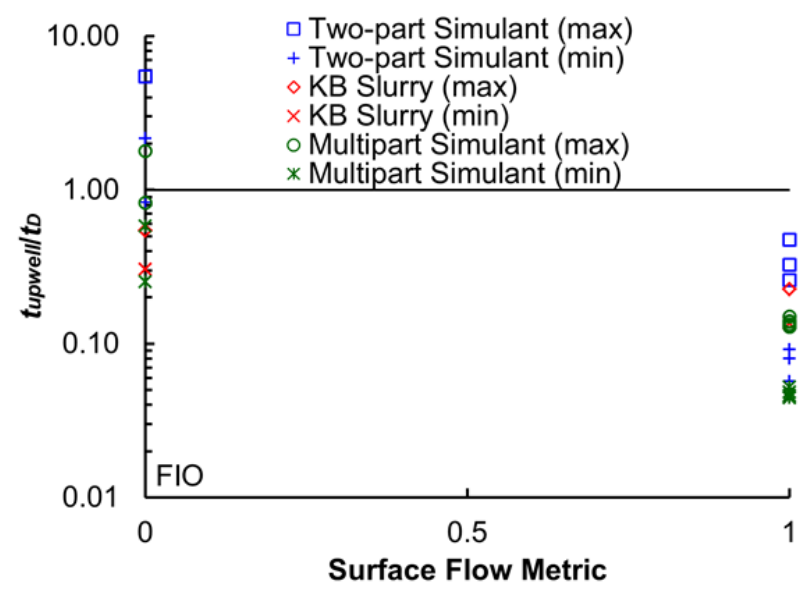

Figure 7.4. The Ratio of the Upwell Time to the Drive Time Differentiated on the Surface Flow Metric of Table 2.3. When this time ratio is small, surface flow should be achieved, and when it is large, the upwell does not reach the surface before the jet turns off. 
Table 7.2. Upwell Times in the $8 \mathrm{ft}$ Vessel $^{(a)}$

\begin{tabular}{|c|c|c|c|}
\hline Reference & Constituents & Surface Flow & $t_{\text {upwell }} / t_{D}$ \\
\hline Commissioning & Water & NR & $0.091-0.26$ \\
\hline Run sheet 1 & Glass powder, $\mathrm{Al}_{2} \mathrm{O}_{3}$ & Y & $0.057-0.33$ \\
\hline Run sheet 1 & Glass powder, $\mathrm{Al}_{2} \mathrm{O}_{3}$ & $\mathrm{~N}$ & $2.2-14$ \\
\hline Run sheet 2 & Glass powder, $\mathrm{Al}_{2} \mathrm{O}_{3}$ & Y & $0.080-0.47$ \\
\hline Run sheet 2 & Glass powder, $\mathrm{Al}_{2} \mathrm{O}_{3}$ & $\mathrm{~N}$ & $0.83-5.5$ \\
\hline Run sheet 3 & KB slurry, $U_{o} \sim 12 \mathrm{~m} / \mathrm{s}$ & Y & $0.14-0.23$ \\
\hline Run sheet 3 & KB slurry, $U_{o} \sim 8 \mathrm{~m} / \mathrm{s}$ or A2 firing & $\mathrm{N}$ & $0.30-0.54$ \\
\hline Run sheet 7 & Glass powder, $1.27 \mathrm{wt} \%, U_{o}=15 \mathrm{~m} / \mathrm{s}$ & Y & $0.046-0.13$ \\
\hline Run sheet 7 & Glass powder, $2.54 \mathrm{wt} \%, U_{o}=15 \mathrm{~m} / \mathrm{s}$ & Y & $0.045-0.13$ \\
\hline Run sheet 7 & Glass powder, $4.59 \mathrm{wt} \%, U_{o}=15 \mathrm{~m} / \mathrm{s}$ & Y & $0.045-0.13$ \\
\hline Run sheet 7 & Glass powder, $\mathrm{W},{ }^{(\mathrm{b})} U_{o}=15 \mathrm{~m} / \mathrm{s}$ & Y & $0.048-0.14$ \\
\hline Run sheet 7 & Glass powder, $\mathrm{W}^{(\mathrm{b})} \mathrm{SiC}, U_{0} \geq 12.0 \mathrm{~m} / \mathrm{s}$ & $\mathrm{Y}$ & $0.048-0.14$ \\
\hline Run sheet 7 & Glass powder, $\mathrm{W}^{(\mathrm{b})} \mathrm{SiC}, U_{o} \leq 9.5 \mathrm{~m} / \mathrm{s}$ & $\mathrm{N}$ & $0.58-1.8$ \\
\hline Run sheet 7 & Glass powder, $\mathrm{W},{ }^{(\mathrm{b})} \mathrm{SiC}$, beads, $U_{o}=15.0 \mathrm{~m} / \mathrm{s}$ & Y & $0.0468-0.13$ \\
\hline Run sheet 7 & Glass powder, $\mathrm{W}^{(\mathrm{b})} \mathrm{SiC}$, beads, $\mathrm{Al}_{2} \mathrm{O}_{3}$, sand, $U_{o} \geq 12.0 \mathrm{~m} / \mathrm{s}$ & Y & $0.052-0.15$ \\
\hline Run sheet 7 & Glass powder, $\mathrm{W}^{(\mathrm{b})} \mathrm{SiC}$, beads, $\mathrm{Al}_{2} \mathrm{O}_{3}$, sand, $U_{o} \leq 11.5 \mathrm{~m} / \mathrm{s}$ & $\mathrm{N}$ & $0.25-0.83$ \\
\hline
\end{tabular}

(a) Based on $h_{c}$ and $z_{o} / d_{o}$ in Table 2.4, $z / d_{o}=48-55$ for $d_{o}=1.94$ inches, and $\psi_{2,1} / \psi_{1,1}$ from Table 3.1 where available for the specific test or the minimum and maximum generally of $\psi_{2,1} / \psi_{1,1}$ from the Table 3.1 otherwise. FIO.

(b) $\mathrm{W}$ denotes the dense tungsten component, tungsten carbide with a density of $9.6 \mathrm{~g} / \mathrm{cm}^{3}$.

With modifications and comparison to experiment, analysis of jet celerity may become a compelling tool to evaluate the scalability between vessel sizes from first principles. For example, the buoyancy and yield stresses may be included in this analysis. After radial integration, this results in first order differential equations as above. However, these differential equations are nonhomogeneous so that the Rankine-Hugoniot jump condition does not strictly apply. The method of characteristics may be used to identify the jump criteria in this case as given in Yarnovsky (2005). Lin (2000) considers quasilinear hyperbolic systems and uses the method of vanishing viscosity to construct shock solutions that provide insight into compressive and fan shocks that arise due to the nonhomogeneity and traveling waves associated with the shock. Russel et al. (1989, Chapter 12) consider similar discontinuities that arise in the context of settling. Jenny and Muller (1998) show that the nonhomogeneities (here buoyancy or yield stresses) smooth the jet front as observed experimentally so that direct simple solutions may be obtained, uncertainty reduced, and confidence in scaling increased.

In conclusion, this section evaluates the celerity (i.e., the rate at which the jet front rises) of the central upwell. The analysis is confined to a free jet and reasonably discriminates between conditions that induce surface flow during the drive cycle and those that do not. The analysis is incomplete because the influence of decelerating yield stresses and negative buoyancy, if any, has not yet been fully evaluated. 


\subsection{Wall Upwell Velocity Profiles}

The jet physics along the wall remains different from the physics along the vessel centerline and should not be conflated. To put this assertion into perspective, here the velocity profile along the wall is derived parallel to the vertical velocity profiles discussed in Section 3. Consider an annular control volume, the top of which is defined on the inside (radially) by a hexagon with vertices at the centerlines of PJM nozzles and along the outside by the vessel wall. This is the portion of the vessel head not included in the control volume of Section 3.1. The portion of the momentum from the nozzles entering this control volume is $N\left(1-f_{N}\right)$, where $f_{N}$ was defined in Section 3.1 and represents the fraction of jet flow moving toward the centerline of the vessel. The jet momentum leaving the nozzles, which is the jet momentum that drives the wall upwell in the absence of friction losses from shear along the vessel bottom, is then

$$
M_{o}=\frac{\pi}{4} N\left(1-f_{N}\right) \rho_{o} U_{o}^{2} d_{o}^{2}
$$

Integrating the steady-state Navier-Stokes equations with time-averaged Reynolds stresses dominating viscous stresses gives Eq. 10-12 of Rajaratnam (1976), written here as

$$
\frac{d}{d z} \int_{0}^{\infty} \rho u_{w}{ }^{2} d y=-\tau_{w}
$$

where $\tau_{w}$ is the turbulent shear stress at the wall and $u_{w}(y, z)$ is the vertical velocity along the wall with vertical coordinate $z$ and horizontal coordinate $y$ as measured from the wall. This expression uses the Derjaguin approximation, which asserts that the jet is thin relative to the circumference of the vessel, such that Cartesian coordinates may be used to describe the jet, much like Cartesian coordinates may be used on the surface of the Earth even though it has finite curvature. Integrating both sides and recognizing $\tau_{w}$ to first order approximation to be independent of $z$ gives

$$
\int_{0}^{\infty} \rho u_{w}{ }^{2} d y=-\tau_{w} z+\frac{M_{o}}{\pi D}
$$

where the final term is an integration constant that must represent the jet momentum at $z=0$ per unit length along the vessel circumference, $\pi D$, to preserve dimensionally consistency. The velocity along the wall is self-similar ${ }^{1}$ in an otherwise quiescent media so that

$$
u_{w}(z, y)=u_{m, w} f_{w}\left(\eta_{w}\right)
$$

where $u_{m, w}$ is the maximum axial velocity of the jet parallel to the wall that depends only on $z, f_{w}$ is a functional form defined by this equation, and

$$
\eta_{w}=y /\left[\beta_{w}\left(z+z_{o}\right)\right]=y / \delta_{w}
$$

\footnotetext{
${ }^{1}$ Strictly true in quiescent media (Rajaratnam 1976, Chapter 10), and often true where there is flow parallel to the jet (Rajaratnam 1976, Chapter 12).
} 
where $\beta_{w}$ characterizes the radial spread of the wall upwell and $z_{o}$ is the virtual origin for the wall jet (Kuhn et al. 2013; Rajaratnam 1976). The shear stress at the wall can be described in terms of the skin coefficient of friction with $c_{f}=2 \tau_{w} / \rho_{o} u_{m, w}{ }^{2}$ (Rajaratnam 1976; Beltaos and Rajaratnam 1977; Meacham et al. 2012). Substituting gives

$$
\rho u_{m, w}{ }^{2} \int_{0}^{\infty} f_{w}{ }^{2} d y=-\frac{c_{f}}{2} \rho_{o} u_{m, w}{ }^{2} z+\frac{M_{o}}{\pi D}
$$

Eq. 1.27 of Verhoff (1963) gives the functional form of $f_{w}$ as

$$
f_{w}\left(\eta_{w}\right)=1.4794 \eta_{w}{ }^{1 / 7}\left(1-\operatorname{Erf}\left(0.67753 \eta_{w}\right)\right)
$$

The integral then becomes a constant,

$$
\psi_{2,0}=\int_{0}^{\infty} f_{w}{ }^{2} d \eta_{w}=0.75429
$$

retaining Verhoff's significant figures. Solving for $u_{m, w}$ gives

$$
u_{m, w}{ }^{2}=\frac{M_{o}}{\pi D\left(\rho \beta_{w}\left(z+z_{o}\right) \psi_{2,0}+\frac{c_{f}}{2} \rho_{o} z\right)}
$$

Substitution of Eq. (8.1) finds

$$
u_{m, w}=c_{j} \frac{U_{o} d_{o}}{\sqrt{D\left(z+z_{o}\right)}} \sqrt{\frac{N\left(1-f_{N}\right)}{4 \frac{\rho}{\rho_{o}} \beta_{w} \psi_{2,0}+\frac{2 c_{f} z}{z+z_{o}}}}
$$

where $c_{j}$ represents an additional jet constant (if needed to fit data). Scaling the local velocity on the nozzle velocity and the elevation on the nozzle diameter gives

$$
\frac{u_{m, w}}{U_{o}}=\frac{c_{j}}{\sqrt{\frac{D}{d_{o}} \frac{z+z_{o}}{d_{o}}}} \sqrt{\frac{N\left(1-f_{N}\right)}{4 \frac{\rho}{\rho_{o}} \beta_{w} \psi_{2,0}+\frac{2 c_{f} z}{z+z_{o}}}}
$$

This would seem to suggest that the value inside the square root depends significantly on the elevation. However, in absence of a virtual origin, the factor of $z /\left(z+z_{o}\right)$ vanishes, leaving $2 c_{f}$ as the second term in the denominator, which is small. Where $z_{o}>0$, the value of this term decreases even further. Eq. (3.19) provides an expression for the skin friction coefficient. With $\rho_{o}=1000-1200 \mathrm{~kg} / \mathrm{m}^{3}, U_{o}=3.7-15 \mathrm{~m} / \mathrm{s}$, $d_{o}=1.94-2.5$ inches, and $\mu_{o}=1.0-30 \mathrm{mPa} s, c_{f}=0.0060-0.017$ from Eq. (3.19). For comparison, $\beta_{w}$ is similar (Rajaratnam (1976) in Eq. 10-36 suggests $\beta_{w}=0.068$, though this value may vary significantly with solids 
loadings, see Table 2.1), leaving the first term in the denominator of Eq. (8.11) approximately an order of magnitude larger than the second term.

For comparison, the velocity along the centerline as given in Eq. (3.8) (repeated below) is

$$
\frac{u_{m, c}}{U_{o}}=\frac{c_{j}}{\beta_{c}} \sqrt{\frac{N f_{N}}{8 \psi_{2,1}} \frac{\rho_{o}}{\rho}} \frac{1}{z / d_{o}+z_{o} / d_{o}}
$$

Although both expressions do not have an intercept other than the virtual origin, the two expressions differ in key ways. First, the velocity along the wall depends approximately on the square root of the elevation. This dependence was anticipated without evaluation by Meyer and Etchells (2007) and decreases the rate at which velocity decays with elevation. Second, unlike the central upwell velocity, the tank diameter plays an integral role in the velocity along the wall because the jet's proximity to the vessel perimeter must influence jet flow. It may, therefore, not be surprising that statistical analyses (e.g., cloud height, Meyer et al. 2012) would include tank diameter and possibly find a dependence, though statistical analyses may conflate or block covarying factors (Wells et al. 2015). As in Section 3.2, shear forces along the vessel bottom may further strengthen such a relationship. However, because the tank diameter and elevation both scale on nozzle diameter and $c_{f}$ is small, Eq. (8.11) would suggest that geometric scaling will preserve the scaled velocity. Third, the distinction between central and wall upwells has important implications for cloud and "cavern" heights. Cloud heights have been studied experimentally (Enderlin et al. 2003; Meyer et al. 2012), statistically (Meyer et al. 2012; Kuhn et al. 2013), and theoretically for two impinging PJM flows (Kuhn et al. 2013). Discussion of cloud height, however, often conflates measurements that are taken along the wall with those that are made along the vessel centerline. Meyer et al. (2012) indicate that they measured cloud height at the center of the vessel, though solids may also have been elevated in the annulus between the PJM rings. Enderlin et al. (2003) measured the cloud height along the wall of their vessel. The recent measurements of cloud height in the $8 \mathrm{ft}$ test vessel have been measured using cameras and tape measures at the vessel wall (Bontha et al. 2015), though cloud height estimates for the final run sheet 7 may or may not be based on the vessel center or wall. Therefore, comparison of Eqs. (8.11) and (3.8) shows that the functional forms are distinct and not interchangeable, with the direct implication that the functional forms of cloud and "cavern" heights formed along the wall must be distinct from those formed along the vessel center.

Eq. (8.11) may be rewritten as

$$
\frac{u_{m, w}}{U_{o}}=\frac{h_{w}}{\sqrt{\frac{z+z_{o}}{d_{o}}}}
$$

with

$$
h_{w} \equiv \frac{c_{j}}{\sqrt{\frac{D}{d_{o}}}} \sqrt{\frac{N\left(1-f_{N}\right)}{4 \frac{\rho}{\rho_{o}} \beta_{w} \psi_{2,0}}}
$$

when $c_{f}$ remains small. This form of Eq. (8.12) motivates fitting experimental data with 


$$
\left(\frac{U_{o}}{u_{m, w}}\right)^{2}=\frac{1}{h_{w}{ }^{2}} \frac{z}{d_{o}}+\frac{1}{h_{w}{ }^{2}} \frac{z_{o}}{d_{o}}
$$

similar to what was done with the central upwell. Review of the data from the $8 \mathrm{ft}$ vessel considered only a limited subset of experimental data to support this equation. Therefore, the estimates below should only be considered a first approximation or estimate of wall jet properties. Further uncertainty arises in that the location of the maximum velocity should not remain a constant distance radially inward from the vessel wall, but should increase with elevation due to Eq. (8.7), whereas the velocity probes did not vary in distance away from the wall with elevation. Nevertheless, the available data are insightful.

Figure 8.1 is characteristic of the velocity data that showed signs of turbulence at the middle probe. The lowest probe at the seam shows strong and significant velocities $4.1 \pm 0.4 \mathrm{~s}(1 \sigma)$ after the pulse is registered as a PJM level drop. ${ }^{1}$ The jet arrives at the second probe 34 inches above the seam $7.7 \pm 1.3 \mathrm{~s}$ $(1 \sigma)$ after the pulse begins. (Note its arrival is slower than at lower nozzle velocities in Figures 2.8 and 2.10.) What is remarkable is that the top probe first registers a bulk flow, but before upwell associated turbulence may set in, the direction of the velocity reverses and shows signs of turbulence. About a second later, the velocity at the middle probe falls to an average near $0 \mathrm{~mm} / \mathrm{s}$. This suggests turbulent flow from the central upwell reaches the upper velocity probe first and that the velocity at the middle probe, while turbulent, is affected by both upward flow along the wall and downward flow from the upper surface. In the analysis that follows, only the portion of the velocity profiles prior to the arrival of the downward flow is used to estimate the jet coefficients.

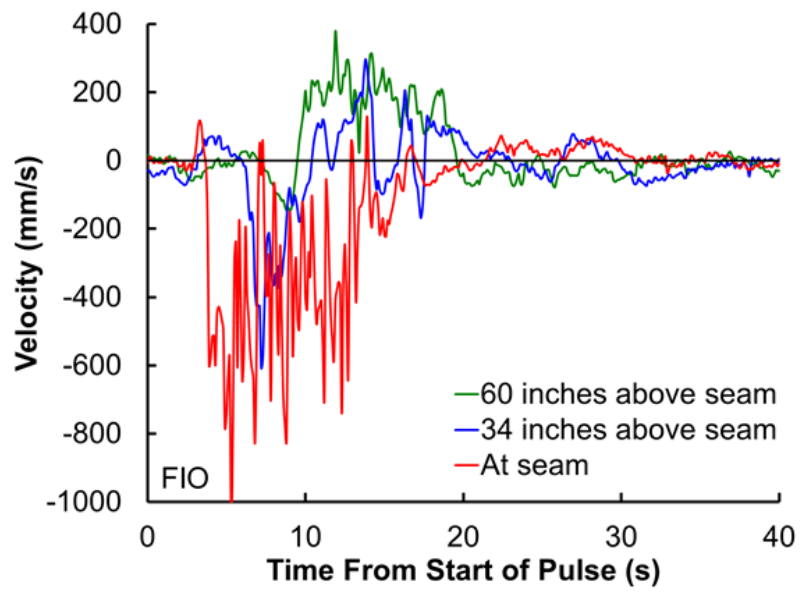

Figure 8.1. Velocity Profiles Along the Wall 0 inches (red, Valeport 0578), 34 inches (blue, Valeport 0577), and 60 inches (green, Valeport 0576) Above the Seam for Test 7-8.6.2 (time stamp 103104) for the Three-Part Simulant with $U_{o}=15 \mathrm{~m} / \mathrm{s}, d_{o}=1.94$ inches, PVF $=0.2$, and $\mathrm{S}$ Firing. Negative probe velocities represent vertically upward flow.

For the three-part and six-part simulants, $h_{w}=0.088 \pm 0.042(1 \sigma)$ and $h_{w}=0.110 \pm 0.055(1 \sigma)$, respectively, indicating that the jet coefficients are not of distinct populations for these two simulants. These values are markedly smaller than those for $h_{c}$. By comparison, Eq. (8.13) predicts $h_{w}=0.64$ (for

\footnotetext{
${ }^{1}$ Bontha et al. (2015) indicate a 2.25 s delay in registering velocities relative to registering information from other sensors.
} 
$c_{j}=1$ ) for $N=6, f_{N}=1 / 3, \rho / \rho_{o}=1, \beta_{w}=0.068$ (Rajaratnam 1976, Eq. 10-36 for no solids), $d_{o}=1.94$ inches, and $D=92.5$ inches, leaving the ratio of $h_{w}$ from experiment to $h_{w}$ for a free wall jet as $c_{j} \sim 1 / 6$. This value of $c_{j}$ is not particularly surprising because it is within a factor of $\sim 2$ of the estimates in Table 3.3 without the inclusion of negative buoyancy effects (see the velocity profile prediction of Section 5.2).

As a purely empirical discovery, Figure 8.2 compares the $z_{o} / d_{o}$ to $h_{w}$ and finds an approximate linear trend in the profile. This is similar to the linear trend found in Figure 3.1 for $z_{o} / d_{o}$ versus $h_{c}$ for the central upwell.

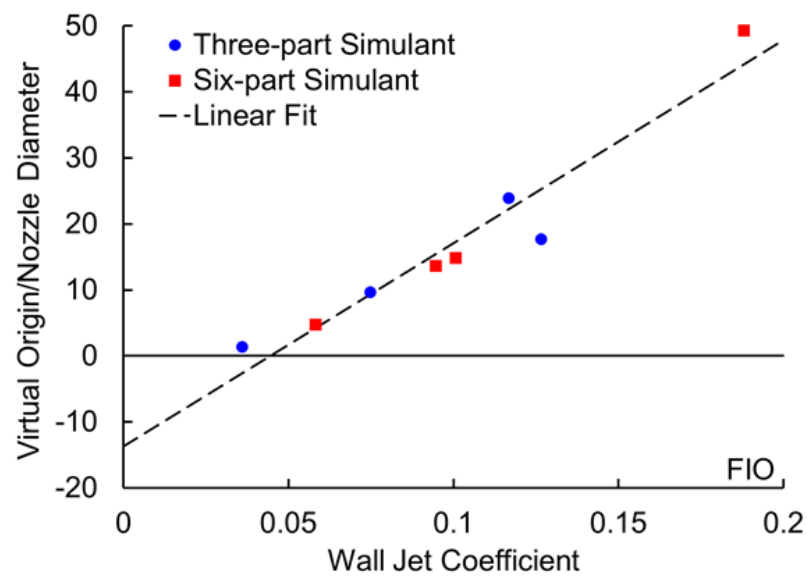

Figure 8.2. Depth of the Virtual Origin below the Seam/Tangent Scaled on Nozzle Diameter, $z_{o} / d_{o}$, Versus the Wall Jet Coefficient, $h_{w}$, for the Three-Part and Six-Part Simulants

In conclusion, this section considers upward directed wall jets. Based on a limited data set, the jet coefficient for the wall jet has been shown to be an order of magnitude smaller than the jet coefficient for the central upwell. The depth of the virtual origin scaled on the nozzle diameter appears to depend approximately linearly on the jet coefficient. Downward flows along the vessel wall oppose the upward jets limiting the rise of the upward jets. The analysis should be repeated on a larger data set where better statistics are desired. Section 9 discusses the implications of these profiles on the fraction of the vessel mixed. 



\subsection{Implications for Blending}

The development above has important implications for blending generally within the vessel in addition to that discussed above. This section specifically addresses the proportion of the vessel that is blended. Analysis builds on the profiles for the central and wall upwells developed previously. This section then explains why the volume mixed may exceed that defined by the jets themselves and why measurements to date remain insufficient to fully quantify this volume.

\subsection{Fraction of Vessel Blended}

The development of expressions for both the central and wall upwells permits estimation of the fraction of the vessel blended for free jets. This analysis considers the narrow question of what fraction of the vessel is mixed by the upwells at the moment the central upwell reaches the upper surface and then what volumetric flow rate is processed by the conical jet thereafter. The analysis is limited to a single pulse, for a full analysis of vessel mixing must incorporate the suction phase, downwell along the vessel sidewalls, and flows peripheral to the central upwell.

Where surface flow is achieved for free jets, this analysis begins with the assertion that fluid below the knuckle, tangent, or seam is well mixed. Though evaluation of bottom motion remains outside the present scope (see Beltaos 1975; Beltaos and Rajaratnam 1977), the experimental data files find that turbulent flow is significant and vigorous at the seam in the $8 \mathrm{ft}$ vessel, and upwell only becomes an influential consideration if bottom motion has been achieved. ${ }^{1}$ For a spherical head, as in the $8 \mathrm{ft}$ vessel, there is no knuckle so the volume is just that of a partial sphere, given as

$$
V_{\text {head }}=\frac{\pi}{6} h_{\text {head }}\left(3 r_{\text {head }}^{2}+h_{\text {head }}^{2}\right)
$$

where $r_{\text {head }}$ is the radius at the base of the head or cap and $h_{\text {head }}$ is the height of the cap or head. For a 2:1 elliptical head, Fink and Peterson (2015) give

$$
V_{\text {head }}=\pi D^{3} / 24
$$

Above the vessel bottom, the center and wall upwells may define volumes of blending.

The volume of the central upwell from the seam, knuckle, or tangent to the surface may be estimated as a right truncated cone given by a volume of revolution, $V_{\text {central }}$, as

$$
V_{\text {central }}=\pi \int_{z_{l}}^{z_{u}} r_{\text {cone }}{ }^{2} d z
$$

\footnotetext{
${ }^{1}$ Conversely, though not necessarily a physical inevitability (e.g., very thick particle beds), review of the last summary tables in Bontha et al. (2015) finds that surface flow is achieved only when bottom motion has also been achieved. Ascertainment of surface flow by the central upwell may serve, with further validation outside the scope of this document, as a conservative indicator of bottom motion in non-transparent vessels.
} 
where $z_{u}$ is the level of the upper fluid surface and $z_{l}$ is the lower limit of integration, here asserted to be the seam, tangent, or knuckle of the vessel. The radius of this cone, $r_{\text {cone }}$, may be determined by rearranging Eq. (2.4) as

$$
r_{\text {cone }}(z)=\eta_{c}^{99}\left[\beta_{c}\left(z+z_{o}\right)\right]
$$

where $\eta_{c}{ }^{99}$ represents the scaled position that encompasses $99 \%$ of the jet flow defined by $f_{c}\left(\eta_{c}{ }^{99}\right)=0.01$. Table 3.1 summarizes the values of $\eta_{c}^{99}$ for both theoretical curves and fits of experimental data to the normalized Fermi-Dirac function, and values of $\beta_{c}$ are given in Table 2.1. Substituting gives

$$
V_{\text {central }}=\frac{\pi}{3}\left(\eta_{c}^{99} \beta_{c}\right)^{2}\left[\left(z_{u}+z_{o}\right)^{3}-\left(z_{l}+z_{o}\right)^{3}\right]
$$

from which the volume of central upwell after passage of the leading vortex at the upper surface may be estimated. Alternatively, if the central upwell is a right cylindrical tube, then

$$
V_{\text {central }}=\pi R_{\text {jet }}^{2}\left(z_{u}-z_{l}\right)
$$

Similarly, the volume of the wall upwell from the seam, knuckle, or tangent to the surface is the volume of a right cylinder less a truncated cone. The radius of this cone is defined through the distance from the vessel wall to edge of the jet

$$
y_{\text {cone }}(z)=\eta_{w}^{99}\left[\beta_{w}\left(z+z_{o}\right)\right]
$$

found by rearranging Eq. (8.5), where $\eta_{w}{ }^{99}$ represents the scaled position that encompasses $99 \%$ of the jet flow defined by $f_{w}\left(\eta_{w}{ }^{99}\right)=0.01$. From the Verhoff (1963) profile (Eq. (8.7)), $\eta_{w}{ }^{99}=2.8786$, again retaining his significant figures. Rajaratnam (1976) in Eq. 10-36 suggests $\beta_{w}=0.068$, but the value of $\beta_{w}$ remains unmeasured in the presence of significant solids loadings and the uncertainty in this number remains unestimated. Then, the volume of the wall jet may be determined as a volume of revolution with a cone defined by $D / 2-y_{\text {cone }}$ subtracted from a right cylinder of diameter $D$ as

$$
V_{\text {wall }}=\frac{\pi}{4} D^{2}\left(z_{t}-z_{l}\right)-\pi \int_{z_{l}}^{z_{t}}\left(\frac{D}{2}-y_{\text {cone }}\right)^{2} d z
$$

where $z_{t}$ is the highest elevation that the wall jet achieves. Please note that $z_{t}$ may be time dependent. Substituting, integrating, and simplifying gives

$$
V_{\text {wall }}=\frac{1}{2} \pi D \eta_{w}{ }^{99} \beta_{w}\left[\left(z_{t}+z_{o}\right)^{2}-\left(z_{o}\right)^{2}\right]-\frac{\pi}{3}\left(\eta_{w}{ }^{99} \beta_{w}\right)^{2}\left[\left(z_{t}+z_{o}\right)^{3}-z_{o}{ }^{3}\right]
$$

where the second term remains smaller than the first and $z_{l}$ may be set to zero by establishment of a datum at the tangent, seam or knuckle.

The total volume is then

$$
V_{\text {total }}=V_{\text {head }}+V_{\text {central }}+V_{\text {wall }}
$$


After the flow has reached the fluid surface, additional volume often circulates through the jets. The fluid-entrained flow through the jet may be estimated from the volumetric flow rate at the liquid-free surface. The volumetric flow rate increases with elevation as the jet entrains fluid as it rises. The volumetric flow rate at the fluid surface (hypothetic since the jet turns there), therefore, represents all the flow into the central or wall upwells. For the central upwell, the volumetric flow $Q_{\text {central }}$ is

$$
Q_{\text {central }}=2 \pi \int_{0}^{\infty} u r d r
$$

Substituting Eqs. (2.1), (2.3), and (2.4) leaves

$$
Q_{\text {central }}=2 \pi \beta_{c}^{2} h_{c} U_{o} d_{o} \psi_{1,1}\left(z_{u}+z_{o}\right)
$$

where $\psi_{1,1}$ is a numerical constant defined in Eq. (7.11) and given in Table 3.1.

Under the Derjaguin approximation for a wall jet,

$$
Q_{\text {wall }}=\pi D \int_{0}^{\infty} u_{w} d y
$$

Substituting Eqs. (8.4), (8.5), and (8.12) in the limit of small $c_{f}$, leaves

$$
Q_{\text {wall }}=\pi D h_{w} U_{o} \sqrt{d_{o}} \psi_{1,0} \beta_{w} \sqrt{z+z_{o}}
$$

where

$$
\psi_{1,0} \equiv \int_{0}^{\infty} f_{w}\left(\eta_{w}\right) d \eta_{w}=1.098
$$

from Eq. (8.7). The total volumetric flow rate including all of the entrained fluid by the jet is

$$
Q_{\text {total }}=Q_{\text {central }}+Q_{\text {wall }}
$$

These expressions inform the fraction of the vessel mixed, the time for mixing to be achieved throughout the vessel, and the number of pulses required to mix the complete vessel, each under a variety of conditions and circumstances pertinent to the vessel in question. 
Table 9.1 summarizes projected volumes of mixing in the proposed $16 \mathrm{ft}$ vessel at the moment the full jet reaches the fluid surface. These projections are based on Eq. 9.5 or 9.6 with parameters $\eta_{c}^{99} \beta_{c}$ or $R_{j e t}$ from prior sections of this report based on historical data from which the radial spread of the jet was estimated. Projection from historical data is necessitated because the radial distribution of axial velocities was not measured in testing in the eight foot vessel. The calculated volume includes only the volume of the jet at the moment surface flow is achieved. The table shows that the portion of the vessel directly mixed by the jet at the moment surface flow is achieved ranges from $<0.5 \%$ to nearly $75 \%$ of the vessel volume depending on the flow conditions and slurry characteristics. Blending in the vessel head alone constitutes $15.2 \mathrm{~m}^{3}$ or $18.02 \%$ of the vessel blending.

Specifically, Table 9.1 considers the glass beads for which the axial velocity profiles were measured as a function of the radius. As the solids loading increases at $10 \mu \mathrm{m}$, the radial spread of velocities narrows so that the fraction of the vessel mixed decreases. The extent to which larger solids would further decrease the fraction of the vessel blended remains unknown. Additional flow after the initial cone of the jet is established is given in the final column of Table 9.1. This flow can be very significant, adding cubic meters of flow per second. However, this flow too diminishes in the presence of significant solids loadings. The lowest prediction is for KB slurry in the laminar flow regime. Here the flow is channeled and does not spread like a free jet such that even though this flow reached the surface it did not significantly mix the remainder of the vessel. This laminar flow model was shown in Section 6.3 to provide a good fit to velocity data versus elevation (see Figure 6.6) from testing in the $8 \mathrm{ft}$ vessel even though the flow was turbulent at the base of the upwell, but Eq. 6.21 suggests that the viscous stresses will be more important in the $16 \mathrm{ft}$ vessel (the ratio of viscous to yield stresses is 2.2 for $\mu_{o}=0.030 \mathrm{Pas}, U_{o}=12 \mathrm{~m} / \mathrm{s}, \beta_{c}=0.0551, \tau_{o}=30 \mathrm{~Pa}$, and $d_{o}=4$ inches in Eq. (6.21)), which may increase the radial spread of the jet relative to the $8 \mathrm{ft}$ vessel.

For the wall jet in the $8 \mathrm{ft}$ vessel test data some turbulent flows reached the probe 34 inches above the seam (see Section 8). Were the wall jet to achieve the same relative height in the $16 \mathrm{ft}$ vessel, this jet would mix an additional $6.2-28.7 \%$ (14\% average) of the total vessel volume. However, even in the $8 \mathrm{ft}$ vessel many of the PJM pulses did not establish a measurable jet to this level and the wall jet in the KB slurry was typically negligible above the seam, so the extent to which the upward wall jet may contribute to vessel mixing remains uncertain but likely smaller (the downward flow along the wall not analyzed herein may be a larger contributor to vessel mixing than the upward jet). 
Table 9.1. Projected Volumes of Mixing at Surface Flow Achieved by the Central Upwell Alone per Pulse in a $16 \mathrm{ft} \mathrm{Vessel}^{(\mathrm{a})}$

\begin{tabular}{|c|c|c|c|c|}
\hline Basis & Condition $^{(b)}$ & $\begin{array}{c}V_{\text {central }} \\
\left(\mathrm{m}^{3}\right)\end{array}$ & $\begin{array}{c}V_{\text {central }} / V_{\text {total }} \\
(\%)\end{array}$ & $\begin{array}{l}Q_{\text {central }} \\
\left(\mathrm{m}^{3} / \mathrm{s}\right)\end{array}$ \\
\hline Water (Bontha et al. 2003a) & Turbulent Dispersive $^{(\mathrm{c})}$ & 16.16 & 19.4 & 4.69 \\
\hline Water (Bontha et al. 2003b) & Turbulent Dispersive $^{(\mathrm{c})}$ & 13.20 & 15.6 & 2.12 \\
\hline $10 \mu \mathrm{m}$ glass beads $5 \mathrm{wt} \%$ & Turbulent Dispersive $^{(\mathrm{c})}$ & 21.34 & 25.6 & 3.60 \\
\hline $10 \mu \mathrm{m}$ glass beads $20 \mathrm{wt} \%$ & Turbulent Dispersive $^{(\mathrm{c})}$ & 15.22 & 18.2 & 2.44 \\
\hline $35 \mu \mathrm{m}$ glass beads $5 \mathrm{wt} \%$ & Turbulent Dispersive $^{(\mathrm{c})}$ & 7.86 & 9.41 & 1.10 \\
\hline $35 \mu \mathrm{m}$ glass beads $20 \mathrm{wt} \%$ & Turbulent Dispersive $^{(\mathrm{c})}$ & 9.44 & 11.3 & 0.956 \\
\hline KB slurry & Turbulent Dispersive $^{(\mathrm{c})}$ & 4.80 & 5.75 & 0.518 \\
\hline Laponite (1.44 ft vessel) & Turbulent Dispersive $^{(\mathrm{d})}$ & 32.0 & 38.4 & - \\
\hline Laponite (2.82 ft vessel) & Turbulent Dispersive $^{(\mathrm{d})}$ & 25.0 & 29.9 & - \\
\hline Laponite (12.75 ft vessel) & Turbulent Dispersive $^{(\mathrm{d})}$ & 26.5 & 31.7 & - \\
\hline Laponite (average for all vessels) & Turbulent Dispersive $^{(\mathrm{d})}$ & 36.8 & 44.0 & - \\
\hline Laponite (1.44 ft vessel) & Turbulent Dispersive With Vortex ${ }^{(\mathrm{e})}$ & 7.27 & 8.71 & - \\
\hline Laponite (2.82 ft vessel) & Turbulent Dispersive With Vortex ${ }^{(e)}$ & 10.6 & 12.7 & - \\
\hline Laponite (average for all vessels) & Turbulent Dispersive With Vortex ${ }^{(\mathrm{e})}$ & 12.6 & 15.1 & - \\
\hline Laponite (1.44ft vessel) & Constant Radius $^{(\mathrm{f})}$ & 23.7 & 28.3 & - \\
\hline Laponite (2.82 ft vessel) & 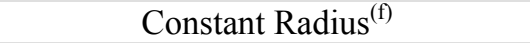 & 12.2 & 14.6 & - \\
\hline Laponite (12.75 ft vessel) & Constant Radius ${ }^{(f)}$ & 7.15 & 8.56 & - \\
\hline Laponite (average for all vessels) & Constant Radius $^{(\mathrm{f})}$ & 13.4 & 16.0 & - \\
\hline KB slurry (1.44 ft vessel) & Constant Radius ${ }^{(\mathrm{g})}$ & 62.4 & 74.8 & - \\
\hline KB slurry (2.82 ft vessel) & Constant Radius ${ }^{(\mathrm{g})}$ & 10.5 & 12.5 & - \\
\hline KB slurry (2.82 ft vessel) & Constant Radius ${ }^{(\mathrm{g})}$ & 5.32 & 6.37 & - \\
\hline KB slurry (12.72 ft) & Constant Radius ${ }^{(\mathrm{g})}$ & 5.19 & 6.21 & - \\
\hline KB slurry (12.72 ft) & Constant Radius $^{(\mathrm{g})}$ & 13.0 & 15.5 & - \\
\hline KB slurry (12.72 ft) & Constant Radius ${ }^{(\mathrm{g})}$ & 12.8 & 15.3 & - \\
\hline KB slurry (run sheet 3 ) & Laminar $^{(\mathrm{h})}$ & 11.2 & 13.4 & - \\
\hline KB slurry (run sheet 3 ) & Laminar $^{(\mathrm{h})}$ & 15.7 & 18.8 & - \\
\hline KB slurry (12.75 ft vessel) & Laminar $^{(\mathrm{h})}$ & 0.37 & 0.44 & - \\
\hline KB slurry (12.75 ft vessel) & Laminar $^{(\mathrm{h})}$ & 0.70 & 0.84 & - \\
\hline
\end{tabular}

(a) Approximate vessel dimensions, volume, and nozzle diameter from Fink and Peterson (2015). Does not account for suction. Based on elevations between seam/knuckle/tangent and nominal fill height.

(b) Sourced from velocity profiles for jets that both did and did not upwell to the surface or achieve breakthrough unless specifically noted.

(c) $\beta_{c}$ and $z_{o} / d_{o}$ from Table $2.1, \eta_{c}^{99}$ from Table 3.1. Assumes a nozzle velocity of $12 \mathrm{~m} / \mathrm{s}$.

(d) $\eta_{c}{ }^{99} \beta_{c}$ and $z_{o} / d_{o}$ from Table 6.1, Eq. 6.7. The value of $\beta_{c}$ is not known independently so $Q_{\text {central }}$ cannot be estimated directly.

(e) $\eta_{c}^{99} \beta_{c}$ and $z_{o} / d_{o}$ from Table 6.1, Eq. 6.15. The value of $\beta_{c}$ is not known independently so $Q_{\text {central }}$ cannot be estimated directly. Additional mixing volume may arise from the leading vortex not included here.

(f) $R_{j e t} / d_{o}$ from Table 6.1, Eq. 6.27. Assumes a simple right cylinder of radius $R_{j e t}$ from seam to fluid surface.

(g) $R_{j e t} / d_{o}$ from Table 6.2. Assumes a simple right cylinder of radius $R_{j e t}$ from seam to fluid surface. Sourced only from flows that achieved breakthrough.

(h) $R_{j e t} / d_{o}$ from Table 6.3. Assumes a simple right cylinder of radius $R_{j e t}$ from seam to fluid surface. The top in each pair achieved breakthrough, whereas the bottom of each pair did not.

Table 9.1 also shows that the fraction of the vessel mixed varies considerably depending on the flow regime assumed. Unsurprisingly, the laponite and KB slurries evaluated in the $1.44 \mathrm{ft}$ vessel usually had a larger volume of mixing than the other vessels, because the ratio of the yield Reynolds number to the jet Reynolds number was larger in testing of that vessel than in the other vessels (see Section 6). This ratio of Reynolds numbers is characteristic of the ratio of the viscous to yield stresses so that when this ratio is large, the jets experience less confinement than when this ratio is small (governed by the yield stresses). 
However, where this ratio is larger, the jet Reynolds number introduces a scale dependence. Indeed, this may present an interesting case where marginally losing certainty in scaling between vessels (until the right equations have been formulated and solved) may be compensated by a substantially larger volume of mixing. Yet, how these two effects balance to create an optimal mixing environment in the $16 \mathrm{ft}$ vessels remains unexplored.

Notably, the ratio of viscous stresses to yield stresses for the 8 and $16 \mathrm{ft}$ vessels is larger than that in much of the historical $12.75 \mathrm{ft}$ vessel testing. This suggests that the volumes of mixing projected from the $1.44 \mathrm{ft}$ vessel may be a better projection of mixing than the larger vessel testing. Additional analysis is necessary to quantify these effects. However, Section 6.3 showed that a laminar flow model appeared to fit the data from the $8 \mathrm{ft}$ testing well, leaving significant uncertainty in the anticipated volume of mixing due to a gap in the available models, which can in large measure be filled by coupling momentum and energy conservation together and benchmarking against relevant test data.

The estimates in this section depend on four parameters characterizing the radial and lateral distributions of the jets: $\eta_{c}{ }^{99}, \eta_{w}{ }^{99}, \beta_{c}$, and $\beta_{w}$. These parameters are seldom a focus of testing. Measurements in the $16 \mathrm{ft}$ vessel represent an opportunity to ascertain their values more precisely, particularly in the presence of significant solids loadings. Moreover, the wide range of estimates for the volume of mixing from the same tests in Table 9.1 highlights the difficulty in extrapolating vessel mixing performance from limiting conditions using limited data sources. Properly coupling momentum and energy balances together with the appropriate turbulence models has the potential to substantially simplify and consolidate these results. By so doing, straightforward and user friendly expressions may be obtained and increase confidence in the design and performance of PJM mixed vessels.

\subsection{Why the Jet Cone Alone May Underestimate the Mixing Volume}

The jet cone approach remains the most reliable analytical path to estimate the volume of the vessel mixed (until the momentum and energy equations are coupled together to give a more accurate representation of the sheared volume). However, other factors may play a role that could enlarge the volume estimate. First, analytical derivations of the velocity profile in the radial direction for both circular and wall jets find that the radial velocity far from the jet center does not decay to zero. For example, Table 2-1 of Rajaratnam (1976) shows that the radial velocity $v$ asymptotes to approximately $-1 / 3$ when normalized on the product $u_{m, c}$ and a constant that evaluates to 0.066 ; see discussion above Eq. 2-83, Rajaratnam (1976). Combined, this implies that this radial inward (from the minus sign) velocity remains finite. This entrainment velocity may or may not represent an asymptotic approximation, but to the extent physical, this non-zero radial velocity will marginally enhance lateral blending even if only by a few percent of the centerline axial velocity. Velocity probes were not positioned far enough from the vessel centerline to quantify this velocity either historically or in recent testing in the $8 \mathrm{ft}$ vessel, likely because it was assumed to be small. It may not be large, but suggests a conservatism in the calculations above.

In the presence of solids loadings sufficient to induce negative buoyancy, the presence of a fluid dynamic cloud height forms a cap that restricts or attenuates the vertical extent of jet flow. The volume of mixing may then be estimated from the jet by replacing $z_{u}$ in the expressions above with $z_{\text {cloud }}$. Lower cloud heights would lead to smaller truncated jet cones and presumably decrease the volume of mixing. However, lateral mixing may be enhanced substantially in these flows below the cloud height. Consider, 
for instance, the flow entering the central upwell. If this flow cannot progress vertically, conservation of mass demands significant and substantial fluid flow laterally. One may not be surprised then to find a dramatic increase in shearing below the cloud height at elevations above the seam, were they measured. Similarly, stress coupling between the fluid below the cloud height and the fluid above the cloud height may induce velocities there, including the waves identified by Kuhn et al. (2013) and the rather small velocities along the centerline measured in the $8 \mathrm{ft}$ testing with Newtonian simulants. It is also possible that downward flow peripheral to the jet, well recognized in fountain flows (even if they scale differently), may enhance mixing, but downward flows in the presence of a cloud height peripheral to the primary flow do not appear to have been measured quantitatively to date.

In the presence of a significant yield stress, experimental data from the $8 \mathrm{ft}$ vessel suggests that the steady-state jet cone may not completely define the volume of mixing. Figure 9.1 shows the vertical velocities measured by a probe 1.1 inches off center (Valeport 0580 ) and at 18.9 inches from the centerline (Valeport 0576) are both 34 inches above the seam at nozzle velocities of 12 and $7.9 \mathrm{~m} / \mathrm{s}$ (Bontha et al. 2015). In Figure 9.1a at the higher velocity, both probes show an initial velocity commensurate with bulk motion. Then, at approximately $4 \mathrm{~s}$, both probes register a sharp velocity rise as the initial vortex passes with the Valeport probe at the centerline indicating a velocity rise approximately $0.11 \mathrm{~s}$ earlier at the inflection point. At the same moment, a spike in the horizontal velocity (oriented azimuthally) as measured 18.9 inches from the centerline also indicates the passage of the initial vortex.

After the initial pulse passes, the velocity along the centerline remains strong, but the velocity 18.9 inches from the centerline all but decays. In fact, the figure shows that there is a small but non-zero velocity in the opposite direction, suggesting downward flow 18.9 inches from the centerline. Therefore, the primary jet flow does not extend out that far, even though the initial vertex does. The downward flow and the influence of turbulence suggest that the initial vertex mobilized this region of flow outside the main jet flow and that it remains mobilized due to passing transient vortices.

At the end of the pulse, the horizontal velocity again spikes at 18.9 inches from the centerline, suggestive of the channel closing. It is not surprising that the initial vortex would be larger than the main jet flow, as Johari et al. (1997) found that the starting entrainment of jets in Newtonian fluids was approximately $30 \%$ larger than in the main jet flow. Kuhn et al. (2013) estimated the radius of leading vortex to be approximately twice the radius of the jet below. In contrast, at the lower velocity of $7.9 \mathrm{~m} / \mathrm{s}$, the initial ring vortex does not pass, the downward flow after the jet traverses the centerline at that elevation is absent peripherally to the jet, and the channel does not experience a significant radial flow at the end of the pulse. Combined, this suggests that the jet does not extend out to 18.9 inches at this lower velocity.

In the KB slurries in WTP vessels or more generally in the full range of non-Newtonian fluids proposed for processing by the WTP, the full radial spread of the leading vortex remains unknown, but depends on nozzle velocity and may play a significant role in vessel mixing beyond that anticipated from the main jet flow alone. 

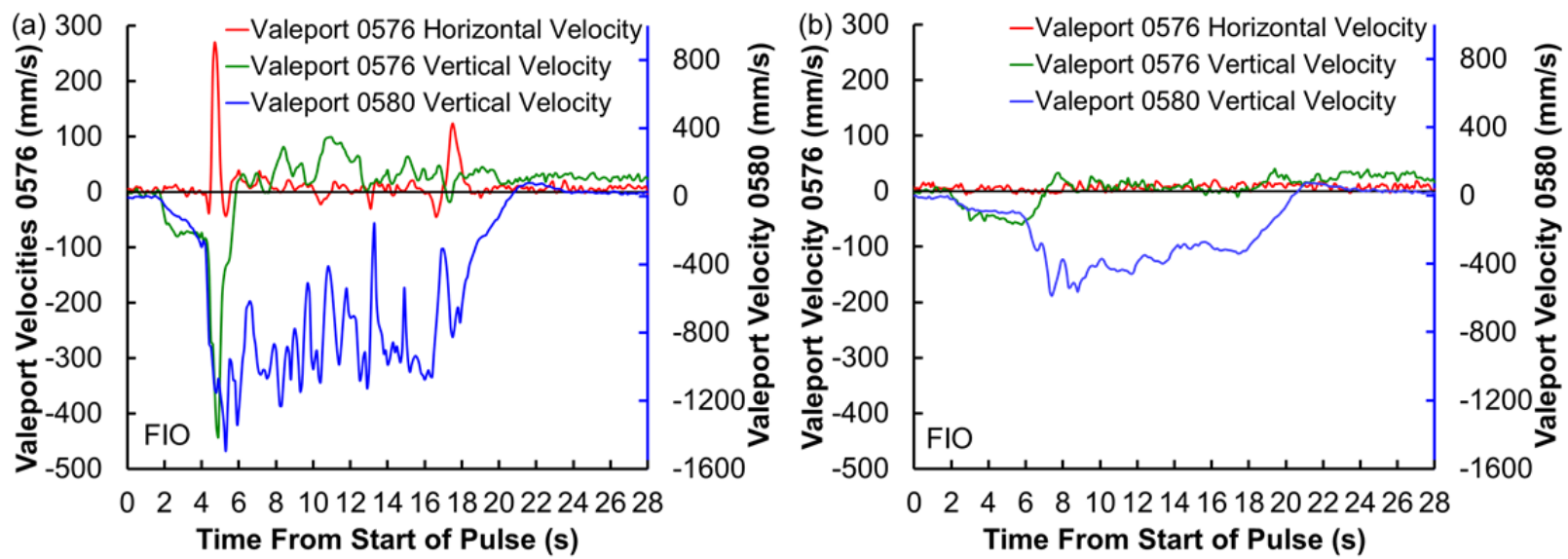

Figure 9.1. Fluid Velocities in the $8 \mathrm{ft}$ Vessel in Tests (a) 3-8.5.1 (time stamp 140349) with $U_{o}=12 \mathrm{~m} / \mathrm{s}$ and (b) 3-8.1.44 (time stamp 032930) with $U_{o}=7.9 \mathrm{~m} / \mathrm{s}$ with Nozzle Velocities Both Measured 34 inches Above the Seam and 18.9 inches and 1.1 inches From the Vessel Center with Valeport Probes 0576 and 0580, respectively. A 2.25 s delay in processing the Valeport probe information may affect the timing of these curves relative to the pulse start (Bontha et al. 2015).

\subsection{Summary}

This section estimates volumes of mixing based primarily on the volume and flow rate of the central upwell. Because the axial and radial lengths of the central upwell may differ dramatically, observation of surface flow does not necessarily ensure significant mixing peripheral to the jet, particularly through non-Newtonian simulants. This analysis remains incomplete because the radial distribution of axial velocities has only rarely been measured, the size of the leading vortex has not been evaluated, and subsequent radial flows along the surface of the fluid and down the vessel walls have not been modeled. 


\subsection{Key Findings}

This section lists the 15 key findings of this report, followed by an analysis that identifies gaps or opportunities.

\subsection{Key Findings}

1. The central upwell behaves as a free jet in the absence of body and surface forces as supported by consideration of suitable test data.

2. When the axial velocity of this free jet is scaled on the jet nozzle velocity and the elevation on the nozzle diameter, the data collapse onto curves that depend only on the jet coefficient and the location of a virtual origin with respect to the vessel bottom. These parameters have been extracted from historical and $8 \mathrm{ft}$ testing with multiple simulants at multiple scales.

3. The radial distribution of velocities is essential to quantify flow within mixing vessels. The fractional width of the jet was approximately $10 \%$ to $20 \%$ of the vessel width in the $12.75 \mathrm{ft}$ vessel. Available data leave uncertainty in the radial spread of the jet of approximately a factor of three.

4. The Poirier-Etchells criterion presents a cutoff velocity for jet turbulence that is approximately predictive of surface flow when coupled with the free jet equation.

5. Conservation of momentum may be used to predict jet coefficients for free jets. A geometric approximation may be used to estimate how the central upwell depends on the number of PJMs within a mixing vessel. Shear forces along the vessel bottom only weakly influence the upwell flows. Predicted jet coefficients for a free jet typically remain within a factor of three of measured jet coefficients for Newtonian simulants.

6. A general framework that combines momentum and species conservation establishes relationships among jet momentum, buoyancy, and yield stresses. The framework is sufficiently general to permit simultaneous evaluation of buoyancy and yield stresses (e.g., for simulants with low yield stresses and density stratification).

7. Jet flow governs the central upwell with and without solids until the negative buoyancy force becomes influential. The Froude number based on nozzle diameter without density contributions collapses the data from $8 \mathrm{ft}$ vessel tests regardless of pulse volume fraction, PJM firing sequence, or Newtonian simulant with separate critical Froude numbers for each nozzle diameter. A Froude number based on tank diameter would not lead to a single critical Froude number. The Froude number alone in its various forms, therefore, cannot reliably predict scale-up of flows below the upper surface because the critical Froude number varies with at least nozzle diameter.

8. A new cloud height model was developed from first principles and validated against the $8 \mathrm{ft}$ vessel tests with the two-part simulant. This model requires four parameters: the Froude number, the jet coefficient, the ratio of the density at the base of the upwell to the density far from the jet centerline, and the ratio of the depth of the virtual origin below the vessel bottom to the nozzle diameter. The first is clearly scale dependent, and the second is scale independent. How the latter two scale between vessel sizes remains unclear. When these are known, this cloud height model predicted surface flow.

9. Conservation of energy alone suggests that in a yield stress material, the jet initially expands and then contracts. Without solving the coupled momentum and energy conservation equations together, three 
limiting cases are considered. First, an expanding turbulent jet predicts the critical elevation at which the velocity ceases due to insufficient jet momentum. This model, validated against "cavern" height data in laponite, collapses the data from multiple scales onto a single curve. Second, the turbulent confined jet of constant radius shows that the jet radius increases as the yield Reynolds number (defined on the Bingham yield stress, not the shear strength) increases until it is large enough that viscous forces become influential. Analysis shows that the upwell flow loses turbulence and becomes laminar. Third, when upwell flow is laminar (supported by measured radial velocity profiles), the square of the ratio of the centerline velocity to the nozzle velocity decays linearly with the elevation scaled on nozzle diameter. The third case represents velocity data in the $8 \mathrm{ft}$ vessel well at $8 \mathrm{~m} / \mathrm{s}$. Further work is needed to consider fully coupled momentum and energy to add fidelity to these relationships.

10. Flows within mixing vessels holding yield stress materials may be independent of geometric scaling at constant nozzle velocity when the yield Reynolds number is sufficiently small so long as the virtual origin does not shift between scales. When the ratio of the yield Reynolds number to jet Reynolds number, which represents the ratio of viscous stresses to yield stresses, is order one, the jet Reynolds number becomes influential. For the $16 \mathrm{ft}$ vessel, this ratio is order one. The jet Reynolds number depends on geometric scale, so that scaling between vessels may depend on geometric scale. See Wells et al. (2015) for further analysis.

11. Velocities and diameters of the upwell flows in the presence of yield stress materials are presented, laying important groundwork for evaluating the surface and downwell flows that govern mixing/blending outside the upwell flow (i.e., the remainder of mode three mixing). The radial velocity distribution determines the local effective viscosity, which in turn governs hydrogen release.

12. The rate at which the upwelling jet propagates or penetrates toward the fluid surface (termed jet celerity) determines whether the jet achieves surface flow or not.

13. Simply integrating the centerline jet velocity underestimates the time a free jet takes to rise to the fluid surface. The time-dependent conservation of momentum equation was solved to show that the radial distribution of velocities serves as a key correction factor. Including the radial distributions improves prediction of surface flow. The time for the jet to transit from the vessel seam, tangent, or knuckle to the fluid surface comprises a strong majority of jet transit time.

14. Conservation of momentum shows jet flow along the walls to be independent of geometric scaling (the vessel diameter is divided by the nozzle diameter), but the velocity decays with the inverse of the square root of the elevation instead of with the inverse of the elevation directly. Even though more of the PJM momentum flows toward the wall, the momentum spreads out over the perimeter and is countered by a down flow, so that the jet flow along the wall is typically both weaker and slower than the central upwell. Therefore, this report focuses on the central upwell.

15. Jet physics permits estimation of the volume mixed (i.e., sheared) by the central upwell during a single pulse at the moment surface flow is achieved (lateral flow not included). Mixing caused by only the central upwell as it achieves surface flow is estimated to vary from $<0.5 \%$ to $\sim 75 \%$ depending on the radial distribution of velocities (Table 9.1). Additional flow may proceed after surface flow has been achieved until the end of the PJM pulse, in other parts of the vessel (below seam, $18 \%$; wall upwell, typically $<15 \%$ ), and due to the leading vortex (a measurement opportunity in the $16 \mathrm{ft}$ vessel with radially distributed velocity probes). 


\subsection{Gap Analysis}

This analysis identifies the following gaps or opportunities.

1. Radial distributions of axial velocities need to be measured in vessels other than the $12.75 \mathrm{ft}$ test vessel for both Newtonian and non-Newtonian simulants. This is the most pervasive need. These feed directly into estimates of the jet coefficients, jet dimensions, and estimates of the volume sheared by a single pulse, which in turn affect cloud height, "cavern" height, and jet celerity models that predict surface flow. How the radial distributions vary with elevation remains unclear, but is essential to predicting surface flow and lateral shearing. Radial distributions that vary with axial position may be predicted and compared to revised models that couple energy and momentum conservation, because they are not independent in the presence of buoyancy forces and yield stresses, unlike the free jet.

2. To use the cloud height model to predict scalability, models are needed for the slurry densities at the base of the upwell, the slurry density far from the center of the upwell, and the depth of the virtual origin, preferably from first principles benchmarked against experiments. When available the combined model will permit prediction of conditions that satisfy mixing requirements.

3. There is a need for a continuous, unified model for flow in the presence of yield stresses that accounts for the influence of radial distributions that surely change with elevation and the turbulent-to-laminar transition. Coupling energy and momentum equations will likely result in simpler quantitative equations, replacing the intricate derivations herein.

4. Jet celerity has been shown to quantify surface flow for the $8 \mathrm{ft}$ vessel tests and a predictive equation is available for the free jet. Modifications for buoyancy and yield stresses are needed to make this celerity analysis a predictive tool.

5. The viscosity or shear stress-shear rate responses of simulants and actual waste have not been well characterized at low shear rates. Characterizing non-Newtonian materials under these conditions is important because it determines a boundary condition that governs whether surface flow is achieved in these materials.

6. The new cloud height model has been benchmarked against one simulant in one vessel size. Additional benchmarking in other vessels with other simulants will improve confidence in using this model. The jet density distribution should also be benchmarked against experimental data.

7. Additional flows that have not been considered include lateral flows across the vessel surface or "cloud surface," downwell flows along the vessel wall, and "fan" jets that arise between PJMs.

8. The analysis herein forms the basis of analytical models of mode three flow and hydrogen release. There is an opportunity to address these important aspects of mixing based on this analysis. 



\subsection{References}

Abramovich GN. 1963. The Theory of Turbulent Jets. The M.I.T. Press, Cambridge Massachusetts. Agelin-Chaab M and MF Tachie. 2011. "Characteristics and Structure of Turbulent 3D Offset Jets." International Journal of Heat and Fluid Flow 32:608-620.

Bajpai S and MS Tirumkudulu. 2008. "An experimental study of impulsively started turbulent axisymmetric jets.” The European Physical Journal B 61:293-297.

Bamberger JA, PA Meyer, JR Bontha, CW Enderlin, DA Wilson, AP Poloski, JA Fort, ST Yokuda, HD Smith, F Nigl, M Friedrich, DE Kurath, GL Smith, JM Bates, and MA Gerber. 2005. Technical Basis for Testing Scaled Pulse Jet Mixing Systems for Non-Newtonian Slurries. PNWD-3551, WTP-RPT-113, Rev. 0, Battelle-Pacific Northwest Division, Richland, Washington.

Barlow RS and CQ Morrison. 1990. "Two-phase velocity measurements in dense particle-laden jets." Experiments in Fluids 9:93-104.

Bejan A, S Ziaeil, and S Lorente. 2014. "Evolution: Why all plumes and jets evolve to round cross sections." Scientific Report 4:4730:1-5.

Beltaos S. 1975. "Oblique Impingement of Circular Turbulent Jets.” Journal of Hydraulic Research $14: 17-36$.

Beltaos S and N Rajaratnam. 1977. "Impingement of Axisymmetric Developing Jets." Journal of Hydraulic Research 15:311-326.

Bird RB, WE Stewart, and EN Lightfoot. 2002. Transport Phenomena, Second Edition. John Wiley \& Sons, New York.

Birkhoff G and EH Zarantonello. 1957. Jets, wakes, and cavities. Academic Press, New York.

Bontha JR, TE Michener, DS Trent, JM Bates, and MD Johnson. 2003a. Development and Assessment of the TEMPEST CFD Model of the Pulsed Jet Mixing Systems. PNWD-3261, WTP-RPT-061, Rev. 0, Battelle-Pacific Northwest Division, Richland, Washington.

Bontha JR, JM Bates, CW Enderlin, and MG Dodson. 2003b. Large Tank Experimental Data for Validation of the FLUENT CFD Model of Pulsed Jet Mixers. PNWD-3303, WTP-RPT-081, Rev. 0, Battelle-Pacific Northwest Division, Richland, Washington.

Bouchet G, E Climent, and A Maurel. 2002. "Instability of a confined jet impinging on a water/air free surface." Europhysics Letters 59:827-833.

Camenen B. 2007. "Simple and General Formula for the Settling Velocity of Particles." Journal of Hydraulic Engineering - ASCE 133(2):229-233. 
Cai J, DZ Zhu, and N Rajaratnam. 2012. “Observations on sand jets in viscoplastic fluids." Theoretical and Applied Mechanics Letters 2:05200:1-6.

Chatzimina M, C Xenophontos, GC Georgiou, I Argyropaidas, E Mitsoulis. 2007. "Cessation of annular Poiseuille flows of Bingham plastics.” Journal of Non-Newtonian Fluid Mechanics 142:135-142.

Chun J, AP Poloski, and EK Hansen. 2010. "Stabilization and control of rheological properties of $\mathrm{Fe}_{2} \mathrm{O}_{3} / \mathrm{Al}(\mathrm{OH})_{3}$-rich colloidal slurries under high ionic strength and $\mathrm{pH}$." Journal of Colloid and Interface Science 348:280-288.

Crapper PF and WD Baines. 1976. "Non Boussinesq Forced Plumes." Atmospheric Environment $11: 415-420$.

Dawson DA and O Trauss. 1966. "Mass transfer in a turbulent radial wall jet." The Canadian Journal of Chemical Engineering 44:121-129.

Deen WM. 2012. Analysis of Transport Phenomena. Second Edition. Oxford University Press, New York.

Enderlin CW, MG Dodson, F Nigl, J Bontha, and JM Bates. 2003. Results of Small-Scale Particle Cloud Tests and Non-Newtonian Fluid Cavern Tests. PNWD-3360, WTP-RPT-078, Battelle-Pacific Northwest Division, Richland, Washington.

Fink SD and K Peterson. 2015. Hold Points 1 and 2 Release for the Standard High Solids Vessel Design Test Vessel. 24590-WTP-ES-ENG-15-013, Rev. 0, Bechtel National, Inc., Richland, Washington.

Gauglitz PA, BE Wells, JA Fort, and PA Meyer. 2009. An Approach to Understanding Cohesive Slurry Settling, Mobilization, and Hydrogen Gas Retention in Pulsed Jet Mixed Vessels. PNNL-17707, WTP-RPT-177, Rev. 0, Pacific Northwest National Laboratory, Richland, Washington.

Gauglitz PA, BE Wells, JA Bamberger, JA Fort, J Chun, and JJ Jenks. 2010. The Role of Cohesive Particle Interactions on Solids Uniformity and Mobilization During Jet Mixing: Testing Recommendations. PNNL-19245, Pacific Northwest National Laboratory, Richland, Washington.

Gordon P. 2003. Principles of Phase Diagrams in Material Systems. Reprint of original 1968 edition by McGraw-Hill, Ceramic Book and Literature Service, Ohio.

Herting DL. 2012. Simulant Qualification for Computational Fluid Dynamics Testing. 24590-WTP-RPT-RT-12-002, Rev. 0, Bechtel National, Inc., Richland, Washington.

Jenny P and B Muller. 1998. "Rankine-Hugoniot-Riemann Solver Considering Source Terms and Multidimensional Effects." Journal of Computational Physics 145:575-610.

Kuhn WL, DR Rector, SD Rassat, CW Enderlin, MJ Minette, JA Bamberger, GB Josephson, BE Wells, and EJ Berglin. 2013. Scaling Theory for Pulsed Jet Mixed Vessels, Sparging, and Cyclic Feed Transport Systems for Slurries. PNNL-22816, Pacific Northwest National Laboratory, Richland, Washington. 
Kurath DE, PA Meyer, JR Bontha, AP Poloski, JA Fort, WH Combs, WC Buchmiller, ID Welch, and MD Bleich. 2007. Assessment of Pulse Tube Mixing for Vessels Containing Non-Newtonian Slurries. PNWD-3827, WTP-RPT-155, Rev. 0, Battelle-Pacific Northwest Division, Richland, Washington.

Lamb H. 1945. Hydrodynamics. Reprint of original 1932 publication by Cambridge University Press, of the work first published in 1879, Dover Publications, New York.

Landa P, V Ushakov, and J Kurths. 2005. "Linear Instability of a Slowly Divergent Planar Jet." In Progress in Turbulence, eds. J Peinke, A Kittel, S Barth, and M Oberlack. Springer, New York, pp. 27-30.

Larson RG. 1999. The Structure and Rheology of Complex Fluids. Oxford University Press, New York.

Lin XB. 2000. “Generalized Rankine-Hugoniot Condition and Shock Solutions for Quasilinear Hyperbolic Systems." Journal of Differential Equations 168:321-354.

Man W, D Aleksandar, FH Stillinger, MT Sullivan, WB Russel, D Heeger, S Inati, S Torquato, and PM Chaikin. 2005. "Experiments on Random Packings of Ellipsoids." Physical Review Letters 94:198001:1-4.

Masuyama T, K Sato, T Kawashima, and K Noda. 1980. "Pressure Loss of Bingham Plastic Fluid Flow in Pipes.” Journal of the Mining and Metallurgical Institute of Japan 96:385-389.

Meacham JE, SJ Harrington, JS Rodriguez, VC Nguyen, JG Reynolds, BE Wells, GF Piepel, SK Cooley, CW Enderlin, DR Rector, J Chun, A Heredia-Langner, and RF Gimpel. 2012. One System Evaluation of Waste Transferred to the Waste Treatment Plant. RPP-RPT-51652, Washington River Protection Solutions LLC, Richland, Washington.

Melville WK and KNC Bray. 1979a. "A Model of the Two-Phase Turbulent Jet." International Journal of Heat and Mass Transfer 22:647-656.

Melville WK and KNC Bray. 1979b. "The Two-Phase Turbulent Jet." International Journal of Heat and Mass Transfer 22:279-287.

Meyer PA, DE Kurath, JA Bamberger, AW Etchells and SM Barnes. 2006. "Scaling Laws for Reduced-Scale Tests of Pulse Jet Mixing Systems in Non-Newtonian Slurries: Mixing Cavern Behavior." 2006 Waste Management Symposium, Tucson, Arizona, WM Symposia, Inc. Available online at http://www.wmsym.org/abstracts/2006/index.html.

Meyer PA and AW Etchells. 2007. "Mixing with Intermittent Jets with Application in Handling Radioactive Waste Sludges.” Chemical Engineering Research and Design 85(5):691-696.

Meyer PA, JA Bamberger, CW Enderlin, JA Fort, BE Wells, SK Sundaram, PA Scott, MJ Minette, GL Smith, CA Burns, MS Greenwood, GP Morgan, EBK Baer, SF Snyder, M White, GF Piepel, BG Amidan, and A Heredia-Langner. 2012. Pulse Jet Mixing Tests With Noncohesive Solids. PNNL-18098, WTP-RPT-182, Rev. 1, Pacific Northwest National Laboratory, Richland, Washington. 
Mitsoulis E. 2007. "Flows of Viscoplastic Materials: Models and Computations." Rheology Reviews 2007:135-178.

Mostafa AA and HC Mongia. 1988. "On the interaction of particles and turbulent fluid flow." International Journal of Heat and Mass Transfer 31:2063-2075.

Phares DJ, GT Smedley, and RC Flagan. 2000. "The wall shear stress produced by the normal impingement of a jet on a flat surface." Journal of Fluid Mechanics 418:351-375.

Plawsky JL. 2001. Transport Phenomena Fundamentals. Marcel Dekker, New York.

Poloski AP, PR Bredt, JW Chenault, RG Swoboda. 2003. Rheological and Physical Properties of AZ-101 HLW Pretreated Sludge and Melter Feed. PNWD-3366, WTP-RPT-096, Rev. 0, Battelle-Pacific Northwest Division, Richland, Washington.

Poloski AP, PA Meyer, LK Jagoda, and PR Hrma. 2004. Non-Newtonian Slurry Simulant Development and Selection for Pulse Jet Mixer Testing. PNWD-3495, WTP-RPT-111, Rev. 0, Battelle-Pacific Northwest Division, Richland, Washington.

Poreh M, YG Tsuei, and JE Cermak. 1967. "Investigation of a Turbulent Radial Wall Jet." Journal of Applied Mechanics June 1967:457-463.

Powell MP, Y Onishi, and R Shekkariz. 1997. Research on Jet Mixing of Settled Sludges in Nuclear Waste Tanks at Hanford and Other DOE Sites: A Historical Perspective. PNNL-11686, Pacific Northwest National Laboratory, Richland, Washington.

Rajaratnam N. 1976. Turbulent Jets. Elsevier, Amsterdam.

Rao VK. 1980. "A Note on Mass Transfer in Turbulent Wall Jets." International Journal of Heat and Mass Transfer 23:1690-1693.

Russel WB, DA Saville, and WR Schowalter. 1989. Colloidal Dispersions. Cambridge University Press, New York.

Sangras R and GM Faeth. 1999. Buoyant Turbulent Jets and Plumes: III. Round Turbulent Nonbuoyant Starting Jets and Puffs and Buoyant Starting Plumes and Thermals. Annual Report for Grant No. 0NANB8D0081, Report Number GDL/GMF-99-03, National Institute of Standards and Technology, Washington, D.C.

Shekarriz A, G Douillard, T Weir, and CD Richards. 1995a. "Rheology and Reynolds Number Effects on the Velocity Field of a Pseudoplastic Jet." ASME-FED, 207:1-6.

Shekarriz A, JR Phillips, and TD Weir. 1995b. "Quantitative Visualization of a Submerged Pseudoplastic Jet Using Particle Image Velocimetry.” Journal of Fluids Engineering 117:369-373.

Shekarriz A, G Douillard, and CD Richards. 1996. "Velocity Measurements in a Turbulent Non-Newtonian Jet.” Journal of Fluids Engineering 118:872-874. 
Shekarriz A, KJ Hammad, and MR Powell. 1997. Evaluation of Scaling Correlations for Mobilization of Double-Shell Tank Waste. PNNL-11737, Pacific Northwest National Laboratory, Richland, Washington.

Srinarayana N, GD McBain, SW Armfield, and WX Lin. 2008. "Height and stability of laminar plane fountains in a homogeneous fluid." International Journal of Heat and Mass Transfer 51:4717-4727.

Srinarayana N, SW Armfield, and WX Lin. 2009a. "Impinging plane fountains in a homogeneous fluid." International Journal of Heat and Mass Transfer 52:2614-2623.

Srinarayana N, SW Armfield, and WX Lin. 2009b. "Laminar plane fountains impinging on a ceiling with an opposing heat flux." International Journal of Heat and Mass Transfer 52:4545-4552.

Taylor R and R Krishna. 1993. Multicomponent Mass Transfer. John Wiley \& Sons, New York.

Verhoff A. 1963. The Two-Dimensional Turbulent Wall Jet Without an External Stream. Report No. 626, Princeton University, Princeton, New Jersey.

Wiegel RL. 1964. Oceanographical Engineering. Prentice-Hall, Inc., Upper Saddle River, New Jersey, pp. 424-427.

Wells BE, CW Enderlin, Gauglitz PA, and RA Peterson. 2009. Assessment of Jet Erosion for Potential Post-Retrieval K-Basin Settled Sludge. PNNL-18831, Pacific Northwest National Laboratory, Richland, Washington.

Wells BE, PA Gauglitz, and DR Rector. 2011. Hanford Waste Physical and Rheological Properties: Data and Gaps. PNNL-20646 Rev. 0, Pacific Northwest National Laboratory, Richland, Washington.

Wells BE, PA Gauglitz, and DR Rector. 2012. Comparison of Waste Feed Delivery Small Scale Mixing Demonstration Simulant to Hanford Waste. PNNL-20637 Rev. 2, Pacific Northwest National Laboratory, Richland, Washington.

Wells BE, CW Enderlin, and MJ Minette. 2015. Technical Basis for Direct Scale-up of Small Scale Pulse Jet Mixing Results to Large Scale Vessels. PNNL-24360; WTP-RPT-240, Rev. 0, Pacific Northwest National Laboratory, Richland, Washington.

Witze PO. 1980. The impulsively Started Incompressible Turbulent Jet. SAND80-8617, Sandia National Laboratory, Sandia, New Mexico.

Yarnovsky I. 2005. "Partial Differential Equations: Graduate Level Problems and Solutions.” Available at http://www.math.ucla.edu/ yanovsky/handbooks/ODEs.pdf.

$\underline{\text { References Not Publically Available }}$

BNI. 2014. 24590-QL-HC1-M00Z-000003-04-00347, Rev. 00C, Bechtel National, Inc. Richland, Washington. 
Bontha J, C Chapman, J Geeting, and J Huckaby. 2015. "Data Summary from 8-foot Vessel Testing of Potential Pulse Jet Mixer Configurations in the Standard High Solids Vessel Design."

24590-PTF-RPT-LST-15-001, Rev 0, Bechtel National, Inc., Richland, Washington.

Braun D. 2012. 8 ft Acrylic Vessel Dimensional Inspection. TPR-LSIT-OP-0006, 24590-QL-HCI-M00Z-00003-01-00014, Rev 00A, Bechtel National, Inc., Richland, Washington.

CCN 27092, Memorandum from L. Homer (URS) to S. Anderson (URS), "Run Sheet 8' SHSVD - Tests 1-8 Rev 00,” December 8, 2014. Bechtel National, Inc., Richland Washington.

CCN272774, Meeting Minutes, "Pulse Volume Fraction - Definition and Scaling," January 22, 2015. Bechtel National, Inc., Richland, Washington.

272775, Meeting Minutes, "8-ft SHSVD Testing - Daily Tech Meetings - Week 5,” January 26-29, 2015. Bechtel National, Inc., Richland, Washington.

272778, Meeting Minutes, "8-ft SHSVD Testing - Daily Tech Meetings - Weeks 6-7," January 27 and 29 and February 2-4, 2015. Bechtel National, Inc., Richland, Washington.

272779, Meeting Minutes, "8-ft SHSVD Testing - Daily Tech Meetings - Weeks 8," February 16-20, 2015. Bechtel National, Inc., Richland, Washington.

Hazen H. 2014. Letter to RA Peterson (PNNL) from H Hazen (Bechtel National, Inc.), "Contract No. DE-AC27-01RV14136 - Hanford Tank Waste Treatment and Immobilization Plant, Memorandum of Agreement (MOA) 24590-QL-HC9-WA49-00001,- Directive Subcontract Change Notice No. 149 for WA 2014-48 FSVT Support - Increase Funding and Revised Statement of Work," November, 25, 2014, Bechtel National, Inc., Richland, Washington.

Peurrung L, D Santoski, LJ Peltier, K Recknagle, JA Bamberger, LF Pease, BE Wells, CW Enderlin, P Keuhlen, G Josephson, M Poirier, E Baer, and LA Mahoney. 2015. Technical Basis for Scaled Testing of Standard High Solids Vessel Design Mixing Systems. 24590-WTP-ES-ENG-14-017, Bechtel National, Inc., Richland, Washington. 


\section{Appendix A}

\section{Historical Breakthrough Definition and Observations}





\section{Appendix A}

\section{Historical Breakthrough Definition and Observations}

The following excerpt from LRB-BNW-14471, pp. 34-35, clarifies the historical observations and definitions associated with breakthrough in prior testing in the $12.75 \mathrm{ft}$ vessel:

Most observations made up to Dec. 4, 2003 (all observations in the large scale tank) had been with laponite. However, for the most part the same criteria apply to clay except for fracturing of the material. The observations at various conditions had been made based on observations of the liquid surface and sub surface observations through the sides of the transparent small scale tank and the submersed cameras in the large scale test setup.

Five conditions were defined leading up to break through. All five conditions were not necessarily observed prior to break through. The variation in surface conditions appears to be dependent on the initial PJM discharge velocity, the stroke length, and the time at which the PJMs had been operating. For example, if a discharge velocity $20 \mathrm{ft} / \mathrm{s}$ with a stroke of $8 \mathrm{ft}$ were to cause break through and the PJMs were started at $20 \mathrm{ft} / \mathrm{s}$ with a stroke of $8 \mathrm{ft}$ then conditions 3, 4, and 5 might be observed. However, if the PJMs were first operated at $10 \mathrm{ft} / \mathrm{s}$ for $2 \mathrm{hrs}$, followed by $15 \mathrm{ft} / \mathrm{s}$ for $4 \mathrm{hrs}$; conditions 1, 3, and 5 may be observed. The five conditions were described as:

1) Tank surface uniformly rises and falls with minimal variation in relative elevation across the un-yielded liquid surface. Visually tank surface appears homogeneous in consistency.

2) Center region of surface visually appears to be of a thinner consistency. Usually appears to be smoother and shine as though wet paint compared to the surrounding material being dried paint. Fractures may also appear in the surface. The center region may appear to rise slightly higher than the rest of the surface during the discharge portion of the PJM cycle. The center region consistency was assumed to be slow shear thinning due to the bulging/swelling created with each cycle due to the fluid plume rising up the center of the cavern.

3) Center region appears to have an elastic component as it expands due to the swell created during the discharge portion of the PJM cycle. Surface expansion is observed in which lateral flow is observed during the discharge portion of the PJM cycle but the surface appears to return to its original configuration as the surface falls during the suction phase of the PJM cycle. Several have described the center region to appear like a flower opening and closing. With each opening phase the center region of the flower appears to be of thinner material. Tracer particles or small bubbles on the surface that appear to move during the discharge portion of the cycle don't appear to experience any net movement after multiple cycles. 
4) Net lateral flow is observed in the center region, which can take on an appearance of applesauce. A combination of thinner and thicker material or perhaps many small fractures that have allowed small chunks of material to move with respect to each other. Tracer particles on the surface within the center region appear to experience net flow but they stay on the liquid surface. The surface appears to experience some surface flow but no exchange of fluid between the cavern flow and surface material appears to exist.

5) A very small upwelling of fluid is observed in the center region of the tank. The fluid in the center region of the tank appears to exchange fluid with the cavern flow.

Depending on the rate at which break through occurred, fluid from the center region may also flow out over the top of the surrounding un-yielded material. This was defined by both Carl Enderlin and Jagan Bontha as break through.

The following excerpt from LRB-BNW-14425, p.137, provides further insight into prior testing in the $12.75 \mathrm{ft}$ vessel:

During pump out of laponite from large scale tank to holding tanks located in 336 parking lot Wayne Wilcox \& I noticed several (a dozen or so) non-homogeneous chunks of laponite among the various holding tanks. These chunks were generally the size of apricots - they were blue tinted as should have been expected but had a dark center of unmixed orange dye.

These "chunks" were formed in the processing vessel even though the simulant feed had been homogeneous. Evaluation of the degree to which such "chunks" would or would not be formed in WTP vessels is outside the scope of this report. 
PNNL-24382, Rev. 1.0

WTP-RPT-239, Rev. 1.0

\section{Distribution*}

No. of

Copies

\section{INTERNAL DISTRIBUTION}

Pacific Northwest National Laboratory

JA Bamberger

JR Bontha

CW Enderlin

PA Gauglitz

JGH Geeting

KI Johnson

WL Kuhn

LA Mahoney

TE Michener

MJ Minette

LF Pease

DR Rector

BE Wells

Information Release (pdf)

Project File (1)
No. of

\section{Copies}

\section{EXTERNAL DISTRIBUTION}

Bechtel National Inc.

W Dunigon

EV Morrey

KB Peterson

LM Peurrung

WTP Docs 




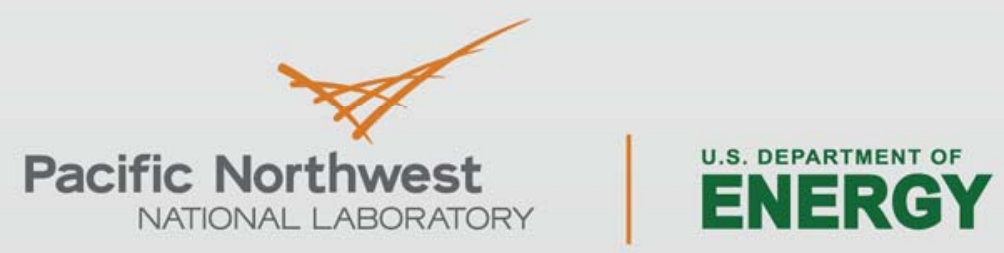

Proudly Operated by Battelle Since 1965

902 Battelle Boulevard

P.O. Box 999

Richland, WA 99352

1-888-375-PNNL (7665)

www.pnnl.gov 